\title{
MODULES OVER THE SMALL QUANTUM GROUP AND SEMI-INFINITE FLAG MANIFOLD
}

\author{
S. ARKHIPOV, R. BEZRUKAVNIKOV, A. BRAVERMAN, D. GAITSGORY, I. MIRKOVIĆ \\ To V. Drinfeld on the occasion of his 50th birthday
}

\begin{abstract}
We develop a theory of perverse sheaves on the semi-infinite flag manifold $G((t)) / N((t)) \cdot T[[t]]$, and show that the subcategory of Iwahori-monodromy perverse sheaves is equivalent to the regular block of the category of representations of the small quantum group at an even root of unity.
\end{abstract}

\section{Contents}

Introduction 2

0.1. Motivation 2

0.2. The present work 3

0.3. Contents 5

1. Background: modules over the big and small quantum groups $\quad 7$

1.1. Basics of quantum groups

1.2. $\quad$ Modules over $\mathfrak{u}_{\ell}$ as Hecke-proper modules over $\mathbf{U}_{\ell} \quad 12$

1.3. Realization via the affine Grassmannian 15

2. Some results on $\operatorname{Perv}\left(\mathrm{Gr}_{G}\right) \quad 19$

2.1. Proof of Theorem 1.3.5 19

2.2. The baby Whittaker category 22

2.3. Cosocles of costandard objects 25

3. A study of baby Verma and co-Verma modules 27

3.1. Baby co-Verma modules via $\mathbf{U}_{\ell} \quad 27$

3.2. Baby co-Verma modules via perverse sheaves on the affine Grassmannian 32

4. Sheaves on semi-infinite flags 44

4.1. Drinfeld's spaces and factorization 44

$\begin{array}{ll}\text { 4.2. A category of perverse sheaves } & 50\end{array}$

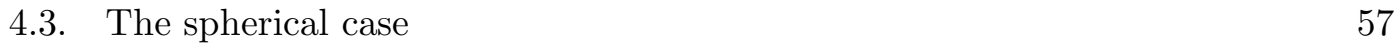

4.4. The Iwahori case 60

5. Convolution 65

5.1. Definition of convolution $\quad 65$

5.2. Exactness and smallness 68

5.3. Convolution with $\operatorname{Perv}\left(\mathrm{Gr}_{G}\right)^{I} \quad 70$

5.4. Action of convolution on standard objects 74

6. The equivalence 76

Date: April 2005. 
6.1. The functor 76

6.2. Proof of the equivalence 78

6.3. Identification of the image of baby co-Verma modules $\quad 82$

References $\quad 84$

\section{INTRODUCTION}

0.1. Motivation. Let $G$ be a reductive group. The purpose of this paper is to show that a certain remarkable abelian category $\mathcal{A}$ can be realized in (at least) three seemingly different contexts as a category of representations of some sort. This abelian category has a significance, since it can be thought of as a "local geometric Langlands" category, corresponding to an unramified local system. Let us try to explain this point, even though the local geometric Langlands correspondence has not been yet properly formulated. As a result, the discussion in this subsection will not be rigorous.

Let us recall that the global geometric Langlands correspondence aims to attach to a local system $\sigma: \pi_{1}(X) \rightarrow \breve{G}$ (here $X$ is a smooth and complete curve) a perverse sheaf $\mathcal{F}_{\sigma}$ on the stack $\operatorname{Bun}_{G}$, classifying principal $G$-bundles on $X$; one requires $\mathcal{F}_{\sigma}$ to satisfy the Hecke property with respect to $\check{G}$.

The perverse sheaf $\mathcal{F}_{\sigma}$ should be thought of as a "higher" analogue of an unramified automorphic function $f_{\sigma}$ with Langlands paramaters given by $\sigma$ (the latter makes sense, of course, only when the ground field is finite). To simplify the discussion, let us assume that the unramified automorphic representation $\pi_{\sigma}$, containing $f_{\sigma}$, lies discretely in the corresponding $L_{2}$ space and, moreover, that all of its local components are irreducible unramified principal series representations.

Let us now fix a point $x \in X$, and instead of just one automorphic function $f_{\sigma}$ let us consider the sub-space $\left(\pi_{\sigma}\right)_{x} \subset \pi_{\sigma}$, consisting of vectors invariant with respect to $\prod_{x^{\prime} \neq x} G\left(\mathcal{O}_{x^{\prime}}\right)$. This is a representation of the locally compact group $G\left(\mathcal{K}_{x}\right)$ (here for a place $x^{\prime} \in X, \mathcal{O}_{x^{\prime}}$ and $\mathcal{K}_{x^{\prime}}$ denote the local ring and the local field at this point, respectively).

According to the Langlands philosophy, $\left(\pi_{\sigma}\right)_{x}$ should be completely determined by the local Galois representation $\sigma_{x}$. Since $\sigma$ was assumed unramified, $\sigma_{x}$ boils down simply to the conjugacy class of the image of the Frobenius element.

Let us now try to guess what a geometric analogue of the vector space $\left(\pi_{\sigma}\right)_{x}$ might be. Let ${ }^{\infty} \operatorname{Bun}_{G}$ be the moduli stack of principal $G$-bundles on $X$ with a full level structure at $x$.

We propose that there should exist an (abelian) category $\mathcal{A}$, acted on by $G\left(\mathcal{K}_{x}\right)$ by functors (here $G\left(\mathcal{K}_{x}\right)$ is understood as the corresponding group ind-scheme), and a functor from $\mathcal{A}$ to the category of perverse sheaves on ${ }^{\infty} \operatorname{Bun}_{G}$, whose image consists of perverse sheaves that satisfy the Hecke property with respect to $\sigma$ an $X-x{ }^{1}$

\footnotetext{
$1_{\text {This, rather }}$ crude, form of the guess for what the local geometric Langlands correspondence might be, has been voiced independently by many people, and we by no means claim primacy in this matter.
} 
The above considerations on the function-theoretic level suggest the following candidate for $\mathcal{A}$. Namely, this should be the category of perverse sheaves on the affine Grassmannian $\operatorname{Gr}_{G}=G\left(\mathcal{K}_{x}\right) / G\left(\mathcal{O}_{x}\right)$ that satisfy the Hecke property (cf. Sect. 1.3.6 for the precise definition).

Recall now that $\left(\pi_{\sigma}\right)_{x}$ could also be realized as an (irreducible, spherical) principal series representation. Therefore, it is tempting to realize the category $\mathcal{A}$ in terms of perverse sheaves on the semi-infinite flag manifold $G\left(\mathcal{K}_{x}\right) / N\left(\mathcal{K}_{x}\right) \cdot T\left(\mathcal{O}_{x}\right)$. This is the point of departure for the present paper.

Before we proceed to the description of the concrete problem that is posed and solved here, let us mention one more incarnation of the category $\mathcal{A}$. Namely, the BeilinsonDrinfeld construction of Hecke eigensheaves via quantization of the Hitchin integrable system suggests, that the category $\mathcal{A}$ should be also equivalent to the category of modules over the affine algebra at the critical level, with a fixed central character, corresponding to some oper on the formal disc around $x$.

This category of representations can indeed be connected to $\mathcal{A}$. In the forthcoming work $[\mathrm{FG}]$ a functor is defined from the D-module version of category $\mathcal{A}$ to a certain category of modules over the affine Kac-Moody algebra at the critical level with a fixed central character. It is conjectured in $[\mathrm{FG}]$ that this functor is an equivalence of categories. Moreover, it is proved that it is fully faithful, and in the next paper the authors of loc.cit. will show that it indeed is an equivalence of categories when resricted to the Iwahori equivariant subcategories.

What is unfortunately unavailable at the moment, is a direct link between critical level representations and the cattegory of sheaves on $G\left(\mathcal{K}_{x}\right) / N\left(\mathcal{K}_{x}\right) \cdot T\left(\mathcal{O}_{x}\right)$. Such a link, which was forseen by Feigin and Frenkel in $[\mathrm{FF}]$ as a localization-type theorem for sheaves on $G\left(\mathcal{K}_{x}\right) / N\left(\mathcal{K}_{x}\right) \cdot T\left(\mathcal{O}_{x}\right)$, was the source of many people's interest in the study of both categories.

0.2. The present work. The goal of this paper is to connect the category of Hecke eigen-sheaves on the affine Grassmannian, denoted Hecke $\left(\mathrm{Gr}_{G}, \check{G}\right.$ ) (or rather its graded version, denoted $\dot{H}$ ecke $\left.\left(\mathrm{Gr}_{G}, \check{G}\right)\right)$, to the category of perverse sheaves on the semi-infinite flag manifold. An immediate problem that one runs into is that the latter category does not a priori makes sense:

The semi-infinite flag manifold, thought of as $G\left(\mathcal{K}_{x}\right) / N\left(\mathcal{K}_{x}\right) \cdot T\left(\mathcal{O}_{x}\right)$, does not carry an algebro-geometric structure that would allow for the theory of perverse sheaves, or D-modules, in the way it is known today.

We get around this difficulty as follows. We define an "artificial" category $\operatorname{Perv}\left(\mathcal{F} l^{\frac{\infty}{2}}\right)$ that possesses the natural properties that one expects from the yet non-existing category of perverse sheaves on $G\left(\mathcal{K}_{x}\right) / N\left(\mathcal{K}_{x}\right) \cdot T\left(\mathcal{O}_{x}\right)$. The approach to the definition of $\operatorname{Perv}\left(\mathcal{F} l^{\frac{\infty}{2}}\right)$, developed in this paper, was initiated in $[\mathrm{FM}]$, and it uses a geometric object, denoted $\overline{\mathrm{Bun}}_{N^{-}}$, introduced by Drinfled.

The space $\overline{\mathrm{Bun}}_{N^{-}}$is a finite-dimensional (or, rather, ind-finite dimensional) approximation to $G\left(\mathcal{K}_{x}\right) / N\left(\mathcal{K}_{x}\right) \cdot T\left(\mathcal{O}_{x}\right)$, and it has as an input a global curve $X$. By definition, $\overline{\mathrm{Bun}}_{N^{-}}$classifies principal $G$-bundles on $X$ endowed with a possibly degenerate 
reduction to the maximal unipotent subgroup $N^{-}$, and it contains the stack $\operatorname{Bun}_{N^{-}}$ classifying $N^{-}$-bundles on $X$ as an open substack.

The realization of $\operatorname{Perv}\left(\mathcal{F} l^{\frac{\infty}{2}}\right)$ via $\overline{\mathrm{Bun}}_{N^{-}}$is natural from the geometric Langlands perspective as well: the space $\overline{\mathrm{Bun}}_{N^{-}}$is used to define geometric Eisenstein series by taking the direct image under the natural projection to $\operatorname{Bun}_{G}$ (cf. [BG]). Therefore, such incarnation of $\operatorname{Perv}\left(\mathcal{F} l^{\frac{\infty}{2}}\right)$ implies the existence of a functor from $\mathcal{A}$ to the (derived) category of perverse sheaves on ${ }^{\infty} \operatorname{Bun}_{G}$.

Having defined the category $\operatorname{Perv}\left(\mathcal{F} l^{\frac{\infty}{2}}\right)$, we have at our disposal a naturally defined functor from $\dot{H}$ ecke $\left(\mathrm{Gr}_{G}, \check{G}\right)$ to it. However, we do not have any real evidence as to whether this functor should be an equivalence. Quite possibly, to make this functor an equivalence, one has to modify both categories by imposing some Noetherianness condions on the $\dot{H}$ ecke $\left(\mathrm{Gr}_{G}, \check{G}\right)$ side, and restrictions on the behaviour "at the boundary" on the $\operatorname{Perv}\left(\mathcal{F} l^{\frac{\infty}{2}}\right)$ side.

The problem arising here is similar to the one in the definition of the Schwarz space on $G\left(\mathcal{K}_{x}\right) / N\left(\mathcal{K}_{x}\right)$ in the function-theoretic context in [BK]. Identifying the image of Hecke $\left(\mathrm{Gr}_{G}, \check{G}\right)$ inside Perv $\left(\mathcal{F l} l^{\frac{\infty}{2}}\right)$ appears to be an interesting problem, and it is closely related to giving a geometric definition of Fourier-transform functors of loc. cit.

However, if instead of the entire $\dot{H}$ ecke $\left(\operatorname{Gr}_{G}, \check{G}\right)$ and $\operatorname{Perv}\left(\mathcal{F} l^{\frac{\infty}{2}}\right)$ we work with the subcategories, denoted $\dot{H}$ ecke $\left(\operatorname{Gr}_{G}, \check{G}\right)^{I^{0}}$ and $\operatorname{Perv}\left(\mathcal{F} l^{\frac{\infty}{2}}\right)^{I^{0}}$, respectively, consisting of Iwahori-monodromic objects, the required Noetherian and boundary conditions are easy to spell out, simply by requiring that our objects have finite length.

Thus, the main result of this paper, Theorem 6.1.6, states that the category, denoted $\dot{H}$ ecke $\left(\mathrm{Gr}_{G}, \check{G}\right)_{A r t}^{I^{0}}$, consisting of Artinian and Iwahori-monodromic objects in $\dot{\mathrm{H}} \mathrm{ecke}\left(\mathrm{Gr}_{G}, \check{G}\right)$, is equivalent to the subcategory of Artinian objects in $\operatorname{Perv}\left(\mathcal{F} l^{\frac{\infty}{2}}\right)^{I^{0}}$.

The method of proof of Theorem 6.1.6 relies rather heavily on the specifics of Iwahorimonodromic situation. Namely, we use the fact that both categories are hereditary (i.e., in many ways similar to the usual category $\mathcal{O}$ ). In particular, they both have standard and costandard objects, numbered by elements of the extended affine Weyl group $W_{\text {aff }}$, etc.

The hereditary structure on $\operatorname{Perv}\left(\mathcal{F} l^{\frac{\infty}{2}}\right)^{I^{0}}$ is evident basically from the stratification of $G\left(\mathcal{K}_{x}\right) / N\left(\mathcal{K}_{x}\right) \cdot T\left(\mathcal{O}_{x}\right)$ by Iwahori orbits. However, for $\dot{\bullet}$ ecke $\left(\mathrm{Gr}_{G}, \check{G}\right)_{\text {Art }}^{I^{0}}$ this structure is not so evident, and it comes from another crucial ingredient of this paper, namely, the equivalence between $\dot{H}$ ecke $\left(\mathrm{Gr}_{G}, \check{G}\right)_{\text {Art }}^{I^{0}}$ and the regular block of the category of representations of the small quantum group, corresponding to $G$, at an even root of unity.

The latter equivalence results by combining the main result of $[\mathrm{ABG}]$ that links representations of the big quantum group and perverse sheaves on $\mathrm{Gr}_{G}$, and [AG], where an explicit relation between the categories of representations of the big and small quantum group is established. 
We should point out, however, that the present paper relies formally on neither $[\mathrm{ABG}]$, nor $[\mathrm{AG}]$. We supply purely geometric proofs for all the statements needed to establish the hereditary property of $\dot{\operatorname{Hecke}}\left(\mathrm{Gr}_{G}, \breve{G}\right)_{A r t}^{I^{0}}$. But these statements would be rather hard to guess, had we not had the equivalence with the quantum group as a guide.

As a result, we also obtain that the category of Artinian objects in $\operatorname{Perv}\left(\mathcal{F} l^{\frac{\infty}{2}}\right) I^{0}$ is equivalent to the category $\dot{\mathfrak{u}}_{\ell}-\bmod _{0}$-the above mentioned regular block in the category of $\mathfrak{u}_{\ell}$-modules. This is our Theorem 6.1.7, which concludes the project of proving such an equivalence, initiated and advanced almost to the end by M. Finkelberg. ${ }^{2}$

0.3. Contents. Let us now discuss the organization and contents of the present paper.

Section 1 reviews the theory of modules over the big and small quantum groups. In Sect. 1.1 we recall the basic definitions related to corresponding categories of representations, and the quantum Frobenius homomorphism. In Sect. 1.2 we recall the realization of the category of representations of the small quantum group as representations of the big quantum group, satisfying the Hecke property. In Sect. 1.3 we recall the $[\mathrm{ABG}]$ equivalence between the regular block of the category of representations of the big quantum group and Iwahori-monodromic perverse sheaves on the affine Grassmannian; we also introduce the category of Hecke eigen-sheaves on the Grassmannian and discuss its relation to the category of representations of the small quantum group.

Section 2 reviews some basic properties of Iwahori-equivariant perverse sheaves on the affine Grassmannian. In Sect. 2.1 we give a geometric proof of an irreducibility result on convolution of certain perverse sheaves, which translates by means of [ABG] to the Steinberg-type theorem for representations of the quantum group; some ingredients of the proof will be used later on for a crucial irreducibility result in Sect. 5.3. In Sect. 2.2 we discuss the baby Whittaker category on the affine Grassmannian and its relation to a certain Serre quotient category of $\operatorname{Perv}\left(\mathrm{Gr}_{G}\right)^{I^{0}}$; the discussion here largely repeats the one in $[\mathrm{AB}]$. In Sect. 2.3 we apply the results of the previous subsection to establish a crucial result about cosocles of some costandard objects in $\operatorname{Perv}\left(\operatorname{Gr}_{G}\right)^{I^{0}}$; this result will be essential for the proof of the main theorem.

Section 3 is devoted to the study of baby (co)Verma modules over the small quantum group, which are the building blocks of the category of its representations. In Sect. 3.1 we translate the properties of baby co-Verma modules into properties of the corresponding modules over the big quantum group, satisfying the Hecke property. In Sect. 3.2 we reprove the corresponding facts (often by different methods) in the context of Iwahori-monodromic perverse sheaves on $\mathrm{Gr}_{G}$.

In Section 4 we discuss the main object of study of this paper, namely, the category $\operatorname{Perv}\left(\mathcal{F} l^{\frac{\infty}{2}}\right)$, which is a surrogate for the non-existing category of perverse sheaves on $G\left(\mathcal{K}_{x}\right) / N\left(\mathcal{K}_{x}\right) \cdot T\left(\mathcal{O}_{x}\right)$.

\footnotetext{
${ }^{2}$ An equivalence between $\dot{\mathfrak{u}}_{\ell}-\bmod _{0}$ and the would-be category of Iwahori-monodromic perverse sheaves on $G\left(\mathcal{K}_{x}\right) / N\left(\mathcal{K}_{x}\right) \cdot T\left(\mathcal{O}_{x}\right)$ has also been guessed independently by several people, among them, Lusztig and Feigin-Frenkel, but we could not find a precisely formulated conjecture in the literature. Our formulation as well as the strategy of the proof are due to Finkelberg.
} 
In Sect. 4.1 we discuss the underlying geometric object - the stack $\overline{\mathrm{Bun}}_{N^{-}}$along with its numerous variants. In Sect. 4.2 we finally introduce the category $\operatorname{Perv}\left(\mathcal{F} l^{\frac{\infty}{2}}\right)$, the main technical ingredient being the factorizability property, observed in [FFKM]; we show that that $\operatorname{Perv}\left(\mathcal{F} l^{\frac{\infty}{2}}\right)$ by and large behaves in the way one expects from the analogy with $G\left(\mathcal{K}_{x}\right) / N\left(\mathcal{K}_{x}\right) \cdot T\left(\mathcal{O}_{x}\right)$. In Sect. 4.3 we study the most basic objects in $\operatorname{Perv}\left(\mathcal{F} l^{\frac{\infty}{2}}\right)$, namely, the spherical ones, and show that the resulting category is semi-simple. Finally, in Sect. 4.4 we discuss the Iwahori-monodromic subcategory of $\operatorname{Perv}\left(\mathcal{F} l^{\frac{\infty}{2}}\right)$, and prove some results that are parallel to the corresponding assertions about Iwahori-monodromic sheaves on $\mathrm{Gr}_{G}$.

As was mentioned above, the category $\operatorname{Perv}\left(\mathcal{F} l^{\frac{\infty}{2}}\right)$ must be acted on by the group ind-scheme $G\left(\mathcal{K}_{x}\right)$ by auto-functors. A rigorous incarnation of this phenomenon is the action of perverse sheaves on $G\left(\mathcal{K}_{x}\right)$ by Hecke functors (the latter are defined on the level of the corresponding derived category). In Sect. 5 we study this convolution action in our realization of $\operatorname{Perv}\left(\mathcal{F l} l^{\frac{\infty}{2}}\right)$ via $\overline{\operatorname{Bun}}_{N^{-}}$.

In Sect. 5.1 we define the convolution action and show that it indeed respects the category $\operatorname{Perv}\left(\mathcal{F} l^{\frac{\infty}{2}}\right)$. In Sect. 5.2 we establish a crucial semi-smallness result that allows to pass from perverse sheaves on $\operatorname{Gr}_{G}$ to $\operatorname{Perv}\left(\mathcal{F} l^{\frac{\infty}{2}}\right)$ (this is largely borrowed from $[\mathrm{FM}]$ and $[\mathrm{BG}])$. In Sect. 5.3 we refine the discussion of the previous subsection and show that certain convolution diagrams give rise to small (vs. semi-small) maps, thereby implying certain irreducibility properties. In Sect. 5.4 we establish another important technical result that describes the convolution of standard objects.

Finally, in Section 6 we state and prove the equivalence between the subcategories of Artinian objects in $\stackrel{\operatorname{Hecke}}{ }\left(\mathrm{Gr}_{G}, \check{G}\right)^{I^{0}}$ and $\operatorname{Perv}\left(\mathcal{F} l^{\frac{\infty}{2}}\right)^{I^{0}}$. In Sect. 6.1 we define the required functor. In Sect. 6.2 we show that this functor is exact and reduce the equivalence assertion to a computation of the image of baby co-Verma modules. In Sect. 6.3 we perform the required calculation using some information on cosocles of costandard objects in both categories.

The conventions adopted in this paper regarding the quantum group follow those of $[\mathrm{AG}]$. Conventions and notation concerning the affine Grasmannian and Drinfeld's compactifications follow those of $[\mathrm{BG}]$. To fix the context we will work with varieties and stacks over the ground field $\mathbb{C}$, and holonomic D-modules (but we will still call them perverse sheaves). If $y$ is a smooth variety, $\underline{\mathbb{C}}_{y}$ will denote the (cohomologically shifted) D-module, corresponding to the constant sheaf on it.

Acknowledgements. As was mentioned earlier, the problem solved in this paper was both posed (and the method of solution was suggested) by M. Finkelberg back in 1998, when the authors were at IAS, Princeton, for the special year on geometric representation theory. We are grateful to him for the permission to publish many of his results and ideas.

We would also like to thank A. Beilinson, V. Drinfeld, B. Feigin, E. Frenkel and D. Kazhdan for sharing their ideas and stimulating discussions. 
It is an honour for us to dedicate this paper to Vladimir Drinfeld. Along with numerous other things in modern mathematics, the three main objects of study in this paper-quantum groups, Hecke eigen-sheaves and $\overline{\mathrm{Bun}}_{N^{-}}$were invented by him.

\section{BACKGRound: MOdules OVER THE BIG AND SMALl QUANTUM GROUPS}

\subsection{Basics of quantum groups.}

1.1.1. Root data. Let $G$ be a reductive group with connected center. Let $\breve{G}$ be its Langlands dual; by assumption the derived group of $\check{G}$ is simply connected. ${ }^{3}$

We will denote by $T$ (resp., $\check{T}$ ) the Cartan group of $G$ (resp., $\breve{G}$ ), and by $W$ the Weyl group. We fix Borel subgroups $B, B^{-} \subset G$ (resp., $\check{B}, \check{B}^{-} \subset \check{G}$ ) and think of $T$ (resp., $\check{T}$ ) as a subgroup of $G$ (resp., $\check{G}$ ) equal to their intersection.

We will denote by $\check{\Lambda}$ (resp., $\Lambda$ ) the coweight (resp., weight lattice) of $G$; by $\check{\Lambda}^{+}$(resp., $\Lambda^{+}$) we will denote the subset of dominant coweights (resp., weights). We will denote by $\langle\cdot, \cdot$,$\rangle the pairing between the two. We will denote by W_{\text {aff }}$ the extended Weyl group $W \ltimes \check{\Lambda}$.

Let $\mathcal{J}$ be the set of vertices of the Dynkin graph of $G$; for $\imath \in \mathcal{J}$ we will denote by $\check{\alpha}_{\imath} \in \check{\Lambda}$ (resp., $\alpha_{\imath} \in \Lambda$ ) the corresponding simple coroot (resp., root). We will denote by $\check{\Lambda}^{\text {pos }}$ (resp., $\Lambda^{\text {pos }}$ ) the sub-semigroup spanned by positive coroots (resp., roots).

Let $(\cdot, \cdot): \operatorname{Span}\left\{\alpha_{\imath}\right\} \otimes \operatorname{Span}\left\{\alpha_{\imath}\right\} \rightarrow \mathbb{Z}$ be the canonical inner form. In other words, $\left\|\alpha_{\imath}\right\|^{2}=2 d_{\imath}$, where $d_{\imath} \in\{1,2,3\}$ is the minimal set of integers such that the matrix $\left(\alpha_{\imath}, \alpha_{\jmath}\right):=d_{\imath} \cdot\left\langle\alpha_{\imath}, \check{\alpha}_{\jmath}\right\rangle$ is symmetric.

We choose a symmetric $W$-invariant form $(\cdot, \cdot)_{\ell}: \check{\Lambda} \otimes \check{\Lambda} \rightarrow \mathbb{Z}$, such that there exists a sufficiently large positive even integer $\ell$, divisible by all $d_{\imath}$, such that

$$
\left(\check{\alpha}_{\imath}, \check{\lambda}\right)_{\ell}=\ell_{\imath} \cdot\left\langle\alpha_{\imath}, \check{\lambda}\right\rangle
$$

$\forall \check{\lambda} \in \check{\Lambda}$, where $\ell_{\imath}=\frac{\ell}{d_{2}}$.

We will denote by $\phi_{\ell}$ the resulting map $\check{\Lambda} \rightarrow \Lambda$, and also the map $T \rightarrow \check{T}$.

1.1.2. The big quantum group. As was mentioned earlier, our conventions regarding representations of the big quantum group follow those of $[\mathrm{AG}]$. Let $\mathbf{U}_{\ell}$-mod be the category of representations of the big quantum group, corresponding to $G$ and $\ell$. By definition, objects of this category are finite-dimensional vector spaces, acted on by the algebraic group $T$, and the operators $E_{\imath}, F_{\imath}, E_{\imath}^{\left(\ell_{2}\right)}, F_{\imath}^{\left(\ell_{2}\right)}$, that satisfy the well-known relations. The category $\mathbf{U}_{\ell}$-mod has a natural monoidal structure.

We will denote by $\mathbf{U}_{\ell}-\overline{\bmod }$ the ind-completion of $\mathbf{U}_{\ell}$-mod. I.e., this is the category of infinite-dimensional vector spaces, acted on by the same set of operators, which can be represented as unions of finite-dimensional sub-representations.

Let $\mathbf{B}_{\ell}^{-}$-mod be the category of representations of the "negative quantum Borel". I.e., objects of this category are finite-dimensional vector spaces, acted on by the algebraic group $T$, and the operators $F_{\imath}, F_{\imath}^{\left(\ell_{l}\right)}$, which satisfy the same relations. This

\footnotetext{
${ }^{3}$ For what follows we could replace $G$ by an isogenous group such that $[G, G]$ is simply connected. In this case $\check{G}$ also has connected center.
} 
is also a monoidal category and there exists a natural forgetful monoidal functor $\operatorname{Res}_{\mathbf{B}_{\ell}^{-}}^{\mathbf{U}_{\ell}}: \mathbf{U}_{\ell}-\bmod \rightarrow \mathbf{B}_{\ell}^{-}$-mod. This functor admits a right adjoint, denoted by $\operatorname{Ind}_{\mathbf{B}_{\ell}^{-}}^{\mathbf{U}_{\ell}}$.

In addition there exists a natural functor $\operatorname{Rep}(T) \rightarrow \mathbf{B}_{\ell}^{-}$-mod, where we let the operators $F_{\imath}, F_{\imath}^{\left(\ell_{2}\right)}$ act trivially on the corresponding vector space.

For $\lambda \in \Lambda$ we let $\mathbf{W}^{\lambda} \in \mathbf{U}_{\ell}$-mod be the dual Weyl module defined as $\left.\operatorname{Ind}_{\mathbf{B}_{\ell}^{-}} \mathbf{U}^{\lambda}\right)$, where $\mathbf{C}^{\lambda}$ is the 1-dimensional representation of $T$, corresponding to $\lambda$. It is known that $\mathbf{W}^{\lambda} \neq 0$ if and only if $\lambda \in \Lambda^{+}$. It is also known that $\mathbf{W}^{\lambda}$ admits a unique irreducible submodule, denoted $\mathbf{L}^{\lambda}$, and this establishes a bijection between $\Lambda^{+}$and the set of irreducibles in $\mathbf{U}_{\ell}$-mod.

As every Artinian category, $\mathbf{U}_{\ell}$-mod splits into a direct sum of indecomposable Artinian categories, called blocks. Slightly deviating from the accepted conventions, we will denote by $\mathbf{U}_{\ell}-\bmod _{0}$ the direct summand of $\mathbf{U}_{\ell}$-mod that contains the irreducibles $\mathbf{L}^{\lambda}$ for $\lambda$ of the form

$$
w(\rho)-\rho+\phi_{\ell}(\check{\lambda})
$$

$w \in W, \check{\lambda} \in \check{\Lambda}$.

We will denote by $\mathbf{U}_{\ell}-\overline{\bmod }_{0}$ the ind-completion of $\mathbf{U}_{\ell}-\bmod _{0}$, which is a direct summand in $\mathbf{U}_{\ell}-\overline{\bmod }$.

1.1.3. Quantum Frobenius homomorphism. Let $\operatorname{Rep}(\check{G})$ denote the category of finitedimensional representations of $\breve{G}$. Following [Lu1] there exists a monoidal functor

$$
\text { Fr : } \operatorname{Rep}(\check{G}) \rightarrow \mathbf{U}_{\ell}-\bmod ,
$$

defined as follows. For $V \in \operatorname{Rep}(\check{G})$, the representation $\operatorname{Fr}(V)$ occurs on the same underlying vector space, denoted $\underline{V}$, and the action of $T$ is given via $\phi_{\ell}: T \rightarrow \check{T}$. The operators $E_{\imath}, F_{\imath}$ act trivially, and $E_{\imath}^{\left(\ell_{\imath}\right)}, F_{\imath}^{\left(\ell_{\imath}\right)}$ act via the Chevalley generators $e_{\imath}, f_{\imath} \in \check{\mathfrak{g}}$.

It is known that the functor Fr is fully faithful. Moreover, for $\check{\lambda} \in \check{\Lambda}^{+}$

$$
\operatorname{Fr}\left(V^{\check{\lambda}}\right) \simeq \mathbf{L}^{\phi_{\ell}(\check{\lambda})}
$$

where $V^{\check{\lambda}}$ denotes the corresponding irreducible representation of $\check{G}$.

Let recall that a dominant weight $\lambda$ is called restricted if $\forall \imath \in \mathcal{J}$

$$
\left\langle\lambda, \check{\alpha}_{\imath}\right\rangle<\ell_{\imath} \text {. }
$$

We have the following fundamental result:

Theorem 1.1.4. If $\check{\lambda} \in \Lambda^{+}$is restricted, then for every $\check{\mu} \in \check{\Lambda}^{+}$

$$
\operatorname{Fr}\left(V^{\check{\mu}}\right) \otimes \mathbf{L}^{\lambda} \simeq \mathbf{L}^{\lambda+\phi_{\ell}(\check{\mu})} .
$$

Since every $\lambda \in \Lambda^{+}$can be written as $\check{\lambda}_{1}+\check{\lambda}_{2}$ with $\check{\lambda}_{1}$ restricted and $\check{\lambda}_{2}$ in the image of $\check{\Lambda}^{+}$, the above theorem describes all irreducibles in $\mathbf{U}_{\ell}$-mod. (Note that the decomposition of a weight as above is unique modulo elements $\nu \in \Lambda$, orthogonal to all roots, i.e., those for which $\mathbf{L}^{\nu}$ is 1-dimensional.)

Corollary 1.1.5. The functor $M \mapsto \operatorname{Fr}(V) \otimes M: \mathbf{U}_{\ell}$-mod $\rightarrow \mathbf{U}_{\ell}$-mod preserves $\mathbf{U}_{\ell}-\bmod _{0}$. 
MODULES OVER THE SMALL QUANTUM GROUP AND SEMI-INFINITE FLAG MANIFOLD 9

1.1.6. The graded small quantum group. We define the category of representations of the graded small quantum group $\dot{\mathfrak{u}}_{\ell}$-mod to consist of finite-dimensional vector spaces, acted on by the algebraic group $T$ and the operators $E_{\imath}, F_{\imath}$, satisfying the usual relations. This is also a monoidal category, and we have a monoidal forgetful functor $\operatorname{Res}_{\mathfrak{u}_{\ell}}^{\mathbf{U}_{\ell}}: \mathbf{U}_{\ell}-\bmod \rightarrow \dot{\mathfrak{u}}_{\ell}-\bmod$.

In addition, we have a fully-faithful functor $\operatorname{Rep}(\check{T}) \rightarrow \dot{\mathfrak{u}}_{\ell}$-mod. By a slight abuse of notation we will denote by $\mathbf{C}^{\check{\mu}}$ the 1 -dimensional module over $\mathfrak{\mathfrak { u }}_{\ell}$, corresponding to $\check{\mu} \in \check{\Lambda}$.

Let $\mathfrak{b}_{\ell}^{-}$-mod be the category of representations of the corresponding "graded small negative Borel subgroup". I.e., this is the category of vector spaces, acted on by $T$ and the $F_{\imath}$ 's, satisfying the same relations. We will denote by $\operatorname{Res}_{\dot{b}_{\ell}^{-}}^{\mathfrak{\mathfrak { i }}_{\ell}}$ the forgetful functor

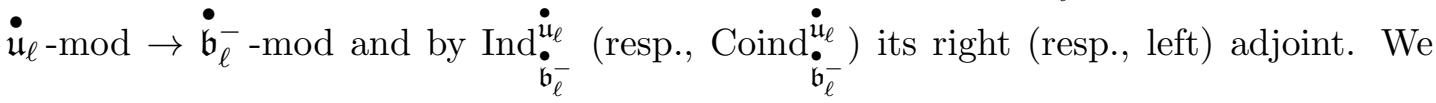
also have a functor $\operatorname{Rep}(T) \rightarrow \dot{\mathfrak{b}}_{\ell}^{-}$-mod.

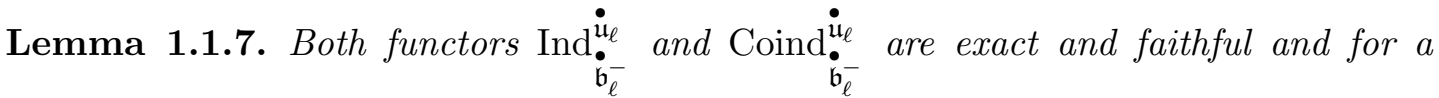
character $\lambda$ of $T$,

$$
\operatorname{Ind}_{\mathfrak{b}_{\ell}^{-}} \underset{\mathfrak{u}_{\ell}}{\mathfrak{u}_{\ell}}\left(\mathbf{C}^{\lambda}\right) \simeq \underset{\mathfrak{b}_{\ell}^{-}}{\operatorname{Coind}^{\mathfrak{u}_{\ell}}}\left(\mathbf{C}^{\lambda-\phi_{\ell}(2 \check{\rho})+2 \rho}\right) .
$$

We will denote the module $\operatorname{Ind}_{\dot{\mathfrak{b}}_{\ell}^{-}}^{\mathfrak{\mathfrak { u }}_{\ell}}\left(\mathbf{C}^{\lambda}\right)$ by $\dot{M}^{\lambda}$ and call it the baby co-Verma module of highest weight $\lambda$. One easily shows that the socle of each $\dot{M}^{\lambda}$ is simple. We will denote the corresponding irreducible by $\dot{L}^{\lambda}$. Thus we obtain a bijection between $\Lambda$ and the set of irreducibles in $\mathfrak{u}_{\ell}$-mod.

For $\check{\mu} \in \check{\Lambda}$, we have:

$$
\dot{M}^{\lambda+\phi_{\ell}(\check{\mu})} \simeq \mathbf{C}^{\check{\mu}} \otimes \dot{M}^{\lambda} \text { and } \dot{L}^{\lambda+\phi_{\ell}(\check{\mu})} \simeq \mathbf{C}^{\check{\mu}} \otimes \dot{L}^{\lambda} .
$$

In addition, we have the following result:

Proposition 1.1.8. If $\lambda$ is dominant and restricted,

$$
\dot{L}^{\lambda} \simeq \operatorname{Res}_{\mathfrak{u}_{\ell}}^{\mathbf{U}_{\ell}}\left(\mathbf{L}^{\lambda}\right)
$$

Being Artinian, the category $\mathfrak{\mathfrak { u }}_{\ell}$-mod also admits a decomposition into blocks. We will denote by $\dot{\mathfrak{u}}_{\ell}-\bmod _{0}$ the direct summand of $\mathfrak{\mathfrak { u }}_{\ell}$-mod that contains the irreducibles $\dot{L}^{\lambda}$ for $\lambda$ of the form $w(\rho)-\rho+\phi_{\ell}(\check{\lambda}) w \in W, \check{\lambda} \in \check{\Lambda}$.

Lemma 1.1.9. The sub-category $\mathbf{U}_{\ell}-\bmod _{0} \subset \mathbf{U}_{\ell}$-mod is the preimage of $\dot{u}_{\ell}-\bmod _{0} \subset$ $\dot{\mathfrak{u}}_{\ell}-\bmod _{0}$ under the forgetful functor $\operatorname{Res}_{\mathfrak{u}_{\ell}}^{\mathbf{U}_{\ell}}$, 
Finally, we will denote by $\dot{\mathfrak{u}}_{\ell}-\overline{\bmod }\left(\right.$ resp., $\left.\mathfrak{\mathfrak { u }}_{\ell}-\overline{\bmod }_{0}\right)$ the ind-completion of $\dot{\mathfrak{u}}_{\ell}$-mod (resp., $\left.\dot{\mathfrak{u}}_{\ell}-\bmod _{0}\right)$.

1.1.10. The non-graded small quantum group. We define the category $\mathfrak{u}_{\ell}$-mod to consist of finite-dimensional vector spaces, acted on by the group $T_{\ell}:=\operatorname{ker}\left(\phi_{\ell}: T \rightarrow \check{T}\right)$, and the operators $K_{\imath} \cdot E_{\imath}, F_{\imath}$, subject to the usual relations. Note that $\mathfrak{u}_{\ell}$-mod is not a monoidal category; however, we have a well-defined functor of tensor product on the right by an object of $\mathfrak{\mathfrak { u }}_{\ell}$-mod:

$$
N \in \mathfrak{u}_{\ell}-\bmod , M \in \dot{\mathfrak{u}}_{\ell}-\bmod \mapsto N \otimes \operatorname{Res}_{\mathfrak{u}_{\ell}} \dot{\mathfrak{u}}_{\ell}(M) .
$$

The following proposition describes the relation between the small quantum group and the quantum Frobenius homomorphism:

\section{Proposition 1.1.11.}

(1) For $M \in \dot{\mathfrak{u}}_{\ell}-\bmod$ and $\check{\lambda} \in \check{\Lambda}$,

$$
\operatorname{Res}_{\mathfrak{u}_{\ell}}^{\mathfrak{\mathfrak { u }}_{\ell}}\left(\mathbf{C}^{\check{\lambda}} \otimes M\right) \simeq \operatorname{Res}_{\mathfrak{u}_{\ell}}^{\mathfrak{\mathfrak { u }}_{\ell}}(M)
$$

(2) For $M$ as above the maximal trivial sub- (resp., quotient-) object $N^{\prime}$ of $\operatorname{Res}_{\mathfrak{u}_{\ell}}^{\dot{\mathfrak{u}}_{\ell}}(M)$ comes from a sub-(resp., quotient-) object $M^{\prime}$ of $M$, which is in the image of the functor $\operatorname{Rep}(\check{T}) \rightarrow \dot{\mathfrak{u}}_{\ell}$-mod.

(3) For $M \in \mathbf{U}_{\ell}$-mod, $V \in \operatorname{Rep}(\check{G})$,

$$
\operatorname{Res}_{\mathfrak{u}_{\ell}}^{\mathbf{U}_{\ell}}(\operatorname{Fr}(V) \otimes M) \simeq \underset{\check{\nu}}{\oplus} \mathbf{C}^{\check{\nu}} \otimes \operatorname{Res}_{\mathfrak{u}_{\ell}}^{\mathbf{U}_{\ell}}(M) \otimes \underline{V}(\check{\nu}),
$$

where $\underline{V}(\check{\nu})$ denotes the $\check{\nu}$-weight space of $V$, and $\mathbf{C}^{\check{\nu}}$ the corresponding 1-dimensional representation of $\mathfrak{\mathfrak { u }}_{\ell}$.

(4) For an object $M \in \mathbf{U}_{\ell}$-mod the maximal trivial sub- (resp., quotient-) object $N^{\prime}$ of $\operatorname{Res}_{\mathfrak{u}_{\ell}}^{\mathbf{U}_{\ell}}(M)$, comes from a sub- (resp., quotient-) object $M^{\prime}$ of $M$, which is in the image of the functor Fr.

Let $\mathfrak{b}_{\ell}^{-}$-mod be the category consisting of finite-dimensional vector spaces, acted on by the group $T_{\ell}$ and the operators $F_{\imath}$, satisfying the usual relations. We have the evident functor $\operatorname{Rep}\left(T_{\ell}\right) \rightarrow \mathfrak{b}_{\ell}^{-}$-mod, such that the analog of Lemma 1.1.7 holds. For a character $\bar{\lambda}: T_{\ell} \rightarrow \mathbb{C}^{*}$ we will denote by $M^{\bar{\lambda}}$ the module $\operatorname{Ind}_{\mathfrak{b}_{\ell}^{-}}^{\mathfrak{u}_{\ell}}\left(\mathbb{C}^{\bar{\lambda}}\right)$.

We have:

Lemma 1.1.12. For a character $\lambda \in \Lambda$ we have:

(1) $\operatorname{Res}_{\mathfrak{u}_{\ell}}^{\dot{\mathfrak{u}}_{\ell}}\left(\dot{M}^{\lambda}\right) \simeq M^{\bar{\lambda}}$, where $\bar{\lambda}$ is the restriction of $\lambda$ to $T_{\ell}$.

(2) The module $L^{\bar{\lambda}}:=\operatorname{Res}_{\mathfrak{u}_{\ell}} \dot{\mathfrak{u}}_{\ell}\left(\dot{L}^{\lambda}\right)$ depends only on the class of $\lambda$ modulo $\phi_{\ell}(\check{\Lambda})$, and is irreducible. Moreover, these are all the irreducibles in $\mathfrak{u}_{\ell}$-mod.

Let $\mathfrak{u}_{\ell}-\bmod _{0}$ be the direct summand of $\mathfrak{u}_{\ell}$-mod, that contains the trivial representation. 
Lemma 1.1.13. The subcategory $\dot{\mathfrak{u}}_{\ell}-\bmod _{0} \subset \mathfrak{\mathfrak { u }}_{\ell}-\bmod$ is the preimage of $\mathfrak{u}_{\ell}-\bmod _{0} \subset$ $\mathfrak{u}_{\ell}$-mod under the forgetful functor.

We will denote by $\mathfrak{u}_{\ell}-\overline{\bmod }\left(\right.$ resp., $\left.\mathfrak{u}_{\ell}-\overline{\bmod }_{0}\right)$ the ind-completion of $\mathfrak{u}_{\ell}$-mod (resp., $\left.\mathfrak{u}_{\ell}-\bmod _{0}\right)$.

In the sequel we will need the following assertion:

Proposition 1.1.14. There exists a fully-faithful functor $\operatorname{Fr}_{B^{-}}: \operatorname{Rep}\left(\check{B}^{-}\right) \rightarrow \mathbf{B}_{\ell}^{-}$-mod, such that

(1) We have a commutative diagram of functors.

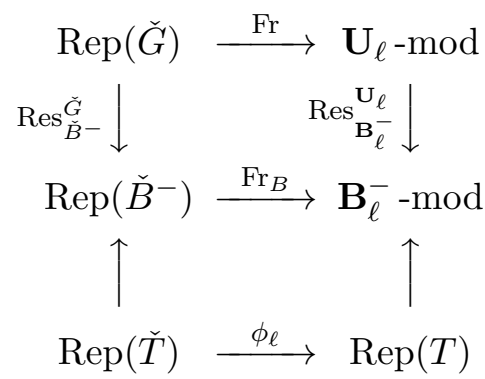

(2) For $N \in \mathbf{B}_{\ell}^{-}$-mod the maximal sub-(resp., quotient-) space of $N$, on which $\mathfrak{b}_{\ell}^{-}$acts trivially, is a sub-(resp., quotient-) module, which lies in the image of the functor $\operatorname{Fr}_{B}$.

1.1.15. Weyl group action. Following Lusztig, to every element $w$ of the Weyl group we can attach an invertible operator acting functorially on the vector space underlying every object of $\mathbf{U}_{\ell}$-mod, or which is the same, an automorphism of the forgetful functor $\mathbf{U}_{\ell}$-mod $\rightarrow$ Vect. This automorphism is well-defined modulo elements of $T$.

This construction can be reformulated as follows. To every $w \in W$ we attach a selffunctor $\mathrm{F}_{w}: \mathbf{U}_{\ell}$-mod $\rightarrow \mathbf{U}_{\ell}$-mod, that commutes with the forgetful functor to vector spaces, and an isomorphism

$$
w_{\ell}: \operatorname{Id}_{\mathbf{U}_{\ell}-\bmod } \Rightarrow \mathrm{F}_{w} .
$$

Restricting these data to the sub-category $\operatorname{Rep}(\check{G}) \subset \mathbf{U}_{\ell}$-mod we obtain that the pair $\left(\mathrm{F}_{w}, w_{\ell}\right)$ gives rise to an element $w_{\breve{G}} \in \check{G}$ that normalizes $\check{T}$.

\section{Lemma 1.1.16.}

(1) There exists a monoidal self-equivalence $\mathrm{F}_{w}: \dot{\mathfrak{u}}_{\ell}-\bmod \rightarrow \mathfrak{\mathfrak { u }}_{\ell}$-mod that commutes with the restriction functor $\mathbf{U}_{\ell}-\bmod \rightarrow \dot{\mathfrak{u}}_{\ell}$-mod.

(2) There exists a self-equivalence $\mathrm{F}_{w}: \mathfrak{u}_{\ell}-\bmod \rightarrow \mathfrak{u}_{\ell}$-mod, compatible with the functor tensor product functor $\mathfrak{u}_{\ell}-\bmod \times \dot{\mathfrak{u}}_{\ell}-\bmod \rightarrow \mathfrak{u}_{\ell}$-mod.

We will return to the discussion of functors $\mathrm{F}_{w}$ in Sect. 1.2.5.

For an element $w \in W$ let $\dot{\mathfrak{b}}_{\ell}^{w,-}$ be the corresponding subalgebra of $\dot{\mathfrak{u}}_{\ell}$. Let us denote by ${ }^{w} \dot{M}^{\lambda}$ the $\dot{\mathfrak{u}}_{\ell}$-module induced from the $\dot{\mathfrak{b}}_{\ell}^{w,-}$-character $\mathbf{C}^{\lambda}$. For $w=1$ we recover $\dot{M}^{\lambda}$. We have:

$$
{ }^{w} \dot{M}^{w(\lambda)} \simeq \mathrm{F}_{w}\left(\dot{M}^{\lambda}\right)
$$


As in Lemma 1.1.7,

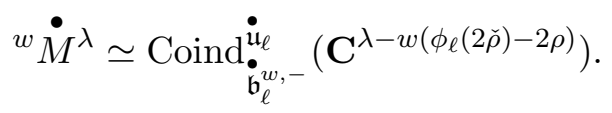

In particular, the module ${ }^{w_{0}} \dot{M}^{\lambda-\phi_{\ell}(2 \check{\rho})+2 \rho}$ is isomorphic to what is usually called the baby Verma module with highest weight $\lambda$. Since all Coind $\underset{\mathfrak{b}_{\ell}^{w,-}}{\mathfrak{u}_{\ell}}\left(\mathbf{C}^{\lambda}\right)$ have simple cosocles, we deduce that all twisted baby co-Verma modules also have simple cosocles.

\subsection{Modules over $\mathfrak{u}_{\ell}$ as Hecke-proper modules over $\mathbf{U}_{\ell}$.}

1.2.1. The Hecke categories. Following $[\mathrm{AG}]$, we introduce the category Hecke $\left(\mathbf{U}_{\ell}, \check{G}\right)$ to consist of pairs

$$
\left(M \in \mathbf{U}_{\ell}-\overline{\bmod },\left\{\alpha_{V}, \forall V \in \operatorname{Rep}(\check{G})\right\}\right),
$$

where each $\alpha_{V}$ is a map of $\mathbf{U}_{\ell \text {-modules }}$

$$
\alpha_{V}: \operatorname{Fr}(V) \otimes M \rightarrow M \otimes \underline{V}
$$

(for $V \in \operatorname{Rep}(\check{G})$, the notation $\underline{V}$ stands for the underlying vector space), such that

- For $V=\mathbb{C}, \alpha_{V}: M \rightarrow M$ is the identity map.

- For a map $V_{1} \rightarrow V_{2}$, the diagram

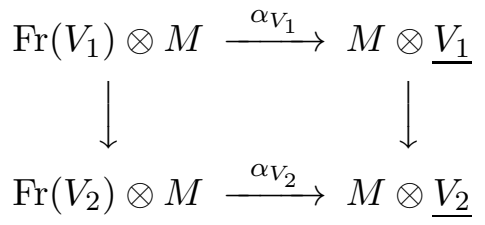

commutes.

- A compatibility with tensor products holds in the sense that the map

$$
\begin{aligned}
& \operatorname{Fr}\left(V_{1}\right) \otimes \operatorname{Fr}\left(V_{2}\right) \otimes M \rightarrow \operatorname{Fr}\left(V_{1} \otimes V_{2}\right) \otimes M \stackrel{\alpha_{V_{1} \otimes V_{2}}}{\longrightarrow} M \otimes \underline{V_{1} \otimes V_{2}} \rightarrow M \otimes \underline{V_{1}} \otimes \underline{V_{2}} \\
& \text { equals } \\
& \quad \operatorname{Fr}\left(V_{1}\right) \otimes \operatorname{Fr}\left(V_{2}\right) \otimes M \stackrel{\operatorname{id} \otimes \alpha_{V_{2}}}{\longrightarrow} \operatorname{Fr}\left(V_{1}\right) \otimes M \otimes \underline{V_{2}} \stackrel{\alpha_{V_{1}}}{\simeq} M \otimes \underline{V_{1}} \otimes \underline{V_{2}} .
\end{aligned}
$$

It was shown in $[\mathrm{AG}]$ that the maps $\alpha_{V}$ are necessarily isomorphisms.

Morphisms in this category between $\left(M, \alpha_{V}\right)$ and $\left(M^{\prime}, \alpha_{V}^{\prime}\right)$ are $\mathbf{U}_{\ell^{-} \text {-module maps }}$ $M \rightarrow M^{\prime}$ preserving the above structures. Evidently, Hecke $\left(\mathbf{U}_{\ell}, \check{G}\right)$ is an abelian category.

The main result of $[\mathrm{AG}]$ is the following theorem:

Theorem 1.2.2. The category $\operatorname{Hecke}\left(\mathbf{U}_{\ell}, \check{G}\right)$ is naturally equivalent to $\mathfrak{u}_{\ell}-\overline{\bmod }$.

We recall that the functors $\operatorname{Hecke}\left(\mathbf{U}_{\ell}, \check{G}\right) \rightleftarrows \mathfrak{u}_{\ell}-\overline{\bmod }$ are defined as follows. To $N \in \mathfrak{u}_{\ell}-\overline{\bmod }$ we attach the object in $\mathbf{U}_{\ell}-\overline{\bmod }$ by taking $\operatorname{Ind}_{\mathfrak{u}_{\ell}}^{\mathbf{U}_{\ell}}(N)$. It satisfies the Hecke condition due to Proposition 1.1.11. 
Vice versa, given an object $M$ of $\operatorname{Hecke}\left(\mathbf{U}_{\ell}, \check{G}\right)$, the restriction $\operatorname{Res}_{\mathfrak{u}_{\ell}}^{\mathbf{U}_{\ell}}(M)$ is acted on by the algebra $\mathcal{O}_{\breve{G}}$, and the corresponding object of $\mathfrak{u}_{\ell}-\overline{\bmod }$ is by definition the tensor product $\operatorname{Res}_{\mathfrak{u}_{\ell}}^{\mathbf{U}_{\ell}}(M) \underset{\mathcal{O}_{\breve{G}}}{\otimes} \mathbb{C}_{1}$, where $\mathbb{C}_{1}$ is the skyscraper at $1 \in \check{G}$.

A typical example of an object of $\operatorname{Hecke}\left(\mathbf{U}_{\ell}, \check{G}\right)$ is obtained by taking $\operatorname{Fr}\left(R_{\check{G}}\right) \otimes M$, where $R_{\breve{G}}$ is the algebra of functions on $\check{G}$, regarded as a representation of $\check{G}$, and $M \in \mathbf{U}_{\ell}-\overline{\bmod }$.

We say that an object of Hecke $\left(\mathbf{U}_{\ell}, \check{G}\right)$ is finitely generated if it admits a surjection from an object of the above form for $M \in \mathbf{U}_{\ell}$-mod. Evidently, the subcategory of finitely generated objects of Hecke $\left(\mathbf{U}_{\ell}, \check{G}\right)$, denoted Hecke $\left(\mathbf{U}_{\ell}, \check{G}\right)_{f . g}$, transforms under the equivalence of Theorem 1.2.2 to $\mathfrak{u}_{\ell}$-mod. In particular, this subcategory is Artinian, and Hecke $\left(\mathbf{U}_{\ell}, \check{G}\right)$ is the ind-completion of $\operatorname{Hecke}\left(\mathbf{U}_{\ell}, \check{G}\right)_{f . g .}$.

Consider the subcategory Hecke $\left(\mathbf{U}_{\ell}, \check{G}\right)_{0}$ of Hecke $\left(\mathbf{U}_{\ell}, \check{G}\right)$, equal to the preimage of $\mathbf{U}_{\ell}-\bmod _{0}$ under the forgetful functor. According to $[\mathrm{AG}]$, the equivalence of Theorem 1.2.2 induces an equivalence between $\operatorname{Hecke}\left(\mathbf{U}_{\ell}, \check{G}\right)_{0}$ and $\mathfrak{u}_{\ell}-\overline{\bmod }_{0}$. We will denote by Hecke $\left(\mathbf{U}_{\ell}, \breve{G}\right)_{0, f . g}$. the intersection of Hecke $\left(\mathbf{U}_{\ell}, \breve{G}\right)_{0}$ with Hecke $\left(\mathbf{U}_{\ell}, \breve{G}\right)_{f . g}$; this category is equivalent to $\mathfrak{u}_{\ell}-\bmod _{0}$.

1.2.3. Hecke categories, graded version. We define the category $\dot{H}$ ecke $\left(\mathbf{U}_{\ell}, \check{G}\right)$ as follows. Its objects are $\check{\Lambda}$-graded objects $\dot{M}=\oplus M_{\check{\nu}}$ of $\mathbf{U}_{\ell}$-mod, each endowed with a collection of grading-preserving maps $\alpha_{V}, \forall V \in \operatorname{Rep}(\check{G})$

$$
\operatorname{Fr}(V) \otimes \dot{M} \simeq \dot{M} \otimes \underline{V}
$$

(where the grading on the LHS is induced from that on $M$, and on the RHS is diagonal with respect to the action of $\check{T}$ on $\underline{V}$ ), which satisfy the same conditions as in the definition of $\operatorname{Hecke}\left(\mathbf{U}_{\ell}, \check{G}\right)$.

Maps in this category are grading preserving maps in $\mathbf{U}_{\ell}$-mod that intertwine the corresponding $\alpha_{V}$ 's. We have the following graded version of Theorem 1.2.2:

Theorem 1.2.4. The category $\dot{H}$ ecke $\left(\mathbf{U}_{\ell}, \check{G}\right)$ is equivalent to $\dot{\mathfrak{u}}_{\ell}-\overline{\bmod }$. The forgetful functor $\dot{H} \operatorname{Hecke}\left(\mathbf{U}_{\ell}, \check{G}\right) \rightarrow \operatorname{Hecke}\left(\mathbf{U}_{\ell}, \check{G}\right)$ identifies under this equivalence with $\operatorname{Res}_{\mathfrak{u}_{\ell}}^{\dot{\mathfrak{u}}_{\ell}}$.

We will denote by $\dot{M} \mapsto \dot{M}\{\check{\mu}\}$ the functor on $\dot{H}$ ecke $\left(\mathbf{U}_{\ell}, \check{G}\right)$ given by the shift of grading by $\check{\mu} \in \check{\Lambda}$. Under the equivalence of Theorem 1.2.4 this functor transforms to the functor $N \mapsto \mathbf{C}^{\check{\mu}} \otimes N$.

Let $\dot{H}$ ecke $\left(\mathbf{U}_{\ell}, \check{G}\right)_{0}$ be the preimage in $\dot{H}$ ecke $\left(\mathbf{U}_{\ell}, \check{G}\right)$ of $\mathbf{U}_{\ell}-\bmod _{0}$ under the obvious forgetful functor. This subcategory goes over under the equivalence of Theorem 1.2.4 to $\dot{\mathfrak{u}}_{\ell}-\overline{\bmod }_{0}$.

Let $\dot{R}_{\breve{G}}$ be the algebra of functions of $\check{G}$, regarded as a $\check{\Lambda}$-graded representation of $\check{G}$ (the grading comes from the action of $\check{G}$ on itself on the right). A typical example of an object of $\dot{H}$ ecke $\left(\mathbf{U}_{\ell}, \check{G}\right)$ is $\operatorname{Fr}\left(\dot{R}_{\check{G}}\right) \otimes M$ for $M$ being a $\check{\Lambda}$-graded object of $\mathbf{U}_{\ell}$-mod. 
We will denote by $\dot{H}$ ecke $\left(\mathbf{U}_{\ell}, \check{G}\right)_{f . g .}\left(\right.$ resp., $\dot{H}$ ecke $\left.\left(\mathbf{U}_{\ell}, \check{G}\right)_{0, f . g .}\right)$ the corresponding subcategories of finitely generated objects. These subcategories transform under the equivalence of Theorem 1.2.4 to $\dot{\mathfrak{u}}_{\ell}-\bmod$ and $\dot{\mathfrak{u}}_{\ell}-\bmod _{0}$, respectively.

1.2.5. Action of the dual group. Note that the equivalence of Theorem 1.2.2 makes it explicit that the category $\mathfrak{u}_{\ell}-\overline{\bmod }$ carries an action of the dual group by autoequivalences. The latter means that to every $N \in \mathfrak{u}_{\ell}-\overline{\bmod }$ we can attach a family ${ }^{\check{G}} N$ of objects of $\mathfrak{u}_{\ell}-\overline{\bmod }$, parametrized by $\check{G}$, such that the natural associativity condition holds.

The corresponding family is defined in the language of Hecke $\left(\mathbf{U}_{\ell}, \check{G}\right)$ as follows. For $\left(M,\left\{\alpha_{V}\right\}\right) \in \operatorname{Hecke}\left(\mathbf{U}_{\ell}, \check{G}\right)$ its fiber at $\mathbf{g} \in \check{G}$ is $\left(M,\left\{\mathbf{g} \cdot \alpha_{V}\right\}\right)$, where each $\mathbf{g} \cdot \alpha_{V}$ is the composition of $\alpha$ with the automorphism induced by $\mathbf{g}$ on $\underline{V}$. We will use the notation $N \mapsto \mathbf{g}_{N}$ for these functors.

Consider now the case of $\dot{\mathfrak{u}}_{\ell}-\overline{\bmod } \simeq \dot{\operatorname{Hecke}}\left(\mathbf{U}_{\ell}, \check{G}\right)$. In this case we do not have an action of the entire $\breve{G}$ on the category, but rather of the normalizer of the Cartan subgroup $\check{T}$, due to the grading condition.

Lemma 1.2.6. For a pair $\left(\mathrm{F}_{w}, w_{\ell}\right)$ as above, the functors $\mathrm{F}_{w}$

$$
\mathfrak{u}_{\ell}-\overline{\bmod } \rightarrow \mathfrak{u}_{\ell}-\overline{\bmod } \text { and } \dot{\mathfrak{u}}_{\ell}-\overline{\bmod } \rightarrow \dot{\mathfrak{u}}_{\ell}-\overline{\bmod }
$$

are naturally isomorphic to the functors $N \mapsto w_{\breve{G}} N$, where $w_{\breve{G}}$ is the corresponding element in the normalizer of $\check{T}$ in $\breve{G}$.

1.2.7. Compatibility with duality. Recall that both categories $\mathbf{U}_{\ell}$-mod and $\dot{\mathfrak{u}}_{\ell}$-mod carry a canonical self anti-equivalence (contragredient duality), $M \mapsto M^{\vee}$, compatible with the forgetful functor $\operatorname{Res}_{\mathfrak{u}_{\ell}}^{\mathbf{U}_{\ell}}$. We would like to express the duality functor on $\dot{\mathfrak{u}}_{\ell}$-mod in terms of $\dot{H}$ ecke $\left(\mathbf{U}_{\ell}, \check{G}\right)_{f . g .}$.

Thus, let $N$ be an object of $\dot{\mathfrak{u}}_{\ell}-\bmod$ and $\dot{M} \in \dot{\mathrm{H} e c k e}\left(\mathbf{U}_{\ell}, \check{G}\right)$ the object corresponding to it under Theorem 1.2.4. Since $\dot{M} \in \dot{\mathrm{H} e c k e}\left(\mathbf{U}_{\ell}, \check{G}\right)_{f . g}$, it can be represented as the cokernel of an arrow

$$
\oplus_{i} \operatorname{Fr}\left(\dot{R}_{G}\right) \otimes M_{i}^{1}\left\{\check{\mu}_{i}^{1}\right\} \rightarrow \underset{j}{\oplus} \operatorname{Fr}\left(\dot{R}_{G}\right) \otimes M_{j}^{2}\left\{\check{\mu}_{j}^{2}\right\}
$$

where the indices $i$ and $j$ run over some finite sets, and $M_{i}^{1}, M_{j}^{2}$ are objects of $\mathbf{U}_{\ell}$-mod.

An arrow as above comes from a system of maps in $\mathbf{U}_{\ell}$-mod

$$
M_{i}^{1} \rightarrow \operatorname{Fr}\left(V^{i, j}\right) \otimes M_{j}^{2} \otimes\left(\underline{V}^{i, j}\right)^{*}\left(\check{\mu}_{j}^{2}-\check{\mu}_{i}^{1}\right),
$$

where $V^{i, j}$ are some finite-dimensional representations of $\check{G}$. By adjunction, we obtain a system of maps

$$
\operatorname{Fr}\left(\left(V^{i, j}\right)^{*}\right) \otimes M_{i}^{1} \otimes \underline{V}^{i, j}\left(\check{\mu}_{j}^{1}-\check{\mu}_{i}^{2}\right) \rightarrow M_{j}^{2},
$$

and applying the duality,

$$
\left(M_{j}^{2}\right)^{\vee} \rightarrow \operatorname{Fr}\left(\left(\left(V^{i, j}\right)^{*}\right)^{\vee}\right) \otimes\left(M_{j}^{1}\right)^{\vee} \otimes\left(\underline{V}^{i, j}\right)^{*}\left(\check{\mu}_{j}^{2}-\check{\mu}_{i}^{1}\right) .
$$


Note that for a representation $V$ of $\check{G}$,

$$
\underline{V^{\vee}}(\check{\mu}) \simeq \underline{V}^{*}(-\check{\mu}) .
$$

Hence, if we set $U^{i, j}=\left(\left(V^{i, j}\right)^{*}\right)^{\vee}$, we obtain a system of maps map

$$
\left(M_{j}^{2}\right)^{\vee} \rightarrow \operatorname{Fr}\left(U^{i, j}\right) \otimes\left(M_{j}^{1}\right) \vee \otimes\left(\underline{U}^{i, j}\right)^{*}\left(\check{\mu}_{1}-\check{\mu}_{2}\right)
$$

which in turn gives rise to a map in $\dot{H}$ ecke $\left(\mathbf{U}_{\ell}, \check{G}\right)$ :

$$
\underset{j}{\oplus} \operatorname{Fr}\left(\dot{R}_{G}\right) \otimes\left(M_{j}^{2}\right) \vee\left\{\check{\mu}_{j}^{2}\right\} \rightarrow \underset{i}{\oplus} \operatorname{Fr}\left(\dot{R}_{G}\right) \otimes\left(M_{i}^{1}\right) \vee\left\{\check{\mu}_{i}^{1}\right\}
$$

Then $N^{\vee}$ corresponds to the object in $\dot{H}$ ecke $\left(\mathbf{U}_{\ell}, \check{G}\right)$ equal to the kernel of the latter map.

\subsection{Realization via the affine Grassmannian.}

1.3.1. Let $\mathrm{Gr}_{G} \simeq G((t)) / G[[t]]$ be the affine Grassmannian corresponding to $G$, and let $\operatorname{Perv}\left(\mathrm{Gr}_{G}\right)$ denote the category of perverse sheaves on it.

Let $\mathrm{Sph}_{G}$ denote the category of $G[[t]]$-equivariant perverse sheaves on $\operatorname{Gr}_{G}$. We recall that $\mathrm{Sph}_{G}$ is naturally a monoidal category that acts on $\operatorname{Perv}\left(\mathrm{Gr}_{G}\right)$ by convolution functors:

$$
\mathcal{F} \in \operatorname{Perv}\left(\mathrm{Gr}_{G}\right), \mathcal{S} \in \mathrm{Sph}_{G} \mapsto \mathcal{F} \star \mathcal{S} .
$$

Moreover, $\mathrm{Sph}_{G}$ possesses a natural commutativity constraint, and as a tensor category it is equivalent to $\operatorname{Rep}(\check{G})$. We will denote this equivalence by $V \in \operatorname{Rep}(\check{G}) \mapsto$ $\mathcal{V} \in \mathrm{Sph}_{G}$. Under this equivalence, the irreducible representation $V^{\check{\lambda}}$ goes over to $\mathcal{V}^{\check{\lambda}}=\mathrm{IC}_{\check{\lambda}, \mathrm{Gr}_{G}}$, where the latter is the IC sheaf on the closure of the orbit $\operatorname{Gr}_{G}^{\check{\lambda}}=G[[t]] \cdot \check{\lambda}$.

1.3.2. For $k \in \mathbb{N}$ we will denote by $G^{k}$ the corresponding congruence subgroup in $G[[t]]$, and by $\operatorname{Perv}\left(\mathrm{Gr}_{G}\right)^{G^{k}}$ the category of $G^{k}$-equivariant perverse sheaves on $\operatorname{Gr}_{G}$. For $k=0$ we recover $\operatorname{Sph}_{G}$; for $k>0$ this is a full subcategory of $\operatorname{Perv}\left(\operatorname{Gr}_{G}\right)$, stable under extensions, since $G^{k}$ is pro-unipotent.

Let $I$ (resp., $I^{0}$ ) be the Iwahori subgroup of $G$ (resp., its unipotent radical). We will denote by $\operatorname{Perv}\left(\mathrm{Gr}_{G}\right)^{I}$, Perv $\left(\mathrm{Gr}_{G}\right)^{I^{0}}, \mathrm{D}\left(\mathrm{Gr}_{G}\right)^{I}, \mathrm{D}\left(\mathrm{Gr}_{G}\right)^{I^{0}}$ the corresponding categories of equivariant perverse sheaves and triangulated categories.

Recall that $I$-orbits on $\mathrm{Gr}_{G}$ are parametrized by $W_{a f f} / W$, which we will identify with the set of elements in $W_{a f f}$, right-minimal with respect to $W$. Any such element $\tilde{w}$ can be uniquely written as

$$
\tilde{w}=w \cdot \check{\lambda}
$$

where $w \in W, \check{\lambda} \in \check{\Lambda}^{+}$. The condition of being right-minimal with respect to $W$ implies that whenever for some $\imath \in \mathcal{J}$, we have $\left\langle\alpha_{\imath}, \check{\lambda}\right\rangle=0$, then $w\left(\alpha_{\imath}\right) \in \Lambda^{\text {pos }}$.

For $\tilde{w}$ as above we will denote by $\mathrm{IC}_{\tilde{w}, \mathrm{Gr}_{G}}$ the IC sheaf on the closure of the corresponding $I$-orbit. By $\mathcal{W}^{*, \tilde{w}}$ (resp., $\mathcal{W}^{!}, \tilde{w}$ ) we will denote the corresponding costandard (resp., standard) objects corresponding to the extension by * (resp., !) of the constant perverse sheaf on this orbit. 
Since $I$-orbits and $I^{0}$-orbits on $\operatorname{Gr}_{G}$ coincide, the irreducibles in $\operatorname{Perv}\left(\mathrm{Gr}_{G}\right)^{I}$ are the same as in $\operatorname{Perv}\left(\mathrm{Gr}_{G}\right)^{I^{0}}$.

In the sequel we will also need some notation pertaining to the affine flag variety $\mathrm{Fl}_{G}=G((t)) / I$. We will denote by $\operatorname{Perv}\left(\mathrm{Fl}_{G}\right)$ (resp., Perv $\left.\left(\mathrm{Fl}_{G}\right)^{I}\right)$ the category of perverse (resp., I-equivariant) sheaves on $\mathrm{Fl}_{G}$, and by $\mathrm{D}\left(\mathrm{Fl}_{G}\right)$ (resp., $\left.\mathrm{D}\left(\mathrm{Fl}_{G}\right)^{I}\right)$ the corresponding triangulated category.

The category $\mathrm{D}\left(\mathrm{Fl}_{G}\right)^{I}$ has a natural monoidal structure, and it acts by convolution on $\mathrm{D}\left(\mathrm{Fl}_{G}\right)$. In addition, we have a natural convolution functor

$$
\mathrm{D}\left(\mathrm{Fl}_{G}\right) \times \mathrm{D}\left(\mathrm{Gr}_{G}\right)^{I} \rightarrow \mathrm{D}\left(\mathrm{Gr}_{G}\right) .
$$

For $\tilde{w} \in W_{\text {aff }}$ we will denote by $j_{*, \tilde{w}}$ (resp., $\left.j_{!}, \tilde{w}\right)$ the costandard (resp., standard) object in $\operatorname{Perv}\left(\mathrm{Fl}_{G}\right)^{I}$ attached to the corresponding $I$-orbit on $\mathrm{Fl}_{G}$. We have:

$$
j_{*, \tilde{w}_{1}} \star j_{*, \tilde{w}_{2}}=j_{*, \tilde{w}_{1} \cdot \tilde{w}_{2}} \text { and } j_{!}, \tilde{w}_{1} \star j_{!}, \tilde{w}_{2}=j_{!}, \tilde{w}_{1} \cdot \tilde{w}_{2},
$$

whenever $l\left(\tilde{w}_{1}\right)+l\left(\tilde{w}_{2}\right)=l\left(\tilde{w}_{1} \cdot \tilde{w}_{2}\right)$, where $l(\cdot)$ is the length function on $W_{a f f}$. Morover, if $\tilde{w}$ is right $W$-minimal,

$$
j_{*, \tilde{w}} \star \delta_{1, \mathrm{Gr}_{G}} \simeq \mathcal{W}^{*, \tilde{w}} \text { and } j_{!, \tilde{w}} \star \delta_{1, \mathrm{Gr}_{G}} \simeq \mathcal{W}^{!, \tilde{w}} .
$$

1.3.3. According to $[\mathrm{KT}]$ combined with $[\mathrm{KL}]$ (or, alternatively by $[\mathrm{ABG}]$, adapted to the even root of unity case), we have the following:

Theorem 1.3.4. There exists an equivalence of categories

$$
\text { Loc : } \mathbf{U}_{\ell}-\bmod _{0} \rightarrow \operatorname{Perv}\left(\operatorname{Gr}_{G}\right)^{I^{0}},
$$

such that the functor

$$
\mathbf{U}_{\ell}-\bmod _{0} \times \operatorname{Rep}(\check{G}) \rightarrow \mathbf{U}_{\ell}-\bmod _{0}: M, V \mapsto \operatorname{Fr}(V) \otimes M
$$

identifies with

$$
\operatorname{Perv}\left(\mathrm{Gr}_{G}\right)^{I^{0}} \times \mathrm{Sph}_{G} \rightarrow \operatorname{Perv}\left(\mathrm{Gr}_{G}\right)^{I^{0}}: \mathcal{S}, V \mapsto \mathcal{S} \star \mathcal{V}
$$

Moreover, the contragredient duality functor on $\mathbf{U}_{\ell}$-mod goes over to Verdier duality on $\operatorname{Perv}\left(\mathrm{Gr}_{G}\right)^{I^{0}}$.

Let us describe the image of irreducibles under this equivalence. If $\lambda \in \Lambda^{+}$is such that $\mathbf{L}^{\lambda} \in \mathbf{U}_{\ell}-\bmod _{0}$, we can uniquely write

$$
\lambda=\phi_{\ell}(\check{\lambda})+w^{-1}(\rho)-\rho,
$$

where $\check{\lambda} \in \check{\Lambda}$. In this case $\tilde{w}:=w \cdot \check{\lambda} \in W_{a f f}$ is right $W$-minimal. Then

$$
\operatorname{Loc}\left(\mathbf{L}^{\lambda}\right) \simeq \mathrm{IC}_{\tilde{w}, \operatorname{Gr}_{G}} \text { and } \operatorname{Loc}\left(\mathbf{W}^{\lambda}\right) \simeq \mathcal{W}^{*, \tilde{w}}
$$

Note also that a weight $\lambda$ as above is restricted if and only if the pair $(\check{\lambda}, w)$ satisfies the following:

$$
\left\{\begin{array}{l}
\left\langle\alpha_{i}, \check{\lambda}\right\rangle=0 \text { if } w\left(\alpha_{i}\right) \in \check{\Lambda}^{\text {pos }} \\
\left\langle\alpha_{i}, \check{\lambda}\right\rangle=1 \text { if }-w\left(\alpha_{i}\right) \in \check{\Lambda}^{\text {pos }} .
\end{array}\right.
$$

Hence, for each $w$, the corresponding element $\check{\lambda}$ is well-defined modulo characters of $\check{G} /[\breve{G}, \check{G}]$ (which are the same as cocharacters of $Z(G)$ ). We will make such a choice 
MODULES OVER THE SMALL QUANTUM GROUP AND SEMI-INFINITE FLAG MANIFOLD 17

and denote the corresponding irreducible in $\operatorname{Perv}\left(\mathrm{Gr}_{G}\right)^{I}$ by $\mathcal{L}^{w}$. We will assume that for $w=1, \mathcal{L}^{w}=\delta_{1, \mathrm{Gr}_{G}}$. Note that $\mathcal{L}^{w_{0}} \simeq \mathrm{IC}_{w_{0} \cdot \check{\rho}^{\prime}, \mathrm{Gr}_{G}}$, where $\check{\rho}^{\prime}$ is some element of $\check{\Lambda}$, for which $\left\langle\alpha_{\imath}, \check{\rho}^{\prime}\right\rangle=1$ for $\forall \imath \in \mathcal{J}$. Such $\check{\rho}^{\prime}$ exists due to the assumption that the center of $G$ is connected. Note that $2 \check{\rho}^{\prime}$ is not in general equal to $2 \check{\rho}$, the latter being the sum of positive coroots.

The following is a corollary of Theorem 1.1.4 combined with the equivalence of Theorem 1.3.4:

\section{Theorem 1.3.5.}

(1) For any $w$ and $\check{\mu} \in \check{\Lambda}^{+}$, the convolution $\mathcal{L}^{w} \star \mathrm{IC}_{\check{\mu}, \mathrm{Gr}_{G}}$ is irreducible and isomorphic to $\mathrm{IC}_{w \cdot(\check{\lambda}+\check{\mu}), \mathrm{Gr}_{G}}$, if $\mathcal{L}^{w}=\mathrm{IC}_{w \cdot \check{\lambda}, \mathrm{Gr}_{G}}$.

(2) Any irreducible object of $\operatorname{Perv}\left(\mathrm{Gr}_{G}\right)^{I}$ has the form $\mathcal{L}^{w} \star \mathrm{IC}_{\breve{\mu}, \mathrm{Gr}_{G}}$ for unique $w$ and $\check{\mu}$.

For completeness, in the next section we will give a purely geometric proof of this result.

1.3.6. Hecke categories. Let $\overline{\operatorname{Perv}}\left(\mathrm{Gr}_{G}\right)$ denote the ind-completion of Perv $\left(\mathrm{Gr}_{G}\right)$. Let Hecke $\left(\mathrm{Gr}_{G}, \check{G}\right)$ denote the category, whose objects are pairs

$$
\left(\mathcal{S} \in \overline{\operatorname{Perv}}\left(\operatorname{Gr}_{G}\right),\left\{\alpha_{V}, \forall V \in \operatorname{Rep}(\check{G})\right\}\right),
$$

where each $\alpha_{V}$ is a map

$$
\mathcal{S} \star V \rightarrow \underline{V} \otimes \mathcal{S}
$$

such that the collection $\left\{\alpha_{V}\right\}$ satisfies the same compatibility conditions as in the definition of Hecke $\left(\mathbf{U}_{\ell}, \check{G}\right)$. As in the case of the quantum group, one shows that the maps $\alpha_{V}$ are then automatically isomorphisms.

Morphisms between $\left(\mathcal{S}^{1},\left\{\alpha_{V}^{1}\right\}\right)$ and $\left(\mathcal{S}^{2},\left\{\alpha_{V}^{2}\right\}\right)$ are maps $\mathcal{S}^{1} \rightarrow \mathcal{S}^{2}$ that intertwine the data of $\alpha_{V}$. The category Hecke $\left(\operatorname{Gr}_{G}, \check{G}\right)$ is evidently abelian.

Let $\mathcal{R}_{\breve{G}}$ be ind-object of $\mathrm{Sph}_{G}$, corresponding under the equivalence $\operatorname{Rep}(\check{G}) \simeq \operatorname{Sph}_{G}$ to $R_{\breve{G}}$. A typical example of an object of Hecke $\left(\mathrm{Gr}_{G}, \check{G}\right)$ is obtained by setting

$$
\mathcal{S}:=\mathcal{S}^{1} \star \mathcal{R}_{\breve{G}}
$$

for $\mathcal{S}^{1} \in \overline{\operatorname{Perv}}\left(\operatorname{Gr}_{G}\right)$, where the Hecke isomorphisms come from the canonical isomorphisms

$$
\mathcal{R}_{\breve{G}} \star V \simeq \underline{V} \otimes \mathcal{R}_{\breve{G}} .
$$

As in the case of Hecke $\left(\mathbf{U}_{\ell}, \check{G}\right)$, the category Hecke $\left(\mathrm{Gr}_{G}, \check{G}\right)$ is naturally acted on by the group $\check{G}$.

We say that an objet of Hecke $\left(\mathrm{Gr}_{G}, \check{G}\right)$ is finitely generated if it admits a surjection from an object of the form $\mathcal{S}^{1} \star \mathcal{R}_{\breve{G}}$ with $\mathcal{S}^{1} \in \operatorname{Perv}\left(\mathrm{Gr}_{G}\right)$. This condition is equivalent to the fact that the functor of Hom from this object commutes with direct sums.

Conjecture 1.3.7. A sub-object of a finitely generated object of Hecke $\left(\mathrm{Gr}_{G}, \breve{G}\right)$ is finitely generated. 
We will denote by Hecke $\left(\operatorname{Gr}_{G}, \check{G}\right)^{G^{k}}$ (resp., Hecke $\left(\mathrm{Gr}_{G}, \check{G}\right)^{I^{0}}$, Hecke $\left.\left(\operatorname{Gr}_{G}, \check{G}\right)^{I}\right)$ a version of the above category, where $\mathcal{S}$ is assumed to be an object of the ind-completion of the corresponding category $\operatorname{Perv}\left(\mathrm{Gr}_{G}\right)^{G^{k}}$ (resp., Perv $\left(\mathrm{Gr}_{G}\right)^{I^{0}}, \operatorname{Perv}\left(\mathrm{Gr}_{G}\right)^{I}$ ).

As we shall see shortly, a particular case of Conjecture 1.3.7, concerning $\operatorname{Perv}\left(\operatorname{Gr}_{G}\right)^{I^{0}}$, follows easily from Theorem 1.3.5.

We introduce a graded version $\dot{H}$ ecke $\left(\mathrm{Gr}_{G}, \check{G}\right)$ of Hecke $\left(\mathrm{Gr}_{G}, \check{G}\right)$ analogously to the definition of $\dot{H}$ ecke $\left(\mathbf{U}_{\ell}, \check{G}\right)$ : its objects are pairs $\left(\dot{\mathcal{S}},\left\{\alpha_{V}\right\}\right)$, where $\dot{\mathcal{S}}$ is a $\check{\Lambda}$-graded object of $\overline{\operatorname{Perv}}\left(\operatorname{Gr}_{G}\right)$, and the maps $\alpha_{V}$ preserve the gradings on both sides. Similarly, we introduce the categories $\dot{H e c k e}\left(\mathrm{Gr}_{G}, \check{G}\right)^{G^{k}}$, $\dot{\text { Hecke }}\left(\mathrm{Gr}_{G}, \check{G}\right)^{I^{0}}, \dot{\operatorname{Hecke}}\left(\mathrm{Gr}_{G}, \check{G}\right)^{I}$.

All of these categories are acted on naturally by the normalizer of $\breve{T}$ in $\check{G}$.

Let $\dot{\mathcal{R}}_{\check{G}}$ denote the same thing as $\mathcal{R}_{\check{G}}$, where we regard it as graded via the rightaction of $\check{G}$ on $R_{\check{G}}$. A typical example of an object of $\dot{H}$ ecke $\left(\mathrm{Gr}_{G}, \check{G}\right)$ is obtained by taking $\mathcal{S}^{1} \star \dot{\mathcal{R}}_{\breve{G}}\{\check{\mu}\}$ for $\mathcal{S}^{1} \in \overline{\operatorname{Perv}}\left(\mathrm{Gr}_{G}\right)$, where $\{\check{\mu}\}$ denotes the shift of the grading functor.

In what follows we will state the results explicitly for $\dot{\operatorname{Hecke}}\left(\mathrm{Gr}_{G}, \check{G}\right)$ and its versions; the transcription to the case of $\operatorname{Hecke}\left(\mathrm{Gr}_{G}, \check{G}\right)$ is straightforward.

1.3.8. Consider now the category $\operatorname{Hecke}\left(\mathrm{Gr}_{G}, \check{G}\right)^{I^{0}}$. Combining Theorem 1.3.4 with Theorem 1.2.4 we obtain:

Theorem 1.3.9. The category $\dot{\mathrm{H}}_{\mathrm{ecke}}\left(\mathrm{Gr}_{G}, \check{G}\right)^{I^{0}}$ is equivalent to $\dot{\mathfrak{u}}_{\ell}-\overline{\bmod }_{0}$.

In particular, we obtain:

\section{Corollary 1.3.10.}

(1) The irreducibles in $\dot{\text { Hecke }}\left(\mathrm{Gr}_{G}, \check{G}\right)^{I^{0}}$ are of the form $\mathcal{L}^{w} \star \dot{\mathcal{R}}_{\breve{G}}\{\check{\mu}\}$ for some $w \in W$ and $\check{\mu} \in \check{\Lambda}$.

(2) Every finitely generated object in $\dot{\mathrm{Hecke}}\left(\mathrm{Gr}_{G}, \check{G}\right)^{I^{0}}$ is Artinian.

We will now give a geometric proof of this fact, using Theorem 1.3.5.

Proof. Let us first see that any map $\dot{\mathcal{S}} \rightarrow \mathcal{L}^{w} \star \dot{\mathcal{R}}_{\breve{G}}\{\check{\mu}\}$ is necessarily a surjection. (This would imply that $\mathcal{L}^{w} \star \dot{\mathcal{R}}_{\breve{G}}\{\check{\mu}\}$ is irreducible.)

With no restriction of generality, we can assume that $\dot{\mathcal{S}}$ has the form $\mathcal{S}^{\prime} \star \dot{\mathcal{R}}_{\check{G}}\left\{\check{\mu}^{\prime}\right\}$ for some $\mathcal{S}^{\prime} \in \operatorname{Perv}\left(\operatorname{Gr}_{G}\right)^{I^{0}}, \check{\mu}^{\prime} \in \check{\Lambda}$. Moreover, we can assume that $\mathcal{S}^{\prime}$ is itself irreducible. Then, by Theorem 1.3.5, $\mathcal{S}^{\prime} \simeq \mathcal{L}^{w^{\prime}} \star \mathrm{IC}_{\check{\lambda}, \mathrm{Gr}_{G}}$ for some $w^{\prime} \in W, \check{\lambda} \in \check{\Lambda}^{\text {pos }}$. Hence,

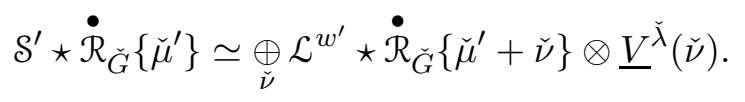

Again, by Theorem 1.3.5, the existence of a map

$$
\mathcal{L}^{w^{\prime}} \star \dot{\mathcal{R}}_{\breve{G}}\left\{\check{\mu}^{\prime}\right\} \otimes \underline{V}^{\check{\lambda}} \rightarrow \mathcal{L}^{w} \star \dot{\mathcal{R}}_{\breve{G}}\{\check{\mu}\}
$$


forces that $w^{\prime}=w$ and the map factors through the direct summand corresponding to $\check{\mu}^{\prime}+\check{\nu}=\check{\mu}$ and a linear functional $\underline{V}^{\check{\lambda}}(\check{\nu}) \rightarrow \mathbb{C}$. Such a map is manifestly surjective.

The same argument shows that any irreducible object of $\dot{H}$ ecke $\left(\operatorname{Gr}_{G}, \check{G}\right)^{I^{0}}$ admits a map from some $\mathcal{L}^{w} \star \dot{\mathcal{R}}_{\breve{G}}\{\check{\mu}\}$. This establishes the first point of the corollary.

To prove the second point, it suffices to show that the objects of the form $\mathcal{S}^{\prime} \star \dot{\mathcal{R}}_{\breve{G}}\{\check{\mu}\}$, $\mathcal{S}^{\prime} \in \operatorname{Perv}\left(\operatorname{Gr}_{G}\right)^{I^{0}}$ have finite lengths. For that we can assume that $\mathcal{S}^{\prime}$ is irreducible, and our assertion follows from (1).

Let $\dot{H}$ ecke $\left(\mathrm{Gr}_{G}, \check{G}\right)_{\text {Art }}^{I^{0}}$ denote the subcategory of Artinian (or, equivalently, finitely generated) objects of $\dot{H}$ ecke $\left(\operatorname{Gr}_{G}, \check{G}\right)^{I^{0}}$. By Theorem 1.3.9, it is equivalent to the category $\dot{\mathfrak{u}}_{\ell}-\bmod _{0}$. Hence, it also carries a duality functor, denoted $\mathbb{D}$.

Explicitly, this functor is determined by the fact that it is exact;

$$
\mathbb{D}\left(\mathcal{S}^{\prime} \star \dot{\mathcal{R}}_{\check{G}}\{\check{\mu}\}\right) \simeq \mathbb{D}\left(\mathcal{S}^{\prime}\right) \star \dot{\mathcal{R}}_{\check{G}}\{\check{\mu}\}
$$

it is extended to the entire $\dot{H}$ ecke $\left(\mathrm{Gr}_{G}, \check{G}\right)_{\text {Art }}^{I^{0}}$ by the procedure described in Sect. 1.2.7.

This functor goes over to the functor $N \mapsto N^{\vee}$ on $\mathfrak{\mathfrak { u }}_{\ell}-\bmod _{0}$, since the equivalence of Theorem 1.3.4 transforms contragredient duality to Verdier duality.

\section{Some Results on Perv $\left(\mathrm{Gr}_{G}\right)$}

\subsection{Proof of Theorem 1.3.5.}

2.1.1. It clear that point (1) of the theorem implies point (2). Indeed, for any $\mathrm{IC}_{w \cdot \check{\nu}, \operatorname{Gr}_{G}} \in \operatorname{Perv}\left(\mathrm{Gr}_{G}\right)^{I}$ define $\mathcal{J} \subset \mathcal{J}$ to be the subset of simple roots, for which $w\left(\alpha_{\imath}\right) \in \check{\Lambda}^{\text {pos }}$. Define

$$
\check{\lambda}^{\prime}:=\check{\nu}-\sum_{\jmath \in \mathcal{J}}\left\langle\alpha_{\jmath}, \check{\nu}\right\rangle \cdot \check{\omega}_{\jmath}-\sum_{\imath \in \mathcal{J}-\mathcal{J}}\left(\left\langle\alpha_{\imath}, \check{\nu}\right\rangle-1\right) \cdot \check{\omega}_{\imath},
$$

where $\check{\omega}_{\imath}$ are (some choice of) fundamental coweights.

Then $w \cdot \check{\lambda}^{\prime}$ is left-minimal with respect to $W$, and

$$
\mathrm{IC}_{w \cdot \check{\lambda}^{\prime}, \mathrm{Gr}_{G}} \simeq \mathcal{L}^{w} \star \delta_{\check{\eta}, \mathrm{Gr}_{G}}
$$

where $\check{\eta}$ is a co-character of $Z(G)$ and $\delta_{\check{\eta}, \mathrm{Gr}_{G}}$ is the $\delta$-function at the corresponding point of $\mathrm{Gr}_{G}$.

By point (1),

$$
\mathcal{L}^{w} \star\left(\mathrm{IC}_{\check{\nu}-\check{\lambda}^{\prime}, \mathrm{Gr}_{G}} \star \delta_{-\check{\eta}, \mathrm{Gr}_{G}}\right) \simeq \mathrm{IC}_{w \cdot \check{\nu}, \mathrm{Gr}_{G}} \cdot
$$


2.1.2. The assertion of point (1) is equivalent to the fact that $\operatorname{End}\left(\mathcal{L}^{w} \star \mathrm{IC}_{\breve{\mu}, \mathrm{Gr}_{G}}\right) \simeq \mathbb{C}$. By adjunction, this is equivalent to the fact that if $\mathcal{L}^{w}=\mathrm{IC}_{w \cdot \check{\lambda}, \operatorname{Gr}_{G}}$, then

$$
\operatorname{Hom}\left(\mathrm{IC}_{w \cdot \check{\lambda}, \mathrm{Gr}_{G}}, \mathrm{IC}_{w \cdot \check{\lambda}, \mathrm{Gr}_{G}} \star \mathrm{IC}_{\breve{\mu}, \mathrm{Gr}_{G}} \star \mathrm{IC}_{-w_{0}(\check{\mu}), \mathrm{Gr}_{G}}\right) \simeq \mathbb{C}
$$

By decomposing $\mathrm{IC}_{\breve{\mu}, \mathrm{Gr}_{G}} \star \mathrm{IC}_{-w_{0}(\check{\mu}), \mathrm{Gr}_{G}}$ as a sum of irreducibles, we arrive to the conclusion that it is enough to show that

$$
\operatorname{Hom}\left(\mathrm{IC}_{w \cdot \check{\nu}, \mathrm{Gr}_{G}}, \mathrm{IC}_{w \cdot \check{\lambda}, \mathrm{Gr}_{G}} \star \mathrm{IC}_{\check{\mu}, \mathrm{Gr}_{G}}\right) \neq 0 \Rightarrow \check{\nu}=\check{\lambda}+\check{\mu} .
$$

Note that this would automatically imply that

$$
\operatorname{Hom}\left(\mathrm{IC}_{w^{\prime} \cdot \check{\nu}, \mathrm{Gr}_{G}}, \mathrm{IC}_{w \cdot \check{\lambda}, \mathrm{Gr}_{G}} \star \mathrm{IC}_{\check{\mu}, \mathrm{Gr}_{G}}\right)=0 \text { for } w^{\prime} \neq w
$$

a fact that will be used later on.

2.1.3. We will establish (2) by analyzing the convolution diagram. First, we need to recall why the convolution functor $\operatorname{Perv}\left(\mathrm{Gr}_{G}\right) \times \operatorname{Sph}_{G} \rightarrow \operatorname{Perv}\left(\mathrm{Gr}_{G}\right)$ is exact.

Let

$$
\mathrm{Gr}_{G} \star \mathrm{Gr}_{G} \simeq G((t)) \underset{G[[t]]}{\times} \mathrm{Gr}_{G}
$$

be the convolution diagram, which we think of as fibered over $\mathrm{Gr}_{G}$ by means of projection to the first factor, with typical fiber $\mathrm{Gr}_{G}$, which we think of as the second factor. We will denote by $\pi$ the map $\mathrm{Gr}_{G} \star \mathrm{Gr}_{G} \rightarrow \mathrm{Gr}_{G}$ given by multiplication. This ind-scheme is acted on by $G((t))$, and the map $\pi$ is evidently $G((t))$-equivariant.

For $G[[t]]$-orbits $\operatorname{Gr}_{G}^{\check{\mu}_{1}}, \operatorname{Gr}_{G}^{\check{\mu}_{2}} \subset \operatorname{Gr}_{G}$ we will denote by $\operatorname{Gr}_{G}^{\check{\mu}_{1}} \star \operatorname{Gr}_{G}^{\check{\mu}_{2}}$ the corresponding locally closed subset in $\mathrm{Gr}_{G} \star \mathrm{Gr}_{G}$, which is fibered over $\operatorname{Gr}_{G}^{\check{\mu}_{1}}$ with typical fiber $\operatorname{Gr}_{G}^{\check{\mu}_{2}}$. We will denote by $\left(\operatorname{Gr}_{G} \star \mathrm{Gr}_{G}\right)^{\check{\mu}}$ (resp., $\left.\left(\mathrm{Gr}_{G}^{\check{\mu}_{1}} \star \mathrm{Gr}_{G}^{\check{\mu}_{2}}\right)^{\check{\mu}}\right)$ the preimage of $\operatorname{Gr}_{G}^{\check{\mu}}$ in $\mathrm{Gr}_{G} \star \mathrm{Gr}_{G}$ (resp., $\mathrm{Gr}_{G}^{\check{\mu}_{1}} \star \mathrm{Gr}_{G}^{\check{\mu}_{2}}$ ) under the map $\pi$.

We recall the following dimension estimates:

$$
\operatorname{dim}\left(\operatorname{Gr}_{G}^{\check{\mu}}\right)=\langle 2 \rho, \check{\mu}\rangle, \operatorname{dim}\left(\left(\operatorname{Gr}_{G}^{\check{\mu}_{1}} \star \operatorname{Gr}_{G}^{\check{\mu}_{2}}\right)^{\check{\mu}}\right)=\left\langle\rho, \check{\mu}_{1}+\check{\mu}_{2}+\check{\mu}\right\rangle
$$

Hence, the dimsnion of the fibers of the map

$$
\pi_{\check{\mu}_{1}, \check{\mu}_{2}}^{\check{\mu}}:\left(\operatorname{Gr}_{G}^{\check{\mu}_{1}} \star \operatorname{Gr}_{G}^{\check{\mu}_{2}}\right)^{\check{\mu}} \rightarrow \operatorname{Gr}_{G}^{\check{\mu}}
$$

is $\leq\left\langle\rho, \check{\mu}_{1}+\check{\mu}_{2}-\check{\mu}\right\rangle$.

For $\mathcal{S} \in \operatorname{Perv}\left(\mathrm{Gr}_{G}\right), \mathcal{F} \in \mathrm{Sph}_{G}$, we will denote by $\mathcal{S} \widetilde{\otimes} \mathcal{F}$ the corresponding perverse sheaf on $\mathrm{Gr}_{G} \star \mathrm{Gr}_{G}$, and by definition,

$$
\mathcal{S} \star \mathcal{F}=\pi_{!}(\mathcal{S} \widetilde{\otimes} \mathcal{F}) .
$$

To prove the exactness of convolution, by Verdier duality, it suffices to show that the *-restriction of $\mathcal{S} \widetilde{\nabla} \mathcal{F}$ to every $\left(\mathrm{Gr}_{G}^{\check{\mu}_{1}} \star \mathrm{Gr}_{G}^{\breve{\mu}_{2}}\right)^{\check{\mu}}$ lives in the cohomological degrees $-\leq\left\langle\rho, \check{\mu}_{1}+\check{\mu}_{2}-\check{\mu}\right\rangle$. 
It is evident that the *-restriction of $\mathcal{S} \widetilde{\nabla} \mathcal{F}$ to $\mathrm{Gr}_{G}^{\check{\mu}_{1}} \star \mathrm{Gr}_{G}^{\check{\mu}_{2}}$ lives in the cohomological degrees $\leq 0$. Moreover, $\left.\mathcal{S} \widetilde{\otimes} \mathcal{F}\right|_{\mathrm{Gr}_{G}^{\mu_{1}} \star \mathrm{Gr}_{G}^{\tilde{\mu}_{2}}}$ is a pull-back of a complex on the base $\mathrm{Gr}_{G}^{\check{\mu}_{1}}$.

Observe now that the constant sheaf $\underline{\mathbb{C}}\left(\operatorname{Gr}_{G}^{\check{\mu}_{1}} \star \operatorname{Gr}_{G}^{\check{\mu}_{2}}\right)$, thought of as a complex on $\operatorname{Gr}_{G}^{\check{\mu}_{1}} \star \operatorname{Gr}_{G}^{\check{\mu}_{2}}$, is universally locally acyclic (ULA) with respect to $\pi_{\check{\mu}_{1}, \check{\mu}_{2}}^{\check{\check{L}}}$. Indeed, it is $G[[t]]$-equivariant, and this group acts transitively on the base. Hence,

$$
\mathcal{S} \widetilde{\nabla} \mathcal{F}\left|\left(\operatorname{Gr}_{G}^{\check{\mu}_{1}} \star \operatorname{Gr}_{G}^{\check{\mu}_{2}}\right)^{\check{\mu}} \simeq \mathcal{S} \widetilde{\nabla} \mathcal{F}\right|_{\operatorname{Gr}_{G}^{\check{\mu}_{1}} \star \operatorname{Gr}_{G}^{\check{\mu}_{2}}} \otimes \underline{\mathbb{C}}\left(\operatorname{Gr}_{G}^{\check{\mu}_{1}} \star \operatorname{Gr}_{G}^{\check{\mu}_{2}}\right)^{\check{\mu}}
$$

lives in the cohomological degrees

$$
\leq-\operatorname{codim}\left(\left(\operatorname{Gr}_{G}^{\check{\mu}_{1}} \star \operatorname{Gr}_{G}^{\check{\mu}_{2}}\right)^{\check{\mu}}, \operatorname{Gr}_{G}^{\check{\mu}_{1}} \star \operatorname{Gr}_{G}^{\check{\mu}_{2}}\right) \leq-\left\langle\rho, \check{\mu}_{1}+\check{\mu}_{2}-\check{\mu}\right\rangle,
$$

which is what we needed.

The same argument proves also the following. Let $y \subset \operatorname{Gr}_{G}^{\check{\mu}}$ be a locally closed subscheme. In order for $\mathcal{S} \star \mathcal{F} \mid y$ to have a non-zero 0 -th perverse cohomology, it is necessary that there exist $\check{\mu}^{1}$ and $\check{\mu}^{2}$, such that the fibers of the map

$$
\operatorname{supp}\left(h^{0}\left(\left.\mathcal{S}\right|_{\left.\operatorname{Gr}_{G}^{\check{\mu}_{1}}\right)} \widetilde{\otimes} h^{0}\left(\left.\mathcal{F}\right|_{\operatorname{Gr}_{G}^{\check{\mu}_{2}}}\right)\right) \cap\left(\pi_{\check{\mu}_{1}, \check{\mu}_{2}}^{\check{\check{L}}}\right)^{-1}(y) \rightarrow y\right.
$$

are of dimension equal to $\left\langle\rho, \check{\mu}_{1}+\check{\mu}_{2}-\check{\mu}\right\rangle$, i.e., saturating the upper bound given above.

2.1.4. Thus, to prove (2), we must show that the fibers of the map

$$
\pi^{-1}((I \cdot(w \cdot \check{\nu}))) \cap\left((I \cdot(w \cdot \check{\lambda})) \star \operatorname{Gr}_{G}^{\check{\mu}}\right) \rightarrow(I \cdot(w \cdot \check{\nu})) .
$$

have dimensions $\langle\langle\rho, \check{\lambda}+\check{\mu}-\check{\nu}\rangle$ unless $\check{\nu}=\check{\lambda}+\check{\mu}$. (In the latter case the map in question is clearly one-to-one.)

Consider the orbit of the group $\operatorname{Ad}_{w \cdot w_{0}}(N((t)))$ in $\operatorname{Gr}_{G}$ passing through the point $w \cdot \check{\nu}$. Its preimage in

$$
(I \cdot(w \cdot \check{\lambda})) \star \operatorname{Gr}_{G}^{\check{\mu}} \subset \operatorname{Gr}_{G} \star \operatorname{Gr}_{G}
$$

is the union over the parameters $\check{\nu}^{\prime}$ of schemes

$$
\left(\left(\operatorname{Ad}_{w \cdot w_{0}} N((t)) \cdot w\left(\check{\nu}^{\prime}\right)\right) \cap(I \cdot(w \cdot \check{\lambda}))\right) \star\left(\left(\operatorname{Ad}_{w \cdot w_{0}} N((t)) \cdot\left(w \cdot\left(\check{\nu}-\check{\nu}^{\prime}\right)\right)\right) \cap \operatorname{Gr}_{G}^{\check{\mu}}\right),
$$

each of which is fibered over

$$
\left(N((t)) \cdot w_{0}\left(\check{\nu}^{\prime}\right)\right) \cap\left(\operatorname{Ad}_{\left(w \cdot w_{0}\right)^{-1}}(I) \cdot w_{0}(\check{\lambda})\right) \subset \operatorname{Gr}_{G}^{\check{\lambda}},
$$

with the typical fiber $\operatorname{Ad}_{w \cdot w_{0}} N((t)) \cdot\left(w \cdot\left(\check{\nu}-\check{\nu}^{\prime}\right)\right) \cap \operatorname{Gr}_{G}^{\check{\mu}}$.

Since the intersection $\operatorname{Ad}_{w \cdot w_{0}} N((t)) \cdot(w \cdot \check{\nu}) \cap I \cdot(w \cdot \check{\nu})$ consists of a single point, namely, $w \cdot \check{\lambda}$, the preimage of this point in $(I \cdot(w \cdot \check{\lambda})) \star \operatorname{Gr}_{G}^{\check{\mu}}$ injects into the variety (5). The dimension of this variety is a priori $\leq$ than

$$
\left\langle\rho,-\check{\nu}^{\prime}+\check{\lambda}\right\rangle=\langle\rho, \check{\lambda}+\check{\mu}-\check{\nu}\rangle-\left\langle\rho, \check{\mu}-\check{\nu}+\check{\nu}^{\prime}\right\rangle .
$$


The non-emptiness condition on $\left(\operatorname{Ad}_{w \cdot w_{0}} N((t)) \cdot\left(w \cdot\left(\check{\nu}-\check{\nu}^{\prime}\right)\right)\right) \cap \operatorname{Gr}_{G}^{\check{\mu}}$ implies that $\left\langle\rho, \breve{\mu}-\check{\nu}+\check{\nu}^{\prime}\right\rangle \geq 0$, and the equality is achieved only for $\check{\nu}-\check{\nu}^{\prime}=\check{\mu}$. Hence, it is sufficient to prove that the variety in (5) has dimension equal to $\left\langle\rho,-\check{\nu}^{\prime}+\check{\lambda}\right\rangle$ only for $\check{\nu}^{\prime}=\check{\lambda}$.

Note that the condition on $\check{\lambda}$ implies that

$$
\operatorname{Ad}_{\left(w \cdot w_{0}\right)^{-1}}(I) \cdot w_{0}(\check{\lambda}) \subset N^{-}[[t]]^{\prime} \cdot w_{0}(\check{\lambda}),
$$

where $N^{-}[[t]]^{\prime}$ is the preimage under $N^{-}[[t]] \rightarrow N^{-}$of $\left[N^{-}, N^{-}\right] \subset N^{-}$. Let $\Psi_{0}$ be a non-degenerate character on $N^{-}((t))$ with conductor 0 . Again, by the condition on $\check{\lambda}$,

$$
\operatorname{Ad}_{-w_{0}(\check{\lambda})}\left(N^{-}[[t]]^{\prime}\right) \subset \operatorname{ker}\left(\Psi_{0}\right) .
$$

Hence, the required assertion follows from the next result:

Proposition 2.1.5. The intersection

$$
(N((t)) \cdot \check{\mu}) \cap\left(\operatorname{ker}\left(\Psi_{0}\right) \cdot 1_{\mathrm{Gr}_{G}}\right) \subset(N((t)) \cdot \check{\mu}) \cap\left(N^{-}((t)) \cdot 1_{\mathrm{Gr}_{G}}\right) \subset \operatorname{Gr}_{G}
$$

has dimension $<\langle\rho, \check{\mu}\rangle$ unless $\check{\mu}=0$.

2.1.6. Proof of Proposition 2.1.5. The assertion of the proposition is equivalent to the fact that the character $\Psi_{0}$ is non-trivial on every connected component of the intersection

$$
(N((t)) \cdot \check{\mu}) \cap\left(N^{-}((t)) \cdot 1_{\mathrm{Gr}_{G}}\right) .
$$

Let $\check{\lambda} \in \check{\Lambda}^{+}$be a large. Then, then it is well-known that

$$
(N((t)) \cdot(\check{\lambda}+\check{\mu})) \cap\left(N^{-}((t)) \cdot \check{\lambda}\right)=\operatorname{Gr}_{G}^{\check{\lambda}+\check{\mu}} \cap\left(N^{-}((t)) \cdot \check{\lambda}\right) .
$$

Hence, it is sufficient to show that a character $\Psi_{\check{\lambda}}$ on $N^{-}((t))$ with conductor $\check{\lambda}$ is non-constant on every connected component of the intersection $\operatorname{Gr}_{G}^{\check{\lambda}+\check{\mu}} \cap\left(N^{-}((t)) \cdot \check{\lambda}\right)$. But the latter readily follows from the (top cohomology part) of the Casselman-Shalika formula, [FGV], Sect. 7.1.7.

\subsection{The baby Whittaker category.}

2.2.1. Let us denote by $I^{-}$the group $\operatorname{Ad}_{w_{0}}\left(I^{0}\right) \subset G[[t]]$, and let $\psi: I^{-} \rightarrow \mathbb{G}_{a}$ a nondegenerate character. We introduce the (baby Whittaker) category $\operatorname{Perv}\left(\operatorname{Gr}_{G}\right)^{I^{-}, \psi}$ as the that of $\left(I^{-}, \psi\right)$-equivariant perverse sheaves on $\mathrm{Gr}_{G} \cdot{ }^{4}$

If $\mathcal{S} \in \operatorname{Perv}\left(\mathrm{Gr}_{G}\right)^{I^{-}, \psi}$ and $\check{\lambda} \in \check{\Lambda}^{+}$, both *- and !- restrictions of $\left.\mathcal{S}\right|_{\operatorname{Gr}_{G}^{\check{\lambda}}}$ can be nonzero only if $\check{\lambda}$ is regular. Moreover, in this case, these restrictions are supported on the $I^{-}$-orbit of the point $w_{0}(\check{\lambda}) \in \mathrm{Gr}_{G}$.

For $\check{\lambda} \in \check{\Lambda}^{+}$we will denote by $\mathrm{IC}_{\check{\lambda}, \mathrm{Gr}_{G}}^{\psi}$ the Goresky-MacPherson extention of the $\left(I^{-}, \psi\right)$-character sheaf on the $I^{-}$-orbit of $w_{0}\left(\check{\lambda}+\check{\rho}^{\prime}\right) \in \mathrm{Gr}_{G}$. It is easy to see that the $\mathrm{IC}_{\tilde{\lambda}, \mathrm{Gr}_{G}}^{\psi}$ 's are the irreducibles of $\operatorname{Perv}\left(\mathrm{Gr}_{G}\right)^{I^{-}, \psi}$.

\footnotetext{
${ }^{4}$ The term "baby Whittaker" refers to the fact that we are imposing equivariance with respect to $I^{-}$, rather than with respect to the group ind-scheme $N^{-}((t))$.
} 
We will denote $\mathrm{IC}_{0, \mathrm{Gr}_{G}}^{\psi}$ simply by $\mathrm{IC}_{\mathrm{Gr}_{G}}^{\psi}$. It is easy to see that $\mathrm{IC}_{0, \mathrm{Gr}_{G}}^{\psi}$ is in fact a clean extension of the corresponding character sheaf on $I^{-} \cdot w_{0}\left(\check{\rho}^{\prime}\right)$. Indeed, all $G[[t]]$-orbits in the closure of $\mathrm{Gr}_{G}^{\breve{\rho}^{\prime}}$ correspond to non-regular coweights.

Using the same argument as in the proof of Theorem 1.3.5, one shows:

Theorem 2.2.2. $\mathrm{IC}_{\mathrm{Gr}_{G}}^{\psi} \star \mathrm{IC}_{\check{\lambda}, \mathrm{Gr}_{G}} \simeq \mathrm{IC}_{\check{\lambda}, \mathrm{Gr}_{G}}^{\psi}$.

The same argument as in [FGV], Sect. 6, implies then the following:

\section{Corollary 2.2.3.}

(1) The category $\operatorname{Perv}\left(\mathrm{Gr}_{G}\right)^{I^{-}, \psi}$ is semi-simple and equivalent to $\mathrm{Sph}_{G}$ by means of $\mathcal{F} \mapsto \mathrm{IC}_{\mathrm{Gr}_{G}}^{\psi} \star \mathcal{F}$.

(2) $\mathrm{IC}_{\tilde{\lambda}, \mathrm{Gr}_{G}}^{\psi}$ equals both the !- and *-extension of the corresponding character sheaf on $I^{-} \cdot w_{0}\left(\check{\lambda}+\check{\rho}^{\prime}\right)$.

2.2.4. Let $\mathrm{D}\left(\mathrm{Gr}_{G}\right)^{I^{-}, \psi}$ denote the $\left(I^{-}, \psi\right)$-equivariant derived category on $\mathrm{Gr}_{G}$. The forgetful functor $\operatorname{Perv}\left(\mathrm{Gr}_{G}\right)^{I^{-}, \psi} \rightarrow \operatorname{Perv}\left(\mathrm{Gr}_{G}\right)$ admits natural left and right adjoints, denoted $\mathrm{Av}_{!, I^{-}, \psi}$ and $\mathrm{Av}_{*, I^{-}, \psi}$, respectively.

Proposition 2.2.5. The functors $\mathrm{Av}_{!, I^{-}, \psi}[-\operatorname{dim}(\mathfrak{n})]$ and $\mathrm{Av}_{*, I^{-}, \psi}[\operatorname{dim}(\mathfrak{n})]$, when restricted to $\mathrm{D}\left(\mathrm{Gr}_{G}\right)^{I^{0}}$, are isomorphic. Both these functors are exact.

Proof. Note that the character $\psi$ factors through the map $\mathrm{I}^{-} \rightarrow \mathrm{N}^{-}$; we will denote by the same symbol $\psi$ the resulting character of $N^{-}$. Let $\psi_{N^{-}}$denote the corresponding character sheaf on $N^{-}$.

It is clear that the restrictions of $\mathrm{Av}_{!, I^{-}, \psi}$ and $\mathrm{Av}_{*, I^{-}, \psi}$ to $\mathrm{D}\left(\mathrm{Gr}_{G}\right)^{G^{1}}$ are the functors

$$
\mathcal{S} \mapsto \psi_{N^{-}} \stackrel{!}{\star} \mathcal{S}[\operatorname{dim}(\mathfrak{n})] \text { and } \mathcal{S} \mapsto \psi_{N^{-}} * \mathcal{*} \mathcal{S}[-\operatorname{dim}(\mathfrak{n})] \text {, }
$$

respectively, where $\stackrel{!}{\star}$ and $\stackrel{*}{\star}$ are the two convolution functors

$$
\mathrm{D}(G) \times \mathrm{D}\left(\mathrm{Gr}_{G}\right)^{G^{1}} \rightarrow \mathrm{D}\left(\mathrm{Gr}_{G}\right)^{G^{1}} .
$$

In particular, we have a map of functors

$$
\left.\left.\operatorname{Av}_{!, I^{-}, \psi}[-\operatorname{dim}(\mathfrak{n})]\right|_{\mathrm{D}\left(\mathrm{Gr}_{G}\right)^{G^{1}}} \rightarrow \operatorname{Av}_{*, I^{-}, \psi}[\operatorname{dim}(\mathfrak{n})]\right|_{\mathrm{D}\left(\mathrm{Gr}_{G}\right)^{G^{1}}} .
$$

To show that the above map of functors is an isomorphism, when restricted further to $\mathrm{D}\left(\mathrm{Gr}_{G}\right)^{I^{0}}$, it is sufficient to prove the corresponding fact for $\mathrm{D}\left(\mathrm{Gr}_{G}\right)^{I}{ }^{5}$

Let $\operatorname{Perv}(G / B)^{N^{-}, \psi}$ (resp., $\mathrm{D}(G / B)^{N^{-}, \psi}$ ) be the corresponding $\left(N^{-}, \psi\right.$ )-equivariant category on $G / B$. We will denote by $\psi_{G / B}$ its only irreducible, i.e., the clean extension of the $\left(N^{-}, \psi\right)$-character perverse sheaf on $N^{-} \cdot 1_{G / B}$.

For $\mathcal{S} \in \mathrm{D}\left(\mathrm{Gr}_{G}\right)^{I}$ we have:

$$
\psi_{N^{-}} \stackrel{!}{\star} \mathcal{S} \simeq \psi_{G / B} \stackrel{!}{\star} \mathcal{S} \text { and } \psi_{N^{-}}{ }^{*} \mathcal{S} \simeq \psi_{G / B} \stackrel{*}{\star} \mathcal{S},
$$

\footnotetext{
${ }^{5} \mathrm{~A}$ more efficient proof of this fact is given in $[\mathrm{BBM}]$
} 
but the map

$$
\psi_{G / B} \stackrel{!}{\star} \mathcal{S} \rightarrow \psi_{G / B} \stackrel{*}{\star} \mathcal{S}
$$

is an isomorphism, since the convolution map $\pi_{I}: G[[t]] \underset{I}{\times} \mathrm{Gr}_{G} \rightarrow \mathrm{Gr}_{G}$ is proper.

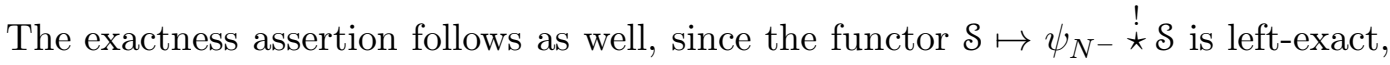
and $\mathcal{S} \mapsto \psi_{N^{-}}{ }^{*} \mathcal{S}$ is right-exact.

Henceforth, we will denote the functor

$$
\left.\left.\operatorname{Av}_{!, I^{-}, \psi}[-\operatorname{dim}(\mathfrak{n})]\right|_{\mathrm{D}\left(\operatorname{Gr}_{G}\right)^{I^{0}}} \simeq \operatorname{Av}_{*, I^{-}, \psi}[\operatorname{dim}(\mathfrak{n})]\right|_{\mathrm{D}\left(\operatorname{Gr}_{G}\right)^{I^{0}}}
$$

simply by $\mathrm{Av}_{I^{-}, \psi}$.

2.2.6. Partial integrability. We say that an object of $\operatorname{Perv}\left(\mathrm{Gr}_{G}\right)^{I^{0}}$ (resp., $\operatorname{Perv}\left(\mathrm{Gr}_{G}\right)^{I}$, $\left.\operatorname{Perv}\left(\mathrm{Fl}_{G}\right)^{I^{0}}, \operatorname{Perv}\left(\mathrm{Fl}_{G}\right)^{I}\right)$ is partially integrable if it admits a filtration, such that each subquotient is equivariant with respect to some parahoric, contained in $G[[t]]$, and strictly containing $I$. (The latter condition is equivalent to demanding that this subquotient is equivariant with respect to some subminimal parabolic $P_{\imath} \subset G \subset G[[t]]$.) Let us denote the resulting Serre subcategories by ${ }^{P I} \operatorname{Perv}\left(\mathrm{Gr}_{G}\right)^{I^{0}}$ (resp., ${ }^{P I} \operatorname{Perv}\left(\mathrm{Gr}_{G}\right)^{I}$, $\left.{ }^{P I} \operatorname{Perv}\left(\mathrm{Fl}_{G}\right)^{I^{0}},{ }^{P I} \operatorname{Perv}\left(\mathrm{Fl}_{G}\right)^{I}\right)$.

Note that an irreducible $\mathrm{IC}_{w \cdot \check{\lambda}, \mathrm{Gr}_{G}} \in \operatorname{Perv}\left(\mathrm{Gr}_{G}\right)^{I^{0}}$ is non-partially integrable if and only if $w=w_{0}$. Similarly, $\mathrm{IC}_{w, G / B} \in \operatorname{Perv}(G / B)$ is partially integrable unless $w=1$.

Let ${ }^{f} \operatorname{Perv}\left(\mathrm{Gr}_{G}\right)^{I^{0}}$ (resp., $\left.{ }^{f} \operatorname{Perv}\left(\mathrm{Gr}_{G}\right)^{I},{ }^{f} \operatorname{Perv}\left(\mathrm{Fl}_{G}\right)^{I^{0}},{ }^{f} \operatorname{Perv}\left(\mathrm{Fl}_{G}\right)^{I}\right)$ be the resulting quotient abelian category of $\operatorname{Perv}\left(\mathrm{Gr}_{G}\right)^{I^{0}}$ (resp., $\left.\operatorname{Perv}\left(\mathrm{Gr}_{G}\right)^{I}, \operatorname{Perv}\left(\mathrm{Fl}_{G}\right)^{I^{0}}, \operatorname{Perv}\left(\mathrm{Fl}_{G}\right)^{I}\right)$, and let ${ }^{f} \mathrm{D}\left(\mathrm{Gr}_{G}\right)^{I^{0}}$ (resp., ${ }^{f} \mathrm{D}\left(\mathrm{Gr}_{G}\right)^{I},{ }^{f} \mathrm{D}\left(\mathrm{Fl}_{G}\right)^{I^{0}},{ }^{f} \mathrm{D}\left(\mathrm{Fl}_{G}\right)^{I}$ ) be the corresponding quotient triangulated category.

The convolution functor descends to functors

$$
{ }^{f} \mathrm{D}\left(\mathrm{Fl}_{G}\right)^{I^{0}} \times \mathrm{D}\left(\mathrm{Gr}_{G}\right)^{I} \rightarrow{ }^{f} \mathrm{D}\left(\mathrm{Gr}_{G}\right){ }^{I^{0}} \text { and }{ }^{f} \mathrm{D}\left(\mathrm{Fl}_{G}\right)^{I} \times \mathrm{D}\left(\mathrm{Gr}_{G}\right)^{I} \rightarrow{ }^{f} \mathrm{D}\left(\mathrm{Gr}_{G}\right)^{I} .
$$

Proposition 2.2.7. The functor

$$
\operatorname{Av}_{I^{-}, \psi}: \operatorname{Perv}\left(\operatorname{Gr}_{G}\right)^{I^{0}} \rightarrow \operatorname{Perv}\left(\operatorname{Gr}_{G}\right)^{I^{-}, \psi}
$$

factors through ${ }^{f} \operatorname{Perv}\left(\mathrm{Gr}_{G}\right)^{I^{0}}$, and the resulting functor ${ }^{f} \operatorname{Perv}\left(\mathrm{Gr}_{G}\right)^{I^{0}} \rightarrow \operatorname{Perv}\left(\mathrm{Gr}_{G}\right)^{I^{-}, \psi}$ is exact and faithful.

Proof. The fact that $\mathrm{Av}_{I^{-}, \psi}$ annihilates all partially integrable objects follows from the observation that the direct image of $\psi_{G / B}$ to any partial flag variety $G / P_{\imath}$ is zero.

The fact that ${ }^{f} \operatorname{Perv}\left(\mathrm{Gr}_{G}\right)^{I^{0}} \rightarrow \operatorname{Perv}\left(\mathrm{Gr}_{G}\right)^{I^{-}, \psi}$ is exact follows from the exactness statement of Proposition 2.2.5. To show that it is faithful, it is enough to prove the corresponding fact for ${ }^{f} \operatorname{Perv}\left(\mathrm{Gr}_{G}\right)^{I}$. We argue as follows:

Let $\mathrm{Av}_{!, I^{0}}: \mathrm{D}\left(\mathrm{Gr}_{G}\right) \rightarrow \mathrm{D}\left(\mathrm{Gr}_{G}\right)^{I^{0}}$ (resp., $\mathrm{D}(G / B) \rightarrow \mathrm{D}(G / B)^{N}$ ) be the functor, left adjoint to the tautological embedding. Let us denote by $\Xi$ the object

$$
\Xi:=\operatorname{Av}_{!, I^{0}}[-\operatorname{dim}(\mathfrak{n})]\left(\psi_{G / B}\right) \in \mathrm{D}(G / B)^{N} .
$$


We have, tautologically:

Lemma 2.2.8. The composition

$$
\operatorname{Perv}\left(\mathrm{Gr}_{G}\right)^{I^{I}} \stackrel{\mathrm{Av}_{I^{-},}, \psi}{\longrightarrow} \operatorname{Perv}\left(\mathrm{Gr}_{G}\right)^{I^{-}, \psi} \rightarrow \operatorname{Perv}\left(\mathrm{Gr}_{G}\right) \stackrel{\operatorname{Av}_{!, I^{0}}[-\operatorname{dim}(\mathfrak{n})]}{\longrightarrow} \operatorname{Perv}\left(\mathrm{Gr}_{G}\right)^{I^{0}}
$$

is isomorphic to the convolution functor

$$
\mathcal{F} \mapsto \Xi \star \mathcal{F} .
$$

It is known that $\Xi$ is the longest indecomposable projective in $\operatorname{Perv}(G / B)^{N}$, and it admits two filtrations: one whose subquotients are the standard objects $j_{!}, w, w \in W$, and another, whose subquotients are the costandard objects $j_{*, w}$.

Note, however, that the arrows $j_{*, w} \rightarrow j_{*, 1}$ and $j_{!, 1} \rightarrow j_{!, w}$ become isomorphisms on ${ }^{f} \operatorname{Perv}(G / B)^{B} \subset{ }^{f} \operatorname{Perv}\left(\mathrm{Fl}_{G}\right)^{I}$. Hence, the image of $\Xi$ in ${ }^{f} \operatorname{Perv}(G / B)^{N}$ is isomorphic to the extension of $|W|$-many copies of $\delta_{1, G / B}$. Hence, the convolution with $\Xi$, viewed as a functor ${ }^{f} \operatorname{Perv}\left(\mathrm{Gr}_{G}\right)^{I} \rightarrow{ }^{f} \operatorname{Perv}\left(\mathrm{Gr}_{G}\right)^{I^{0}}$, is faithful.

Remark. One can strengthen Proposition 2.2.7 and prove the following more precise assertion:

Let $h^{0}$ be the algebra of functions on the scheme-theoretic preimage of 0 under $\mathfrak{h}^{*} \rightarrow \mathfrak{h}^{*} / W$. It is known that $h_{0}$ is isomorphic to the algebra of endomorphisms of $\Xi$.

For an abelian category $\mathcal{C}$ we will denote by $\mathcal{C} \otimes h^{0}$ the category of objects of $\mathcal{C}$, endowed with an action of $h^{0}$.

Then the category ${ }^{f} \operatorname{Perv}\left(\operatorname{Gr}_{G}\right)^{I^{0}}$ is equivalent to $\operatorname{Perv}\left(\mathrm{Gr}_{G}\right)^{I^{-}, \psi} \otimes h^{0} \simeq \mathrm{Sph}_{G} \otimes h^{0}$.

\subsection{Cosocles of costandard objects.}

2.3.1. In this subsection we will prove the following assertion:

\section{Proposition 2.3.2.}

(1) For a regular dominant element $\check{\lambda} \in \check{\Lambda}$, the cosocle of $\mathcal{W}^{*, \check{\lambda}} \in \operatorname{Perv}\left(\operatorname{Gr}_{G}\right)^{I}$ is simple and is isomorphic to $\mathrm{IC}_{w_{0} \cdot \check{\lambda}, \mathrm{Gr}_{G}}$.

(2) The kernel of $\mathcal{W}^{*, \check{\lambda}} \rightarrow \mathrm{IC}_{w_{0} \cdot \check{\lambda}, \mathrm{Gr}_{G}}$ is partially integrable.

Proof. First, we claim that if we have a surjection from $\mathcal{W}^{*, \grave{\lambda}}$ to an irreducible $\mathcal{S}$, then this $\mathcal{S}$ must be non-partially integrable. Suppose the contrary, and let $\imath \in \mathcal{J}$ be such that $\mathcal{S}$ is equivariant with respect to the corresponding sub-minimal parahoric. Then the convolution $j_{!}, s_{\imath} \star \mathcal{S}$ lives in the cohomological degree +1 . However, $j_{s_{\imath}, !} \star \mathcal{W}^{*, \check{\lambda}} \simeq \mathcal{W}^{*, s_{2} \cdot \lambda}$ is still perverse. Hence, $\operatorname{Hom}_{\mathrm{D}\left(\mathrm{Gr}_{G}\right)^{I}}\left(j_{s_{\imath}, !} \star \mathcal{W}^{*, \check{\lambda}}, j_{!, s_{\imath}} \star \mathcal{S}\right)=0$, which is a contradiction, since the convolution with $j_{s_{\imath}, !}$ is an auto-equivalence of $\mathrm{D}\left(\mathrm{Gr}_{G}\right)^{I}$.

To finish the proof of the proposition, it suffices to show that $\mathrm{IC}_{w_{0} \cdot \check{\lambda}, \mathrm{Gr}_{G}}$ is the only non-partially integrable irreducible that appears in the Jordan-Hölder series of $\mathcal{W}^{*, \tilde{\lambda}}$. Since the natural map

$$
\mathcal{W}^{*, \check{\lambda}} \simeq j_{*, \lambda} \star \delta_{1, \operatorname{Gr}_{G}} \rightarrow j_{!, w_{0}} \star j_{*, \lambda} \star \delta_{1, \mathrm{Gr}_{G}} \simeq \mathcal{W}^{*, w_{0} \cdot \check{\lambda}}
$$


becomes an isomorphism in ${ }^{f} \operatorname{Perv}\left(\mathrm{Gr}_{G}\right)^{I}$, by Proposition 2.2.7, it suffices to show that the map

$$
\operatorname{Av}_{I^{-}, \psi}\left(\mathcal{W}^{*, w_{0} \cdot \check{\lambda}}\right) \rightarrow \operatorname{Av}_{I^{-}, \psi}\left(\mathrm{IC}^{w_{0} \cdot \check{\lambda}, \operatorname{Gr}_{G}}\right)
$$

is an isomorphism.

By Proposition 2.2.5, it would be sufficient to show that $\operatorname{Av}_{I^{-}, \psi}\left(\mathcal{W}^{*, w_{0} \cdot \check{\lambda}}\right)$ is an irreducible object of $\operatorname{Perv}\left(\mathrm{Gr}_{G}\right)^{I^{-}, \psi}$. However, evidently,

$$
\operatorname{Av}_{*, I^{-}, \psi}\left(\mathcal{W}^{*, w_{0} \cdot \check{\lambda}}\right)[\operatorname{dim}(\mathfrak{n})]
$$

is the ${ }^{*}$-extension of the corresponding character sheaf on $I^{-} \cdot \check{\lambda}$. Hence, we are done by Theorem $2.2 .2(2)$.

\subsubsection{We will now prove the following:}

Proposition 2.3.4. If $\check{\lambda} \in \check{\Lambda}^{+}$is large, the object $\mathcal{W}^{*, w_{0} \cdot \check{\lambda}}$ admits $\mathrm{IC}_{\check{\lambda}-2 \check{\rho}, \operatorname{Gr}_{G}}$ as a quotient.

The rest of this subsection is devoted to the proof of this result. Let $A v_{!, G[[t]] / I}$ be the functor Perv $\left(\mathrm{Gr}_{G}\right)^{I} \rightarrow \operatorname{Perv}\left(\mathrm{Gr}_{G}\right)^{G[t]]} \simeq \mathrm{Sph}_{G}$ left adjoint to the forgetful functor $\operatorname{Perv}\left(\mathrm{Gr}_{G}\right)^{G[[t]]} \rightarrow \operatorname{Perv}\left(\mathrm{Gr}_{G}\right)^{I}$. Note that since $G[[t]] / I=G / B$ is compact, the corresponding right adjoint $\mathrm{Av}_{*, G[[t]] / I}$ is isomorphic to $\mathrm{Av}_{!, G[[t]] / I}[2 \operatorname{dim}(\mathfrak{n})]$.

For a regular $\check{\lambda} \in \check{\Lambda}^{+}$, let us denote by $\mathrm{emb}_{!, \mathrm{Gr}_{G}}^{\check{\lambda}}, \mathrm{emb}_{*, \mathrm{Gr}_{G}}^{\check{\lambda}}$ the natural functors $\mathrm{D}(G / B) \rightarrow \mathrm{D}\left(\mathrm{Gr}_{G}\right)$, along with its $I$ - and $I^{0}$-equivariant versions. Evidently, these functors commute with $\mathrm{Av}_{!, G[[t]] / I}$ in the natural sense. Therefore,

$$
\operatorname{Av}_{!, G[[t]] / I}\left(\mathcal{W}^{*, w_{0} \cdot \check{\lambda}}\right) \simeq \operatorname{emb}_{*, \mathrm{Gr}_{G}}^{\check{\lambda}}\left(\underline{\mathbb{C}}_{G / B}[2 \operatorname{dim}(\mathfrak{n})]\right) .
$$

In particular, we obtain that the object $\mathrm{emb}_{*, \mathrm{Gr}_{G}}^{\check{\lambda}}\left(\mathrm{IC}_{G / B}\right)$ lives in the cohomological

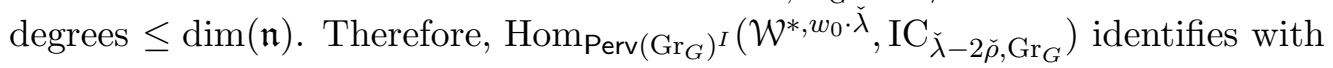

$$
\operatorname{Hom}_{\operatorname{Perv}\left(\operatorname{Gr}_{G}\right)^{G[[t]]}}\left(h^{\operatorname{dim}(\mathfrak{n})}\left(\operatorname{emb}_{*, \operatorname{Gr}_{G}}^{\check{\check{\lambda}}}\left(\mathrm{IC}_{G / B}\right)\right), \mathrm{IC}_{\check{\lambda}-2 \check{\rho}, \operatorname{Gr}_{G}}\right) .
$$

Thus, we have to show that the top=dim(n)-degree cohomology of $\mathrm{emb}_{*, \mathrm{Gr}_{G}}^{\check{\lambda}}\left(\mathrm{IC}_{G / B}\right)$ has a quotient (or, which in this case is the same, a direct summand) isomorphic to $\mathrm{IC}_{\check{\lambda}-2 \check{\rho}, \mathrm{Gr}_{G}}$. Set $\check{\mu}=\check{\lambda}-2 \check{\rho}$.

Consider the cohomology

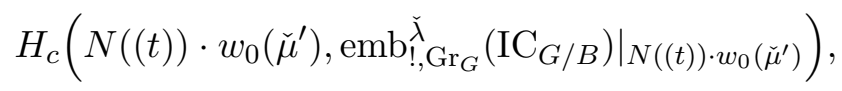

where, as usual, we regard $N((t)) \cdot w_{0}(\check{\mu})$ as a sub-indscheme in $\mathrm{Gr}_{G}$. By [MV] (and duality) it would suffice to show that the above cohomology in degree $-\operatorname{dim}(\mathfrak{n})-\left\langle 2 \rho, \check{\mu}^{\prime}\right\rangle$ is 1-dimensional if $\check{\mu}^{\prime}=\check{\mu}$, and is 0 for $\check{\mu}<\check{\mu}^{\prime} \leq \check{\lambda}$.

By base change, the above cohomology can be rewritten as

$$
H_{c}^{\left\langle 2 \rho, \check{\lambda}-\check{\mu}^{\prime}\right\rangle-\operatorname{dim}(\mathfrak{n})}\left(\left(N((t)) \cdot w_{0}\left(\check{\mu}^{\prime}\right)\right) \cap \mathrm{Gr}_{G}^{\check{\lambda}}, \mathbb{C}\right) .
$$


MODULES OVER THE SMALL QUANTUM GROUP AND SEMI-INFINITE FLAG MANIFOLD 27

Since $\check{\lambda}$ was assumed large, the intersection $\left(N((t)) \cdot w_{0}\left(\check{\mu}^{\prime}\right)\right) \cap \mathrm{Gr}_{G}^{\check{\lambda}}$ equals $\left.\left.\left(N((t)) \cdot w_{0}\left(\check{\mu}^{\prime}\right)\right) \cap\left(N^{-}((t)) \cdot w_{0}(\check{\lambda})\right)\right) \simeq\left(N((t)) \cdot 1_{\mathrm{Gr}_{G}}\right) \cap\left(N^{-}((t)) \cdot w_{0}\left(\check{\lambda}-\check{\mu}^{\prime}\right)\right)\right)$.

Hence, our assertion follows from Corollary 4.3.8.

Corollary 2.3.5. For $\check{\lambda}$ large the map $\mathcal{W}^{*, \check{\lambda}} \rightarrow \mathrm{IC}_{w_{0} \cdot \check{\lambda}, \mathrm{Gr}_{G}}$ lifts to a map $\mathcal{W}^{*, \check{\lambda}} \rightarrow$ $\mathcal{W}^{!}, w_{0} \cdot \check{\rho}^{\prime} \star \mathrm{IC}_{\check{\lambda}-\check{\rho}^{\prime}, \mathrm{Gr}_{G}}$.

Proof. The existence of the map in question is equivalent, by adjunction, to the exis-

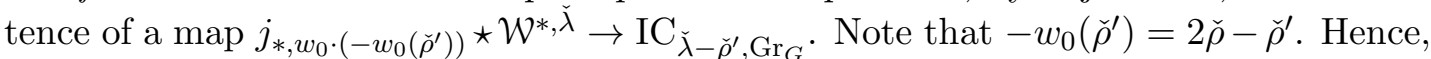
the assertion follows from the above proposition, since:

$$
j_{*, w_{0} \cdot\left(2 \check{\rho}-\check{\rho}^{\prime}\right)} \star \mathcal{W}^{*, \check{\lambda}} \simeq j_{*, w_{0} \cdot\left(2 \check{\rho}-\check{\rho}^{\prime}\right)} \star j_{*, \check{\lambda}} \star \delta_{1, \mathrm{Gr}_{G}} \simeq j_{*, w_{0} \cdot\left(\check{\lambda}+2 \check{\rho}-\check{\rho}^{\prime}\right)} \star \delta_{1, \mathrm{Gr}_{G}} \simeq \mathcal{W}^{*, \check{\lambda}+2 \check{\rho}-\check{\rho}^{\prime}}
$$

\section{A STUdy OF BABy VERma AND CO-VERma MOdules}

\subsection{Baby co-Verma modules via $\mathbf{U}_{\ell}$.}

3.1.1. Let $\dot{\mathbf{M}}^{\lambda}$ be the object of $\dot{H}$ ecke $\left(\mathbf{U}_{\ell}, \check{G}\right)$, corresponding to $\dot{M}^{\lambda}$. Our present goal is to describe it explicitly. First, we will describe $\dot{\mathbf{M}}^{\lambda}$ as an object of $\mathbf{U}_{\ell}-\overline{\bmod }$. By definition, $\mathbf{M}^{\lambda}=\underset{\check{\mu} \in \check{\Lambda}}{\oplus} \mathbf{M}_{\check{\mu}}^{\lambda}$, where each $\mathbf{M}_{\check{\mu}}^{\lambda}$ is given by

$$
\operatorname{Ind}_{\mathfrak{u}_{\ell}}^{\mathbf{U}_{\ell}}\left(\mathbf{C}^{-\check{\mu}} \otimes \dot{M}^{\lambda}\right) \simeq \operatorname{Ind}_{\mathfrak{u}_{\ell}}^{\mathbf{U}_{\ell}}\left(\dot{M}^{\lambda-\phi_{\ell}(\check{\mu})}\right)
$$

Hence, it sufficient to describe the modules of the form $\operatorname{Ind}_{\mathfrak{u}_{\ell}}^{\mathbf{U}_{\ell}}\left(\dot{M}^{\lambda}\right)$.

By construction,

$$
\operatorname{Ind}_{\mathfrak{u}_{\ell}}^{\mathbf{U}_{\ell}}\left(\dot{M}^{\lambda}\right) \simeq \operatorname{Ind}_{\mathfrak{b}_{\ell}^{-}}^{\mathbf{U}_{\ell}}\left(\mathbf{C}^{\lambda}\right)
$$

which, in turn, is isomorphic to

$$
\operatorname{Ind}_{\mathbf{B}_{\ell}^{-}}^{\mathbf{U}_{\ell}}\left(\operatorname{Ind}_{\dot{b}_{\ell}^{-}}^{\mathbf{B}_{\ell}^{-}}\left(\mathbf{C}^{\lambda}\right)\right) \simeq \operatorname{Ind}_{\mathbf{B}_{\ell}^{-}}^{\mathbf{U}_{\ell}}\left(\mathbf{C}^{\lambda} \otimes \operatorname{Ind}_{\dot{b}_{\ell}^{-}}^{\mathbf{B}_{\ell}^{-}}(\mathbb{C})\right) .
$$

By Proposition 1.1.14, $\operatorname{Ind}_{\dot{b}_{\ell}^{-}}^{\mathbf{B}_{\ell}^{-}}(\mathbb{C}) \simeq \operatorname{Fr}_{B^{-}}\left(\mathcal{O}_{\check{B}^{-} / \check{T}}\right)$.

Proposition-Construction 3.1.2. As a $\check{B}^{-}$-module, $\mathcal{O}_{\check{B}^{-}} / \check{T}$ is isomorphic to the direct limit

$$
\underset{\check{\lambda} \in \check{\Lambda}^{+}}{\lim } \operatorname{Res}_{\check{B}^{-}}^{\check{G}}\left(\left(V^{\check{\lambda}}\right)^{*}\right) \otimes \mathfrak{l}^{\check{\lambda}},
$$

$\mathfrak{l}^{\check{\lambda}}$ denotes highest weight line of $V^{\check{\lambda}}$, regarded as a 1 -dimensional representation of $\check{T}$ (and, hence, also of $\check{B}^{-}$). 
Proof. By adjunction, to specify a map of $\check{B}^{-}$-modules

$$
\operatorname{Res}_{\check{B}^{-}}^{\check{\check{C}}}\left(\left(V^{\check{\lambda}}\right)^{*}\right) \otimes \mathfrak{l}^{\check{\lambda}} \rightarrow \mathcal{O}_{\check{B}^{-}} / \check{T}
$$

is equivalent to giving a map $\left(\underline{V}^{\check{\lambda}}\right)^{*} \rightarrow\left(\mathfrak{l}^{\check{\lambda}}\right)^{*}$, compatible with the $T$-action. The latter corresponds to the natural embedding of $\mathfrak{l}^{\check{\lambda}}$ into $\underline{V}^{\check{\lambda}}$.

To define the inductive system, we choose a compatible system of isomorphisms $\mathfrak{l}^{\check{\lambda}} \otimes \mathfrak{l}^{\check{\mu}} \simeq \mathfrak{l}^{\check{\lambda}+\check{\mu}}$. Such a system fixes as the maps $V^{\check{\lambda}} \otimes V^{\check{\mu}} \rightarrow V^{\check{\lambda}+\check{\mu}}$ (which are otherwise defined up to a scalar).

Suppose that $\check{\mu} \in \check{\Lambda}^{+}$is another dominant weight of $\check{G}$. We define the map

$$
\operatorname{Res}_{\check{B}^{-}}^{\check{G}}\left(\left(V^{\check{\lambda}}\right)^{*}\right) \otimes \mathfrak{l}^{\check{\lambda}} \rightarrow \operatorname{Res}_{\check{B}^{-}}^{\check{\check{G}}}\left(\left(V^{\check{\lambda}+\check{\mu}}\right)^{*}\right) \otimes \mathfrak{l}^{\check{\lambda}+\check{\mu}}
$$

as the composition

(10) $\operatorname{Res}_{\check{B}^{-}}^{\check{G}}\left(\left(V^{\check{\lambda}}\right)^{*}\right) \otimes \mathfrak{l}^{\check{\lambda}} \rightarrow \operatorname{Res}_{\check{B}^{-}}^{\check{G}}\left(\left(V^{\check{\lambda}} \otimes V^{\check{\mu}}\right)^{*}\right) \otimes \mathfrak{l}^{\check{\mu}} \otimes \mathfrak{l}^{\check{\lambda}} \rightarrow \operatorname{Res}_{\check{B}^{-}}^{\check{G}}\left(\left(V^{\check{\lambda}+\check{\mu}}\right)^{*}\right) \otimes \mathfrak{l}^{\check{\lambda}+\check{\mu}}$,

where the first arrow comes from the map of $\check{B}^{-}$-modules $\left(\check{\mathfrak{l}}^{\check{\mu}}\right)^{*} \rightarrow \operatorname{Res}_{\check{B}^{-}}^{\check{G}}\left(\left(V^{\check{\mu}}\right)^{*}\right)$, and the second arrow comes from the map $\left(V^{\check{\lambda}} \otimes V^{\check{\mu}}\right)^{*} \rightarrow\left(V^{\check{\lambda}+\check{\mu}}\right)^{*}$. These maps define the inductive system stated in the Proposition-Construction.

By construction, the map of (10) is compatible with the maps of (9) for $\check{\lambda}$ and $\check{\mu}$. Hence, the resulting inductive limit maps to $\mathcal{O}_{\check{B}^{-}} / \check{T}$. The fact that this map is an isomorphism is an easy verification.

3.1.3. ¿From the above Proposition we obtain the following description of $\mathbf{M}_{\tilde{\nu}}^{\lambda}$ :

Corollary 3.1.4. Choose a trivialization of the $\check{T}$-torsor given by $\left\{\mathfrak{l}^{\grave{\lambda}}\right\}$. Then

$$
\mathbf{M}_{\check{\nu}}^{\lambda} \simeq \underset{\check{\lambda} \in \check{\Lambda}^{+}}{\lim _{\vec{m}}} \operatorname{Fr}\left(\left(V^{\check{\lambda}}\right)^{*}\right) \otimes \mathbf{W}^{\lambda+\phi_{\ell}(\check{\lambda}-\check{\nu})},
$$

where the maps in the inductive system are given by

$$
\begin{aligned}
& \operatorname{Fr}\left(V^{\check{\lambda}}\right)^{*} \otimes \mathbf{W}^{\lambda+\phi_{\ell}(\check{\lambda}-\check{\nu})} \rightarrow \operatorname{Fr}\left(V^{\check{\lambda}}\right)^{*} \otimes \operatorname{Fr}\left(V^{\check{\mu}}\right)^{*} \otimes \mathbf{W}^{\lambda+\phi_{\ell}(\check{\lambda}+\check{\mu}-\check{\nu})} \rightarrow \\
& \operatorname{Fr}\left(V^{\check{\lambda}+\check{\mu}}\right)^{*} \otimes \mathbf{W}^{\lambda+\phi_{\ell}(\check{\lambda}+\check{\mu}-\check{\nu})},
\end{aligned}
$$

where the first arrow comes from the canonical map

$$
\operatorname{Fr}\left(V^{\check{\mu}}\right) \otimes \mathbf{W}^{\lambda+\phi_{\ell}(\check{\lambda}-\check{\nu})} \rightarrow \mathbf{W}^{\lambda+\phi_{\ell}(\check{\lambda}+\check{\mu}-\check{\nu})} .
$$

3.1.5. Hecke property. Let us now describe how the Hecke isomorphisms

$$
\operatorname{Fr}(V) \otimes \mathbf{M}_{\check{\mu}}^{\lambda} \rightarrow \underset{\check{\nu} \in \check{\Lambda}^{+}}{\oplus} \mathbf{M}_{\check{\mu}-\check{\nu}}^{\lambda} \otimes \underline{V}(\check{\nu})
$$

look like in terms of the identification of Corollary 3.1.4.

For a coweight $\check{\lambda} \in \check{\Lambda}^{+}$, large with respect to the weights of $V$, we have a canonical isomorphism of $\check{G}$-modules

$$
V \otimes V^{\check{\lambda}} \simeq \underset{\check{\nu}}{\oplus} V^{\check{\lambda}+\check{\nu}} \otimes \underline{V}(\check{\nu})
$$


Hence, we obtain a map of $\check{B}^{-}$-modules

$$
V \rightarrow \underset{\check{\nu}}{\oplus} V^{\check{\lambda}+\check{\nu}} \otimes\left(V^{\check{\lambda}}\right)^{*} \otimes \underline{V}(\check{\nu}) \rightarrow\left(V^{\check{\lambda}}\right)^{*} \otimes \mathfrak{l}^{\check{\lambda}+\check{\nu}} \otimes \underline{V}(\check{\nu}) .
$$

Applying the functor $\operatorname{Ind}_{\mathbf{B}_{\ell}^{-}}^{\mathbf{U}_{\ell}}$, for $\lambda \in \Lambda^{+}$, we obtain a map in $\mathbf{U}_{\ell}$-mod:

$$
\operatorname{Fr}(V) \otimes \mathbf{W}^{\lambda} \rightarrow \underset{\check{\nu}}{\oplus} \operatorname{Fr}\left(\left(V^{\check{\lambda}}\right)^{*}\right) \otimes \mathbf{W}^{\lambda+\phi_{\ell}(\check{\lambda}+\check{\nu})} \otimes \underline{V}(\check{\nu}) .
$$

Proposition 3.1.6. The Hecke morphisms for $\dot{\mathbf{M}}^{\lambda}$ are equal in terms of the inductive system to

$$
\begin{aligned}
& \operatorname{Fr}(V) \otimes \operatorname{Fr}\left(\left(V^{\check{\lambda}^{\prime}}\right)^{*}\right) \otimes \mathbf{W}^{\lambda+\phi_{\ell}\left(\check{\lambda}^{\prime}-\check{\mu}\right)} \rightarrow \\
& \underset{\check{\nu}}{\oplus} \operatorname{Fr}\left(\left(V^{\check{\lambda}^{\prime}}\right)^{*} \otimes\left(V^{\check{\lambda}}\right)^{*}\right) \otimes \mathbf{W}^{\lambda+\phi_{\ell}\left(\check{\lambda}+\check{\lambda}^{\prime}-\check{\mu}+\check{\nu}\right)} \otimes \underline{V}(\check{\nu}) \rightarrow \\
& \underset{\check{\nu}}{\oplus} \operatorname{Fr}\left(\left(V^{\check{\lambda}+\check{\lambda}^{\prime}}\right)^{*}\right) \otimes \mathbf{W}^{\lambda+\phi_{\ell}\left(\check{\lambda}+\check{\lambda}^{\prime}-\check{\mu}+\check{\nu}\right)} \otimes \underline{V}(\check{\nu}) .
\end{aligned}
$$

Proof. By the construction of the isomorphism in Corollary 3.1.4, it suffices to show that the isomorphism

$$
\operatorname{Res}_{\check{B}^{-}}^{\check{G}}(V) \otimes \mathcal{O}_{\check{B}^{-}} / \check{T} \rightarrow \underset{\nu}{\oplus} \mathcal{O}_{\check{B}^{-} / \check{T}} \otimes \underline{V}(\check{\nu})
$$

looks in terms of the identification given by Proposition-Construction 3.1.2 as a system of morphisms

$$
\begin{aligned}
& \left.\operatorname{Res}_{\check{B}^{-}}^{\check{G}}(V) \otimes\left(V^{\check{\lambda}^{\prime}}\right)^{*}\right) \otimes \mathfrak{l}^{\check{\lambda}^{\prime}} \rightarrow \underset{\check{\nu}}{\oplus} \operatorname{Res}_{\check{B}}^{\check{G}}\left(\left(V^{\check{\lambda}^{\prime}}\right)^{*} \otimes\left(V^{\check{\lambda}}\right)^{*}\right) \otimes \mathfrak{l}^{\check{\lambda}+\check{\lambda}^{\prime}+\check{\nu}} \otimes \underline{V}(\check{\nu}) \rightarrow \\
& \rightarrow \underset{\check{\nu}}{\oplus} \operatorname{Res}_{\check{B}^{-}}^{\check{G}}\left(\left(V^{\check{\lambda}+\check{\lambda}^{\prime}}\right)^{*}\right) \otimes \mathfrak{l}^{\check{\lambda}+\check{\lambda}^{\prime}+\check{\nu}} \otimes \underline{V}(\check{\nu}) .
\end{aligned}
$$

The latter is a straightforward verification.

3.1.7. Baby co-Verma as a quotient. Let us briefly discuss another realization of $\dot{M}^{\lambda}$ (or, equivalently, $\dot{\mathbf{M}}^{\lambda}$ ) in terms of the big quantum group.

For an element $\check{\mu} \in \check{\Lambda}^{*}$, let $\stackrel{\circ}{\mathscr{}}^{\check{\mu}}$ be the hyperplane in $\underline{V}^{\check{\mu}}$ orthogonal to $\mathfrak{l}^{-\check{\mu}} \subset\left(\underline{V}^{\check{\mu}}\right)^{*}$. This subspace is preserved by $\check{B}^{-}$, and in particular, it admits a well-defined weight decomposition with respect to $\check{T}$.

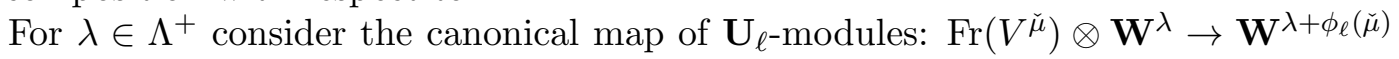
After the restriction to $\dot{\mathfrak{u}}_{\ell}$, it gives rise to a map

$$
\underset{\check{\nu}}{\oplus} \mathbf{C}^{\check{\nu}} \otimes \operatorname{Res}_{\mathfrak{u}_{\ell}}^{\mathbf{U}_{\ell}}\left(\mathbf{W}^{\lambda}\right) \otimes \underline{V}^{\check{\mu}}(\check{\nu}) \rightarrow \operatorname{Res}_{\mathfrak{u}_{\ell}}^{\mathbf{U}_{\ell}}\left(\mathbf{W}^{\lambda+\phi_{\ell}(\check{\mu})}\right) .
$$

For $\check{\lambda} \in \check{\Lambda}^{+}$consider the canonical map $\operatorname{Res}_{\mathfrak{u}_{\ell}}^{\mathbf{U}_{\ell}}\left(\mathbf{W}^{\lambda+\phi_{\ell}(\mu)}\right) \rightarrow \dot{M}^{\lambda+\phi_{\ell}(\mu)}$.

Proposition 3.1.8. The composition

$$
\underset{\check{\nu}}{\oplus} \mathbf{C}^{\check{\nu}} \otimes \operatorname{Res}_{\dot{\mathfrak{u}}_{\ell}}^{\mathbf{U}_{\ell}}\left(\mathbf{W}^{\lambda}\right) \otimes \stackrel{\circ}{V}^{\check{\mu}}(\check{\nu}) \rightarrow \operatorname{Res}_{\mathfrak{u}_{\ell}}^{\mathbf{U}_{\ell}}\left(\mathbf{W}^{\lambda+\phi_{\ell}(\check{\mu})}\right) \rightarrow \dot{M}^{\lambda+\phi_{\ell}(\mu)}
$$


vanishes. For a fixed $\lambda$ and all sufficiently large $\check{\mu}$ the complex

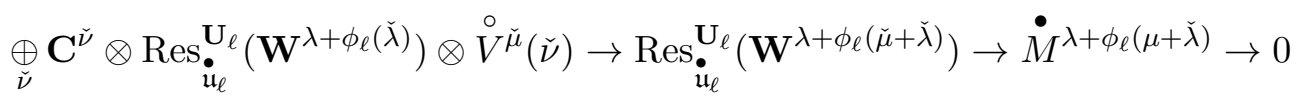

is exact for all sufficiently dominant $\check{\lambda}$.

Proof. The first assertion of the proposition is evident. To prove the second one we proceed as follows. Let $\mathbb{C}_{\check{B}^{-}}$be the sky-scraper coherent sheaf at the point $\check{B}^{-}$in the flag variety $\breve{G} / \check{B}^{-}$. It admits a left resolution of the form

$$
0 \rightarrow \mathcal{P}_{\operatorname{dim}\left(\check{G} / \check{B}^{-}\right)+1} \rightarrow \mathcal{P}_{\operatorname{dim}\left(\check{G} / \check{B}^{-}\right)} \rightarrow \mathcal{P}_{\operatorname{dim}\left(\check{G} / \check{B}^{-}\right)-1} \rightarrow \ldots \rightarrow \mathcal{P}_{1} \rightarrow \mathcal{P}_{0} \rightarrow \mathbb{C}_{\check{B}^{-}} \rightarrow 0
$$

where $\mathcal{P}_{0} \simeq \mathcal{O}_{\check{G} / \check{B}^{-}}$, and the sheaves $\mathcal{P}_{i}$ for $i=1, \ldots, \operatorname{dim}\left(\check{G} / \check{B}^{-}\right)$are isomorphic to $\mathcal{O}\left(-\check{\mu}_{i}\right) \otimes V^{i}$ for $\check{\mu}_{i} \in \check{\Lambda}^{+} ; V^{i}$ are some vector spaces. Moreover, the weight $\check{\mu}_{1}$ may be chosen arbitrarily large; and the vector space $V^{1}$ surjects by construction onto $\stackrel{\circ}{V}^{\check{\mu}_{1}}$.

By pulling back this complex from $\check{G} / \check{B}^{-}$to $\check{G}$, it gives rise to a complex

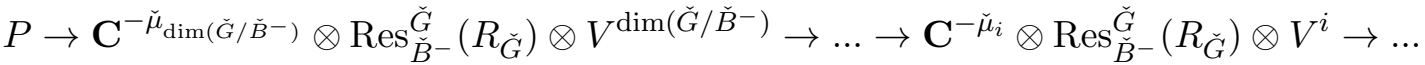

$$
\begin{aligned}
& \ldots \rightarrow \mathbf{C}^{-\check{\mu}_{1}} \otimes \operatorname{Res}_{\check{B}^{-}}^{\check{G}}\left(R_{\check{G}}\right) \otimes V^{1} \rightarrow \operatorname{Res}_{\check{B}^{-}}^{\check{G}}\left(R_{\check{G}}\right) \rightarrow R_{\check{B}^{-}} \rightarrow 0
\end{aligned}
$$

of $\check{B}^{-}$-modules, where $R_{\check{B}^{-}}$denotes the regular representation of $\check{B}^{-}$. By construction, the arising element in $\operatorname{Ext}_{\check{B}^{-}}^{\operatorname{dim}\left(\check{G} / \check{B}^{-}\right)+1}\left(R_{\check{B}^{-}}, P\right)$ vanishes.

Let us tensor this complex with the $\mathbf{B}_{\ell}^{-}$-module $\mathbf{C}^{\lambda+\phi_{\ell}\left(\check{\mu}_{1}+\check{\lambda}\right)}$, where $\check{\lambda}$ is such that all the weights of the form $\lambda+\phi_{\ell}\left(\check{\mu}_{1}+\check{\lambda}-\check{\mu}_{i}\right)$ become dominant. Then,

$$
\begin{aligned}
& \mathrm{R}^{i} \operatorname{Ind}_{\mathbf{B}_{\ell}^{-}}^{\mathbf{U}_{\ell}}\left(\mathbf{C}^{\lambda+\phi_{\ell}\left(\check{\lambda}^{+}+\check{\mu}_{1}-\check{\mu}_{i}\right)} \otimes \operatorname{Fr}_{\check{B}^{-}}\left(\operatorname{Res}_{\check{B}^{-}}^{\check{G}}\left(R_{\breve{G}}\right)\right)\right) \simeq \\
& \simeq \operatorname{Fr}\left(R_{\breve{G}}\right) \otimes \mathrm{R}^{i} \operatorname{Ind}_{\mathbf{B}_{\ell}^{-}}^{\mathbf{U}_{\ell}}\left(\mathbf{C}^{\lambda+\phi_{\ell}\left(\check{\lambda}^{\prime}+\check{\mu}_{1}-\check{\mu}_{i}\right)}\right)=0
\end{aligned}
$$

for $i>0$.

Hence, we obtain that the sequence of $\mathbf{U}_{\ell \text {-modules }}$

$$
\operatorname{Fr}\left(R_{\check{G}}\right) \otimes \mathbf{W}^{\lambda+\phi_{\ell}(\check{\lambda})} \otimes \stackrel{\circ}{\mu^{\mu}} \rightarrow \operatorname{Fr}\left(R_{\check{G}}\right) \otimes \mathbf{W}^{\lambda+\phi_{\ell}\left(\check{\mu}_{1}+\check{\lambda}\right)} \rightarrow \operatorname{Ind}_{\mathbf{B}_{\ell}^{-}}^{\mathbf{U}_{\ell}}\left(\mathbf{C}^{\lambda+\phi_{\ell}\left(\check{\mu}_{1}+\check{\lambda}\right)} \otimes R_{\check{B}^{-}}\right) \rightarrow 0
$$

is exact. However, the above sequence of maps is obtained from (11) for $\check{\mu}=\check{\mu}_{1}$ by applying the functor $\operatorname{Ind}_{\mathfrak{u}_{\ell}}^{\mathbf{U}_{\ell}} \circ \operatorname{Res}_{\mathfrak{u}_{\ell}}^{\mathfrak{u}_{\ell}}$, which is exact and faithful.

3.1.9. The case of twisted baby co-Verma modules. For $w \in W$ let $\mathrm{F}_{w}, w_{\ell}, w_{\breve{G}}$ be as in Sect. 1.1.15. ¿From Sect. 1.2.5 we obtain the following description of the object ${ }^{w} \dot{\mathbf{M}}^{w(\lambda)}$ of the category $\dot{H}$ ecke $\left(\mathbf{U}_{\ell}, \check{G}\right)$, corresponding to ${ }^{w} \dot{M}^{w(\lambda)}$ :

Corollary 3.1.10. As an object of $\mathbf{U}_{\ell}-\overline{\bmod },{ }^{w} \dot{\mathbf{M}}_{\breve{\mu}}^{w(\lambda)}$ is isomorphic to $\dot{\mathbf{M}}_{w(\check{\mu})}^{\lambda}$. The Hecke property morphisms

$$
\operatorname{Fr}(V) \otimes{ }^{w} \dot{\mathbf{M}}_{\breve{\mu}}^{w(\lambda)} \rightarrow \underset{\check{\nu}}{\oplus^{w}} \dot{\mathbf{M}}_{\breve{\mu}}^{w(\lambda)} \otimes \underline{V}(\nu)
$$


are obtained from those of $\dot{\mathbf{M}}_{w(\check{\mu})}^{\lambda}$ by applying the element $w_{\breve{G}}: V(\nu) \rightarrow V(w(\nu))$.

In addition, we have an analogue of Proposition 3.1.8. Let ${ }^{w} \stackrel{\circ}{V}^{\check{\mu}}$ be the subspace of $\underline{V}^{\check{\mu}}$ obtained by translating $\underline{\circ}^{\check{\mu}}$ by means of $w_{\breve{G}}$.

Corollary 3.1.11. We have a complex

$$
\underset{\check{\nu}}{\oplus} \mathbf{C}^{\check{\nu}} \otimes \operatorname{Res}_{\mathfrak{u}_{\ell}}^{\mathbf{U}_{\ell}}\left(\mathbf{W}^{\lambda+\phi_{\ell}(\check{\lambda})}\right) \otimes{ }^{w} \stackrel{\circ}{V^{\check{\mu}}}(\check{\nu}) \rightarrow \operatorname{Res}_{\dot{\mathfrak{u}}_{\ell}}^{\mathbf{U}_{\ell}}\left(\mathbf{W}^{\lambda+\phi_{\ell}(\check{\mu}+\check{\lambda})}\right) \rightarrow{ }^{w} \stackrel{\bullet}{M}^{w\left(\lambda+\phi_{\ell}(\mu+\check{\lambda})\right)} \rightarrow 0,
$$

which is exact when for a fixed $\lambda$, the coweights $\check{\mu}$ and $\check{\lambda}$ are large enough.

3.1.12. The non-graded version. For $\lambda \in \Lambda$ recall that $M^{\lambda}$ denotes the restriction of $\dot{M}^{\lambda}$ to $\mathfrak{u}_{\ell}$ (the small, non-graded quantum group). Let $\mathbf{M}^{\lambda}$ be the corresponding object of Hecke $\left(\mathbf{U}_{\ell}, \check{G}\right)$. From Corollary 3.1.4 and we obtain a description of $\mathbf{M}^{\lambda}$ as an object of $\mathbf{U}_{\ell}$-mod. Namely,

$$
\mathbf{M}^{\lambda} \simeq \underset{\check{\mu}}{\oplus \underset{\bar{\lambda} \in \check{\Lambda}^{+}}{\lim }} \operatorname{Fr}\left(\left(V^{\check{\lambda}}\right)^{*}\right) \otimes \mathbf{W}^{\lambda+\phi_{\ell}(\check{\lambda})+\phi_{\ell}(\check{\mu})} .
$$

The Hecke isomorphisms for $\mathbf{M}^{\lambda}$ are given by disregarding the grading in the isomorphisms for $\dot{\mathbf{M}}^{\lambda}$, given by Proposition 3.1.6.

In addition, we can realize $M^{\lambda}$ as a quotient of modules, restricted from $\mathbf{U}_{\ell}$, using Proposition 3.1.8:

$$
M^{\lambda} \simeq \operatorname{coker}\left(\operatorname{Res}_{\mathfrak{u}_{\ell}}^{\mathbf{U}_{\ell}}\left(\mathbf{W}^{\lambda+\phi_{\ell}(\check{\lambda})}\right) \otimes \stackrel{\circ}{V}^{\check{\mu}} \rightarrow \operatorname{Res}_{\mathfrak{u}_{\ell}}^{\mathbf{U}_{\ell}}\left(\mathbf{W}^{\lambda+\phi_{\ell}(\check{\mu}+\check{\lambda})}\right)\right) .
$$

3.1.13. $\check{G}$-action on baby co-Verma modules. By Sect. 1.2 .5 , to any $\mathbf{g} \in \check{G}$ we can attach a module ${ }^{\mathbf{g}} M^{\lambda} \in \mathfrak{u}_{\ell}$-mod. Explicitly, ${ }^{\mathbf{g}} M^{\lambda}$ corresponds to the object ${ }^{\mathbf{g}} \mathbf{M}^{\lambda} \in$ Hecke $\left(\mathbf{U}_{\ell}, \check{G}\right)$, where the latter is obtained from $\mathbf{M}^{\lambda}$ by modifying the Hecke isomorphism using $\mathbf{g}$ acting on $\underline{V}$ for $V \in \check{G}$-mod. Equivalently, ${ }^{\mathbf{g}} M^{\lambda}$ can be realized as

$$
\operatorname{coker}\left(\operatorname{Res}_{\mathfrak{u}_{\ell}}^{\mathbf{U}_{\ell}}\left(\mathbf{W}^{\lambda+\phi_{\ell}(\check{\lambda})}\right) \otimes \underline{\mathbf{g}}_{\underline{V}^{\check{\mu}}}^{\circ} \rightarrow \operatorname{Res}_{\mathfrak{u}_{\ell}}^{\mathbf{U}_{\ell}}\left(\mathbf{W}^{\lambda+\phi_{\ell}(\check{\mu}+\check{\lambda})}\right)\right),
$$

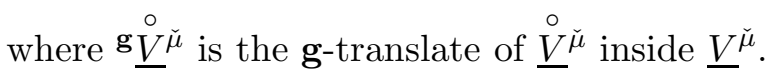

By Sect. 1.2.5 if $\mathbf{g}$ belongs to the normalizer of the torus $\check{T} \subset \check{G}, \mathrm{~g}_{M^{\lambda}}$ is isomorphic to $\operatorname{Res}_{\mathfrak{u}_{\ell}}^{\mathfrak{\mathfrak { u }}_{\ell}}\left({ }^{w} \dot{M}^{w(\lambda)}\right)$ for the corresponding $w \in W$.

Proposition 3.1.14. If $\mathbf{g} \in \check{B}^{-}$, then $\mathbf{g}_{M^{\lambda}}$ is isomorphic to $M^{\lambda}$. For $\lambda=0$ the above condition is "if and only if".

Proof. The description of $\mathbf{g}_{M^{\lambda}}$ given by (14) makes it clear that if $\mathbf{g} \in \check{B}^{-}$, then $\mathrm{g}_{M^{\lambda}} \simeq M^{\lambda}$. To show the inclusion in the opposite direction we argue as follows:

It is easy to see that the subset of elements of $\check{G}$, which stabilize the isomorphism class of $M^{\lambda}$ is a Zariski-closed subgroup of $\check{G}$. Hence, we must show that this subgroup does not contain any parabolic strictly containing $\check{B}^{-}$. Therefore, it is enough to show 
that the none of the modules $\operatorname{Res}_{\mathfrak{u}_{\ell}}^{\dot{\mathfrak{u}}_{\ell}}\left({ }^{w} \dot{M}^{0}\right)$ for $w \neq 1$ is isomorphic to $M^{0}$. This is equivalent to $\dot{M}^{0}$ being non-isomorphic to ${ }^{w} \dot{M}^{\phi_{\ell}(\check{\mu})}$ for $\check{\mu} \in \check{\Lambda}, 1 \neq w \in W$.

Note that the socle of ${ }^{w} \dot{M}^{\phi_{\ell}(\check{\mu})}$ is isomorphic to $\mathbf{C}^{\phi_{\ell}(\check{\mu})}$. Hence, if ${ }^{w} \dot{M}^{\phi_{\ell}(\check{\mu})} \simeq \dot{M}^{0}$, then $\check{\mu}=0$. However, it is clear that ${ }^{w} \dot{M}^{0}$ is non-isomorphic to $\dot{M}^{0}$, because, for example, $-\phi_{\ell}(2 \check{\rho})+2 \rho$, which appears as a weight of $\dot{M}^{0}$, is not among the weights of ${ }^{w} \dot{M}^{0}$.

One can show that the condition of the proposition is in fact "if and only if" for any $\lambda$ belonging to the regular block. This is because, as we shall see later, baby co-Verma modules with the same $w$, but different parameters $\lambda$, can be obtained from one another by (invertible) convolution functors.

\subsection{Baby co-Verma modules via perverse sheaves on the affine Grassman-} nian.

3.2.1. For an element $\tilde{w} \in W_{a f f}$, let $\lambda \in \Lambda$ be the corresponding weight in the regular block. That is, if $\tilde{w}=w \cdot \check{\lambda}$, then $\lambda=\phi_{\ell}(\check{\lambda})+w^{-1}(\rho)-\rho$.

Let $\dot{\mathcal{M}}^{\tilde{w}}=\oplus_{\tilde{\mu}} \dot{\mathcal{M}}_{\tilde{\mu}}^{\tilde{w}}$ be the object of $\dot{H}^{2} \operatorname{cecke}\left(\operatorname{Gr}_{G}, \check{G}\right)^{I^{0}}$, corresponding to the object $\dot{\mathbf{M}}^{\lambda} \in \dot{\operatorname{Hecke}}\left(\mathbf{U}_{\ell}, \check{G}\right)$. By Corollary 3.1.4, as an object of $\overline{\operatorname{Perv}}\left(\operatorname{Gr}_{G}\right)^{I^{0}}$,

$$
\dot{\mathcal{M}}_{\check{\mu}}^{w \cdot \check{\lambda}}:=\underset{\check{\lambda}^{\prime} \in \check{\Lambda}^{+}}{\lim } \mathcal{W}^{*, w \cdot\left(\check{\lambda}+\check{\lambda}^{\prime}-\check{\mu}\right)} \star \mathrm{IC}_{-w_{0}\left(\check{\lambda}^{\prime}\right), \operatorname{Gr}_{G}} .
$$

The maps in this inductive system come from the canonical maps

$$
\begin{aligned}
& \mathcal{W}^{*, w \cdot \check{\mu}^{\prime}} \star \mathrm{IC}_{\check{\lambda}^{\prime}, \mathrm{Gr}_{G}} \simeq j_{*, w \cdot \check{\mu}^{\prime}} \star \mathrm{IC}_{\check{\lambda}^{\prime}, \mathrm{Gr}_{G}} \rightarrow j_{*, w \cdot \check{\mu}^{\prime}} \star \mathcal{W}^{* \check{\lambda}^{\prime}} \simeq \\
& j_{*, w \cdot \check{\mu}^{\prime}} \star j_{*, \check{\lambda}^{\prime}} \star \delta_{1, \mathrm{Gr}_{G}} \simeq j_{*, w \cdot\left(\check{\mu}^{\prime}+\check{\lambda}^{\prime}\right)} \star \delta_{1, \mathrm{Gr}_{G}} \simeq \mathcal{W}^{*, w \cdot\left(\check{\mu}^{\prime}+\check{\lambda}^{\prime}\right)} .
\end{aligned}
$$

The Hecke morphisms $\dot{\mathcal{M}}_{\check{\mu}}^{\tilde{w}} \star \mathcal{V} \rightarrow \underset{\check{\nu}}{\oplus} \underline{V}(\check{\nu}) \otimes \dot{\mathcal{M}}_{\check{\mu}-\check{\nu}}^{\tilde{w}}$ for $V \in \check{G}$-mod, are given by translating the morphisms of Proposition 3.1.6 into the geometric context. Namely, let $\check{\lambda}$ be a weight large, compared to $V$. Then the sought-for morphism is

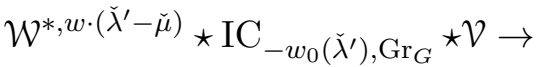

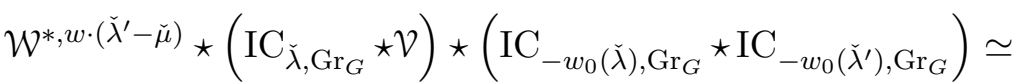

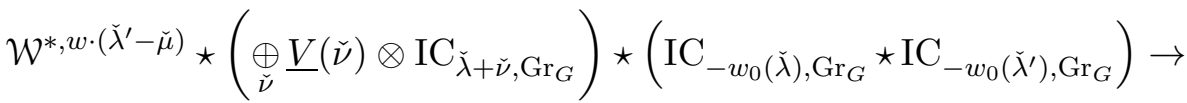

$$
\begin{aligned}
& \underset{\check{\nu}}{\oplus} \underline{V}(\check{\nu}) \otimes \mathcal{W}^{*, w\left(\check{\lambda}+\check{\lambda}^{\prime}-\check{\mu}+\check{\nu}\right)} \star\left(\mathrm{IC}_{-w_{0}(\lambda), \operatorname{Gr}_{G}} \star \mathrm{IC}_{-w_{0}\left(\check{\lambda}^{\prime}\right), \operatorname{Gr}_{G}}\right) \rightarrow \\
& \oplus_{\check{\nu}} \underline{V}(\check{\nu}) \otimes \mathcal{W}^{*, w\left(\check{\lambda}+\check{\lambda}^{\prime}-\check{\mu}+\check{\nu}\right)} \star \mathrm{IC}_{-w_{0}\left(\check{\lambda}+\check{\lambda}^{\prime}\right), \operatorname{Gr}_{G}} .
\end{aligned}
$$


MODULES OVER THE SMALL QUANTUM GROUP AND SEMI-INFINITE FLAG MANIFOLD 33

Evidently, if $\tilde{w}=w \cdot \lambda$ is such that for some $w^{\prime} \in W, l\left(w^{\prime}\right)+l(w)=l\left(w^{\prime} \cdot w\right)$, we have

$$
j_{!, w^{\prime}} \star \dot{\mathcal{M}}^{\tilde{w}} \simeq \dot{\mathcal{M}}^{w^{\prime} \cdot \tilde{w}}
$$

that it, the objects $\dot{\mathcal{M}}^{\tilde{w}}$ for different $\tilde{w}$ are obtained from one-another by convolution.

Note also that for $\check{\lambda}, \check{\mu} \in \check{\Lambda}$ with $\check{\mu}$ dominant and $\check{\lambda}$ dominant and regular,

$$
l\left(w \cdot \check{\mu} \cdot w^{-1}\right)+l(w \cdot \lambda)=l(w \cdot(\check{\mu}+\check{\lambda})) .
$$

Hence, we obtain:

Corollary 3.2.2. For $\check{\mu} \in \check{\Lambda}^{+}$there are canonical isomorphisms

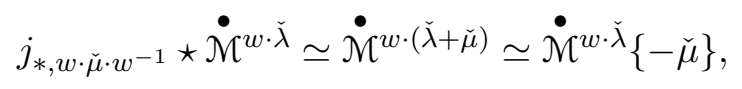

respecting the Hecke isomorphisms.

Assume now that $w \in W, \check{\lambda}, \check{\mu} \in \check{\Lambda}$ are such that $w \cdot(\check{\lambda}+\check{\mu})$ is right $W$-minimal, i.e.

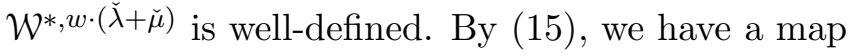

$$
\mathcal{W}^{*, w \cdot(\check{\lambda}+\check{\mu})} \rightarrow \dot{\mathcal{M}}_{-\check{\mu}}^{w \cdot \check{\lambda}}
$$

such that for $\check{\mu}^{\prime} \in \check{\Lambda}^{+}$, the diagram

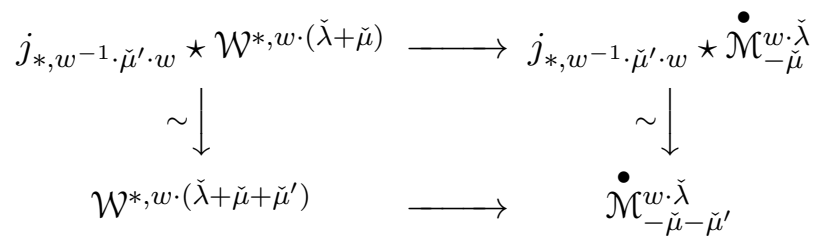

commutes.

Convolving (17) on the right with $\mathrm{IC}_{\mu^{\prime}, \mathrm{Gr}_{G}}$ we obtain the map

$$
\mathcal{W}^{*, w \cdot(\check{\lambda}+\check{\mu})} \star \mathrm{IC}_{\check{\mu}^{\prime}, \mathrm{Gr}_{G}} \rightarrow \dot{\mathcal{M}}_{-\check{\mu}}^{w \cdot \check{\lambda}} \star \mathrm{IC}_{\check{\mu}^{\prime}, \mathrm{Gr}_{G}} \simeq \underset{\check{\nu}}{\oplus} \underline{V}^{\check{\mu}^{\prime}}(\check{\nu}) \otimes \dot{\mathcal{M}}_{-\check{\nu}-\check{\mu}}^{w \cdot \check{\mu}}
$$

The above description of the Hecke morphisms implies also the following:

\section{Corollary 3.2.3.}

(1) The diagram

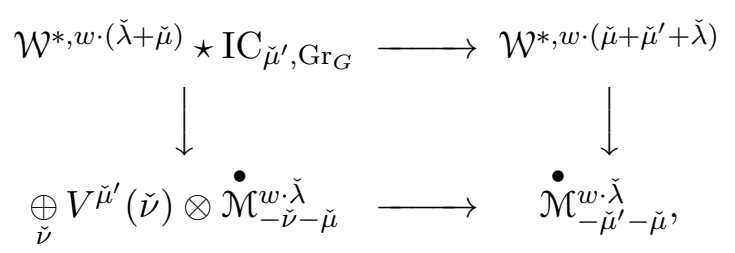

commutes, where the bottom horizontal arrow is the projection on the direct summand, corresponding to $\check{\nu}=\check{\mu}^{\prime}$.

(2) The object $\dot{\mathcal{M}}^{w \cdot \check{\lambda}} \in \dot{\mathrm{Hecke}}\left(\mathrm{Gr}_{G}, \check{G}\right)^{I^{0}}$ is universal with respect to the properties that (a) it satisfies Corollary 3.2.2, (b) it receives a map as in (17) for some $\check{\mu}$, such that (a) and (b) render the above diagram is commutative. 
If we put $w=1$ and $\check{\mu}=-\check{\lambda}$, the map in (17) identifies with

$$
\delta_{1, \mathrm{Gr}_{G}} \rightarrow \dot{\mathcal{M}}_{-\check{\lambda}}^{\check{\lambda}}
$$

Thus, we obtain a characterization of $\dot{\mathcal{M}}^{\check{\lambda}}$ in terms of $\delta_{1, \mathrm{Gr}_{G}}$.

Finally, we note that the normalizer of the torus $\check{T} \subset \check{G}$ acts on $\dot{H e c k e}\left(\operatorname{Gr}_{G}, \check{G}\right)^{I^{0}}$ by self-equivalences, modifying the Hecke morphisms. The functors, corresponding to elements of $\check{T}$ are (non-canonically) isomorphic to identity. For $w \in W$ we will denote by ${ }^{w} \dot{\mathcal{M}} \tilde{w}$ the object of $\dot{H}^{\tilde{H}}{ }^{2}\left(\mathrm{Gr}_{G}, \check{G}\right)^{I^{0}}$ obtain in this way from $\dot{\mathcal{M}}^{\tilde{w}}$; it corresponds to the object ${ }^{w} \dot{M}^{w(\lambda)} \in \dot{\mathfrak{u}}_{\ell}$-mod.

3.2.4. We will now list several facts about the objects $\dot{\mathcal{M}}^{\tilde{w}}$, most of which are formal consequences of the corresponding properties of $\dot{M}^{\lambda}$, but we will give geometric proofs for completeness.

Let $\tilde{w}=w \cdot \check{\mu}$ be an element of $W_{a f f}$, and let $\check{\lambda}$ be such that $\mathcal{L}^{w} \simeq \mathrm{IC}_{w \cdot \check{\lambda}, \mathrm{Gr}_{G}}$, in particular, $w \cdot \check{\lambda}$ is restricted. Then we have:

Proposition 3.2.5. The socle of $\dot{\mathcal{M}}^{\tilde{w}}$ is isomorphic to $\mathcal{L}^{w} \star \dot{\mathcal{R}}_{\breve{G}}\{\check{\lambda}-\check{\mu}\}$.

Proof. By Sect. 1.3.8, every irreducible in $\operatorname{Hecke}\left(\operatorname{Gr}_{G}, \check{G}\right)^{I^{0}}$ is of the form $\mathcal{L}^{w^{\prime}} \star \dot{\mathcal{R}}_{\breve{G}}\left\{\check{\mu}^{\prime}\right\}$ for some $w^{\prime} \in W$ and $\check{\mu}^{\prime} \in \check{\Lambda}$. Suppose that such an irreducible maps to $\dot{\mathcal{M}}^{w \cdot \breve{\mu}}$. By adjunction, this means that we have a map

$$
\mathcal{L}^{w^{\prime}} \rightarrow \mathcal{W}^{*, w \cdot\left(\check{\lambda}^{\prime}+\check{\mu}+\check{\mu}^{\prime}\right)} \star \mathrm{IC}_{-w_{0}\left(\check{\lambda}^{\prime}\right), \mathrm{Gr}_{G}}
$$

in $\operatorname{Perv}\left(\mathrm{Gr}_{G}\right)^{I^{0}}$ for some $\check{\lambda}^{\prime} \in \check{\Lambda}^{+}$.

The latter can be rewritten as an element in

$$
\operatorname{Hom}\left(\mathcal{L}^{w^{\prime}} \star \mathrm{IC}_{\check{\lambda}^{\prime}, \mathrm{Gr}_{G}}, \mathcal{W}^{*, w \cdot\left(\check{\lambda}^{\prime}+\check{\mu}+\check{\mu}^{\prime}\right)}\right) .
$$

By Theorem 1.3.5, and taking into account that the socle of $\mathcal{W}^{*, w} \cdot\left(\check{\lambda}^{\prime}+\check{\mu}+\check{\mu}^{\prime}\right)$ is isomorphic to $\mathrm{IC}_{w \cdot\left(\check{\lambda}^{\prime}+\check{\mu}+\check{\mu}^{\prime}\right), \mathrm{Gr}_{G}}$, this implies $w^{\prime}=w$ and $\check{\mu}^{\prime}=\check{\lambda}-\check{\mu}$.

We also obtain that the above Hom is 1-dimensional. I.e., $\mathcal{L}^{w} \star \dot{\mathcal{R}}_{\breve{G}}\{\check{\lambda}-\check{\mu}\}$ is the only irreducible that can map to $\dot{\mathcal{M}}^{\tilde{w}}$, and it appears in the socle with multiplicity 1.

\section{Proposition 3.2.6.}

(1) The object $\dot{\mathcal{M}}^{1} \in \dot{\mathrm{H} e c k e}\left(\mathrm{Gr}_{G}, \check{G}\right)^{I}$ is finitely generated. ${ }^{6}$ Its cosocle is isomorphic to $\mathcal{L}^{w_{0}} \star \dot{\mathcal{R}}_{\check{G}}\left\{\check{\rho}^{\prime}\right\}$. Moreover, all the constituents in $\operatorname{ker}\left(\dot{\mathcal{M}}^{1} \rightarrow \mathcal{L}^{w_{0}} \star \dot{\mathcal{R}}_{\breve{G}}\left\{\check{\rho}^{\prime}\right\}\right)$ are partially integrable.

(2) There exists a surjection $\dot{\mathcal{M}}^{w_{0}} \rightarrow \dot{\mathcal{R}}_{\check{G}}\{2 \check{\rho}\}$.

\footnotetext{
${ }^{6}$ Here and in the sequel, the superscript " $1 "$ in $\dot{\mathcal{M}}^{1}$ stands for the unit element in $W_{\text {aff }}$.
} 
Using (16), from point (1) of the proposition we obtain:

Corollary 3.2.7. Every $\dot{\mathcal{M}}^{\tilde{w}}$ is finitely generated (as an object of $\dot{\operatorname{Hecke}}\left(\mathrm{Gr}_{G}, \check{G}\right)^{I}$ ).

Before giving the proof of the proposition, we need to introduce the following construction. Consider a $\check{\Lambda}$-graded object of $\overline{\operatorname{Perv}}\left(\mathrm{Gr}_{G}\right)^{I^{0}}$ given by

$$
\dot{\mathcal{R}}_{\check{G}^{\prime}}:=\bigoplus_{\check{\mu}} \underset{\check{\lambda} \in \check{\Lambda}^{+}}{\lim } \mathrm{IC}_{\check{\lambda}-\check{\mu}, \operatorname{Gr}_{G}} \star \mathrm{IC}_{-w_{0}(\check{\lambda}), \operatorname{Gr}_{G}},
$$

where the maps in the inductive system are given as follows. If $\check{\lambda}^{\prime}=\check{\lambda}+\check{\nu}, \check{\nu} \in \check{\Lambda}^{+}$,

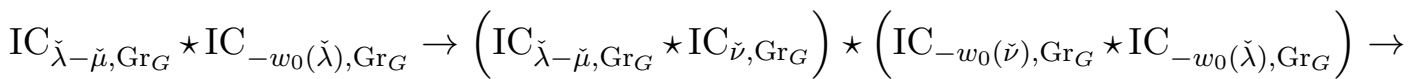

$$
\begin{aligned}
& \mathrm{IC}_{\check{\lambda}-\check{\mu}+\check{\nu}, \mathrm{Gr}_{G}} \star \mathrm{IC}_{-w_{0}(\check{\nu}+\check{\lambda}), \mathrm{Gr}_{G}} \text {. }
\end{aligned}
$$

Proposition-Construction 3.2.8. The object $\dot{\mathcal{R}}_{\breve{G}}^{\prime}$ is a Hecke eigen-sheaf, and as such, it is canonically isomorphic to $\dot{\mathcal{R}}_{\check{G}}$.

Proof. Since all the appearing perverse sheaves are spherical, we can work in the tensor category of $\operatorname{Rep}(\breve{G})$ instead of $\operatorname{Perv}\left(\operatorname{Gr}_{G}\right)^{I^{0}}$. The Hecke morphisms are given as follows. Let $\check{\lambda} \in \check{\Lambda}^{+}$be large compared to $V$. Then the sought-for map is the composition:

$$
\begin{aligned}
& V \otimes V^{\check{\lambda}^{\prime}-\check{\mu}} \otimes\left(V^{\check{\lambda}^{\prime}}\right)^{*} \rightarrow V \otimes V^{\check{\lambda}} \otimes V^{\check{\lambda}^{\prime}-\check{\mu}} \otimes\left(V^{\check{\lambda}^{\prime}}\right)^{*} \otimes\left(V^{\check{\lambda}}\right)^{*} \rightarrow \\
& \underset{\check{\nu}}{\oplus}\left(V^{\check{\lambda}^{\prime}-\check{\mu}} \otimes V^{\check{\lambda}+\check{\nu}}\right) \otimes\left(\left(V^{\check{\lambda}^{\prime}}\right)^{*} \otimes\left(V^{\check{\lambda}}\right)^{*}\right) \otimes \underline{V}(\nu) \rightarrow \underset{\check{\nu}}{\oplus} V^{\check{\lambda}+\lambda^{\prime}+\check{\nu}-\check{\mu}} \otimes\left(V^{\check{\lambda}+\check{\nu}}\right)^{*} \otimes \underline{V}(\nu) .
\end{aligned}
$$

To see that $\dot{\mathcal{R}}_{\check{G}^{\prime}}$ is isomorphic to $\dot{\mathcal{R}}_{\breve{G}}$, it is enough to notice that

$$
\operatorname{Hom}\left(V, \underset{\check{\lambda} \in \check{\lim }^{+}}{\longrightarrow} V^{\check{\lambda}-\check{\mu}} \otimes\left(V^{\check{\lambda}}\right)^{*}\right) \simeq \underset{\longrightarrow}{\lim } \operatorname{Hom}\left(V \otimes V^{\check{\lambda}}, V^{\check{\lambda}-\check{\mu}}\right) .
$$

When $\check{\lambda}$ is large with respect to $V$, the latter inductive system stabilizes to $(\underline{V})^{*}(\check{\mu})$.

Now we are ready to prove Proposition 3.2.6.

Proof. First, we claim that $\dot{\mathcal{M}}^{1}$ cannot map to any partially integrable object of the category $\dot{H}$ ecke $\left(\operatorname{Gr}_{G}, \breve{G}\right)^{I^{0}}$. Indeed, if $\dot{\mathcal{S}}$ were partially integrable and we had a non-zero map $\dot{\mathcal{M}}^{1} \rightarrow \dot{\mathcal{S}}$, we would have a non-zero map in $\operatorname{Perv}\left(\mathrm{Gr}_{G}\right)^{I}$ :

$$
\mathcal{W}^{*, \lambda} \star \mathrm{IC}_{\check{\mu}, \mathrm{Gr}_{G}} \rightarrow \mathcal{S}^{\prime}
$$

for some $\check{\lambda}, \check{\mu} \in \check{\Lambda}^{+}$and $\mathcal{S}^{\prime} \in{ }^{P I} \operatorname{Perv}\left(\mathrm{Gr}_{G}\right)^{I}$. By adjunction we would then have a map

$$
\mathcal{W}^{*, \lambda} \rightarrow \mathcal{S}^{\prime} \star \mathrm{IC}_{-w_{0}(\check{\mu}), \operatorname{Gr}_{G}}=: \mathcal{S}^{\prime \prime},
$$

with $\mathcal{S}^{\prime \prime}$ being also partially integrable. But the latter is impossible by Proposition 2.3.2(1). 
Let us now construct a map $\dot{\mathcal{M}}^{1} \rightarrow \mathcal{L}^{w_{0}} \star \dot{\mathcal{R}}_{\check{G}}\left\{\check{\rho}^{\prime}\right\}$. According to Proposition 2.3.2(1) and Theorem 1.3.5, for every dominant and regular $\check{\lambda}$ we have a canonical map

$$
\mathcal{W}^{*, \check{\lambda}} \rightarrow \mathrm{IC}_{w_{0} \cdot \check{\lambda}} \simeq \mathrm{IC}_{w_{0} \cdot \check{\rho}^{\prime}} \star \mathrm{IC}_{\check{\lambda}-\check{\rho}^{\prime}, \mathrm{Gr}_{G}} .
$$

In addition, for $\check{\mu} \in \check{\Lambda}^{+}$the diagram

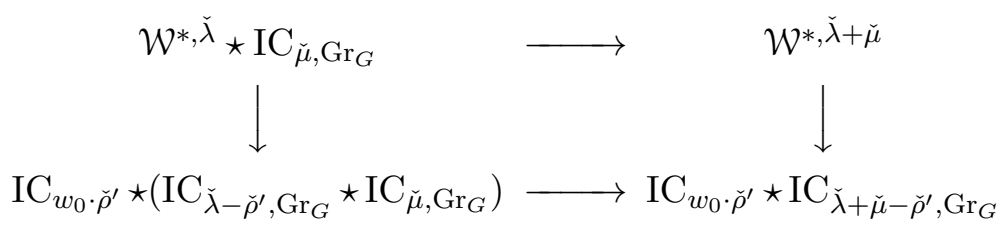

is easily seen to commute.

This defines the map of between the inductive systems:

$$
\dot{\mathcal{M}}_{\check{\mu}}^{1} \simeq \underset{\check{\lambda}}{\lim } \mathcal{W}^{*, \check{\lambda}-\check{\mu}} \star \mathrm{IC}_{-w_{0}(\check{\lambda}), \operatorname{Gr}_{G}} \rightarrow \underset{\check{\lambda}}{\lim } \mathcal{L}^{w_{0}} \star\left(\mathrm{IC}_{\check{\lambda}-\check{\mu}-\check{\rho}^{\prime}} \star \mathrm{IC}_{-w_{0}(\check{\lambda}), \operatorname{Gr}_{G}}\right),
$$

and the latter identifies with $\mathcal{L}^{w_{0}} \star \dot{\mathcal{R}}_{\check{G}}\left\{\check{\rho}^{\prime}\right\}$, by Proposition-Construction 3.2.8.

It is straightforward to check that the above map respects the Hecke morphisms, i.e., we obtained the desired map in $\dot{H}$ ecke $\left(\mathrm{Gr}_{G}, \check{G}\right)^{I^{0}}$. Moreover, from Proposition 2.3.2(2) it follows that the kernel of the map $\dot{\mathcal{M}}^{1} \rightarrow \mathcal{L}^{w_{0}} \star \dot{\mathcal{R}}_{\breve{G}}\left\{\check{\rho}^{\prime}\right\}$ is partially integrable.

To finish the proof of the first part of the proposition, it remains to show that the map

$$
\mathcal{W}^{*, \lambda} \star \dot{\mathcal{R}}_{\check{G}}\{\check{\lambda}\} \rightarrow \dot{\mathcal{M}}^{1}
$$

is surjective for some (and, in fact, every) regular $\check{\lambda}$. By construction, the composition

$$
\mathcal{W}^{*, \lambda} \star \dot{\mathcal{R}}_{\breve{G}}\{\check{\lambda}\} \rightarrow \dot{\mathcal{M}}^{1} \rightarrow \mathcal{L}^{w_{0}} \star \dot{\mathcal{R}}_{\check{G}}\left\{\check{\rho}^{\prime}\right\}
$$

is surjective. Hence, by the above, the cokernel of (19) is partially integrable, and hence, is zero.

To prove the second assertion of the proposition, recall from Proposition 2.3.4 that for $\check{\lambda}$ large we have a map

$$
\mathcal{W}^{*, w_{0} \cdot \check{\lambda}} \rightarrow \mathrm{IC}_{\check{\lambda}-2 \check{\rho}},
$$

defined up to a scalar. Moreover, from the construction of this map one deduces that the square

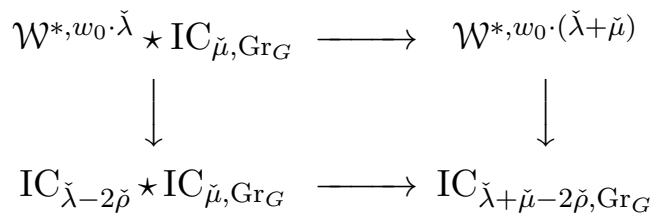

commutes (up to a scalar). We can normalize the maps in (20) to make such diagrams commutative. 
MODULES OVER THE SMALL QUANTUM GROUP AND SEMI-INFINITE FLAG MANIFOLD 37

This gives us a map of inductive systems

$$
\dot{\mathcal{M}}_{\check{\mu}}^{w_{0}} \simeq \underset{\check{\lambda}}{\lim } \mathcal{W}^{*, w_{0} \cdot(\check{\lambda}-\check{\mu})} \star \mathrm{IC}_{-w_{0}(\check{\lambda}), \mathrm{Gr}_{G}} \rightarrow \underset{\check{\lambda}}{\lim } \mathrm{IC}_{\check{\lambda}-\check{\mu}-2 \check{\rho}} \star \mathrm{IC}_{-w_{0}(\check{\lambda}), \operatorname{Gr}_{G}},
$$

and the latter identifies with $\dot{\mathcal{R}}_{\breve{G}}\{2 \check{\rho}\}$.

3.2.9. A dual description. Recall that over the small quantum group, the baby Verma modules can be expressed through the baby co-Verma modules and a twist by elements of the Weyl group. We would like to establish this fact geometrically as well. By (16), it suffices to consider the case of just $\dot{\mathcal{M}}^{1}$.

Proposition 3.2.10. We have an isomorphism

$$
\mathbb{D}\left(\dot{\mathcal{M}}^{1}\right) \simeq\left({ }^{w_{0}} \dot{\mathcal{M}}^{w_{0}}\right)\{2 \check{\rho}\} .
$$

Since the convolution functors commute with Verdier duality, from (16),we obtain:

\section{Corollary 3.2.11.}

$$
\mathbb{D}\left(\dot{\mathcal{M}}^{w \cdot \check{\mu}}\right) \simeq{ }^{w_{0}} \dot{\mathcal{M}}^{w \cdot w_{0} \cdot\left(w_{0}(\check{\mu})+2 \check{\rho}\right)}
$$

Combining this with Proposition 3.2.5, we also obtain:

Corollary 3.2.12. The cosocle of every $\dot{\mathcal{M}}^{w \cdot \check{\mu}}$ is simple and isomorphic to

$$
\mathrm{IC}_{w \cdot w_{0} \cdot \check{\lambda}, \operatorname{Gr}_{G}} \star \dot{\mathcal{R}}_{\check{G}}\left\{w_{0}(\check{\lambda})-\check{\mu}+2 \check{\rho}\right\},
$$

where $\check{\lambda} \in \check{\Lambda}^{+}$is such that $w \cdot w_{0} \cdot \check{\lambda}$ is restricted.

Proof. By Proposition 3.2.6(1) and Proposition 3.2.5, it is enough to construct a map

$$
\dot{\mathcal{M}}^{1} \rightarrow \mathbb{D}\left(\left({ }^{w_{0}} \dot{\mathcal{M}}^{w_{0}}\right)\{2 \check{\rho}\}\right)
$$

such that the composition

$$
\dot{\mathcal{R}}_{\check{G}} \rightarrow \dot{\mathcal{M}}^{1} \rightarrow \mathbb{D}\left(\left({ }^{w_{0}} \dot{\mathcal{M}}^{w_{0}}\right)\{2 \check{\rho}\}\right)
$$

equals (up to a scalar) the map, obtained by duality from Proposition 3.2.6(2), and such that the composition

$$
\dot{\mathcal{M}}^{1} \rightarrow \mathbb{D}\left(\left({ }^{w_{0}} \dot{\mathcal{M}}^{w_{0}}\right)\{2 \check{\rho}\}\right) \rightarrow \mathcal{L}^{w_{0}}\left\{\check{\rho}^{\prime}\right\} \star \dot{\mathcal{R}}_{\check{G}}
$$

equals the map of Proposition 3.2.6(1).

By Corollary 3.2.2 and duality

$$
j_{!, w_{0}(\check{\lambda})} \star \mathbb{D}\left(\left({ }^{w_{0}} \dot{\mathcal{M}}^{w_{0}}\right)\{2 \check{\rho}\}\right) \simeq \mathbb{D}\left(\left({ }^{w_{0}} \dot{\mathcal{M}}^{w_{0}}\right)\{2 \check{\rho}\}\right)\left\{w_{0}(\check{\lambda})\right\}
$$


Hence, by Corollary 3.2.3, to construct a map as in (21), we must construct a map $\dot{\mathcal{R}}_{\breve{G}} \rightarrow \mathbb{D}\left(\left({ }^{w_{0}} \dot{\mathcal{M}}^{w_{0}}\right)\{2 \check{\rho}\}\right)$ and check the commutativity of the corresponding diagram.

By duality, the above amounts to a map $\left({ }^{w_{0}} \dot{\mathcal{M}}^{w_{0}}\right)\{2 \check{\rho}\} \rightarrow \dot{\mathcal{R}}_{\breve{G}}$. By the definition of the twisting functors, the existence of the latter map follows from Proposition 3.2.6(2). We need to check the commutativity of the following diagram:

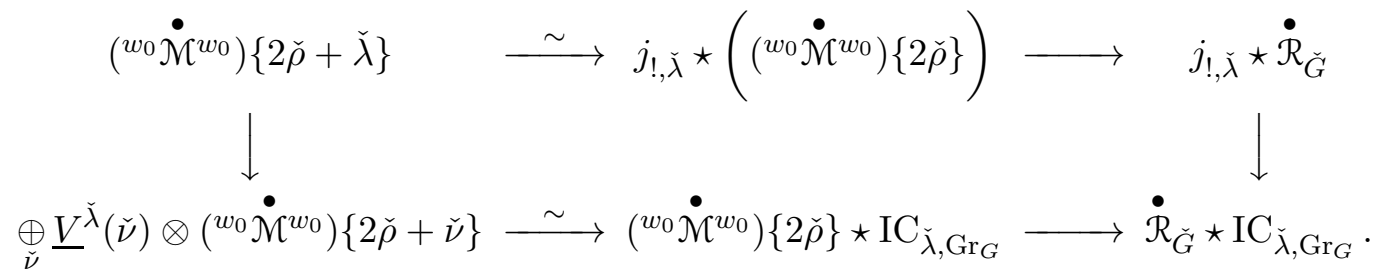

Recalling the definition of the arrows, we arrive to the following diagram, defined for $\check{\mu}$ large:

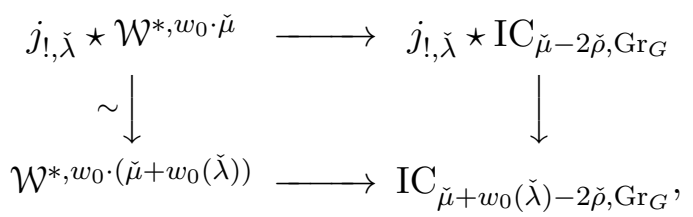

where the right vertical arrow is the composition

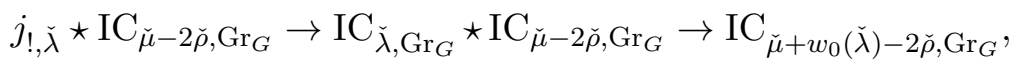

where the second arrow is obtained by adjunction from

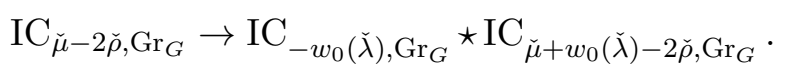

The commutativity of the latter diagram follows from the construction of the arrow in Proposition 2.3.4.

By construction, the condition on the composed map from (22) is satisfied. It remains to verify the condition in (23). The latter amounts to showing that the arrow

$$
\mathrm{IC}_{w_{0} \cdot\left(\check{\mu}+w_{0}(\check{\lambda})\right), \operatorname{Gr}_{G}} \rightarrow \mathcal{W}^{*, w_{0} \cdot\left(\check{\mu}+w_{0}(\check{\lambda})\right)} \simeq j_{!, \check{\lambda}} \star \mathcal{W}^{*, w_{0} \cdot \check{\mu}} \rightarrow j_{!, \check{\lambda}} \star \mathrm{IC}_{\check{\mu}-2 \check{\rho}, \operatorname{Gr}_{G}}
$$

equals (up to a scalar) the map

$$
\begin{aligned}
& \mathrm{IC}_{w_{0} \cdot\left(\check{\mu}+w_{0}(\check{\lambda})\right), \mathrm{Gr}_{G}} \simeq \mathrm{IC}_{w_{0} \cdot \check{\rho}^{\prime}, \mathrm{Gr}_{G}} \star \mathrm{IC}_{\check{\mu}+w_{0}(\check{\lambda})-\check{\rho}^{\prime}, \mathrm{Gr}_{G}} \rightarrow
\end{aligned}
$$

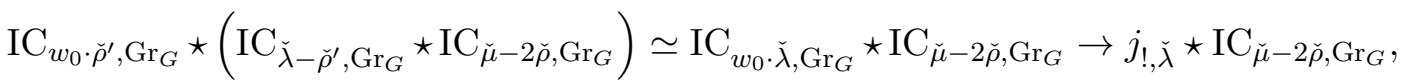

where the last arrow comes by duality from Proposition 2.3.2, and the second arrow is obtained by adjunction from

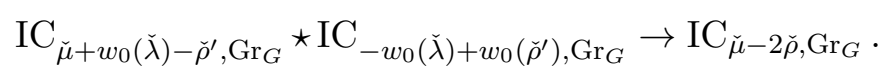

By construction, both these maps are non-zero. Now our assertion follows from the fact, that $\mathrm{IC}_{w_{0} \cdot \check{\lambda}, \mathrm{Gr}_{G}}$ is the only non-partially integrable constituent of $\mathcal{W}^{!}, \check{\lambda}$, which implies that $\mathrm{IC}_{w_{0} \cdot\left(\check{\mu}+w_{0}(\check{\lambda})\right), \operatorname{Gr}_{G}}$ appears with multiplicity one in the Jordan-Hölder series

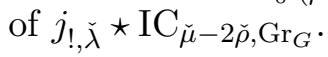


MODULES OVER THE SMALL QUANTUM GROUP AND SEMI-INFINITE FLAG MANIFOLD 39

3.2.13. Non-graded version and presentation as a quotient. Our present goal is to prove geometrically that $\dot{\mathcal{M}}^{\tilde{w}}$ can be presented as a quotient, as in Sect. 3.1.7. For that it will be convenient to consider the corresponding non-graded version, $\mathcal{M}{ }^{\tilde{w}} \in \operatorname{Hecke}\left(\operatorname{Gr}_{G}, \breve{G}\right)^{I}$. If $\tilde{w}=w \cdot \check{\lambda}$ and $\tilde{w}^{\prime}=w \cdot \check{\lambda}^{\prime}$, then, evidently, $\mathcal{M}^{\tilde{w}} \simeq \mathcal{M}^{\tilde{w}^{\prime}}$.

For $\mathbf{g} \in \check{G}$ we will denote by ${ }^{\mathbf{g}} \mathcal{M}^{\tilde{w}}$ the corresponding twist of $\mathcal{M}^{\tilde{w}}$; for $\mathbf{g}=w \in W$ we recover the objects ${ }^{w} \mathcal{M}{ }^{\tilde{w}}$. We will denote by ${ }^{\tilde{}} \mathcal{M} \mathfrak{N}^{\tilde{w}}$ the universal family of ${ }^{\mathbf{g}} \mathcal{M}^{\tilde{w}}$ over $\mathcal{O}_{\check{G}}$.

Lemma 3.2.14.

(1) As an object of $\overline{\operatorname{Perv}}\left(\mathrm{Gr}_{G}\right)^{I}, \mathcal{M}^{\tilde{w}}$ admits a unique action of the algebraic group $\check{B}^{-}$, such that

- If $\tilde{w}=w \cdot \check{\lambda}$, in terms of (15), the image of $\mathcal{W}^{*, \check{\lambda}+\check{\lambda}^{\prime}}$ in $\mathcal{M}^{\tilde{w}}$ transforms according to the $\check{B}^{-}$-character $-\check{\lambda}^{\prime}$.

- The Hecke isomorphisms

$$
\mathcal{M}^{\tilde{w}} \star \mathcal{V} \simeq \underline{V} \otimes \mathcal{M}^{\tilde{w}}
$$

intertwine the action of $\check{B}^{-}$on the left-hand side, obtained by transport of structure and the diagonal action of $\check{B}^{-}$on the right-hand side.

$$
\operatorname{Hom}_{\check{B}^{-}}\left(\mathbf{C}^{-\check{\mu}} \otimes \operatorname{Res}_{\check{B}^{-}}^{\check{G}}\left(V^{\check{\lambda}^{\prime}}\right), \mathcal{M} \mathcal{T}^{\tilde{w}}\right) \simeq \mathcal{W}^{w \cdot(\check{\lambda}+\check{\mu})} \star \mathrm{IC}_{-w_{0}\left(\check{\lambda}^{\prime}\right), \operatorname{Gr}_{G}},
$$

if $w \cdot(\check{\lambda}+\check{\mu}) \in W_{\text {aff }}$ is right $W$-minimal, and 0 otherwise.

Note that the action of $\check{T} \subset \check{B}^{-}$on $\mathcal{M} \tilde{w}^{\tilde{w}}$ as an object of $\overline{\operatorname{Perv}}\left(\operatorname{Gr}_{G}\right)^{I}$ comes from the grading on $\dot{\mathcal{M}}^{\tilde{w}}$.

The first assertion of the lemma means that, as an object of $\operatorname{Hecke}\left(\operatorname{Gr}_{G}, \check{G}\right)^{I}, \mathcal{M}^{\tilde{w}}$ is $\check{B}^{-}$-equivariant, i.e., that the $\mathcal{O}_{\check{G}^{-}}$-family $\check{G}_{\mathcal{M}}{ }^{\tilde{w}}$ acquires a $\check{B}^{-}$-action, covering that on $\mathcal{O}_{\check{G}}$. Alternatively, a structure of a $\check{B}^{-}$-equivariant object on some $\mathcal{N} \in \operatorname{Hecke}\left(\operatorname{Gr}_{G}, \check{G}\right)^{I}$ is a $\check{B}^{-}$-action on $\mathcal{N}$ as an object of $\overline{\operatorname{Perv}}\left(\mathrm{Gr}_{G}\right)^{I}$, which is compatible with the Hecke morphisms in the natural sense.

Let us denote this category by Hecke $\left(\mathrm{Gr}_{G}, \check{G}_{\check{B}^{-}}^{I}\right.$. Let us consider also the category Hecke $\left(\operatorname{Gr}_{G}, \check{G}\right)_{\check{G}}^{I}$ of $\check{G}$-equivariant objects of Hecke $\left(\mathrm{Gr}_{G}, \check{G}\right)^{I}$; it is canonically equivalent to $\overline{\operatorname{Perv}}\left(\operatorname{Gr}_{G}\right)^{I}$.

Let us now recall the following general construction. Let $\mathcal{N}$ be a an object of Hecke $\left(\operatorname{Gr}_{G}, \check{G}\right)_{\check{B}^{-}}^{I}$. We claim that it gives rise to a functor

$$
\mathrm{QCoh}\left(\check{G} / \check{B}^{-}\right) \rightarrow \operatorname{Hecke}\left(\mathrm{Gr}_{G}, \check{G}\right)^{I} .
$$

Indeed, given $\mathcal{K} \in \mathrm{Q} \operatorname{Coh}\left(\check{G} / \check{B}^{-}\right)$, which we will view as a $\check{B}^{-}$-equivariant $\mathcal{O}_{\check{G}^{-}}$-module, consider the tensor product $\mathcal{K} \underset{\mathcal{O}_{\check{G}}}{\otimes} \check{G}_{\mathcal{N}}$. This is an object of Hecke $\left(\mathrm{Gr}_{G}, \check{G}\right)^{I}$, endowed with an action of $\check{B}^{-}$, and we set

$$
\mathcal{K} * \mathcal{N}:=\left(\mathcal{K} \underset{\mathcal{O}_{\breve{G}} \otimes}{\otimes} \mathcal{N}\right)^{\check{B}^{-}}
$$


The underlying object of $\overline{\operatorname{Perv}}\left(\mathrm{Gr}_{G}\right)^{I}$ is given by $(\mathcal{K} \otimes \mathcal{N})^{\check{B}^{-}}$.

Suppose now that $\mathcal{K}$ is an object of $\mathrm{QCoh}\left(\check{G} / \check{B}^{-}\right)_{\check{G}}$. Then, by construction $\mathcal{K} * \mathcal{N}$ belongs to Hecke $\left(\mathrm{Gr}_{G}, \check{G}\right)_{\breve{G}}^{I} \simeq \overline{\operatorname{Perv}}\left(\operatorname{Gr}_{G}\right)^{I}$.

Lemma 3.2.15. The functor $\mathcal{N} \mapsto \mathcal{O}_{\check{G} / \check{B}^{-}} * \mathcal{N}:$ Hecke $\left(\operatorname{Gr}_{G}, \check{G}_{\check{B}^{-}}^{I} \rightarrow \overline{\operatorname{Perv}}\left(\operatorname{Gr}_{G}\right)^{I}\right.$ is the right adjoint of the forgetful functor

$$
\overline{\operatorname{Perv}}\left(\mathrm{Gr}_{G}\right)^{I} \simeq \operatorname{Hecke}\left(\mathrm{Gr}_{G}, \check{G}\right)_{\check{G}}^{I} \rightarrow \operatorname{Hecke}\left(\operatorname{Gr}_{G}, \check{G}\right)_{\check{B}^{-}}^{I} .
$$

Note that the functor $\mathcal{N} \mapsto \mathcal{O}_{\check{G} / \check{B}^{-}} * \mathcal{N}:$ Hecke $\left(\operatorname{Gr}_{G}, \check{G}_{\check{B}^{-}}^{I} \rightarrow \overline{\operatorname{Perv}}\left(\operatorname{Gr}_{G}\right)^{I}\right.$ can be tautologically rewritten as $\mathcal{N} \mapsto \mathcal{N}^{\check{B}^{-}}$. The following is a translation of the Borel-BottWeil theorem:

Proposition 3.2.16. Assume that $\tilde{w}$ is right $W$-minimal. Then $\mathcal{O}_{\check{G} / \check{B}^{-}} * \mathcal{M} \mathcal{N}^{\tilde{w}} \simeq \mathcal{W}^{*, \tilde{w}}$, and for $i>0$

$$
\mathrm{R}^{i}\left(\check{B}^{-}, \mathcal{M}^{\tilde{w}}\right)=0 .
$$

Proof. The first assertion of the proposition is immediate from Lemma 3.2.14(2). To prove the second assertion, note that if $\mathcal{N}$ is any Artinian $\check{B}^{-}$-equivariant object of Hecke $\left(\mathrm{Gr}_{G}, \check{G}\right)^{I}$, there exists $\check{\mu} \in \check{\Lambda}^{+}$large enough, so that $\mathrm{R}^{i}\left(\check{B}^{-}, \mathbf{C}^{\check{\mu}} \otimes \mathcal{N}\right)=0$ for $i>0$. This follows from the fact that the functor of derived $\check{B}^{-}$-invariants has a finite cohomological dimension, and any Artinian object of $\operatorname{Hecke}\left(\operatorname{Gr}_{G}, \check{G}\right)_{\check{B}^{-}}^{I}$, admits a left resolution, whose terms are of the form $\mathcal{F} \star \mathcal{R}_{\breve{G}} \otimes U$, where $\mathcal{F} \in \operatorname{Perv}\left(\operatorname{Gr}_{G}\right)^{I}$, and $U$ is a finite-dimensional representation of $\check{B}^{-}$.

Hence, for a given $w \in W$ and $\check{\lambda}^{\prime} \in \check{\Lambda}^{+}$large enough,

$$
\operatorname{RInv}\left(\check{B}^{-}, \mathcal{M}^{w \cdot \check{\lambda}^{\prime}}\right) \simeq \mathcal{W}^{*, w \cdot \check{\lambda}^{\prime}} .
$$

Note that the functor $\operatorname{RInv}\left(\check{B}^{-}, \cdot\right): \mathrm{D}\left(\operatorname{Hecke}\left(\mathrm{Gr}_{G}, \check{G}_{\check{B}^{-}}\right) \rightarrow \mathrm{D}\left(\mathrm{Gr}_{G}\right)^{I}\right.$ commutes with the action of $\mathrm{D}\left(\mathrm{Fl}_{G}\right)^{I}$ by convolutions. It suffices to remark that if $\check{\lambda}^{\prime}-\check{\lambda} \in \check{\Lambda}^{+}$,

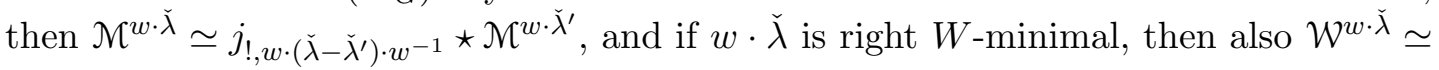

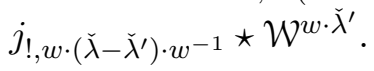

Corollary 3.2.17. Let $\tilde{w} \in W_{\text {aff }}$ be right maximal with respect to $W$. Then for $i \neq \operatorname{dim}(\mathfrak{n}), H^{i}\left(\check{B}^{-}, \mathcal{M} \tilde{W}^{\tilde{w}}\right)=0$, and

$$
H^{\operatorname{dim}(\mathfrak{n})}\left(\check{B}^{-}, \mathcal{M}^{\tilde{w}}\right) \simeq \mathcal{W}^{!, \tilde{w} \cdot w_{0}} .
$$

Proof. Let $\tilde{w}=w \cdot \check{\lambda}$, and let $\check{\mu} \in \check{\Lambda}^{+}$be such that $w \cdot(\check{\lambda}+\check{\mu})$ is left minimal with respect to $W$. Then:

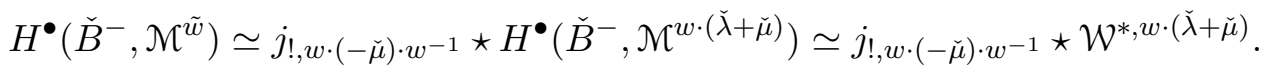

The latter is isomorphic to

$$
j_{!}, \tilde{w} \star \delta_{1, \mathrm{Gr}_{G}} \simeq \mathcal{W}^{!}, \tilde{w} \cdot w_{0}[-\operatorname{dim}(\mathfrak{n})] .
$$


Let now $\ldots \rightarrow \mathcal{P}_{1} \rightarrow \mathcal{P}_{0} \rightarrow \mathbb{C}_{\check{B}^{-}}$be a left resolution of the skyscraper on $\check{G} / \check{B}^{-}$, as in Sect. 3.1.7, where each $\mathcal{P}_{i}$ has the form $\mathcal{O}\left(-\check{\mu}_{i}\right) \otimes U^{i}$, where $U^{i}$ are vector spaces.

Let $\tilde{w}$ be $w \cdot \check{\lambda}$. Tensoring by the line bundle $\mathcal{O}\left(\check{\lambda}^{\prime}\right)$, we can ensure that $\check{\lambda}+\check{\lambda}^{\prime}-\check{\mu}_{i}$ are such that $w \cdot\left(\check{\lambda}+\check{\lambda}^{\prime}-\check{\mu}_{i}\right)$ is right $W$-minimal for $i=0, \ldots, \operatorname{dim}(\mathfrak{n})$. We obtain that the complex

$$
\left(\mathcal{P}_{1} \otimes \mathcal{O}\left(\check{\lambda}^{\prime}\right)\right) * \mathcal{M} \tilde{\mathcal{T}}^{\tilde{w}} \rightarrow\left(\mathcal{P}_{0} \otimes \mathcal{O}\left(\check{\lambda}^{\prime}\right)\right) * \mathcal{M} \tilde{\mathcal{T}}^{\tilde{w}} \rightarrow \mathbb{C}_{\check{B}^{-}} * \mathcal{M} \mathfrak{T}^{\tilde{w}} \rightarrow 0
$$

is exact. However, $\mathbb{C}_{\check{B}^{-}} * \mathcal{M}^{\tilde{w}} \simeq \mathcal{M}^{\tilde{w}}$, and

$$
\mathcal{O}(\check{\mu}) * \mathcal{M}^{w \cdot \check{\lambda}} \simeq \mathcal{W}^{*, w \cdot(\check{\mu}+\check{\lambda})},
$$

by Proposition 3.2.16, provided that $w \cdot(\check{\mu}+\check{\lambda})$ is right $W$-minimal. Thus, we arrive to the same conclusion as in Proposition 3.1.8.

3.2.18. Hereditary property. In this subsection we will prove the following:

Theorem 3.2.19. $\operatorname{Ext}_{\operatorname{Hecke}^{i}\left(\operatorname{Gr}_{G}, \check{G}\right)^{I^{0}}}\left(\mathbb{D}(\dot{\mathcal{M}} \tilde{w}), \dot{\mathcal{M}}^{\tilde{w}^{\prime}}\right)=0$ for $i>0$ and any $\tilde{w}, \tilde{w}^{\prime} \in W_{\text {aff }}$, and $\operatorname{Hom}\left(\mathbb{D}\left(\dot{\mathcal{M}}^{\tilde{w}}\right), \dot{\mathcal{M}}^{\tilde{w}^{\prime}}\right)$ is zero if $\tilde{w} \neq \tilde{w}^{\prime}$, and 1-dimensional otherwise.

This theorem follows immediately from Theorem 1.3.9 due to the corresponding property of baby co-Verma modules over the small quantum group. Here we will discuss a geometric proof of this fact, which the rest of this subsection is devoted to. In the course of the proof we will introduce another important object-the Wakimoto sheaf.

Let Hecke $\left(\mathrm{Gr}_{G}, \check{G}\right)_{\breve{B}}^{I^{0}}$ be the category of $\check{B}$-equivariant objects in $\operatorname{Hecke}\left(\mathrm{Gr}_{G}, \check{G}\right)^{I^{0}}$. By Proposition 3.2.10, $\mathbb{D}\left(\mathcal{M} w^{\tilde{w}}\right)$ is naturally an object of Hecke $\left(\operatorname{Gr}_{G}, \check{G}_{\breve{B}}\right)_{\breve{B}}^{I^{0}}$.

For $\tilde{w}=w \cdot \check{\mu}$ consider the following object Wak ${ }^{\tilde{w}}$ of Hecke $\left(\operatorname{Gr}_{G}, \check{G}\right)_{\check{B}}^{I^{0}}$. It is defined as

$$
\underset{\check{\nu} \in \check{\Lambda}}{\lim _{\longrightarrow}} \mathcal{W}^{*, w \cdot(\check{\nu}+\check{\mu})} \star \mathcal{R}_{\breve{G}}\{\check{\nu}\}
$$

where the maps in the inductive system, defined for $\check{\nu}^{\prime}-\check{\nu}=\check{\lambda} \in \check{\Lambda}^{+}$, are given by

$$
\begin{aligned}
& \mathcal{W}^{*, w \cdot(\check{\nu}+\check{\mu})} \star \mathcal{R}_{\check{G}}\{\check{\nu}\} \rightarrow \mathcal{W}^{*, w \cdot(\check{\nu}+\check{\mu})} \star \mathcal{R}_{\check{G}}\{\check{\nu}+\check{\lambda}\} \otimes \underline{V}^{\check{\lambda}}(\check{\lambda}) \rightarrow \\
& \rightarrow \mathcal{W}^{*, w \cdot(\check{\nu}+\check{\mu})} \star \mathcal{V}^{\check{\lambda}} \star \mathcal{R}_{\check{G}}\{\check{\nu}+\check{\lambda}\} \rightarrow \mathcal{W}^{*, w \cdot(\check{\nu}+\check{\mu}+\check{\lambda})} \star \mathcal{R}_{\check{G}}\{\check{\nu}+\check{\lambda}\} .
\end{aligned}
$$

Note that the forgetful functor Hecke $\left(\mathrm{Gr}_{G}, \check{G}\right)_{\check{B}}^{I^{0}} \rightarrow$ Hecke $\left(\mathrm{Gr}_{G}, \check{G}\right)^{I^{0}}$ admits a natural right adjoint given by $\mathcal{N} \mapsto \mathcal{O}_{\check{B}} \underset{\mathcal{O}_{\breve{G}}}{\otimes} \check{G} \mathcal{N}$. Similarly, the functor Hecke $\left(\operatorname{Gr}_{G}, \check{G}\right)_{\check{B}}^{I^{0}} \rightarrow$ Hecke $\left(\operatorname{Gr}_{G}, \check{G}\right)^{I^{0}}$ admits a right adjoint $\mathcal{N} \mapsto \mathcal{O}_{\check{N}} \underset{\mathcal{O}_{\check{G}}}{\otimes} \check{G} \mathcal{N}$.

Lemma 3.2.20.

$$
\mathrm{Wak}^{\tilde{w}} \simeq \mathcal{O}_{\check{N}} \underset{\mathcal{O}_{\breve{G}}}{\otimes} \check{G}\left(\dot{\mathcal{M}}^{\tilde{w}}\right)
$$


Hence, we obtain

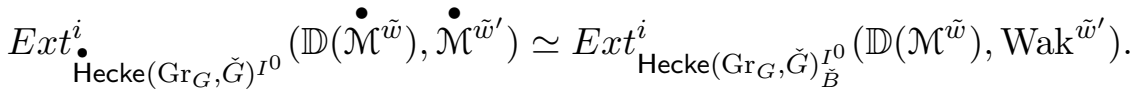

By the Artinian property and taking into account Corollary 3.2.11, to prove Theorem 3.2.19, it is sufficient to show that

$$
\left.\operatorname{Ext}_{\text {Hecke }\left(\operatorname{Gr}_{G}, \check{G}\right)_{\check{B}}^{I^{0}}}^{i}{ }^{w_{0}} \mathcal{M}^{w \cdot \check{\mu}}, \mathcal{W}^{w^{\prime} \cdot\left(\check{\lambda}+\check{\mu}^{\prime}\right)} \star \mathcal{R}_{\breve{G}}\{\check{\lambda}\}\right)=0
$$

unless $i=0, w^{\prime}=w \cdot w_{0}$ and $\check{\mu}^{\prime}=w_{0}(\check{\mu})+2 \check{\rho}$, whenever $\check{\lambda}$ is deep in $\check{\Lambda}^{+}$.

Lemma 3.2.21. For $\mathcal{N} \in \operatorname{Hecke}\left(\operatorname{Gr}_{G}, \check{G}\right)_{\check{B}}^{I^{0}}$ and $\mathcal{F} \in \operatorname{Hecke}\left(\operatorname{Gr}_{G}, \check{G}\right)^{I^{0}}$,

$$
R H o m_{\operatorname{Hecke}\left(\operatorname{Gr}_{G}, \check{G}\right)_{\check{B}}^{I^{0}}}\left(\mathcal{N}, \mathcal{F} \star \mathcal{R}_{\breve{G}}\right) \simeq R H o m_{\operatorname{Hecke}\left(\operatorname{Gr}_{G}, \check{G}\right)^{I^{0}}}(\operatorname{RCoinv}(\check{B}, \mathcal{N}), \mathcal{F}) .
$$

Proof. It is sufficient to prove the assertion in the case when $\mathcal{N} \simeq \mathcal{F}^{1} \star \dot{R} \otimes U$, where $U$ is a representation of $\check{B}$. In this case, it amounts to the following adjunction, which is a corollary of the Serre duality on $\breve{G} / \check{B}$ :

$$
\operatorname{RHom}_{\check{B}}\left(U, \operatorname{Res}_{\breve{B}}^{\check{G}}(V)\right) \simeq \operatorname{RHom}_{\breve{G}}\left(\operatorname{RCoinv}\left(\check{B}, \mathcal{O}_{\breve{G}} \otimes U\right), V\right)
$$

Lemma 3.2.22. For $\check{\lambda}$ deep in the dominant chamber,

$$
\operatorname{RCoinv}\left(\check{B},\left({ }^{w_{0}} \mathcal{M}^{w \cdot \check{\mu}}\right)\{-\check{\lambda}\}\right) \simeq \mathcal{W}^{!, w \cdot w_{0}\left(\check{\lambda}-w_{0}(\check{\mu})+2 \check{\rho}\right)}
$$

Proof. First,

$$
\operatorname{RCoinv}\left(\check{B},\left({ }^{w_{0}} \mathcal{M}^{w \cdot \check{\mu}}\right)\{-\check{\lambda}\}\right) \simeq \operatorname{RCoinv}\left(\check{B}^{-}, \mathcal{M}^{w \cdot \check{\mu}}\left\{-w_{0}(\check{\lambda})\right\}\right) .
$$

Note that for $\mathcal{N} \in \operatorname{Hecke}\left(\mathrm{Gr}_{G}, \check{G}\right)_{\check{B}^{-}}^{I^{0}}$,

$$
\operatorname{RCoinv}\left(\check{B}^{-}, \mathcal{N}\right) \simeq \operatorname{RInv}\left(\check{B}^{-}, \mathcal{N}\right)\{2 \check{\rho}\}[\operatorname{dim}(\mathfrak{n})] .
$$

Hence, the expression in the lemma is isomorphic to

$$
\operatorname{RInv}\left(\check{B}^{-}, \mathcal{M}^{w \cdot \check{\mu}}\left\{-w_{0}(\check{\lambda})+2 \check{\rho}\right\}\right)[\operatorname{dim}(\mathfrak{n})] \simeq \mathcal{W}^{!}, w \cdot w_{0} \cdot\left(w_{0}(\check{\mu})+\check{\lambda}+2 \check{\rho}\right),
$$

by Corollary 3.2.17.

Thus, we obtain that the expression in (24) is isomorphic to

$$
\operatorname{Ext}_{\text {Hecke }\left(\operatorname{Gr}_{G}, \check{G}\right)^{I^{0}}}^{i}\left(\mathcal{W}^{!}, w \cdot w_{0}\left(\check{\lambda}+w_{0}(\check{\mu})+2 \check{\rho}\right), \mathcal{W}^{*, w^{\prime} \cdot\left(\check{\mu}^{\prime}+\check{\lambda}\right)}\right),
$$

for which the vanishing assertion is manifest. 
MODULES OVER THE SMALL QUANTUM GROUP AND SEMI-INFINITE FLAG MANIFOLD 43

3.2.23. An application: 2-sided BGG resolution. We will use the geometric interpretation of baby co-Verma modules to prove the following result:

Theorem 3.2.24. There exists an exact complex $\mathcal{B}_{\frac{\infty}{2}}$ of objects of $\dot{\operatorname{Hecke}}\left(\mathrm{Gr}_{G}, \check{G}\right)^{I}$, whose $n$-th term is

$$
\bigoplus_{\tilde{w} \in W_{a f f}, l^{\frac{\infty}{2}}(\tilde{w})=n} \dot{\mathcal{M}}^{\tilde{w}} .
$$

We remind that for $\tilde{w}=w \cdot \check{\lambda} \in W_{a f f}$, its semi-infinite length $l^{\frac{\infty}{2}}(\tilde{w})$ is defined as $l(w \cdot(\check{\lambda}+\check{\mu}))-l(\check{\mu})$ for some (or all) large $\check{\mu} \in \check{\Lambda}^{+}$.

Of course, using the equivalence between $\dot{H}_{\text {Hecke }}\left(\mathrm{Gr}_{G}, \check{G}\right)^{I}$ and $\dot{\mathfrak{u}}_{\ell}-\overline{\bmod }$, we obtain the corresponding exact complex consisting of baby co-Verma modules over $\dot{\mathfrak{u}}_{\ell}$. The rest of this subsection os devoted to the proof of this theorem.

Let $\mathcal{B}_{\mathrm{Gr}_{G}}$ be the Cousin complex on $\mathrm{Gr}_{G}$. I.e., this is an exact complex of perverse sheaves on $\mathrm{Gr}_{G}$, living in positive degrees, whose $n$-th term is given by

$$
\bigoplus_{\tilde{w} \in W_{a f f} / W, l(\tilde{w})=n} \mathcal{W}^{*, \tilde{w}}
$$

For $\check{\mu} \in \check{\Lambda}^{+}$consider the complex $\mathcal{B}_{\mathrm{Gr}_{G}} \star \mathrm{IC}_{-w_{0}(\check{\mu}), \operatorname{Gr}_{G}}[l(\check{\mu})]$. This complex is acyclic, since convolution with $\mathrm{IC}_{-w_{0}(\breve{\mu}), \mathrm{Gr}_{G}}$ is an exact functor.

We claim that for $\check{\mu}^{\prime}=\check{\mu}+\check{\nu}$ with $\check{\nu} \in \check{\Lambda}^{+}$we have a map of complexes

$$
\mathcal{B}_{\mathrm{Gr}_{G}} \star \mathrm{IC}_{-w_{0}(\check{\mu}), \mathrm{Gr}_{G}}[l(\check{\mu})] \rightarrow \mathcal{B}_{\mathrm{Gr}_{G}} \star \mathrm{IC}_{-w_{0}\left(\check{\mu}^{\prime}\right), \operatorname{Gr}_{G}}\left[l\left(\check{\mu}^{\prime}\right)\right] .
$$

For $w \cdot \check{\lambda} \in W_{a f f} / W$, the map

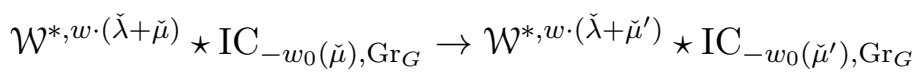

has been constructed in the definition of the inductive system that defines $\mathcal{M}^{w \cdot \check{\lambda}}$.

To check that this map respects the differential, we must show the following. Let $\tilde{w}=w \cdot \check{\lambda}$ and $\tilde{w}^{\prime}=w^{\prime} \cdot \check{\lambda}^{\prime}$ be such that $l\left(\tilde{w}^{\prime}\right)=l(\tilde{w})+1$, and the orbit $I \cdot \tilde{w}$ is in the closure of $I \cdot \tilde{w}^{\prime}$. Then we claim that for $\check{\mu} \in \check{\Lambda}^{+}$, the orbit $I \cdot(w \cdot(\check{\lambda}+\check{\mu}))$ is in the closure of $I \cdot\left(w \cdot\left(\check{\lambda}^{\prime}+\check{\mu}\right)\right)$, and the square

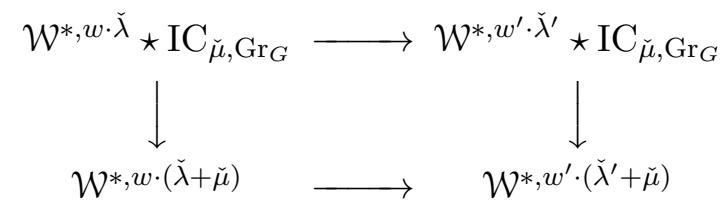

commutes, where the horizontal arrows are the canonical maps, corresponding to adjoining orbits.

Lemma 3.2.25. If $I \cdot \tilde{w} \subset \overline{I \cdot \tilde{w}^{\prime}}$, then as elements of $W_{a f f}, \tilde{w} \leq \tilde{w}^{\prime}$.

Proof. We need to show that $I \cdot \tilde{w}_{\mathrm{Fl}_{G}} \subset \overline{I \cdot \tilde{w}_{\mathrm{Fl}_{G}}^{\prime}}$, where the subscript $\mathrm{Fl}_{G}$ means that we are dealing with an orbit in $\mathrm{Fl}_{G}$ (vs. $\mathrm{Gr}_{G}$ ). 
Since the projection $\mathrm{Fl}_{G} \rightarrow \mathrm{Gr}_{G}$ is proper, there exists some $\tilde{w}_{1} \in W_{a f f}$, such that $\tilde{w}_{1}<\tilde{w}^{\prime}$, and $\tilde{w}_{1}=\tilde{w} \bmod W$. We have:

$$
l(\tilde{w}) \leq l\left(\tilde{w}_{1}\right) \leq l\left(\tilde{w}^{\prime}\right)-1
$$

Since $l(\tilde{w})=l\left(\tilde{w}^{\prime}\right)-1$, we obtain that $\tilde{w}_{1}=\tilde{w}$.

Note that by the lemma, we obtain that $w \cdot(\check{\lambda}+\check{\mu}) \leq w^{\prime} \cdot\left(\check{\lambda}^{\prime}+\check{\mu}\right)$, and hence we do have a containment

$$
I \cdot(w \cdot(\check{\lambda}+\check{\mu})) \subset \overline{I \cdot\left(w^{\prime} \cdot\left(\check{\lambda}^{\prime}+\check{\mu}\right)\right)} .
$$

Also, by the lemma, the map $\mathcal{W}^{*, w \cdot \check{\lambda}} \rightarrow \mathcal{W}^{*, w^{\prime} \cdot \check{\lambda}^{\prime}}$ is obtained from the map

$$
j_{*, w \cdot \check{\lambda}} \rightarrow j_{*, w^{\prime} \cdot \check{\lambda}^{\prime}}
$$

by convolving with $\delta_{1, \mathrm{Gr}_{G}}$. Note that the map $j_{*, w \cdot(\check{\lambda}+\check{\mu})} \rightarrow j_{*, w^{\prime} \cdot\left(\check{\lambda}^{\prime}+\check{\mu}\right)}$ is obtained from (25) by convolving on the right with $j_{*, \check{\mu}}$.

To prove the commutativity of the above diagram, it suffices to notice that the left vertical arrow is equal to the composition

$$
\begin{aligned}
& \mathcal{W}^{*, w \cdot \check{\lambda}} \star \mathrm{IC}_{\breve{\mu}, \mathrm{Gr}_{G}} \simeq j_{*, w \cdot \check{\lambda}} \star \mathrm{IC}_{\breve{\mu}, \mathrm{Gr}_{G}} \rightarrow j_{*, w \cdot \check{\lambda}} \star \mathcal{W}^{*, \check{\mu}} \simeq \\
& j_{*, w \cdot \check{\lambda}} \star j_{*, \check{\mu}} \star \delta_{1, \mathrm{Gr}_{G}} \simeq j_{*, w \cdot(\check{\lambda}+\check{\mu})} \star \delta_{1, \mathrm{Gr}_{G}} \simeq \mathcal{W}^{*, w \cdot(\check{\lambda}+\check{\mu})},
\end{aligned}
$$

and similarly for the right vertical arrow.

\section{Sheaves on SEMi-INFinite flags}

\subsection{Drinfeld's spaces and factorization.}

4.1.1. Let $X$ be a global curve. Let $\operatorname{Bun}_{G}$ denote the moduli stack of principal $G$ bundles on $X$. Let us recall the definition of the Drinfeld space $\overline{\operatorname{Bun}}_{N^{-}}{ }^{7}$

First we define a bigger space $\overline{\mathrm{Bun}}_{N^{-}}^{\prime}$ that classifies the data of a $G$-bundle $\mathcal{P}_{G}$ on $X$, and its generalized reduction to $N$, i.e., a collection of non-zero maps defined for each $\lambda \in \Lambda^{+}$

$$
\kappa^{\lambda}: V_{\mathcal{P}_{G}}^{\lambda} \rightarrow \mathcal{O}_{X},
$$

where $V^{\lambda}$ is the corresponding Weyl module over $G$, and $V_{\mathcal{P}_{G}}^{\lambda}$ is the associated vector bundle. The collection $\kappa^{\lambda}$ is required to satisfy the Plücker relations, cf. [FM, BG]. We will denote by $\mathfrak{p}$ the tautological projection $\overline{\operatorname{Bun}}_{N^{-}}^{\prime} \rightarrow$ Bun $_{G}$.

We have a natural action of $T$ on $\overline{\mathrm{Bun}}_{N^{-}}$: an element $t \in T$ multiplies each $\kappa^{\lambda}$ by $\lambda(t)$. It is easy to see that the map $\overline{\operatorname{Bun}}_{N^{-}}^{\prime} / T \rightarrow \operatorname{Bun}_{G}$ is proper.

If $[G, G]$ is simply-connected, then $\overline{\mathrm{Bun}}_{N^{-}}^{\prime}$ is the sought-for Drinfeld space $\overline{\mathrm{Bun}}_{N^{-}}$. Otherwise we proceed as follows.

Lemma 4.1.2. Let $G_{1} \rightarrow G_{2}$ be an isogeny, i.e., a homomorphism, whose kernel is contained in $Z\left(G_{1}\right)$ and whose image contains $\left[G_{2}, G_{2}\right]$. Then the natural map $\overline{\operatorname{Bun}}_{N^{-}}^{\prime}\left(G_{1}\right) \rightarrow \overline{\operatorname{Bun}}_{N^{-}}^{\prime}\left(G_{2}\right)$ is a closed embedding.

\footnotetext{
${ }^{7}$ The esposition in the section substantially relies on the results of $[\mathrm{BG}],[\mathrm{BFGM}]$ and $[\mathrm{FGV}]$, and certain familiarity with these papers will be assumed.
} 
Proof. First, it is easy to see that if we have a short exact sequence

$$
1 \rightarrow G^{\prime} \rightarrow G \rightarrow T^{\prime} \rightarrow 1
$$

where $T^{\prime}$ is a torus then $\overline{\operatorname{Bun}}_{N^{-}}^{\prime}\left(G^{\prime}\right) \rightarrow \overline{\operatorname{Bun}}_{N^{-}}^{\prime}(G)$ is an isomorphism. This reduces the assertion of the lemma to the case when $G_{1} \rightarrow G_{2}$ is surjective with finite kernel. Let $k$ be the index if $\check{\Lambda}_{1}$ in $\check{\Lambda}_{2}$.

Since each of $\overline{\operatorname{Bun}}_{N^{-}}^{\prime}\left(G_{i}\right) / T_{i}, i=1,2$ is proper over $\operatorname{Bun}_{G_{i}}$, the map

$$
\overline{\operatorname{Bun}}_{N^{-}}^{\prime}\left(G_{1}\right) / T_{1} \rightarrow \overline{\operatorname{Bun}}_{N^{-}}^{\prime}\left(G_{2}\right) / T_{2}
$$

is proper. Hence, it remains to see that the map $\overline{\operatorname{Bun}}_{N^{-}}^{\prime}\left(G_{1}\right) \rightarrow \overline{\operatorname{Bun}}_{N^{-}}^{\prime}\left(G_{2}\right)$ is injective on the level of $S$-points for any base $S$.

Let $\left(\mathcal{P}_{G_{2}},\left\{\kappa_{2}^{\lambda}\right\}\right)$ be an $S$-point of $\overline{\operatorname{Bun}}_{N^{-}}^{\prime}\left(G_{2}\right)$, and let $\left(\mathcal{P}_{G_{1}},\left\{\kappa_{1}^{\lambda}\right\}\right)$ be its lift to a point of $\overline{\operatorname{Bun}}_{N^{-}}^{\prime}\left(G_{1}\right)$. Then the image of $\kappa_{1}^{\lambda}$ in $V_{\mathcal{P}_{G_{1}}}^{\lambda}$ is fixed by the condition that

$$
\left(\kappa_{1}^{\lambda}\right)^{\otimes k}=\kappa_{2}^{k \cdot \lambda}: V_{\mathcal{P}_{G_{1}}}^{k \cdot \lambda} \simeq V_{\mathcal{P}_{G_{2}}}^{k \cdot \lambda} \rightarrow \mathcal{O}_{X}
$$

Hence, when $\mathcal{P}_{G_{1}}$ is fixed, any two choices of systems $\left\{\kappa_{1}^{\lambda}\right\}$ differ by an element of $T_{1,2}:=\operatorname{ker}\left(T_{1} \rightarrow T_{2}\right) \simeq \operatorname{ker}\left(G_{1} \rightarrow G_{2}\right)$. However, two such lifts are isomorphic as points of $\overline{\operatorname{Bun}}_{N^{-}}^{\prime}\left(G_{1}\right)$, via the automorphism of $\mathcal{P}_{G_{1}}$ given by the same element of $T_{1,2}$.

Finally, if $\mathcal{P}_{G_{1}}^{\prime}$ is another principal $G_{1}$-bundle that reduces to $G_{2}$, there exists a principal $T_{1,2}$-bundle $\mathcal{P}_{T_{1,2}}$, such that $\mathcal{P}_{G_{1}}^{\prime} \simeq \mathcal{P}_{G_{1}} \stackrel{T_{1,2}}{\times} \mathcal{P}_{T_{1,2}}$. Then for every $\lambda$ as above,

$$
V_{\mathcal{P}_{G_{1}}^{\prime}}^{\lambda} \simeq V_{\mathcal{P}_{G_{1}}}^{\lambda} \otimes \mathcal{P}_{T_{1,2}}^{\check{\lambda}},
$$

where $\mathcal{P}_{T_{1,2}}^{\check{\lambda}}$ is the line bundle associated with $\mathcal{P}_{T_{1,2}}$ and the character $\lambda$.

However, the data of $\kappa_{1}^{\lambda}$ for $V_{\mathcal{P}_{G_{1}}^{\prime}}^{\lambda}$ identifies the line sub-sheaf

$$
\left(\mathcal{P}_{T_{1,2}}^{\check{\check{C}}}\right)^{-1} \subset\left(V^{\lambda}\right)_{\mathcal{P}_{G_{1}}^{*}}^{*} \otimes\left(\mathcal{P}_{T_{1,2}}^{\check{\lambda}}\right)^{-1}
$$

with $\mathcal{O}_{X}$, thereby giving a trivialization of $\mathcal{P}_{T_{1,2}}$.

For an arbitrary group $G$ we can find a group $G^{\prime}$ with a surjective isogeny $G^{\prime} \rightarrow G$, such that (a) $\operatorname{ker}\left(G^{\prime} \rightarrow G\right)$ is connected, and (b) $\left[G^{\prime}, G^{\prime}\right]$ is simply connected.

We define $\overline{\operatorname{Bun}}_{N^{-}}$as the image of $\overline{\operatorname{Bun}}_{N^{-}}\left(G^{\prime}\right)=\overline{\operatorname{Bun}}_{N^{-}}^{\prime}\left(G^{\prime}\right)$ in $\overline{\operatorname{Bun}}_{N^{-}}^{\prime}$ under

$$
\overline{\operatorname{Bun}}_{N^{-}}^{\prime}\left(G^{\prime}\right) \rightarrow \overline{\operatorname{Bun}}_{N^{-}}^{\prime}(G) .
$$

By the above lemma, this is a closed substack of $\overline{\mathrm{Bun}}_{N^{-}}$, and it is easy to see that it does not depend on the choice of $G^{\prime}$. 
4.1.3. Variants. We fix a point $x \in X$. For a coweight $\check{\nu}$, let $\leq \check{\operatorname{Bun}^{-}}{\overline{N^{-}}}^{\prime}$ denote a version of $\overline{\operatorname{Bun}}_{N^{-}}^{\prime}$, where we allow each $\kappa^{\lambda}$ have a pole at $x$ of order $\leq\langle\lambda, \check{\nu}\rangle$.

For $G^{\prime}$ as above, due to the fact that the kernel of $G^{\prime} \rightarrow G$ is connected, we can find a preimage $\check{\nu}^{\prime}$ of $\check{\nu}$ in the coweight lattice $\check{\Lambda}^{\prime}$ of $G^{\prime}$, and we define $\sum_{\leq \check{\nu}} \overline{\mathrm{Bun}}_{N^{-}} \subset_{\leq \check{\nu}} \overline{\mathrm{Bun}}_{N^{-}}^{\prime}$ as the image of $\sum_{\llcorner} \overline{\operatorname{Bun}}_{N^{-}}^{\prime}\left(G^{\prime}\right)$ under

$$
\leq \check{\nu}^{\prime} \overline{\operatorname{Bun}}_{N^{-}}^{\prime}\left(G^{\prime}\right) \rightarrow \leq \check{\nu} \overline{\operatorname{Bun}}_{N^{-}}^{\prime} .
$$

As in Lemma 4.1.2 one shows that the map in (26) is a closed embedding. Moreover, its image is easily seen to be independent of the choice of $\check{\nu}^{\prime}$ for a fixed $G^{\prime}$, and of $G^{\prime}$ itself.

If $\check{\nu}_{1}-\check{\nu}_{2} \in \check{\Lambda}^{\text {pos }}$ we have a natural closed embedding $\leq \check{\nu}_{2} \overline{\operatorname{Bun}}_{N^{-}} \hookrightarrow \leq \check{\nu}_{1} \overline{\operatorname{Bun}}_{N^{-}}$. We define ${ }_{\infty} \overline{\operatorname{Bun}}_{N^{-}}$as

$$
\underset{\check{\nu} \in \check{\Lambda}}{\lim }\left(\leq \check{\nu} \overline{\operatorname{Bun}}_{N^{-}}\right)
$$

with respect to the natural ordering on $\check{\Lambda}$ and the above closed embeddings.

By definition, $\infty \overline{\mathrm{Bun}}_{N^{-}}$splits into connected components, numbered by the quotient of $\check{\Lambda}$ by the coroot lattice.

Let ${ }_{\check{\nu}} \overline{\mathrm{Bun}}_{N^{-}}^{\prime}$ is an open substack of $<_{\leq \nu} \overline{\mathrm{Bun}}_{N^{-}}^{\prime}$ corresponding to the condition that each $\kappa^{\lambda}$ has a pole of order exactly $\leq\langle\lambda, \check{\nu}\rangle$ at $x$. Set

$$
{ }_{\nu} \overline{\operatorname{Bun}}_{N^{-}}:={ }_{\nu} \overline{\operatorname{Bun}}_{N^{-}}^{\prime} \cap \leq \check{L} \overline{\operatorname{Bun}}_{N^{-}} .
$$

One easily shows that $\check{\nu}_{\nu} \overline{\operatorname{Bun}}_{N^{-}}$equals the image of $\check{\nu}^{\prime} \overline{\operatorname{Bun}}_{N^{-}}^{\prime}\left(G^{\prime}\right)$ under the map of (26).

Let us note that over each ${ }_{\nu} \overline{\mathrm{Bun}}_{N^{-}}$there exists a canonical $N^{-}[[t]]$-torsor, which we will denote by ${ }_{\check{\nu}} \mathcal{N}$. We will denote by ${ }_{\check{\nu}}^{k} \mathcal{N}$ the induced $N^{-}\left([t] / t^{k}\right)$-torsor.

We will denote by $\bar{i}_{\leq \check{\nu}}$ (resp., $\bar{i}_{\check{\nu}}$ ) the closed (resp., locally closed) embedding of ${ }_{\leq \check{\nu}} \overline{\mathrm{Bun}}_{N^{-}}\left(\right.$resp., $\left.{ }_{\check{\nu}} \overline{\mathrm{Bun}}_{N^{-}}{ }^{-}\right)$into ${ }_{\infty} \overline{\mathrm{Bun}}_{N^{-}}$. We have:

$$
{ }_{\check{\nu}} \overline{\mathrm{Bun}}_{N^{-}}={ }_{\leq \check{\nu}} \overline{\mathrm{Bun}}_{N^{-}}-{\underset{\check{\nu}}{1}<\check{\nu}}_{\check{\nu}_{1}} \overline{\mathrm{Bun}}_{N^{-}} \text {. }
$$

We let $\check{\nu} \operatorname{Bun}_{N^{-}}$denote the open sub-stack of $\bar{\nu}_{\check{\mathrm{Bun}}}{ }_{N^{-}}$, where we demand that the maps $\kappa^{\lambda}$ have no zeroes away from $x$. This substack is isomorphic to $\operatorname{Bun}_{B^{-}} \underset{\operatorname{Bun}_{T}}{\times} p t$, where the map $p t \rightarrow \operatorname{Bun}_{T}$ corresponds to the point $\mathcal{P}_{T}^{0}(\check{\nu} \cdot x)$. We will denote by $i_{\nu}$ the

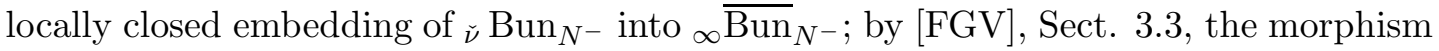
$i_{\nu}$ is affine.

Let $\bar{x}^{\prime}:=x_{1}^{\prime}, \ldots, x_{m}^{\prime}$ be a collection of points on $X$, distinct from $x$. Let $\infty \overline{\operatorname{Bun}}_{N^{-}}^{\text {n.z. } \bar{x}^{\prime}}$ be the open sub-stack of ${ }_{\infty} \overline{\mathrm{Bun}}_{N^{-}}$defined by the condition that the maps $\kappa^{\lambda}$ have no zeroes at $x_{1}^{\prime}, \ldots, x_{m}^{\prime}$. As in [FGV], Sect. 3.2, one shows that over ${ }_{\infty} \overline{\operatorname{Bun}}_{N^{-}}^{\text {n.z. } \bar{x}^{\prime}}$ there exists a natural torsor with respect to the group-scheme $\prod_{j=1, \ldots, m} N^{-}\left[\left[t_{j}^{\prime}\right]\right]$, denoted $\mathcal{N} \bar{x}^{\prime}$, where $t_{j}^{\prime}$ is a local coordinate at $x_{j}^{\prime}$. Moreover, $\mathcal{N}^{\bar{x}^{\prime}}$ carries an action of the group-indscheme $\prod_{j=1, \ldots, m} N^{-}\left(\left(t_{j}^{\prime}\right)\right)$. 
For an integer $k$ let ${ }^{k} \operatorname{Bun}_{G}$ denote the principal $G^{k}$-bundle over $\operatorname{Bun}_{G}$ corresponding to choosing a structure of level $k$ at $x$ in a $G$-bundle. We will denote by ${ }_{\infty}^{k} \overline{\operatorname{Bun}}_{N^{-}}$the Cartesian product $\infty \overline{\operatorname{Bun}}_{N^{-}} \underset{\operatorname{Bun}_{G}}{\times}{ }^{k} \operatorname{Bun}_{G}$.

We will denote by ${ }_{\leq \check{\nu}}^{k} \overline{\operatorname{Bun}}_{N^{-}}, \underset{\nu}{k} \overline{\operatorname{Bun}}_{N^{-}},{ }_{\check{\nu}}^{k} \operatorname{Bun}_{N^{-}}$the corresponding stacks obtained by base change. By a slight abuse of notation, we will use the symbols $\bar{i}_{\leq \check{\nu}}, \bar{i}_{\check{\nu}}$ and $i_{\check{\nu}}$ for the embeddings of these stacks into ${ }_{\infty}^{k} \overline{\operatorname{Bun}}_{N^{-}}$. Similary, we introduce the stacks

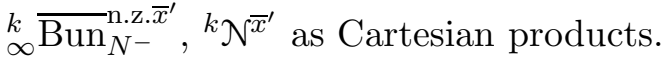

Note that there is a natural isomorphism

$$
{ }_{\nu}^{k} \overline{\operatorname{Bun}}_{N^{-}} \simeq G\left([t] / t^{k}\right) \stackrel{N^{-}\left([t] / t^{k}\right)}{\times} \underset{\nu}{k} \mathcal{N} .
$$

In particular, we obtain a natural map

$$
\overline{\mathrm{ev}}_{\check{\nu}}:{ }_{\check{\nu}}^{k} \overline{\mathrm{Bun}}_{N^{-}} \rightarrow\left(G / N^{-}\right)\left([t] / t^{k}\right) \rightarrow\left(G / B^{-}\right)\left([t] / t^{k}\right) .
$$

The restriction of this map to $\underset{\check{\nu}}{k} \operatorname{Bun}_{N}$, denoted $\mathrm{ev}_{\check{\nu}}$, is smooth.

4.1.4. For $\check{\mu} \in \check{\Lambda}$ let $\operatorname{Bun}_{B}^{\check{\mu}}$ be the corresponding connected component of $\operatorname{Bun}_{B}$. We recall that $\operatorname{Bun}_{B}^{\breve{\mu}}$ can be interpreted as the stack classifying the data of a principal $G$ bundle $\mathcal{P}_{G}$ on $X$, a $T$-bundle $\mathcal{P}_{T}$, such that each associated line bundle $\mathcal{P}_{T}^{\lambda}$ has degree $-\langle\lambda, \check{\mu}\rangle$, and a collection of bundle maps

$$
\kappa^{\lambda,-}: \mathcal{P}_{T}^{\lambda} \rightarrow V_{\mathcal{P}_{G}}^{\lambda}
$$

defined for $\lambda \in \Lambda^{+}$, which satisfy the Plücker relations. (Here $\mathcal{P}_{T}^{\lambda}$ denoted the line bundle associated with $\mathcal{P}_{T}$ and the character $\lambda: T \rightarrow \mathbb{G}_{m}$.)

Note that if $\check{\mu}$ is such that $\langle\alpha, \check{\mu}\rangle>(2 g-2)$ for all positive roots $\alpha$, then the map $\mathfrak{p}^{\check{\mu},-}: \operatorname{Bun}_{B^{-}}^{\check{\mu}} \rightarrow \operatorname{Bun}_{G}$ is smooth.

Consider the Cartesian product $\infty \overline{\operatorname{Bun}}_{N^{-}} \underset{\operatorname{Bun}_{G}}{\times} \operatorname{Bun}_{B}^{\check{\mu}}$. We will denote by $\infty z^{\check{\mu}}$ the corresponding Zastava space, i.e. the open substack of the above Cartesian product, defined by the condition that the reductions to $N^{-}$and $B$ are transversal at the generic point of the curve. This means that the composed maps

$$
\mathcal{P}_{T}^{\lambda} \stackrel{\kappa^{\lambda,-}}{\longrightarrow} V_{\mathcal{P}_{G}}^{\lambda} \stackrel{\kappa^{\lambda}}{\longrightarrow} \mathcal{O}_{X}
$$

are non-zero for all $\lambda \in \Lambda^{+}$.

We will denote by ${ }_{\infty}^{k} z^{\check{\mu}}$ the stack obtained by adding a structure of level $k$ to the $G$-bundle $\mathcal{P}_{G}$ at $x$. All of the above stacks are acted on by the group $T$.

Let us denote by ${ }_{\leq \check{\nu}}^{k} z^{\check{\mu}}$ (resp., ${ }_{\check{\nu}}^{k} z^{\check{\mu}},{ }^{k} z^{\check{\mu}}$ ) the preimage in ${ }_{\infty}^{k} z^{\check{\mu}}$ of the substack ${ }_{\leq \check{\nu}}^{k} \overline{\operatorname{Bun}}_{N^{-}}$(resp., ${ }_{\check{\nu}}^{k} \overline{\operatorname{Bun}}_{N^{-}},{ }^{k} \overline{\operatorname{Bun}}_{N^{-}}={ }_{\leq 0}^{k} \overline{\operatorname{Bun}}_{N^{-}}$) of ${ }_{\infty}^{k} \overline{\operatorname{Bun}}_{N^{-}}$. Note that ${ }_{\leq \check{\nu}} z^{\check{\mu}}$ is empty unless $\check{\nu}+\check{\mu} \in \check{\Lambda}^{\text {pos }}$. By $\underset{\nu}{k} \underset{\nu}{\stackrel{\circ}{\alpha}}$ we will denote the open substack of $\underset{\nu}{k} z^{\check{\mu}}$ equal to the preimage of $\underset{\check{\nu}}{k} \operatorname{Bun}_{N^{-}}$.

For $\check{\mu} \in \check{\Lambda}^{\text {pos }}$, let $X^{\check{\mu}}$ be the corresponding partially symmetrized power of the curve. By definition, $X^{\check{\mu}}$ classifies the data of a principal $T$-bundle $\mathcal{P}_{T}$ and its generic 
trivialization, such that for $\lambda \in \Lambda^{+}$the resulting maps $\mathcal{P}_{T}^{\lambda} \rightarrow \mathcal{O}_{X}$ are all regular and the divisor of zeroes has degree $\langle\lambda, \breve{\mu}\rangle$.

For $\check{\nu} \in \check{\Lambda}$, let $\leq \check{\nu} X^{\check{\mu}}$ be a version of $X^{\check{\mu}}$, where the maps $\mathcal{P}_{T}^{\lambda} \rightarrow \mathcal{O}_{X}$ are allowed to have poles at $x$ of order $\leq\langle\lambda, \check{\nu}\rangle$ for $\lambda \in \Lambda^{+}$. This space is empty unless $\check{\mu}+\check{\nu} \in \check{\Lambda}^{\text {pos }}$. If $\check{\nu}_{1}-\check{\nu}_{2} \in \Lambda^{\text {pos }}$ we have a natural closed embedding

$$
\leq \check{\nu}_{2} X^{\check{\mu}} \hookrightarrow \check{\nu}_{2} X^{\check{\mu}}
$$

We define ${ }_{\infty} X^{\check{\mu}}$ as the ind-scheme

$$
\infty^{\check{\mu}}=\underset{\check{\nu} \in \check{\Lambda}}{\lim _{\breve{\Lambda}}}\left(\leq \check{\nu} X^{\check{\mu}}\right)
$$

with respect to the usual ordering on $\check{\Lambda}$ and the above closed embeddings. This space also splits into connected components numbered by the quotient of $\check{\Lambda}$ by the coroot lattice.

By construction, we have a natural map

$$
\infty \mathfrak{s}^{\check{\mu}}: \infty Z^{\check{\mu}} \rightarrow \infty X^{\check{\mu}} \text {. }
$$

We will denote the restriction of $\infty_{\mathfrak{s}^{\check{\mu}}}$ to $\leq \check{\nu} Z^{\check{\mu}}$ (resp., $\left.Z^{\check{\mu}}={ }_{\leq 0} z^{\check{\mu}}\right)$ by $\leq_{\nu} \mathfrak{s}^{\check{\mu}}\left(\right.$ resp., $\left.\mathfrak{s}^{\check{\mu}}\right)$. Note that $\leq \check{\nu} \mathfrak{s}^{\check{\mu}}$ maps to $<\check{\nu} X^{\check{\mu}}$.

We will denote by ${ }^{k} \mathfrak{s}^{\check{\mu}}$ the composition of $\mathfrak{s}^{\check{\mu}}$ and the forgetful map ${ }_{\infty}^{k} z^{\check{\mu}} \rightarrow \infty Z^{\check{\mu}}$, and similarly for ${ }_{\leq \check{\nu}}^{k} \mathfrak{s}^{\check{\mu}}, k_{\mathfrak{s}^{\check{\mu}}}$.

Let $\stackrel{\circ}{X}$ denote the open curve $X-x$, and $\stackrel{\circ}{X}$ 崩 be the corresponding open subset of $X^{\check{\mu}}$. For $\check{\mu}_{1}, \check{\mu}_{2}$ we will denote by $\left(\stackrel{\circ}{X}^{\check{\mu}_{1}} \times_{\infty} X^{\check{\mu}_{2}}\right)_{d i s j}$ the open subset in the product $\stackrel{\circ}{X^{\mu_{1}}} \times_{\infty} X^{\check{\mu}_{2}}$, corresponding to the condition that the two divisors have disjoint support.

As in $[\mathrm{BFGM}]$, we have:

Lemma 4.1.5. For $\check{\mu}_{1}+\check{\mu}_{2}=\check{\mu}$ there exist natural isomorphisms

$$
{ }_{\infty}^{k} z^{\check{\mu}} \underset{\infty X^{\check{\mu}}}{\times}\left(\stackrel{\circ}{X^{\check{\mu}}} \times_{\infty} X^{\check{\mu}_{2}}\right)_{d i s j} \simeq\left(z^{\check{\mu}_{1}} \times_{\infty}^{k} z^{\check{\mu}_{2}}\right)_{X^{\check{\mu}_{1}} \times_{\infty} X^{\check{\mu}_{2}}}\left(\stackrel{\circ}{X}^{\check{\mu}_{1}} \times_{\infty} X^{\check{\mu}_{2}}\right)_{d i s j} .
$$

Let $x^{\prime}$ be any point of the curve, and for $\check{\mu} \in \check{\Lambda}^{\text {pos }}$, let $\check{\mu} \cdot x^{\prime}$ be the corresponding element of $\stackrel{\circ}{X^{\check{\mu}}}$. Then, by [BFGM], we have:

$$
\left(\mathfrak{s}^{\check{\mu}}\right)^{-1}\left(\check{\mu} \cdot x^{\prime}\right) \simeq\left(N\left(\left(t^{\prime}\right)\right) \cdot \check{\mu}\right) \cap \overline{\left(N^{-}\left(\left(t^{\prime}\right)\right) \cdot 1_{\mathrm{Gr}_{G}}\right)},
$$

where $t^{\prime}$ is a local coordinate at $x^{\prime}$.

In the same way we obtain that for an arbitrary element $\check{\mu} \in \check{\Lambda}$ and the point $\check{\mu} \cdot x \in \in_{\infty} X^{\check{\mu}}$

$$
{ }_{\infty}^{k} \mathfrak{F}^{\check{\mu}}:=\left(\begin{array}{l}
k \\
\infty
\end{array} \mathfrak{s}^{\check{\mu}}\right)^{-1}(\check{\mu} \cdot x) \simeq(N((t)) \cdot \check{\mu}) \underset{\mathrm{Gr}_{G}}{\times} G((t)) / G^{k}
$$


4.1.6. For an integer $m$, let $\operatorname{Jets}^{+}(T)^{m}$ be the group-scheme over $X^{(m)}$, whose fiber over a divisor $\Sigma m_{j} \cdot x_{j}$, where the points $x_{j}$ are distinct, is $\prod_{j} T\left[\left[t_{j}^{\prime}\right]\right]$. More precisely, for a test-scheme $S$ and an $S$-point $\varphi$ of $X^{(m)}$ its lift to an $S$-point of $\operatorname{Jets}^{+}(T)^{m}$ is an $X^{(m)}$-map

$$
\widehat{\Gamma}_{\varphi} \rightarrow T
$$

where $\widehat{\Gamma}_{\varphi} \subset S \times X$ is the formal neighbourhood of the preimage $\Gamma_{\varphi}$ of the incidence divisor in $X^{(m)} \times X$ under $\varphi \times$ id.

If $\check{\mu}, \check{\nu} \in \check{\Lambda}$ are two elements with $\check{\mu}+\check{\nu} \in \check{\Lambda}^{\text {pos }}$, we have a natural map $\leq \check{\nu} X^{\check{\mu}} \rightarrow X^{(m)}$, where $m=l(\check{\mu}+\check{\nu})$, and let $\leq \check{\nu} \operatorname{Jets}^{+}(T)^{\check{\mu}}$ be the resulting group-scheme on $\leq \check{\nu} X^{\check{\mu}}$.

Proposition-Construction 4.1.7. The group-scheme $\leq \check{\nu} \operatorname{Jets}^{+}(T)^{\check{\mu}}$ acts naturally on $\leq \check{\nu} Z^{\check{\mu}}$.

Proof. To simplify the notation, we will assume that $\check{\nu}=0$, and we will work with the "usual" Zastava space Z Ž

According to [BFGM], Sect. 2, given an $S$-point of $Z^{\check{\mu}}$, the resulting $G$-bundle $\mathcal{P}_{G}$ on $S \times X$ acquires a trivialization on $S \times X-\Gamma_{\varphi}$, where $\varphi$ is the composition of the initial map to $Z^{\check{\mu}}$ and

$$
z^{\check{\mu}} \rightarrow X^{\check{\mu}} \rightarrow X^{(m)} .
$$

As usual in this situation, given a map $g_{S}: \widehat{\Gamma}_{\varphi} \rightarrow G$, we can produce a new $G$-bundle $\mathcal{P}_{G}^{\prime}$, by declaring it to be the same as $\mathcal{P}_{G}$ on $S \times X-\Gamma_{\varphi}$ and $\widehat{\Gamma}_{\varphi}$ and changing the gluing data on the formal punctured neighbourhood of $\Gamma_{\varphi}$ by means of $g_{S}$.

If $g_{S}$ was a map $\widehat{\Gamma}_{\varphi} \rightarrow T$, then the data of $\kappa^{\lambda}$ and $\kappa^{\lambda,-}$ for $\mathcal{P}_{G}$ give rise to well-defined data of $\left(\kappa^{\lambda}\right)^{\prime}$ and $\left(\kappa^{\lambda,-}\right)^{\prime}$ for $\mathcal{P}_{G}^{\prime}$. Thus, we obtain a new point of $z^{\check{\mu}}$.

Note that $\leq \check{\nu} \operatorname{Jets}^{+}(T)^{\check{\mu}}$ contains as a direct factor the constant group-subscheme with fiber $T$. Its action on $\leq \check{\nu} Z^{\check{\mu}}$ coincides with the "global" one, mentioned above.

Let us consider now $k_{\leq \check{\nu}}^{k} z^{\check{\mu}}$. One can show that the above action of $\leq_{\check{\nu}} \operatorname{Jets}^{+}(T)^{\check{\mu}}$ on $\leq \check{\nu} z^{\check{\mu}}$ does not lift to an action of $k_{\leq \check{\nu}} z^{\breve{\mu}}$. However, we do have an action fiber-wise over each point of $\leq \check{\nu} X^{\check{\mu}}$. For example, the action of $T[[t]]$ on ${ }_{\breve{\mu}}^{k} \mathfrak{F}^{\check{\mu}}$ is given in terms of isomorphism

$$
\underset{\check{\mu}}{k} \mathfrak{F}^{\check{\mu}} \simeq\left(N((t)) \cdot \check{\mu} \cap \overline{\left(N^{-}\left(\left(t^{\prime}\right)\right) \cdot(-\check{\nu})\right)}\right) \underset{\mathrm{Gr}_{G}}{\times} G((t)) / G^{k}
$$

by the natural action of $T((t))$ on $G((t))$ by left multiplication.

We will use the following construction. Let us choose an identification $T \simeq \mathbb{G}_{m}^{r}$, and a point $y \in X-x$. For a string of positive integers $\bar{m}=m_{1}, \ldots, m_{r}$, consider the affine space consisting of $r$-tuples of functions $(X-y) \rightarrow \mathbb{A}^{1}$, whose values at $x$ is 1 , and the pole of the $i$-th function at $y$ is of order $\leq m_{i}$. We will denote this space by $\operatorname{Maps}(X, T)^{\bar{m}}$. 
The Abel-Jacobi map gives rise to a morphism $\operatorname{Maps}(X, T)^{\bar{m}} \rightarrow \stackrel{\circ}{X^{\bar{m}}}:=\prod_{i} \stackrel{\circ}{X^{\left(m_{i}\right)}}$, and we have a natural morphism

$$
\left(\operatorname{Maps}(X, T)^{\bar{m}} \times X\right) \underset{\stackrel{\circ}{X}^{\bar{m}} \times X}{\times}\left(\stackrel{\circ}{\left.X^{\bar{m}} \times X\right)_{d i s j}} \rightarrow T\right.
$$

where $\left(\stackrel{\circ}{X^{\bar{m}}} \times X\right)_{d i s j} \subset \stackrel{\circ}{X^{\bar{m}}} \times X$ has the same meaning as before-the complement to the incidence divisor. (This morphism explains the notation $\operatorname{Maps}(X, T)^{\bar{m}}$ for the above scheme.)

Proposition-Construction 4.1.8. We have a natural map

$$
\operatorname{act}_{T}:\left(\operatorname{Maps}(X, T)^{\bar{m}} \times_{\leq \check{\nu}}^{k} z^{\check{\mu}}\right)_{\check{X}^{\bar{m}} \times_{\leq \check{\nu}} X_{\check{\mu}}}^{\times}\left(\stackrel{\circ}{X}^{\bar{m}} \times_{\leq \check{\nu}} X^{\check{\mu}}\right)_{d i s j} \rightarrow{ }_{\leq \check{\nu}}^{k} z^{\check{\mu}} .
$$

Proof. We retain the notation from the proof of the previous proposition-construction. The difference now is that the map $g_{S}$ is defined on a Zarisky-open of $S \times X$ that contains $\Gamma_{\varphi}$ and $S \times x$. In particular, the restrictions of $\mathcal{P}_{G}$ and $\mathcal{P}_{G}^{\prime}$ to the formal neighborhood of $x$ are identified. Hence, $\mathcal{P}_{G}^{\prime}$ is also equipped with a structure of level $k$ at $x$.

\subsection{A category of perverse sheaves.}

4.2.1. For an integer $k$ we define the category $\operatorname{Perv}\left(\mathcal{F} l^{\frac{\infty}{2}}\right)^{G^{k}}$ to be the full subcategory of the category of $T$-equivariant perverse sheaves on ${ }_{\infty}^{k} \overline{\mathrm{Bun}}_{N^{-}}$, consisting of objects satisfying the following three properties:

(1) For a finite collection $\bar{x}=x_{1}^{\prime}, \ldots, x_{m}^{\prime}$ of points on $X$ distinct from $x$, the pull-back of $\mathcal{F}$ to ${ }^{k} \mathcal{N}^{\bar{x}^{\prime}}$ is equivariant with respect to the group-indscheme $\underset{j=1, \ldots, m}{\prod} N^{-}\left(\left(t_{j}^{\prime}\right)\right)$.

(2) The factorization property:

We say that a perverse sheaf $\mathcal{F}$ on ${ }_{\infty}^{k} \overline{\operatorname{Bun}}_{N^{-}}$is factorizable if for any $\check{\mu}_{1}, \check{\mu}_{2}$, satisfying $\left\langle\alpha, \check{\mu}_{i}\right\rangle>(2 g-2)$ and $\check{\mu}_{2}-\check{\mu}_{1} \in \check{\Lambda}^{\text {pos }}$, the retsriction of the pull-back $\mathfrak{p}^{-, \breve{\mu}_{2} *}(\mathcal{F})$ onto the left-hand side of

${ }_{\infty}^{k} z^{\check{\mu}_{2}} \underset{\infty}{\times X^{\check{\mu}_{2}}}\left(\stackrel{\circ}{X^{\check{\mu}_{2}-\check{\mu}_{1}}} \times_{\infty} X^{\check{\mu}_{1}}\right)_{d i s j} \simeq\left(z^{\check{\mu}_{2}-\check{\mu}_{1}} \times_{\infty}^{k} z^{\check{\mu}_{1}}\right)_{X^{\check{\mu}_{2}-\check{\mu}_{1}} \times_{\infty} X^{\check{\mu}_{1}}}\left(\stackrel{\circ}{X^{\check{\mu}_{2}}-\check{\mu}_{1}} \times_{\infty} X^{\check{\mu}_{1}}\right)_{d i s j}$

is isomorphic (up to a cohomological shift by the corresponding relative dimensions) to the restriction onto the right-hand side of the external product

$$
\mathrm{IC}_{Z^{\check{\mu}_{2}-\check{\mu}_{1}}} \otimes \mathfrak{p}^{-, \check{\mu}_{1} *}(\mathcal{F}) .
$$

(Note that both complexes in question are perverse sheaves, since the maps $\mathfrak{p}^{-, \breve{\mu}_{i}}$, $i=1,2$ are smooth by assumption.)

(3) If $\mathcal{F}$ is supported on ${ }_{\leq \check{\nu}}^{k} \overline{\operatorname{Bun}}_{N^{-}}$, then for $\check{\mu} \in \Lambda$, satisfying $\langle\alpha, \check{\mu}\rangle>(2 g-2)$, the pull-back of $\mathcal{F}$ on ${ }_{\leq \check{\nu}}^{k} z^{\check{\mu}}$ is $\operatorname{Maps}(X, T)^{\bar{m}}$-equivariant for any $\bar{m}$. The latter means that 
there exists an isomorphism between two pull-backs of $\left.\left(\mathfrak{p}^{\check{\mu},-}\right)^{*}(\mathcal{F})\right|_{\leq \check{\nu}} z^{\check{\mu}}$ to

$$
\left(\operatorname{Maps}(X, T)^{\bar{m}} \times \underset{\leq \check{\nu}}{k} z^{\check{\mu}}\right)_{\stackrel{\circ}{X}^{\bar{m}} \times_{\leq \check{\nu}} X^{\check{\mu}}}^{\times}\left(\stackrel{\circ}{X}^{\bar{m}} \times_{\leq \check{\nu}} X^{\check{\mu}}\right)_{d i s j},
$$

which induces the identity map on the further restriction of both sides to the unit point of $\operatorname{Maps}(X, T)^{\bar{m}}$.

Remark. As we shall see, imposing property (1) is in fact superfluous, i.e., it follows formally from the factorization property (2). In addition, some portion of property (2) follows from (1).

In addition, if $k=1$ (which the main case of interest for this paper), property (3) follows automatically. ${ }^{8}$

In general, we shall see that property (3) is equivalent to imposing the condition that either $*_{-}$or !-restriction of $\left.\mathfrak{p}^{\check{\mu},-}(\mathcal{F})\right|_{\leq \check{\nu}} z_{\breve{\mu}}$ to $\underset{\breve{\mu}}{k} \mathfrak{F}^{\check{\mu}}$ is $T[[t]]$-equivariant.

In the sequel we will formulate a conjecture, from which it follows that the category $\operatorname{Perv}\left(\mathcal{F} l^{\frac{\infty}{2}}\right)^{G^{k}}$ is independent of the curve $X$, and possesses the symmetries expected from "the category of $G^{k}$-equivariant sheaves on $\mathcal{F} l^{\frac{\infty}{2}}:=G((t)) / N^{-}((t)) \cdot T[[t]]$ ", in particular, it will carry an action of the lattice $\check{\Lambda} \simeq T((t)) / T[[t]]$ by translation functors.

4.2.2. Our present goal is to describe the irreducibles in $\operatorname{Perv}\left(\mathcal{F} l^{\frac{\infty}{2}}\right)^{G^{k}}$. Recall the isomorphism (27), which realizes ${ }_{\nu}^{k} \overline{\mathrm{Bun}}_{N^{-}}$as a fibration over the base $G / N^{-}\left([t] / t^{k}\right)$ with typical fiber ${ }_{\check{\nu}}^{k} \mathcal{N}$. (In fact, ${ }_{\check{\nu}}^{k} \overline{\mathrm{Bun}}_{N^{-}}$is a principal $N^{-}\left([t] / t^{k}\right)$-bundle over the product $G / N^{-}\left([t] / t^{k}\right) \times{ }_{\check{\nu}} \overline{\mathrm{Bun}}_{N^{-}}$.)

In particular, for a perverse sheaf $\mathcal{F}^{\prime}$ on $\left(G / N^{-}\right)\left([t] / t^{k}\right)$, we can form the twisted external product

$$
\mathcal{F}^{\prime} \widetilde{\otimes} \mathrm{IC}_{\check{\nu}} \mathcal{N} \in \operatorname{Perv}\left({ }_{\check{\nu}}^{k} \overline{\operatorname{Bun}}_{N^{-}}\right) .
$$

Up to a cohomological shift, it is isomorphic to the pull-back of

$$
\mathcal{F}^{\prime} \otimes \mathrm{IC}_{\check{\nu}} \overline{\operatorname{Bun}}_{N^{-}} \in \operatorname{Perv}\left(G / N^{-}\left([t] / t^{k}\right) \times{ }_{\check{\nu}} \overline{\operatorname{Bun}}_{N^{-}}\right) .
$$

\section{Proposition 4.2.3.}

(1) For $\mathcal{F} \in \operatorname{Perv}\left(\mathcal{F} l^{\frac{\infty}{2}}\right)^{G^{k}}$, all perverse cohomologies of the restriction $\bar{i}_{\check{\nu}}^{*}(\mathcal{F})$ are of the form $\mathcal{F}^{\prime} \widetilde{\otimes} \mathrm{IC}_{\tilde{\nu}} \mathfrak{N}$, where $\mathcal{F}^{\prime}$ is a perverse sheaf on $\left(G / N^{-}\right)\left([t] / t^{k}\right)$, that comes as a pull-back from a perverse sheaf on $\left(G / B^{-}\right)\left([t] / t^{k}\right)$.

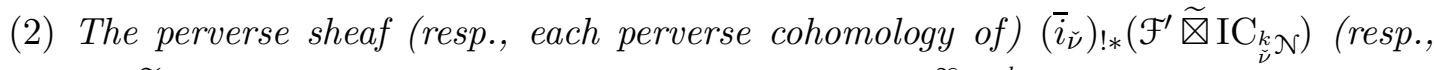
$\left(\bar{i}_{\check{\nu}}\right) !\left(\mathcal{F}^{\prime} \widetilde{\otimes} \mathrm{IC}_{\check{\nu}} \mathcal{N}\right)$ ) for $\mathcal{F}^{\prime}$ as above is an object of $\operatorname{Perv}\left(\mathcal{F} l^{\frac{\infty}{2}}\right)^{G^{k}}$.

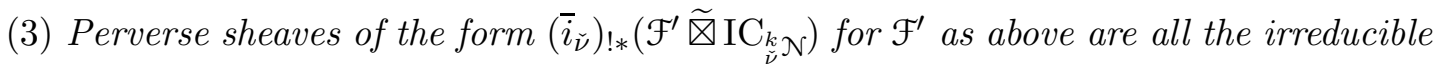
objects of $\operatorname{Perv}\left(\mathcal{F} l^{\frac{\infty}{2}}\right)^{k}$.

\footnotetext{
${ }^{8}$ We remark also that property (3) has to do with the fact that our category $\operatorname{Perv}\left(\mathcal{F} l^{\frac{\infty}{2}}\right)^{G^{k}}$ models perverse sheaves on $G((t)) / N^{-}((t)) \cdot T[[t]]$ rather than on $G((t)) / N^{-}((t))$.
} 
The rest of this subsection is devoted to the proof of the proposition. Note, however, that point (3) is a formal corollary of points (1) and (2).

The factorization isomorphisms of Lemma 4.1 .5 respect the substacks ${ }_{\check{\nu}}^{k} \check{z}^{\check{\mu}}, \underset{\nu}{k} z^{\check{\mu}},{ }_{\leq}^{k} z^{\check{\mu}}$ of ${ }_{\infty}^{k} z^{\check{\mu}}$. Hence, it makes sense to introduce the category ${ }_{\check{\nu}}^{\prime} \operatorname{Perv}\left(\mathcal{F} l^{\frac{\infty}{2}}\right)^{G^{k}}$, which is a full subcategory of Perv $\left({ }_{\check{\nu}}^{k} \overline{\mathrm{Bun}}_{N^{-}}\right)$, consisting of objects, satisfying the same conditions (1), (2) and (3) as in the definition of $\operatorname{Perv}\left(\mathcal{F} l^{\frac{\infty}{2}}\right)^{G^{k}}$.

It is clear that for $\mathcal{F}_{1} \in \operatorname{Perv}\left(\mathcal{F}^{\frac{\infty}{2}}\right)^{G^{k}}$, the perverse cohomologies of the restriction $\bar{i}_{\check{\nu}}^{*}\left(\mathcal{F}_{1}\right)$ are objects of ${ }_{\check{\nu}}^{\prime} \operatorname{Perv}\left(\mathcal{F} l^{\frac{\infty}{2}}\right)^{G^{k}}$, and vice versa: for $\mathcal{F}_{2} \in{ }_{\nu}^{\prime} \operatorname{Perv}\left(\mathcal{F l} l^{\frac{\infty}{2}}\right)^{G^{k}}$, the perverse sheaf (resp., each perverse cohomology of) $\left(\bar{i}_{\breve{\nu}}\right)_{! *}\left(\mathcal{F}_{2}\right)$ (resp., $\left(\bar{i}_{\breve{\nu}}\right)_{!}\left(\mathcal{F}_{2}^{\prime}\right)$ ) belongs to $\operatorname{Perv}\left(\mathcal{F} l^{\frac{\infty}{2}}\right)^{G^{k}}$.

Therefore, the assertion of the proposition reduces to showing that the functor $\mathcal{F}^{\prime} \mapsto$ $\mathcal{F}^{\prime} \widetilde{\otimes} \mathrm{IC}_{\check{\nu}} \overline{\mathrm{Bun}}_{N^{-}}$defines an equivalence

$$
\operatorname{Perv}\left(\left(G / B^{-}\right)\left([t] / t^{k}\right)\right) \rightarrow{ }_{\nu}^{\prime} \operatorname{Perv}\left(\mathcal{F} l^{\frac{\infty}{2}}\right)^{G^{k}}
$$

First, we claim that every object of ${ }_{\nu} \operatorname{Perv}\left(\mathcal{F} l^{\frac{\infty}{2}}\right)^{G^{k}}$ is the Goresky-MacPherson extension of its restriction to the open sub-stack $\check{\nu} \operatorname{Bun}_{N}$. Indeed, if it were not, we would be able to find $\check{\mu}_{1}$ and $\check{\mu}_{2}$ large enough, so that either ! or *-restriction of $\mathcal{F}$ to the closed sub-stack

$$
\left(\left(z^{\check{\mu}_{1}}-\stackrel{\circ}{\check{Z}^{\check{\mu}_{1}}}\right) \times_{\infty}^{k} z^{\check{\mu}_{2}}\right)_{X^{\check{\mu}_{1}} \times_{\infty} X^{\check{\mu}_{1}}}\left(\stackrel{\circ}{X^{\check{\mu}_{2}}} \times_{\infty} X^{\check{\mu}_{2}}\right)_{d i s j}
$$

would have non-zero perverse cohomologies in positive (resp., negative) degrees. However, this contradicts the factorizability property (2).

Let us denote by $\check{\nu}_{\nu} \operatorname{Perv}\left(\mathcal{F} l^{\frac{\infty}{2}}\right) G^{k}$ the corresponding full subcategory of Perv $\left({ }_{\check{\nu}}^{k} \operatorname{Bun}_{N^{-}}\right)$ consisting of perverse sheaves, satisfying (1) and (3). We are reduced to showing that

$$
\mathcal{F}^{\prime} \mapsto \mathcal{F}^{\prime} \widetilde{\otimes} \mathrm{IC}_{\check{\nu} \mathcal{N}^{k}}: \operatorname{Perv}\left(\left(G / B^{-}\right)\left([t] / t^{k}\right)\right) \rightarrow{ }_{\nu} \operatorname{Perv}\left(\mathcal{F l} l^{\frac{\infty}{2}}\right)^{G^{k}}
$$

is an equivalence. Note that the latter functor is isomorphic, up to a cohomological shift, to the pull-back functor under the smooth map

$$
\underset{\check{\nu}}{k} \operatorname{Bun}_{N^{-}} \stackrel{\mathrm{ev}_{\check{\breve{\nu}}}}{\longrightarrow}\left(G / N^{-}\right)\left([t] / t^{k}\right) \rightarrow\left(G / B^{-}\right)\left([t] / t^{k}\right) .
$$

The fact that the functor in question is fully faithful is clear, since the map in (30) has connected fibers. Hence, it remains to show the essential surjectivity.

First, let us show that any $\mathcal{F} \in \operatorname{Perv}\left(\underset{\check{\nu}}{k} \operatorname{Bun}_{N^{-}}\right)$is the pull-back under ev $\check{\check{\nu}}$ of some perverse sheaf $\mathcal{F}^{\prime}$ on $(G / N)\left([t] / t^{k}\right)$. 
For any non-empty collection of points $\bar{x}^{\prime}$, distinct from $x$, consider the pull-back of $\mathcal{F}$ to ${ }_{\breve{\nu}}^{k} \operatorname{Bun}_{N^{-}}{ }_{\breve{\nu}}^{k} \frac{\times}{\operatorname{Bun}_{N^{-}}}{ }^{k} \mathcal{N}^{\bar{x}^{\prime}}$. By property (1), it is equivariant with respect to the group-indscheme $\prod_{j=1, \ldots, m} N^{-}\left(\left(t_{j}^{\prime}\right)\right)$.

This implies our assertion, since the above group-indscheme acts transitively along the fibers of the composed map

$$
{ }_{\check{\nu}}^{k} \operatorname{Bun}_{N^{-}} \underset{\nu}{k}{\frac{\times}{\operatorname{Bun}_{N^{-}}}}^{k} \mathcal{N}^{\bar{x}^{\prime}} \rightarrow\left(G / N^{-}\right)\left([t] / t^{k}\right) .
$$

Thus, it remains to show that condition (3) on $\mathcal{F}$ implies that the perverse sheaf $\mathcal{F}^{\prime}$ on $\left(G / N^{-}\right)\left([t] / t^{k}\right)$ comes as a pull-back from a perverse sheaf on $\left(G / B^{-}\right)\left([t] / t^{k}\right)$. I.e., we have to show that $\mathcal{F}^{\prime}$ is equivariant with respect to $T\left([t] / t^{k}\right)$. Note that the equivariance with respect to the subgroup $T \subset T\left([t] / t^{k}\right)$ follows from the assumption that $\mathcal{F}$ on ${ }_{\breve{\nu}}^{k} \operatorname{Bun}_{N^{-}}$was $T$-equivariant. Thus, it remains to check the equivarince property with respect to the unipotent subgroup $\operatorname{ker}\left(T\left([t] / t^{k}\right) \rightarrow T\right)$.

For $\check{\mu}$ such that $\check{\mu}+\check{\nu} \in \check{\Lambda}^{\text {pos }}$, consider the composed map

$$
\underset{\nu}{k} \stackrel{\circ}{\mathfrak{F}} \rightarrow\left(G / N^{-}\right)\left([t] / t^{k}\right),
$$

where $\underset{\nu}{k} \stackrel{\circ}{\mathfrak{F}^{\breve{\mu}}}$ is the fiber of $\underset{\nu}{k} \stackrel{\circ}{Z^{\breve{\mu}}}$ over $\check{\mu} \cdot x \in{ }_{\infty} X^{\check{\mu}}$. The above map is equivariant with respect to $T[[t]]$ acting on the two sides. Moreover, it is surjective if $\breve{\mu}$ was chosen large enough.

Let $k^{\prime} \geq k$ be such that the action of $T[[t]]$ on $\underset{\nu}{k} \stackrel{\circ}{\mathfrak{F}^{\breve{\mu}}}$ factors through $T\left([t] / t^{k^{\prime}}\right)$. Let $\bar{m}$ be large enough, so that the map $\operatorname{Maps}(X, T)^{\bar{m}} \rightarrow T\left([t] / t^{k^{\prime}}\right)$, given by Taylor expansion at $x$, is surjective.

Property (3) for this $\bar{m}$ implies then that the restriction of $\mathcal{F}$ to $\underset{\nu}{k} \stackrel{\circ}{\mathfrak{F}} \breve{\mu}$ is $T\left([t] / t^{k^{\prime}}\right)$ equivariant. This implies that $\mathcal{F}^{\prime}$ is also equivariant with respect to this group.

4.2.4. We will now investigate the mutual dependence of conditions (1) and (2). For a natural number $m$ consider the product $\stackrel{\circ}{X}^{m} \times{ }_{\infty}^{k} \overline{\operatorname{Bun}}_{N^{-}}$, and let $\left(\stackrel{\circ}{X}{ }^{m} \times{ }_{\infty}^{k} \overline{\operatorname{Bun}}_{N^{-}}\right)^{n . z \text {. }}$ denote the open subset, corresponding to the condition that the zeros of the maps $\kappa^{\lambda}$ are away from the $m$ marked points of $X^{m}$. In other words, the fiber of this space over a given $\bar{x}^{\prime} \in \stackrel{\circ}{X}^{m}$ is the stack that we denoted by ${ }_{\infty}^{k} \overline{\operatorname{Bun}}_{N^{-}}^{\text {n.z. }} \bar{x}^{\prime}$.

Over $X^{m}$ we have a group-scheme, denoted $\operatorname{Jets}^{+}\left(N^{-}\right)^{m}$, whose fiver over $\bar{x}^{\prime}=$ $\left\{x_{1}^{\prime}, \ldots, x_{m}^{\prime}\right\}$ is $\Pi N^{-}\left[\left[t_{j}^{\prime}\right]\right]$, where the product is taken over distinct points among the $x_{i}^{\prime}$ 's. In addition, we have a group-indscheme, denoted $\operatorname{Jets}\left(N^{-}\right)^{m}$, whose fiber over the same collection of points is $\Pi N^{-}\left(\left(t_{j}^{\prime}\right)\right)$. Since $N^{-}$is unipotent, this group-indscheme can be represented as a union of its closed group-subschemes.

Finally, over $\left(X^{m} \times{ }_{\infty}^{k} \overline{\operatorname{Bun}}_{N^{-}}\right)^{n . z \text { t }}$ there exists a canonical Jets ${ }^{+}\left(N^{-}\right)^{m}$-torsor, which we will denote by ${ }^{k} \mathcal{N}^{m}$. The action of $\operatorname{Jets}^{+}\left(N^{-}\right)^{m}$ on ${ }^{k} \mathcal{N}^{m}$ extends to an action of $\operatorname{Jets}\left(N^{-}\right)^{m}$. 
Lemma 4.2.5. Let $\mathcal{F}$ be a perverse sheaf on ${ }_{\infty}^{k} \overline{\mathrm{Bun}}_{N^{-}}$, which satisfies property (1) of Sect. 4.2.1. Then the pull-back of $\mathcal{F}$ to ${ }^{k} \mathcal{N}^{m}$ is equivariant with respect to $\operatorname{Jets}\left(N^{-}\right)^{m}$.

This follows from the fact "fiber-wise equivariance" implies "equivariance" for a unipotent group-scheme.

Remark. Arguing as in [FGV], Sect. 6.2, one can show that condition (1) is equivalent to the following, seemingly weaker, condition. Namely, it is sufficient to impose the $N^{-}\left(\left(t^{\prime}\right)\right)$-equivariance condition for just one fixed point $x^{\prime}$ distinct from $x$.

Let us say that a perverse sheaf $\mathcal{F}$ on ${ }_{\infty}^{k} \overline{\operatorname{Bun}}_{N^{-}}$has a weak factorization property if, in

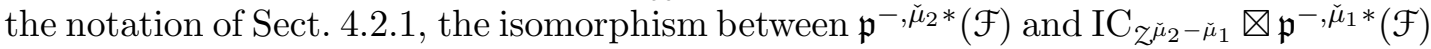
holds over the open subset

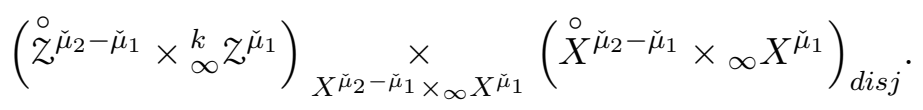

Since $\stackrel{\circ}{Z} \check{\mu}_{2}-\check{\mu}_{1}$ is smooth, this condition is equivalent to the restriction of $\mathfrak{p}^{-, \check{\mu}_{2} *}(\mathcal{F})$ to the above open subset being constant along the first factor.

Proposition 4.2.6. For a perverse sheaf $\mathcal{F}$ on ${ }_{\infty}^{k} \overline{\operatorname{Bun}}_{N^{-}}$, property (1) is equivalent to the weak factorization property.

Before giving a proof let us make the following observation: we have two maps

$$
\overleftarrow{h}_{N^{-}}, \vec{h}_{N^{-}}: \operatorname{Jets}\left(N^{-}\right)^{m} \stackrel{\operatorname{Jets}^{+}\left(N^{-}\right)^{m}}{\times}{ }^{\mathcal{N}^{m}} \rightarrow{ }_{\infty}^{k} \overline{\operatorname{Bun}}_{N^{-}}
$$

the first being the tautological projection, and the second is given by the action of $\operatorname{Jets}\left(N^{-}\right)^{m}$ on ${ }^{k} \mathcal{N}^{m}$. If $\check{\mu}_{1}, \check{\mu}_{2} \in \check{\Lambda}$ are two elements, with $\check{\mu}_{2}-\check{\mu}_{1} \in \check{\Lambda}^{\text {pos }}$ such that $m=l\left(\check{\mu}_{2}-\check{\mu}_{1}\right)$ there is a natural projection $\stackrel{\circ}{X^{m}} \rightarrow \stackrel{\circ}{X^{\mu_{2}}-\check{\mu}_{1}}$ and a map

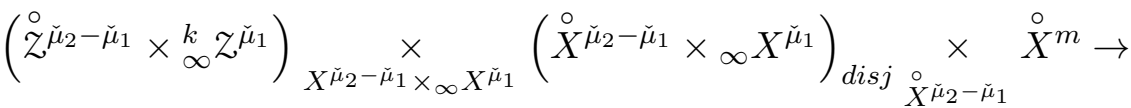

$$
\begin{aligned}
& \operatorname{Jets}(N)^{m} \stackrel{\operatorname{Jets}^{+}(N)^{m}}{\times}{ }^{k} \mathcal{N}^{m},
\end{aligned}
$$

such that its composition with $\overleftarrow{h}_{N}$ is the projection

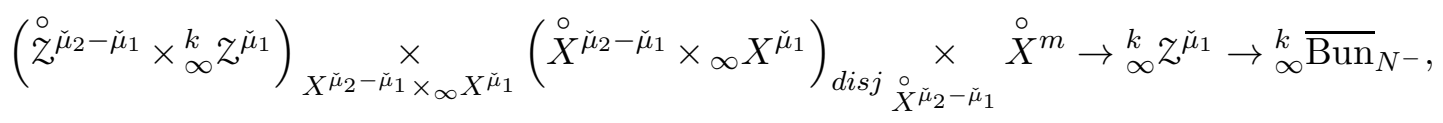

and its composition with $\vec{h}_{N}$ identifies via Lemma 4.1 .5 with

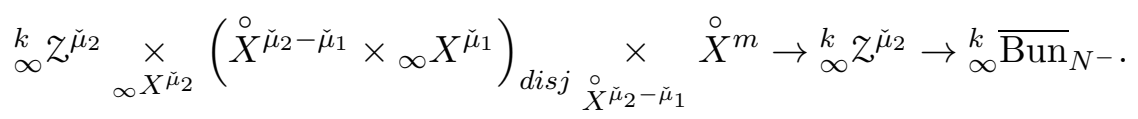

Now let us proof the proposition:

Proof. Assume first that $\mathcal{F}$ satisfies property (1), and hence, by Proposition 4.2.5, its pull-back to ${ }^{k} \mathcal{N}^{m}$ is $\operatorname{Jets}\left(N^{-}\right)^{m}$-equivariant. We obtain that the restrictions of $\overleftarrow{h}_{N^{-}}^{*}(\mathcal{F})$ and $\vec{h}_{N^{-}}^{*}(\mathcal{F})$ to any finite-dimensional subscheme of $\operatorname{Jets}\left(N^{-}\right)^{m} \stackrel{\operatorname{Jets}^{+}\left(N^{-}\right)^{m}}{\times} \mathcal{N}^{m}$ are 
isomorphic. Then the weak factorizability of $\mathcal{F}$ follows from the properties of the map from (33) above.

To prove the implication in the opposite direction, we reverse the steps. We have to show that for a given finite collection of distinct points $\bar{x}^{\prime}=\left\{x_{1}^{\prime}, \ldots, x_{m}^{\prime}\right\}$, the restrictions of $\overleftarrow{h}_{N^{-}}$and $\vec{h}_{N^{-}}$to the fiber of $\operatorname{Jets}\left(N^{-}\right)^{m} \stackrel{\operatorname{Jets}^{+}\left(N^{-}\right)^{m}}{\times}{ }^{k} \mathcal{N}^{m}$ over $\bar{x}^{\prime} \in X^{m}$ are isomorphic over every finite-dimensional subscheme of this ind-scheme. Since each $N^{-}\left(\left(t_{j}^{\prime}\right)\right)$ is a union of pro-unipotent subgroups, it is sufficient to show that the isomorphism holds after the base change with respect to

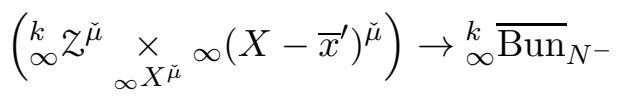

for $\check{\mu}$ large enough.

Note that the above fiber, base-changed to ${ }_{\infty}^{k} z^{\check{\mu}}$, is isomorphic to

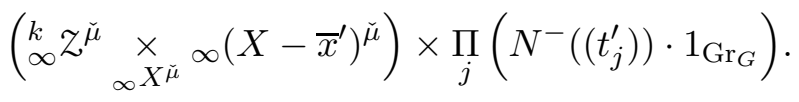

Our assertion follows now from $(28)$, since $N^{-}\left(\left(t_{j}^{\prime}\right)\right) \cdot 1_{\mathrm{Gr}_{G}}$ can be exhausted by affine subspaces, each of which contains as a dense subset the intersection

$$
\left(N\left(\left(t_{j}^{\prime}\right)\right) \cdot \check{\mu}^{\prime}\right) \cap\left(N^{-}\left(\left(t_{j}^{\prime}\right)\right) \cdot 1_{\mathrm{Gr}_{G}}\right)
$$

for some $\check{\mu}^{\prime}$.

As a corollary of the first assertion of the proposition, we obtain the following:

Corollary 4.2.7. Let $0 \rightarrow \mathcal{F}_{1} \rightarrow \mathcal{F} \rightarrow \mathcal{F}_{2} \rightarrow 0$ be a short exact sequence of objects of $\operatorname{Perv}\left({ }_{\infty}^{k} \overline{\operatorname{Bun}}_{N^{-}}\right)^{T}$, with $\mathcal{F}_{1}, \mathcal{F}_{2}$ satisfying properties (1) and (2) of the definition of $\operatorname{Perv}\left(\mathcal{F} l^{\frac{\infty}{2}}\right)^{k}$. Then $\mathcal{F}$ also satisfies properties (1) and (2).

Proof. Since the group $N^{-}\left(\left(t^{\prime}\right)\right)$ is (ind)-pro-unipotent, the only non-trivial condition to check is the factorizability property. For $\mathcal{F}$ as above, its pull-back to

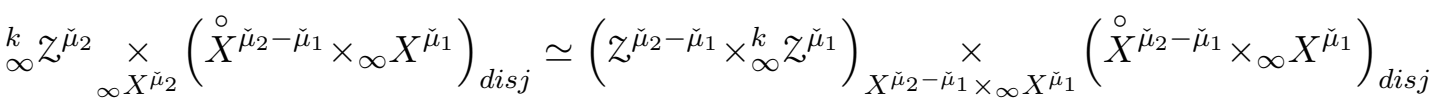

is the Goresky-MacPherson extension from the open sub-space

$$
\left(\stackrel{\circ}{Z}^{\check{\mu}_{2}-\check{\mu}_{1}} \times{ }_{\infty}^{k} Z^{\check{\mu}_{1}}\right)_{X^{\check{\mu}_{2}-\check{\mu}_{1}} \times \infty X^{\check{\mu}_{1}}}\left(\stackrel{\circ}{X^{\check{\mu}_{2}-\check{\mu}_{1}}} \times_{\infty} X^{\check{\mu}_{1}}\right)_{d i s j} .
$$

However, the latter is constant along the $\stackrel{\stackrel{\circ}{z}^{\breve{\mu}}}{\breve{\mu}_{2}} \breve{\mu}_{1}$-factor because of property (1), Lemma 4.2.5 and Proposition 4.2.6. Along the ${ }_{\infty}^{k} z^{\check{\mu}_{1}}$ factor it is isomorphic to $\mathfrak{p}^{-, \breve{\mu}_{1} *}(\mathcal{F})$ by (33). 
4.2.8. Our present goal is to establish the following:

Proposition 4.2.9. The category $\operatorname{Perv}\left(\mathcal{F} l^{\frac{\infty}{2}}\right)^{G^{k}}$, as a subcategory of the category of T-equivariant perverse sheaves on ${ }_{\infty}^{k} \overline{\mathrm{Bun}}_{N^{-}}$, is stable under extensions.

The rest of the present subsection is devoted to this proposition. In view of Corollary 4.2.7, we have to show that if $0 \rightarrow \mathcal{F}_{1} \rightarrow \mathcal{F} \rightarrow \mathcal{F}_{2} \rightarrow 0$ is a short exact sequence in $\operatorname{Perv}\left({ }_{\infty}^{k} \overline{\operatorname{Bun}}_{N^{-}}\right)^{T}$ with $\mathcal{F}_{1}, \mathcal{F}_{2} \in \operatorname{Perv}\left(\mathcal{F l} l^{\frac{\infty}{2}}\right)^{G^{k}}$, then $\mathcal{F}$ satisfies property (3).

Consider the pull-back

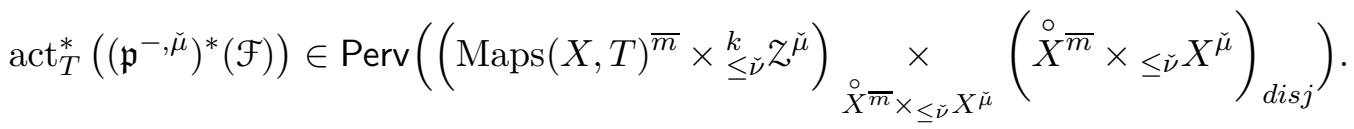

Since $\operatorname{Maps}(X, T)^{\bar{m}}$ is isomorphic to the affine space, it is sufficient to show that the restriction of the above pull-back to the fiber over every geometric point $\mathbf{z}:=$ $\left(\mathcal{P}_{G},\left\{\kappa^{\lambda}\right\},\left\{\kappa^{\lambda,-}\right\}\right) \in_{\infty}^{k} z^{\check{\mu}}$ is a complex with constant cohomologies.

By the factorization property, it is sufficient to consider the case when the point ${ }_{\infty}^{k} \mathfrak{s}^{\check{\mu}}(\mathbf{z}) \in{ }_{\infty} X^{\check{\mu}}$ equals $\check{\mu} \cdot x$. In this case, the map act $T_{T}^{*}$ factors through the action of $\operatorname{ker}(T[[t]] \rightarrow T)$ on ${ }_{\infty}^{k} \mathfrak{F}^{\check{\mu}}$.

Hence, it is sufficient to check that the restriction of $\mathcal{F}$ to ${ }_{\infty}^{k} \mathfrak{F}^{\check{\mu}}$ is $\operatorname{ker}(T[[t]] \rightarrow T)$ equivariant. But the above restriction is an extension of the restrictions of $\mathcal{F}_{1}$ and $\mathcal{F}_{2}$. Since for $\bar{m}^{\prime}$ large enough the map $\operatorname{Maps}(X, T)^{\bar{m}^{\prime}} \rightarrow \operatorname{ker}(T[[t]] \rightarrow T)$ is surjective with connected fibers, the fact that $\mathcal{F}_{1}$ and $\mathcal{F}_{2}$ satisfy property $(3)$ implies that their restrictions to ${ }_{\infty}^{k} \mathfrak{F}^{\check{\mu}}$ are $\operatorname{ker}(T[[t]] \rightarrow T)$-equivariant. This proves our assertion, since $\operatorname{ker}(T[[t]] \rightarrow T)$ is pro-unipotent, and hence the equivariance property is stable under extensions.

Thus, Proposition 4.2.9 is proved. As a by-product we obtain the following alternative way to spell out condition (3):

Corollary 4.2.10. Let $\mathcal{F} \in \operatorname{Perv}\left({ }_{\infty}^{k} \overline{\operatorname{Bun}}_{N^{-}}\right)^{T}$ be a perverse sheaf, satisfying properties (1) and (2) from the definition of $\operatorname{Perv}\left(\mathcal{F} l^{\frac{\infty}{2}}\right)^{G^{k}}$. The the following are equivalent:

(1) $\mathcal{F}$ satisifies also property (3).

(2) The *- (or !-) restrictions of $\mathcal{F}$ to every ${ }_{\check{\nu}}^{k} \operatorname{Bun}_{N^{-}}$are such that their perverse cohomologies are pull-backs from $T\left([t] / t^{k}\right)$-equivariant perverse sheaves on $G / N^{-}\left([t] / t^{k}\right)$.

(3) The *- (or !-) restrictions of $\left(\mathfrak{p}^{\check{\mu},-}\right)^{*}(\mathcal{F})$ to every ${ }_{\infty}^{k} \mathfrak{F}^{\check{\mu}}$ is $T[[t]]$-equivariant.

4.2.11. Recall that for $\check{\nu} \in \check{\Lambda}$, we have introduced the category

$$
{ }_{\check{\nu}}^{\prime} \operatorname{Perv}\left(\mathcal{F} l^{\frac{\infty}{2}}\right)^{G^{k}} \subset \operatorname{Perv}\left({ }_{\check{\nu}}^{k} \overline{\operatorname{Bun}}_{N^{-}}\right),
$$

which is equivalent to

$$
{ }_{\check{\nu}} \operatorname{Perv}\left(\mathcal{F} l^{\frac{\infty}{2}}\right)^{G^{k}} \subset \operatorname{Perv}\left({ }_{\check{\nu}}^{k} \operatorname{Bun}_{N}\right) .
$$


MODULES OVER THE SMALL QUANTUM GROUP AND SEMI-INFINITE FLAG MANIFOLD 57

Proposition 4.2.12. Let $\mathcal{F}^{\prime} \in \in_{\check{\nu}}^{\prime} \operatorname{Perv}\left(\mathcal{F} l^{\frac{\infty}{2}}\right)^{G^{k}}$ be such that $\left(\bar{i}_{\breve{\nu}}\right) !\left(\mathcal{F}^{\prime}\right)$ is a perverse sheaf. Then for $\mathcal{F} \in \operatorname{Perv}\left(\mathcal{F} l^{\frac{\infty}{2}}\right)^{G^{k}}$ the canonical map

$$
\operatorname{Ext}_{\infty}^{1} \overline{\operatorname{Bun}}_{N^{-}}\left(\left(\bar{i}_{\check{\nu}}\right) !\left(\mathcal{F}^{\prime}\right), \mathcal{F}\right) \rightarrow \operatorname{Ext}_{k_{\infty}}^{1} \overline{\operatorname{Bun}}_{N^{-}}\left(\left(i_{\check{\nu}}\right) !\left(\mathcal{F}^{\prime}\right), \mathcal{F}\right)
$$

is an isomorphism.

Note that due to Proposition 4.2.9, the above proposition can be reformulated as follows:

$$
\operatorname{Ext}_{\left.\operatorname{Perv}(\mathcal{F})^{\frac{\infty}{2}}\right)^{k}}\left(\left(\bar{i}_{\check{\nu}}\right)_{!}\left(\mathcal{F}^{\prime}\right), \mathcal{F}\right) \simeq \mathrm{R}^{1} \operatorname{Hom}_{\check{\nu}} \operatorname{Bun}_{N}\left(\mathcal{F}^{\prime}, i_{\check{\nu}}^{!}(\mathcal{F})\right) .
$$

Proof. The fact that the map in question is injective is evident, since $\left(i_{\check{\nu}}\right)_{!}\left(\mathcal{F}^{\prime}\right)$ surjects onto $\left(\bar{i}_{\breve{\nu}}\right) !\left(\mathcal{F}^{\prime}\right)$, and $\mathcal{F}$ has no sub-objects supported on ${ }_{\check{\nu}}^{k} \overline{\operatorname{Bun}}_{N^{-}}-{ }_{\check{\nu}}^{k} \operatorname{Bun}_{N^{-}}$.

To prove the surjectivity we can replace ${ }_{\infty}^{k} \overline{\operatorname{Bun}}_{N^{-}}$by its open sub-stack ${ }_{\geq \check{\nu}}^{k} \overline{\operatorname{Bun}}_{N^{-}}$, which is obtained by removing from ${ }_{\infty}^{k} \overline{\mathrm{Bun}}_{N^{-}}$all ${\underset{\leq \breve{\nu}}{\prime}}_{\check{\mathrm{Bun}}_{N^{-}}}$for $\check{\nu}^{\prime}<\check{\nu}$. Evidently,

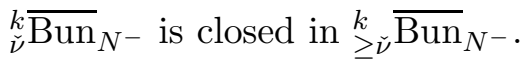

Let

$$
0 \rightarrow \mathcal{F} \rightarrow \mathcal{F}_{1} \rightarrow\left(i_{\check{\nu}}\right) !\left(\mathcal{F}^{\prime}\right) \rightarrow 0
$$

be an extension. We have to show that it is induced from an extension of $\left(\bar{i}_{\check{\nu}}\right)_{!}\left(\mathcal{F}^{\prime}\right)$ by $\mathcal{F}$. Let $\tilde{\mathcal{F}}_{1}$ be the perverse sheaf on ${ }_{\geq \nu}^{k} \overline{\mathrm{Bun}}_{N^{-}}$obtained as a Goresky-MacPherson of the restriction of $\mathcal{F}_{1}$ to the open substack

$$
\stackrel{\nu}{\geq}_{\check{\nu}}^{k} \overline{\operatorname{Bun}}_{N^{-}}-\left({ }_{\nu}^{k} \overline{\operatorname{Bun}}_{N^{-}}-{ }_{\check{\nu}}^{k} \operatorname{Bun}_{N^{-}}\right) .
$$

We claim that $\tilde{\mathcal{F}}_{1}$ is the desired extension. Namely, we have the maps

$$
\mathcal{F} \hookrightarrow \tilde{\mathcal{F}}_{1} \rightarrow\left(\bar{i}_{\check{\nu}}\right) !\left(\mathcal{F}^{\prime}\right),
$$

and we claim that this is a short exact sequence.

To check this, by Proposition 4.2.3(3), it is enough to show that $\tilde{\mathcal{F}}_{1}$ is an object of the corresponding category $\geq \check{\nu} \operatorname{Perv}\left(\mathcal{F} l^{\frac{\infty}{2}}\right)^{G^{k}}$. However, properties (1) and (3) are automatic, and the factorization property (2) follows by combining Proposition 4.2.6 and the definition of Goresky-MacPherson extension.

The 5-lemma yields:

Corollary 4.2.13. For $\mathcal{F}$ as in the proposition, the natural map

$$
\operatorname{Ext}_{\operatorname{Perv}\left(\mathcal{F l}^{2} \frac{\infty}{2}\right) G^{k}}^{2}\left(\left(\bar{i}_{\check{\nu}}\right) !\left(\mathcal{F}^{\prime}\right), \mathcal{F}\right) \rightarrow \mathrm{R}^{2} \operatorname{Hom}_{\check{\nu}} \operatorname{Bun}_{N}\left(\mathcal{F}^{\prime}, i_{\check{\nu}}^{!}(\mathcal{F})\right)
$$

is injective.

Remark. ¿From Proposition 4.2.12 one can formally deduce that the maps

$$
\operatorname{Ext}_{\operatorname{Perv}\left(\mathcal{F l}^{i} \frac{\infty}{2}\right) G^{k}}^{i}\left(\left(\bar{i}_{\check{\nu}}\right)_{!}\left(\mathcal{F}^{\prime}\right), \mathcal{F}\right) \rightarrow \mathrm{R}^{i} \operatorname{Hom}_{\check{\nu}} \operatorname{Bun}_{N}\left(\mathcal{F}^{\prime}, i_{\check{\nu}}^{!}(\mathcal{F})\right)
$$

are isomorphisms for all $i$.

\subsection{The spherical case.}


4.3.1. Let $\operatorname{Perv}\left(\mathcal{F} l^{\frac{\infty}{2}}\right)^{G[t]]]} \operatorname{denote} \operatorname{Perv}\left(\mathcal{F} l^{\frac{\infty}{2}}\right)^{G^{k}}$ for $k=0$; this is a full subcategory in ${ }_{\infty} \overline{\operatorname{Bun}}_{N^{-}}$. For $\check{\nu} \in \check{\Lambda}$ we will denote by $\mathrm{IC}_{\check{\nu}}$ the corresponding irreducible, i.e.,

$$
\mathrm{IC}_{\check{\nu}} \simeq\left(i_{\check{\nu}}\right)_{! *}\left(\mathrm{IC}_{\check{\nu} \operatorname{Bun}_{N}}\right) \simeq\left(\bar{i}_{\check{\nu}}\right)_{! *}\left(\mathrm{IC}_{\check{\nu}} \overline{\operatorname{Bun}}_{N^{-}}\right) .
$$

These are the irreducible objects of $\operatorname{Perv}\left(\mathcal{F} l^{\frac{\infty}{2}}\right)^{G[[t]]}$.

Proposition 4.3.2. The category $\operatorname{Perv}\left(\mathcal{F} l^{\frac{\infty}{2}}\right)^{G[[t]]}$ is semi-simple.

Proof. It would be enough to show that if $\mathrm{IC}_{\check{\nu}_{1}}$ and $\mathrm{IC}_{\check{\nu}_{2}}$ are two simple objects of $\operatorname{Perv}\left(\mathcal{F} l^{\frac{\infty}{2}}\right)^{G[t]]}$, whose support is contained in some $\leq \overline{\mathrm{B}}_{\mathrm{Bun}_{N^{-}}}$, then over some open substack of $\leq \check{\nu} \overline{\mathrm{Bun}}_{N^{-}}, \operatorname{Ext}^{1}\left(\mathrm{IC}_{\check{\nu}_{1}}, \mathrm{IC}_{\check{\nu}_{2}}\right)$ is zero.

Let $\check{\nu}_{1}, \check{\nu}_{2}$ be two elements of $\check{\Lambda}$. In order for $\operatorname{Ext}^{1}\left(\mathrm{IC}_{\check{\nu}_{1}}, \mathrm{IC}_{\check{\nu}_{2}}\right)$ to be non-trivial, the support of one sheaf must be contained in the closure of the support of the other. This means that either $\check{\nu}_{1} \leq \check{\nu}_{2}$ or $\check{\nu}_{2} \leq \check{\nu}_{1}$. By Verdier duality we can assume that $\check{\nu}_{1} \leq \check{\nu}_{2}$.

Consider the open sub-stack of $\leq \check{\nu}_{2} \overline{\mathrm{Bun}}_{N^{-}}$obtained by removing the closed sub-stack $\leq \check{\nu}_{1} \overline{\operatorname{Bun}}_{N^{-}}-\check{\nu}_{1} \operatorname{Bun}_{N}$. As in [FGV], Sect. 6.1.4,

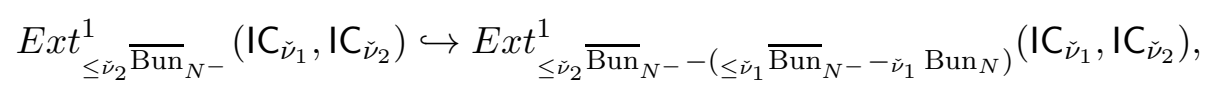

so it is enough to show that the latter is 0 . Since

$$
\check{\nu}_{1} \operatorname{Bun}_{N} \subset \leq \check{\nu}_{2} \overline{\operatorname{Bun}}_{N^{-}}-\left(\leq \check{\nu}_{1} \overline{\operatorname{Bun}}_{N^{-}}-\check{\nu}_{1} \operatorname{Bun}_{N}\right)
$$

is closed, the latter $\mathrm{Ext}^{1}$ is isomorphic to

$$
\mathrm{R}^{1} \operatorname{Hom}_{\check{\nu}_{1}} \operatorname{Bun}_{N}\left(\mathrm{IC}_{\check{\nu}_{1}} \operatorname{Bun}_{N}, i_{\check{\nu}_{1}}^{!}\left(\mathrm{IC}_{\check{\nu}_{2}} \operatorname{Bun}_{N}\right)\right)
$$

There are two cases: if $\check{\nu}_{1}<\check{\nu}_{2}$, then we are done by $[\mathrm{BFGM}]$, since $i_{\check{\nu}_{1}}^{!}\left(\mathrm{IC}_{\check{\nu}_{2}} \operatorname{Bun}_{N}\right)$ lives in the cohomological degrees $\geq 2$.

If $\check{\nu}=\check{\nu}_{1}=\check{\nu}_{2}$, then the assertion follows from the fact that $\check{\nu}_{\nu} \operatorname{Bun}_{N}$ is $\operatorname{simply-}^{-}$ connected, cf. [FGV], Sect. 6 .

4.3.3. Consider the object of $\mathrm{D}\left({ }_{\infty} \overline{\mathrm{Bun}}_{N^{-}}\right)$equal to $\left(\bar{i}_{\check{\nu}}\right)_{!}\left(\mathrm{IC}_{\check{\nu}} \overline{\mathrm{Bun}}_{N^{-}}\right)$. This is a complex that lives in non-positive cohomological degrees, and each of its perverse cohomologies is an object of $\operatorname{Perv}\left(\mathcal{F} l^{\frac{\infty}{2}}\right)^{G[[t]]}$, by Proposition 4.2.3.

Theorem 4.3.4. The $-k$-th perverse cohomology of $\left(\bar{i}_{\check{\nu}}\right)_{!}\left(\mathrm{IC}_{\check{\nu}} \overline{\mathrm{Bun}}_{N^{-}}\right)$is isomorphic to the direct sum over collections of $k$ distinct positive roots $\left\{\beta_{1}, \ldots, \beta_{k}\right\}$ of

$$
\mathrm{IC}_{\check{\nu}-\sum_{j} \beta_{j}} .
$$

Corollary 4.3.5. The complex $\left(\bar{i}_{\breve{\nu}}\right)_{!}\left(\mathrm{IC}_{\check{\nu}} \overline{\operatorname{Bun}}_{N^{-}}\right)$(resp., $\left(\bar{i}_{\breve{\nu}}\right)_{*}\left(\mathrm{IC}_{\check{\nu}} \overline{\operatorname{Bun}}_{N^{-}}\right)$) lives in the cohomological degrees $[-\operatorname{dim}(\mathfrak{n}), 0]($ resp., $[0, \operatorname{dim}(\mathfrak{n})])$ and its $-\operatorname{dim}(\mathfrak{n})-(\operatorname{dim}(\mathfrak{n})-) d e-$ gree cohomology is isomorphic to $\mathrm{IC}_{\check{\nu}-2 \check{\rho}}$. 
The rest of this subsection is devoted to the proof of the above theorem. For $\check{\mu} \in \check{\Lambda}^{\text {pos }}$ consider the stack $\leq \check{\nu} \overline{\mathrm{Bun}}_{N^{-}}$, fibered over $X^{\check{\mu}}$, classifying pairs $\left(D \in X^{\check{\mu}},\left\{\kappa^{\lambda}\right\}\right)$ such that each $\kappa^{\lambda}$ factors as

$$
V_{\mathcal{P}_{G}}^{\lambda} \rightarrow \mathcal{O}_{X}(\lambda(\check{\nu} \cdot x-D)) \rightarrow \mathcal{O}_{X}
$$

Let $\check{\nu}_{\bar{\nu}} \operatorname{Bun}_{N^{-}}^{\check{\mu}}$ be the open sub-stack of ${ }_{\leq \check{\nu}} \overline{\operatorname{Bun}}_{N^{-}}^{\leq \breve{\mu}}$, corresponding to the condition that the maps $V_{\mathcal{P}_{G}}^{\lambda} \rightarrow \mathcal{O}_{X}(\lambda(\check{\nu} \cdot x-D))$ above, are bundle maps.

It is easy to see that $\check{\nu} \operatorname{Bun}_{N^{-}}^{\check{\mu}}$ is smooth over $X^{\check{\mu}}$. The projection $\leq \check{\nu} \overline{\operatorname{Bun}}_{N^{-}} \leq \check{\mu} \rightarrow X^{\check{\mu}}$ is ULA (universally locally acyclic) with respect to the IC sheaf on this stack, by [BG], Sect. 5.2.

We let $\bar{i}^{\leq \check{\mu}}$ (resp., $i^{\check{\mu}}$ ) denote the natural maps from the above stacks to $\leq \check{\nu} \overline{\operatorname{Bun}}_{N^{-}}$. By $[\mathrm{BG}], \bar{i}^{\leq \check{\mu}}$ is finite (and, in particular, proper), and $i^{\check{\mu}}$ is a locally closed embedding. Moreover, by $[\mathrm{FGV}]$, Sect. 3.3, $i^{\breve{\mu}}$ is affine. In particular, every $i_{!}^{\breve{\mu}}\left(\mathrm{IC}_{\check{\nu}} \operatorname{Bun}_{N^{-}}^{\breve{\mu}}\right)$ is a perverse sheaf.

The following is a reformulation of the main result of [FFKM] and [BFGM]:

Theorem 4.3.6. The $k$-th cohomology of $\left(i^{\check{\mu}}\right)^{*}\left(\mathrm{IC}_{\check{\nu}}\right)$ is isomorphic to the direct sum over the set of partitions $\mathfrak{P}$

$$
\check{\mu}=\Sigma m_{j} \cdot \beta^{j}, \beta^{j} \neq \beta^{j^{\prime}}, \Sigma m_{j}=k,
$$

where $\beta^{i}$ 's are positive roots, of the direct images of the shifted by $[k]$ constant perverse sheaves on each

$$
X^{\mathfrak{P}} \underset{X^{\check{\mu}}}{\times} \check{\operatorname{Linn}} \underset{N^{-}}{\check{\mu}},
$$

where $X^{\mathfrak{P}} \simeq \prod_{j} X^{\left(m_{j}\right)}$, that maps naturally to $X^{\check{\mu}}$.

For each partition $\mathfrak{P}$ as above let $\mathcal{E}_{\mathfrak{P}}$ be the perverse sheaf on $X^{\check{\mu}}$, equal to the direct image under $X^{\mathfrak{P}} \rightarrow X^{\check{\mu}}$ of the irreducible perverse sheaf obtained by taking the external product over $j$ of the 1-dimensional local systems on each $X^{\left(m_{j}\right)}$ - Diag with monodromy -1 around the diagonal. By the ULA property of $\leq \check{\nu} \overline{\operatorname{Bun}}_{N^{-}} \leq \check{\text { over }} X^{\check{\mu}}$, the

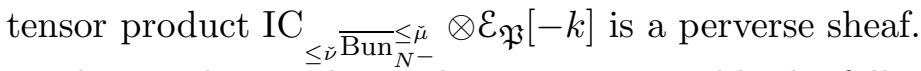

The usual Koszul complex argument yields the following:

Corollary 4.3.7. Irreducible constituents of $\left(i_{\nu}\right) !\left(\mathrm{IC}_{\check{\nu} \mathrm{Bun}_{N}}\right)$ are the perverse sheaves $\bar{i}_{*}^{\leq \check{\mu}}\left(\mathrm{IC}_{\leq \check{\nu}} \overline{\operatorname{Bun}}_{N^{-}}^{\leq \check{\mu}} \otimes \mathcal{E}_{\mathfrak{P}}[-k]\right)$ for all $\check{\mu} \in \check{\Lambda}$ and partitions $\mathfrak{P}$, each appearing once.

Recall that $\leq \check{\nu} \mathfrak{F}^{\check{\mu}}$ denotes the fiber of $\leq \check{\nu} \check{\chi}^{\check{\mu}}$ over $\check{\mu} \cdot x \in{ }_{\infty} X^{\check{\mu}}$. By [BFGM], we have:

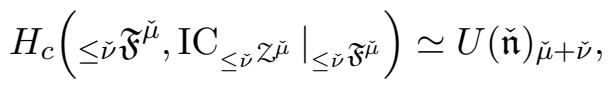

in particular, the above cohomology is concentrated in cohomological degree 0 .

Combining this result with Corollary 4.3.7, and taking into account that the restriction of $\mathcal{E}_{\mathfrak{P}}$ to the diagonal divisor is 0 unless all $m_{j}=1$, we obtain the following: 
Corollary 4.3.8. The cohomology group

$$
H_{c}^{-k}\left(\leq \check{\nu} \mathfrak{F}^{\check{\mu}},\left.\left(i_{\nu}\right) !\left(\mathrm{IC}_{\check{\nu}}^{\circ} \check{Z}_{\breve{\mu}}\right)\right|_{\left.\leq \check{\nu} \mathfrak{F}^{\check{\mu}}\right)}\right.
$$

is the direct sum over $\check{\lambda} \in \check{\Lambda}^{\text {pos }}$ of $U(\check{\mathfrak{n}})_{\check{\mu}+\check{\nu}-\check{\lambda}}$, each appearing the number of times equal to the number of partitions of $\check{\lambda}$ as a sum of $k$ distinct positive roots.

Let us note that the intersection $\stackrel{\stackrel{\circ}{\mathfrak{F}}}{\breve{\mu}}:=\infty \mathfrak{F}^{\check{\mu}} \cap_{\check{\nu}} \stackrel{\circ}{z^{\breve{\mu}}}$ is isomorphic to

$$
(N((t)) \cdot(\check{\nu}+\check{\mu})) \cap\left(N^{-}((t)) \cdot 1_{\mathrm{Gr}_{G}}\right) .
$$

Thus, Corollary 4.3.8 gives an expression for

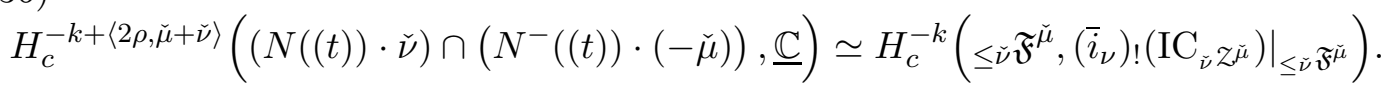

Now we can finish the proof of Theorem 4.3.4, essentially be reversing the logic. We have to show that the multiplicity $m_{k}(\check{\lambda})$ of $\mathrm{IC}_{\check{\nu}-\check{\lambda}}$ in the $-k$-th perverse cohomology of $\left(\bar{i}_{\check{\nu}}\right)_{!}\left(\mathrm{IC}_{\check{\nu}} \overline{\operatorname{Bun}}_{N^{-}}\right)$equals the number of partitions of $\check{\lambda}$ as a sum of $k$ distinct positive roots, i.e., $\operatorname{dim}\left(\Lambda^{k}(\mathfrak{n})_{\check{\lambda}}\right)$.

We will argue by induction on $\check{\lambda}$, so we can assume that the assertion is known for all $\check{\lambda}^{\prime}<\check{\lambda}$. Consider the cohomology in (36) for $\check{\mu}=\check{\lambda}-\check{\nu}$. By (35), the contributions of different constituents do not cancel out, and we obtain an equality:

$$
\sum_{\check{\lambda}^{\prime} \in \Lambda^{\text {pos }}} \operatorname{dim}\left(\Lambda^{k}(\mathfrak{n})_{\check{\lambda}^{\prime}}\right) \cdot \operatorname{dim}\left(U(\mathfrak{n})_{\check{\lambda}-\check{\lambda}^{\prime}}\right)=\sum_{\check{\lambda}^{\prime}<\lambda} \operatorname{dim}\left(\Lambda^{k}(\mathfrak{n})_{\check{\lambda}^{\prime}}\right) \cdot \operatorname{dim}\left(U(\mathfrak{n})_{\check{\lambda}-\check{\lambda}^{\prime}}\right)+m_{k}(\lambda) .
$$

This implies the desired equality.

\subsection{The Iwahori case.}

4.4.1. Note that the stack ${ }_{\infty}^{k} \overline{\operatorname{Bun}}_{N^{-}}$is acted on by the group $G\left([t] / t^{k}\right)$. In particular, we have the convolution functors:

$$
\mathrm{D}\left(G\left([t] / t^{k}\right)\right) \times \mathrm{D}\left({ }_{\infty}^{k} \overline{\mathrm{Bun}}_{N^{-}}\right) \rightarrow \mathrm{D}\left({ }_{\infty}^{k} \overline{\mathrm{Bun}}_{N^{-}}\right): \mathcal{S}, \mathcal{F} \mapsto \mathcal{S} \star * \mathcal{F} \text { and } \mathcal{S}, \mathcal{F} \mapsto \mathcal{S} \star \dot{F} .
$$

Moreover, these functors are defined on each of the subcategories

$$
\mathrm{D}\left({ }_{\leq \check{\nu}}^{k} \overline{\mathrm{Bun}}_{N^{-}}\right), \mathrm{D}\left({ }_{\check{\nu}}^{k} \overline{\mathrm{Bun}}_{N^{-}}\right) \text {and } \mathrm{D}\left({ }_{\check{\nu}}^{k} \mathrm{Bun}_{N^{-}}\right) \text {, }
$$

so that the *-convolution commutes in the natural sense with the functors

$$
\left(\bar{i}_{\leq \check{\nu}}\right)_{*},\left(\bar{i}_{\check{\nu}}\right)_{*},\left(i_{\check{\nu}}\right)_{*},\left(\bar{i}_{\leq \check{\nu}}\right)^{!},\left(\bar{i}_{\check{\nu}}\right)^{!},\left(i_{\check{\nu}}\right)^{!},\left(\mathrm{ev}_{\check{\nu}}\right)^{!}
$$

and the !-convolution commutes with the functors

$$
\left(\bar{i}_{\leq \check{\nu}}\right)_{!}=\left(\bar{i}_{\leq \check{\nu}}\right)_{*},\left(\bar{i}_{\check{\nu}}\right) !,\left(i_{\check{\nu}}\right) !,\left(\bar{i}_{\leq \check{\nu}}\right)^{*},\left(\bar{i}_{\check{\nu}}\right)^{*},\left(i_{\check{\nu}}\right)^{*},\left(\operatorname{ev}_{\check{\nu}}\right)^{*}
$$

Lemma 4.4.2. For $\mathcal{F} \in \operatorname{Perv}\left(\mathcal{F} l^{\frac{\infty}{2}}\right)^{G^{k}}$ and any $\mathcal{S} \in \mathrm{D}\left(G\left([t] / t^{k}\right)\right)$, the perverse cohomologies of both $\mathcal{S}^{*} \mathcal{F}$ and $\mathcal{S} \stackrel{!}{\star} \mathcal{F}$ belong to $\operatorname{Perv}\left(\mathcal{F} l^{\frac{\infty}{2}}\right)^{G^{k}}$.

Proof. This follows immediately, since the action of $G\left([t] / t^{k}\right)$ extends to ${ }_{\infty}^{k} z^{\check{\mu}}$, respects the factorization isomorphisms, and commutes with the action of the group-schemes involved in the definition of $\operatorname{Perv}\left(\mathcal{F} l^{\frac{\infty}{2}}\right)^{G^{k}}$. 
In what follows we will be interested in the case $k=1$.

4.4.3. Let us denote by ${ }_{\infty}^{I} \overline{\operatorname{Bun}}_{N^{-}}$(resp., ${ }_{\infty}^{I} \overline{\mathrm{Bun}}_{N^{-}}$) the quotient stack of ${ }_{\infty}^{1} \overline{\operatorname{Bun}}_{N^{-}}$by $B \subset G$ (resp., $N \subset G$ ).

We will denote by

$$
\operatorname{Perv}\left(\mathcal{F l} l^{\frac{\infty}{2}}\right)^{I} \subset \operatorname{Perv}\left({ }_{\infty}^{I} \overline{\operatorname{Bun}}_{N^{-}}\right) \text {and } \operatorname{Perv}\left(\mathcal{F l} l^{\frac{\infty}{2}}\right)^{I^{0}} \subset \operatorname{Perv}\left({ }_{\infty}^{I^{0}} \overline{\operatorname{Bun}}_{N^{-}}\right)
$$

the full subcategories of, consisting of objects, whose pull-back to ${ }_{\infty}^{1} \overline{\mathrm{Bun}}_{N^{-}}$belongs to $\operatorname{Perv}\left(\mathcal{F} l^{\frac{\infty}{2}}\right)^{G^{k}}, k=1$.

For $\check{\nu} \in \check{\Lambda}$, let us denote by ${ }_{\check{\nu}}^{I} \overline{\mathrm{Bun}}_{N^{-}}$(resp., $I_{\check{\nu}}^{0} \overline{\mathrm{Bun}}_{N^{-}}$) the corresponding locally closed substack of ${ }_{\infty}^{I} \overline{\mathrm{Bun}}_{N^{-}}\left(\right.$resp., $\left.{ }_{\infty}^{0} \overline{\mathrm{Bun}}_{N^{-}}\right)$, and by $\overline{\mathrm{ev}}_{\check{\nu}}$ the map from it to $B \backslash G / N^{-}$ (resp., $N \backslash G / N^{-}$).

For an element $\tilde{w} \in W_{a f f}$, written as $w \cdot \check{\nu}$ with $w \in W$, we will denote by ${ }_{\tilde{w}}^{I} \overline{\operatorname{Bun}}_{N^{-}}$ (resp., $I_{\tilde{w}}^{0} \overline{\operatorname{Bun}}_{N^{-}}$) the preimage under $\overline{\mathrm{ev}}_{\check{\nu}}$ of the Schubert cell

$$
B \backslash\left(B \cdot w \cdot N^{-}\right) / N^{-} \subset B \backslash G / N^{-} .
$$

Let ${ }_{\tilde{w}}^{I} \operatorname{Bun}_{N^{-}}$(resp., ${ }_{\tilde{w}}^{I^{0}} \operatorname{Bun}_{N^{-}}$) be the preimage of the same Schubert cell under the map ev $\check{\nu}_{\check{\nu}}:{ }_{\check{\nu}}^{I} \operatorname{Bun}_{N} \rightarrow B \backslash G / N^{-}$. We will denote by $\bar{i}_{\tilde{w}}$ and $i_{\tilde{w}}$ the corresponding locally closed embeddings.

We will denote by $I_{\tilde{w}} \in \operatorname{Perv}\left({ }_{\infty}^{I} \overline{\operatorname{Bun}}_{N^{-}}\right)$the intersection cohomology sheaf on ${ }_{\tilde{w}}^{I} \overline{\mathrm{Bun}}_{N^{-}}$. In other words,

$$
\mathrm{IC}_{\tilde{w}} \simeq\left(\bar{i}_{\check{\nu}}\right)_{! *}\left(\mathrm{IC}_{w, G / B^{-}} \widetilde{\nabla} \mathrm{IC}_{\check{\nu} \mathcal{N}}\right)
$$

in the notation of Proposition 4.2.3. In particular, we see that $I C_{\tilde{w}}$ is an object of $\operatorname{Perv}\left(\mathcal{F} l^{\frac{\infty}{2}}\right)^{I}$, and these sheaves are all the irreducibles of the categories $\operatorname{Perv}\left(\mathcal{F} l^{\frac{\infty}{2}}\right)^{I}$ and $\operatorname{Perv}\left(\mathcal{F} l^{\frac{\infty}{2}}\right)^{0}$.

For $\tilde{w}=w \cdot \check{\mu}$ as above, let us denote by $\nabla_{\tilde{w}}$ and $\Delta_{\tilde{w}}$ the complexes

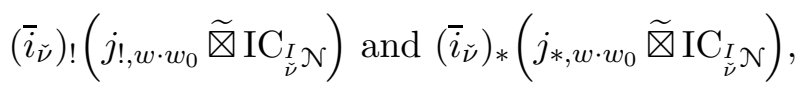

respectively, where $j_{!, w \cdot w_{0}}$ (resp., $j_{*, w \cdot w_{0}}$ ) is the perverse sheaf on $G / B^{-}$corresponding to the same-named perverse sheaf under the isomorphism $G / B^{-} \rightarrow G / B$, given by the right multiplication by $w_{0}$.

According to the above, we can act by objects of $\mathrm{D}(G / B)^{B}$ (resp., $\left.\mathrm{D}(G / B)^{N}\right)$ on objects of $\operatorname{Perv}\left(\mathcal{F l} l^{\frac{\infty}{2}}\right)^{I}$ and obtain complexes, whose cohomologies belong to $\operatorname{Perv}\left(\mathcal{F} l^{\frac{\infty}{2}}\right)^{I}$ (resp., $\left.\operatorname{Perv}\left(\mathcal{F} l^{\frac{\infty}{2}}\right)^{I^{0}}\right)$. Evidently, we have:

$$
j_{!, w_{1}} \star \nabla_{w_{2}} \simeq \nabla_{w_{1} \cdot w_{2}} \text { and } j_{*, w_{1}} \star \Delta_{w_{2}} \simeq \Delta_{w_{1} \cdot w_{2}},
$$

provided that $l\left(w_{1} \cdot w_{2}\right)=l\left(w_{1}\right)+l\left(w_{2}\right)$.

Proposition 4.4.4. Both $\nabla_{\tilde{w}}$ and $\Delta_{\tilde{w}}$ are perverse sheaves.

¿From Proposition 4.2.3 we obtain:

Corollary 4.4.5. Both $\nabla_{\tilde{w}}$ and $\Delta_{\tilde{w}}$ are objects of $\operatorname{Perv}\left(\mathcal{F} l^{\frac{\infty}{2}}\right)^{I}$, 
Proof. Evidently, we have:

$$
\nabla_{\tilde{w}} \simeq\left(\bar{i}_{\tilde{w}}\right) !\left(\mathrm{IC}_{\tilde{w}} \overline{\operatorname{Bun}}_{N^{-}}\right) .
$$

We claim that the morphism $\bar{i}_{\tilde{w}}$ is affine. Clearly, this would imply the proposition. To simplify the notation we will assume that $\check{\nu}=0$; the proof in the general case is the same.

For an element $w \in W$ we can find a weight $\lambda$ and $B$-stable subspaces

$$
{ }^{\prime} V_{w}^{\lambda} \subset V_{w}^{\lambda} \subset V^{\lambda},
$$

with $\operatorname{dim}\left(V_{w}^{\lambda} /{ }^{\prime} V_{w}^{\lambda}\right)=1$, such that a point of $G / B^{-}$, thought of as a quotient line $\ell^{\lambda} \leftarrow V^{\lambda}$, belongs to $B \cdot w \cdot B^{-} / B^{-}$if and only if the composition

$$
{ }^{\prime} V_{w}^{\lambda} \rightarrow V^{\lambda} \rightarrow \ell^{\lambda}
$$

is zero, and $V_{w}^{\lambda} \rightarrow V^{\lambda} \rightarrow \ell^{\lambda}$ is non-zero.

Then, ${ }_{\tilde{w}}^{I} \overline{\operatorname{Bun}}_{N^{-}}$, as a substack of ${ }_{\leq 0}^{I} \overline{\operatorname{Bun}}_{N^{-}}$, corresponds to those $\kappa^{\lambda}$, for which the map

$$
\left({ }^{\prime} V_{w}^{\lambda}\right)_{\mathcal{P}_{G, x}} \rightarrow\left(V_{\mathcal{P}_{G}}^{\lambda}\right)_{x} \rightarrow \mathcal{O}_{x} \simeq \mathbb{C}
$$

is zero, and

$$
\left(V_{w}^{\lambda}\right)_{\mathcal{P}_{G, x}} \rightarrow\left(V_{\mathcal{P}_{G}}^{\lambda}\right)_{x} \rightarrow \mathcal{O}_{x} \simeq \mathbb{C}
$$

is non-zero. Note that $\left(V_{w}^{\lambda}\right)_{\mathcal{P}_{G, x}}$ and $\left({ }^{\prime} V_{w}^{\lambda}\right)_{\mathcal{P}_{G, x}}$ make sense as subspaces of $\left(V_{\mathcal{P}_{G}}^{\lambda}\right)_{x}$, since a part of the data of a point of ${ }_{\infty}^{I} \overline{\mathrm{Bun}}_{N^{-}}$is the reduction of the fiber $\mathcal{P}_{G, x}$ of $\mathcal{P}_{G}$ at $x$ to $B$.

Hence, the closure of ${ }_{\tilde{w}}^{I} \overline{\mathrm{Bun}}_{N^{-}}$is contained in the closed sub-stack of ${ }_{\leq 0}^{I} \overline{\mathrm{Bun}}_{N^{-}}$, consisting of all those points, for which the composition in (38) vanishes. The locus of non-vanishing of (39) is the complement to a Cartier divisor in this closed substack.

4.4.6. We will work with the abelian category $\operatorname{Perv}\left(\mathcal{F} l^{\frac{\infty}{2}}\right)^{I^{0}}$ and its derived category, denoted $\mathrm{D}\left(\mathcal{F} l^{\frac{\infty}{2}}\right)^{I^{0}}$.

By Proposition 4.2 .9 , for $\mathcal{F}_{1}, \mathcal{F}_{2} \in \operatorname{Perv}\left(\mathcal{F} l^{\frac{\infty}{2}}\right)^{I^{0}}$,

$$
\operatorname{Ext}_{\operatorname{Perv}\left(\mathcal{F}^{\frac{\infty}{2}}\right)_{I^{0}}}^{1}\left(\mathcal{F}_{1}, \mathcal{F}_{2}\right) \simeq \operatorname{Ext}_{I^{0} \overline{\operatorname{Bun}}_{N^{-}}^{1}}^{1}\left(\mathcal{F}_{1}, \mathcal{F}_{2}\right)_{T}
$$

where the subscript $T$ stands for the $T$-equivariant category. Hence, the map

$$
\operatorname{Ext}_{\operatorname{Perv}\left(\mathcal{F l} \frac{\infty}{2}\right) I^{0}}^{2}\left(\mathcal{F}_{1}, \mathcal{F}_{2}\right) \rightarrow \operatorname{Ext}_{I_{\infty}^{0} \overline{\operatorname{Bun}}_{N^{-}}^{2}}\left(\mathcal{F}_{1}, \mathcal{F}_{2}\right)_{T}
$$

in injective.

¿From Corollary 4.2.13, and using the fact that each $\underset{\tilde{w}}{I^{0}} \operatorname{Bun}_{N}$ is contractible, we obtain:

Corollary 4.4.7. $\operatorname{Ext}_{\operatorname{Perv}\left(\mathcal{F l} l^{\frac{\infty}{2}}\right)^{I^{0}}}\left(\nabla_{\tilde{w}}, \Delta_{\tilde{w}^{\prime}}\right)=0$ for $i=1,2$ and any $\tilde{w}, \tilde{w}^{\prime} \in W_{\text {aff }}$. 
MODULES OVER THE SMALL QUANTUM GROUP AND SEMI-INFINITE FLAG MANIFOLD 63

Remark. ¿From Corollary 4.4 .7 one can formally deduce that $\operatorname{Ext} t_{\operatorname{Perv}\left(\mathcal{F l} \frac{\infty}{2}\right)^{I^{0}}}^{i}\left(\nabla_{\tilde{w}}, \Delta_{\tilde{w}^{\prime}}\right)$ vanishes for all $i>0$ and any $\tilde{w}, \tilde{w}^{\prime} \in W_{a f f}$. More generally, for $\mathcal{F} \in \operatorname{Perv}\left(\mathcal{F} l^{\frac{\infty}{2}}\right)^{I^{0}}$,

$$
\operatorname{Ext}_{\operatorname{Perv}\left(\mathcal{F l} \frac{\infty}{2}\right)_{I^{0}}}^{i}\left(\nabla_{\tilde{w}}, \mathcal{F}\right) \simeq H^{i}\left(\tilde{w}_{\tilde{w}}^{I^{0}} \operatorname{Bun}_{N},\left.\mathcal{F}\right|_{I_{\tilde{w}}^{0} \operatorname{Bun}_{N}}\right) .
$$

Note that by Proposition 4.2.3, the !-restriction of any $\mathcal{F} \in \operatorname{Perv}\left(\mathcal{F} l^{\frac{\infty}{2}}\right)^{I^{0}}$ to $\underset{\tilde{w}}{I^{0}} \operatorname{Bun}_{N}$ is a complex with constant cohomologies. Since $\tilde{w}_{\tilde{w}}^{I^{0}} \operatorname{Bun}_{N}$ is contractible, $H^{\bullet}\left({ }_{\tilde{w}}^{I^{0}} \operatorname{Bun}_{N}, \underline{\mathbb{C}}\right) \simeq$ $\mathbb{C}$, so, the above expression for $E x t^{i}$ amounts to taking stalks of $\mathcal{F}$ on the stratum ${ }_{\tilde{w}}^{I^{0}} \operatorname{Bun}_{N}$.

4.4.8. The baby Whittaker case. Let $\operatorname{Perv}\left({ }_{\infty}^{1} \overline{\operatorname{Bun}}_{N^{-}}\right)^{N^{-}, \psi}$ be the category of $\left(N^{-}, \psi\right)$ equivariant perverse sheaves on ${ }_{\infty}^{1} \overline{\mathrm{Bun}}_{N^{-}}$. We introduce the category

$$
\operatorname{Perv}\left(\mathcal{F l} l^{\frac{\infty}{2}}\right)^{I^{-}, \psi} \subset \operatorname{Perv}\left({ }_{\infty}^{1} \overline{\operatorname{Bun}}_{N^{-}}\right)_{N^{-}, \psi},
$$

as the full subcategory, consisting of objects, which belong to $\operatorname{Perv}\left(\mathcal{F} l^{\frac{\infty}{2}}\right)^{G^{k}}, k=1$, when regarded merely as objects of $\operatorname{Perv}\left({ }_{\infty}^{1} \overline{\operatorname{Bun}}_{N^{-}}\right)$. This category is stable under extensions by Proposition 4.2.9.

By Proposition 4.2.3, we can produce objects in $\operatorname{Perv}\left(\mathcal{F} l^{\frac{\infty}{2}}\right)^{I^{-}, \psi}$, starting from objects of $\operatorname{Perv}\left(G / B^{-}\right)^{N^{-}, \psi}$. We will denote by $\psi_{G / B^{-}}$the unique irreducible in the latter category, which corresponds to $\psi_{G / B}$ under

$$
\operatorname{Perv}\left(G / B^{-}\right)^{N^{-}, \psi} \simeq \operatorname{Perv}(G / B)^{N^{-}, \psi} .
$$

For $\check{\nu} \in \check{\Lambda}$, set

$$
\mathrm{IC}_{\check{\nu}}^{\psi}:=\left(\bar{i}_{\check{\nu}}\right) ! *\left(\psi_{G / B^{-}} \widetilde{\otimes} \mathrm{IC}_{\check{\nu}} \mathcal{N}\right),
$$

and

$$
\nabla_{\check{\nu}}^{\psi}:=\left(\bar{i}_{\breve{\nu}}\right) !\left(\psi_{G / B^{-}} \widetilde{\otimes} \mathrm{IC}_{\frac{1}{\nu} \mathcal{N}}\right), \Delta_{\check{\nu}}^{\psi}:=\left(\bar{i}_{\breve{\nu}}\right)_{*}\left(\psi_{G / B^{-}} \widetilde{\nabla} \mathrm{IC}_{\check{\nu}} \mathcal{N}\right) .
$$

Since the embedding of the corresponding locally closed subset into ${ }_{\check{\nu}}^{1} \overline{\mathrm{Bun}}_{N^{-}}$is affine (cf. the proof of Proposition 4.4.4), both $\nabla_{\check{\nu}}^{\psi}$ and $\Delta_{\check{\nu}}^{\psi}$ are perverse sheaves, and hence, by Proposition 4.2.3, are objects of $\operatorname{Perv}\left(\mathcal{F l} l^{\frac{\infty}{2}}\right)^{I^{-}, \psi}$. In Sect. 5.3.3 we will prove the following:

Theorem 4.4.9. The canonical maps $\nabla_{\check{\nu}}^{\psi} \rightarrow \mathrm{IC}_{\check{\nu}}^{\psi} \rightarrow \Delta_{\check{\nu}}^{\psi}$ is are isomorphisms.

Thus, the extension of $\psi_{G / B} \widetilde{\otimes} \mathrm{IC}_{\check{\nu}} \overline{\operatorname{Bun}}_{N^{-}}$under $\bar{i}_{\check{\nu}}$ is clean, and $\nabla_{\check{\nu}}^{\psi} \simeq \Delta_{\check{\nu}}^{\psi}$ is irreducible. Hence, the category $\operatorname{Perv}\left(\mathcal{F} l^{\frac{\infty}{2}}\right)^{I^{-}, \psi}$ is semi-simple and equivalent to $\check{T}$-mod.

4.4.10. Let us denote by

$$
\mathrm{Av}_{!, N^{-}, \psi}, \mathrm{Av}_{!, N^{-}, \psi}: \mathrm{D}\left({ }_{\infty}^{1} \overline{\operatorname{Bun}}_{N^{-}}\right) \rightarrow \mathrm{D}\left({ }_{\infty}^{1} \overline{\operatorname{Bun}}_{N^{-}}\right)^{I^{-}, \psi}
$$

the functors, which are left and right adjoint, respectively, to $\mathrm{D}\left({ }_{\infty}^{1} \overline{\mathrm{Bun}}_{N^{-}}\right)^{I^{-}, \psi} \rightarrow$ $\mathrm{D}\left({ }_{\infty}^{k} \overline{\mathrm{Bun}}_{N^{-}}\right)$. As in Proposition 2.2.5 we obtain: 
Lemma 4.4.11. There exists an isomorphism of functors

$$
\left.\left.\operatorname{Av}_{!, N^{-}, \psi}[-\operatorname{dim}(\mathfrak{n})]\right|_{\mathrm{D}\left(I_{\infty}^{0} \overline{\operatorname{Bun}}_{N^{-}}\right)} \rightarrow \operatorname{Av}_{*, N^{-}, \psi}[\operatorname{dim}(\mathfrak{n})]\right|_{\mathrm{D}\left({ }_{\infty}^{I^{0}} \overline{\operatorname{Bun}}_{N^{-}}\right)} \cdot
$$

Moreover, the resulting functor $\mathrm{Av}_{N^{-}, \psi}: \mathrm{D}\left({ }_{\infty}^{I^{0}} \overline{\mathrm{Bun}}_{N^{-}}\right) \rightarrow \mathrm{D}\left({ }_{\infty}^{1} \overline{\operatorname{Bun}}_{N^{-}}\right)^{I^{-}, \psi}$ is exact.

Let us call an object of $\operatorname{Perv}\left(\mathcal{F} l^{\frac{\infty}{2}}\right)^{I^{0}}$ partially integrable if all of its irreducible subquotients are of the form $\mid \mathrm{IC}_{w \cdot \check{\nu}}, w \neq w_{0}$. Thus, the only irreducibles, that are not partially integrable are $\mathrm{IC}_{w_{0} \cdot \check{\nu}}$. Let us denote by ${ }^{f} \operatorname{Perv}\left(\mathcal{F} l^{\frac{\infty}{2}}\right)^{I^{0}}$ the resulting quotient abelian category.

The following is parallel to Proposition 2.2.7.

\section{Proposition 4.4.12.}

(1) The functor

$$
\operatorname{Av}_{I^{-}, \psi}: \operatorname{Perv}\left(\mathcal{F} l^{\frac{\infty}{2}}\right)^{I^{0}} \rightarrow \operatorname{Perv}\left(\mathcal{F} l^{\frac{\infty}{2}}\right)^{I^{-}, \psi}
$$

factors through ${ }^{f} \operatorname{Perv}\left(\mathcal{F} l^{\frac{\infty}{2}}\right) I^{0}$.

(2) The resulting functor

$$
{ }^{f} \operatorname{Perv}\left(\mathcal{F} l^{\frac{\infty}{2}}\right)^{I^{0}} \rightarrow \operatorname{Perv}\left(\mathcal{F} l^{\frac{\infty}{2}}\right)^{I^{-}, \psi}
$$

is faithful.

Proof. To prove the first statement we have to show that $\operatorname{Av}_{I^{-}, \psi}\left(\mathrm{IC}_{w \cdot \check{\nu}}\right)=0$ for $w \neq$ $w_{0}$. This is nearly evident: such an irreducible is a pull-back from the quotient stack $P_{\imath} \backslash 1 \overline{\mathrm{Bun}}_{N^{-}}$, where $P_{\imath}$ is some sub-minimal parabolic in $G$. Our assertion follows from the fact that the direct image of $\psi_{G / B}$ under $G / B \rightarrow G / P_{\imath}$ vanishes.

To prove the second statement, it suffices to show that

$$
\operatorname{Av}_{I^{-}, \psi}\left(\mathrm{IC}_{w_{0} \cdot \check{\nu}}\right) \simeq \mathrm{IC}_{\check{\nu}}^{\psi} .
$$

We know that the left-hand side is a perverse sheaf, and the isomorphism over the open part of the support, namely ${ }_{\nu}^{1} \overline{\mathrm{Bun}}_{N^{-}}$, is evident. The fact that the left-hand side is a Goresky-MacPherson extension from this sub-stack follows from the exactness of the functor $\operatorname{Av}_{I^{-}, \psi}$, and the fact that it commutes with all $\bar{i}_{\check{\nu}^{\prime}}^{*}$ and $\bar{i}_{\check{\nu}^{\prime}}$.

\section{Corollary 4.4.13.}

(1) The kernel of $\nabla_{w_{0} \cdot \check{\nu}} \rightarrow \mid \mathrm{IC}_{w_{0} \cdot \check{\nu}}$ is partially integrable.

(2) $\mathrm{IC}_{w_{0} \cdot \check{\nu}}$ is the cosocle of $\Delta_{\check{\nu}}$ and socle of $\nabla_{\check{\nu}}$.

(3) For any $w \in W, I C_{w_{0} \cdot \check{\nu}}$ is the only non-partially integrable constituent of $\nabla_{w \cdot \check{\nu}}$.

Proof. Evidently, we have

$$
\operatorname{Av}_{I^{-}, \psi}\left(\nabla_{w_{0} \cdot \check{\nu}}\right) \simeq \nabla_{\check{\nu}}^{\psi}
$$

Combining this with Proposition 4.4.12 and Theorem 4.4.9, we arrive to the assertion of point (1). Point (3) follows from point (1) by (37). Finally, point (2) follows from point (1) in the same way as in the proof of Proposition 2.3.2. 
We will now introduce one more object of $\operatorname{Perv}\left(\mathcal{F} l^{\frac{\infty}{2}}\right)^{I^{0}}$. For $\check{\nu} \in \check{\Lambda}$ set

$$
\Pi_{!, \check{\nu}}:=\left(\bar{i}_{\check{\nu}}\right) !\left(\Xi \widetilde{\nabla} \mathrm{IC}_{\check{\nu}} \overline{\operatorname{Bun}}_{N^{-}}\right) \text {and } \Pi_{*, \check{\nu}}:=\left(\bar{i}_{\check{\nu}}\right)_{*}\left(\Xi \widetilde{\nabla} \operatorname{IC}_{\check{\nu}} \overline{\operatorname{Bun}}_{N^{-}}\right),
$$

where $\Xi$ is the perverse sheaf on $N \backslash G / B^{-}$, corresponding to the same-named perverse sheaf on $G / B$.

Theorem 4.4.14. The canonical map $\boldsymbol{\Pi}_{!, \check{\nu}} \rightarrow \boldsymbol{\Pi}_{*, \check{\nu}}$ is an isomorphism.

Proof. Consider the convolution with $\Xi$ as a functor $\operatorname{Perv}\left(\mathcal{F} l^{\frac{\infty}{2}}\right)^{I} \rightarrow \operatorname{Perv}\left(\mathcal{F} l^{\frac{\infty}{2}}\right)^{I^{0}}$. As usual, this functor annihilates all partially integrable objects.

Evidently,

$$
\Pi_{!, \check{\nu}} \simeq \Xi \star \nabla_{w_{0}, \check{\nu}} \text { and } \Pi_{! * \check{\nu}} \simeq \Xi \star \Delta_{w_{0}, \check{\nu}}
$$

Our assertion follows now from Corollary 4.4.13, which implies that the cone of the map $\nabla_{w_{0}, \check{\nu}} \rightarrow \Delta_{w_{0}, \check{\nu}}$ is partially integrable.

4.4.15. We will now establish the following fact, parallel to Proposition 3.2.6(2):

Proposition 4.4.16. For $\check{\nu} \in \check{\Lambda}$ there exists a non-zero map

$$
\Delta_{w_{0} \cdot \check{\nu}} \rightarrow \mathrm{IC}_{\check{\nu}-2 \check{\rho}},
$$

where $\mid \mathrm{IC}_{\check{\nu}-2 \check{\rho}} \in \operatorname{Perv}\left(\mathcal{F} l^{\frac{\infty}{2}}\right)^{G[[t]]}$ is thought of as an object of $\operatorname{Perv}\left(\mathcal{F} l^{\frac{\infty}{2}}\right)^{I}$.

Proof. As in the proof of Proposition 2.3.4, we have the functor

$$
\mathrm{Av}_{!, G / B}: \mathrm{D}\left({ }_{\infty}^{I} \overline{\operatorname{Bun}}_{N^{-}}\right) \rightarrow \mathrm{D}\left({ }_{\infty} \overline{\operatorname{Bun}}_{N^{-}}\right),
$$

left adjoint to the forgetful functor. By definition,

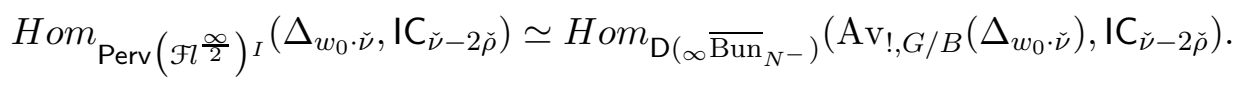

However, since $G / B$ is proper,

$$
\operatorname{Av}_{!, G / B}\left(\Delta_{w_{0} \cdot \check{\nu}}\right) \simeq\left(\bar{i}_{\check{\nu}}\right)_{*}\left(\mathrm{IC}_{\check{\nu}} \overline{\operatorname{Bun}}_{N^{-}}\right)[\operatorname{dim}(\mathfrak{n})] .
$$

Hence, the assertion of the proposition follows from Corollary 4.3.5.

\section{Convolution}

\subsection{Definition of convolution.}

5.1.1. Consider the Hecke stack for $G$ at $x$ :

$$
\operatorname{Bun}_{G} \stackrel{\overleftarrow{h}_{G}}{\longleftarrow} \mathcal{H}_{G, x} \stackrel{\vec{h}_{G}}{\longrightarrow} \operatorname{Bun}_{G}
$$

and for two integers $k_{1}, k_{2}$ let ${ }^{k_{1}, k_{2}} \mathcal{H}_{G, x}$ denote its base change with respect to

$$
{ }^{k_{1}} \operatorname{Bun}_{G} \times{ }^{k_{2}} \operatorname{Bun}_{G} \rightarrow \operatorname{Bun}_{G} \times \operatorname{Bun}_{G} .
$$

By a slight abuse of notation we will continue to denote by $\overleftarrow{h}_{G}, \vec{h}_{G}$ the projections of ${ }^{k_{1}, k_{2}} \mathcal{H}_{G, x}$ on ${ }^{k_{1}} \operatorname{Bun}_{G}$ and ${ }^{k_{2}} \operatorname{Bun}_{G}$, respectively. 
We can regard ${ }^{k_{1}, k_{2}} \mathcal{H}_{G, x}$ over ${ }^{k_{2}} \operatorname{Bun}_{G}$ as the space associated with the canonical $G^{k_{2}}$-torsor $\mathcal{G}_{x}^{k_{2}}$ over ${ }^{k_{2}} \operatorname{Bun}_{G}$ and the $G^{k_{2}}$-space $G((t)) / G^{k_{1}}$ :

$$
{ }^{k_{1}, k_{2}} \mathcal{H}_{G, x} \simeq G((t)) / G^{k_{1}} \stackrel{G^{k_{2}}}{\times} \mathcal{G}_{x}^{k_{2}} .
$$

We also have a symmetric picture:

$$
k_{1}, k_{2} \mathcal{H}_{G, x} \simeq G((t)) / G^{k_{2}} \stackrel{G^{k_{1}}}{\times} \mathcal{G}_{x}^{k_{1}} .
$$

Recall now that there exists a canonical equivalence of derived categories

$$
\mathcal{S} \mapsto \mathcal{S}^{o p}: \mathrm{D}_{G^{k_{1}}}\left(G((t)) / G^{k_{2}}\right) \simeq \mathrm{D}_{G^{k_{2}}}\left(G((t)) / G^{k_{1}}\right) .
$$

It is defined as follows.

First of all, it is clear that $G^{k_{1}}$-invariant sub-schemes of $G((t)) / G^{k_{2}}$ are in bijection with $G^{k_{2}}$-invariant sub-schemes in $G^{k_{1}} \backslash G((t))$. For $\mathcal{S} \in \mathrm{D}_{G^{k_{1}}}\left(G((t)) / G^{k_{2}}\right)$, let $y$ be the corresponding finite-dimensional sub-scheme of $G^{k_{1}} \backslash G((t))$. There exists an integer $k_{1}^{\prime}>>0$, such that if we denote by $y^{\prime}$ the preimage of $y$ in $G^{k_{1}^{\prime}} \backslash G((t))$, the map $\mathrm{y}^{\prime} \rightarrow G((t)) / G^{k_{2}}$ is well-defined. The pull-back $\mathcal{S}^{\prime}$ of $\mathcal{S}$ to $\mathrm{y}^{\prime}$ is an $G^{k_{1}} / G^{k_{1}^{\prime}}$-equivariant, and, hence, descends to a well-defined $G^{k_{2}}$-equivariant object of $\mathrm{D}\left(G^{k_{1}} \backslash G((t))\right)$.

Finally, the desired functor is obtained by applying the inversion on $G((t))$.

5.1.2. As in $[\mathrm{BG}]$ we have a commutative diagram, in which both squares are Cartesian

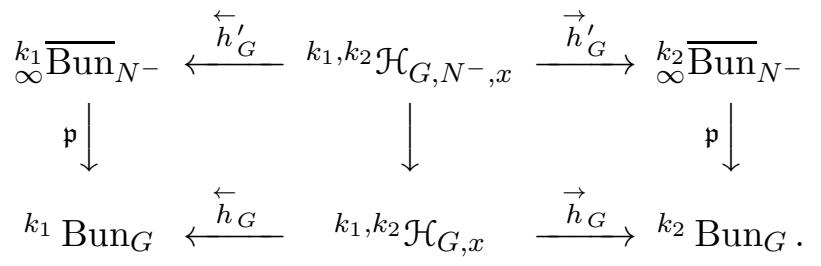

For a complex $\mathcal{F}$ on ${ }_{\infty}^{k_{2}} \overline{\mathrm{Bun}}_{N^{-}}$and a $G^{k_{1}}$-equivariant complex $\mathcal{S}$ on $G((t)) / G^{k_{2}}$ let $\mathcal{S}^{o p} \widetilde{\nabla} \mathcal{F}$ be the corresponding complex on ${ }^{k_{1}, k_{2}} \mathcal{H}_{G, N^{-}, x}$. We set

$$
\mathcal{S}^{*} \mathcal{F}:=\left(\overleftarrow{h_{G}^{\prime}}\right)_{*}\left(\mathcal{S}^{o p} \widetilde{\otimes} \mathcal{F}\right), \mathcal{S} \stackrel{!}{\star} \mathcal{F}:=\left(\overleftarrow{h_{G}^{\prime}}\right)_{!}\left(\mathcal{S}^{o p} \widetilde{\otimes} \mathcal{F}\right) \in \mathrm{D}\left({ }_{\infty}^{k_{1}} \overline{\mathrm{Bun}}_{N^{-}}\right)
$$

Evidently, when $k_{1}=k_{2}=k$, and $\mathcal{S}$ is supported on $G[[t]] / G^{k} \subset G((t)) / G^{k}$, we arrive to the functors discussed in Sect. 4.4.1.

The following is straightforward from the definitions:

Lemma 5.1.3. For $\mathcal{S} \in \mathrm{D}_{G^{k_{1}}}\left(G((t)) / G^{k_{2}}\right)$ the functor

$$
\mathcal{F} \mapsto \mathcal{S} \stackrel{!}{\star} \mathcal{F}: \mathrm{D}\left(k_{\infty}^{k_{2}} \overline{\mathrm{Bun}}_{N^{-}}\right) \rightarrow \mathrm{D}\left({ }_{\infty}^{k_{1}} \overline{\mathrm{Bun}}_{N^{-}}\right)
$$

is the left adjoint of

$$
\mathcal{F}^{\prime} \mapsto \mathbb{D}\left(\mathcal{S}^{o p}\right) \stackrel{*}{\star} \mathcal{F}^{\prime}: \mathrm{D}\left({ }_{\infty}^{k_{1}} \overline{\operatorname{Bun}}_{N^{-}}\right) \rightarrow \mathrm{D}\left({ }_{\infty}^{k_{2}} \overline{\operatorname{Bun}}_{N^{-}}\right) .
$$

The above picture admits the following variants. First, we can replace the equivariance condition on $\mathcal{S} \in \mathrm{D}\left(G((t)) / G^{k_{2}}\right)$ with respect to $G^{k_{1}}$ by that of $I^{0}, I$ or $\left(I^{-}, \psi\right)$. 
In the case the target will be the corresponding category $\mathrm{D}\left({ }_{\infty}^{1} \overline{\mathrm{Bun}}_{N^{-}}\right), \mathrm{D}\left({ }_{\infty}^{I} \overline{\mathrm{Bun}}_{N^{-}}\right)$or $\mathrm{D}\left({ }_{\infty}^{1} \overline{\mathrm{Bun}}_{N^{-}}\right)^{N^{-}, \psi}$.

Secondly, instead $\mathrm{D}\left(G((t)) / G^{k_{2}}\right)$ we can consider $\mathrm{D}\left(\mathrm{Fl}_{G}\right)$ or $\mathrm{D}\left(\mathrm{Gr}_{G}\right)$. We obtain the convolution functors

$\mathrm{D}\left(\mathrm{Fl}_{G}\right)^{G^{k}} \times \mathrm{D}\left({ }_{\infty}^{I} \overline{\mathrm{Bun}}_{N^{-}}\right) \rightarrow \mathrm{D}\left({ }_{\infty}^{k} \overline{\operatorname{Bun}}_{N^{-}}\right)$and $\mathrm{D}\left(\mathrm{Gr}_{G}\right)^{G^{k}} \times \mathrm{D}\left({ }_{\infty} \overline{\mathrm{Bun}}_{N^{-}}\right) \rightarrow \mathrm{D}\left({ }_{\infty}^{k} \overline{\operatorname{Bun}}_{N^{-}}\right)$.

In both these cases, the ${ }^{*}$-convolution coincides with the !-convolution, since $\mathrm{Fl}_{G}$ and $\mathrm{Gr}_{G}$ are ind-proper. We will denote the resulting functor simply by $\star$. Here again, the equivariance condition with respect to $G^{k}$ can be replaced by any of $I^{0}$-, $I$ - or $\left(I^{-}, \psi\right)$-equivariance conditions.

5.1.4. We will now show that the convolution functors essentially preserve our category $\operatorname{Perv}\left(\mathcal{F} l^{\frac{\infty}{2}}\right)$.

Proposition 5.1.5. If $\mathcal{F} \in \operatorname{Perv}\left(\mathcal{F} l^{\frac{\infty}{2}}\right)^{G^{k_{2}}}$, then the perverse cohomologies of both $\mathcal{S}{ }^{*} \mathcal{F}$ and $\mathcal{S} \stackrel{!}{\star} \mathcal{F}$ belong to $\operatorname{Perv}\left(\mathcal{F} l^{\frac{\infty}{2}}\right)^{G^{k_{1}}}$.

The rest of this subsection is devoted to the proof of this proposition. First, let us notice that if $\mathcal{F}$ satisfies condition $(1)$, then so do the complexes $\left(\overleftarrow{h}_{G}^{\prime}\right)_{*}\left(\mathcal{S}^{o p} \widetilde{\otimes} \mathcal{F}\right)$ and $\left(\overleftarrow{h^{\prime}}{ }_{G}\right) !\left(\mathcal{S}^{o p} \widetilde{\otimes} \mathcal{F}\right)$. Hence, by Lemma 4.2 .5 and Proposition 4.2.6, these complexes satisfy the weak factorization property. Hence, to show that their perverse cohomologies satisfy the full factorization property, it is enough to show that their pull-backs to

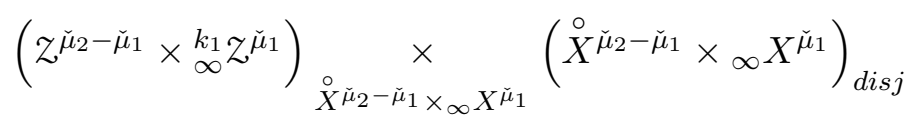

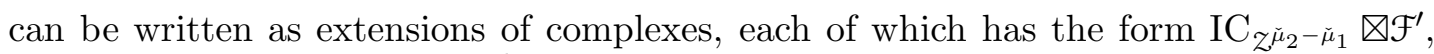
where $\mathcal{F}^{\prime}$ is some complex on $k_{\infty} Z^{\check{\mu}_{1}}$.

Let us denote by $y^{\check{\mu}}$ the Cartesian product

$$
{ }_{\infty}^{k_{1}} \mathcal{Z}^{\check{\mu}} \underset{\infty}{k_{1} \frac{\times}{\operatorname{Bun}_{N^{-}}}}{ }^{k_{1}, k_{2}} \mathcal{H}_{G, N, x}
$$

As in Lemma 4.1.5, we have a canonical isomorphism

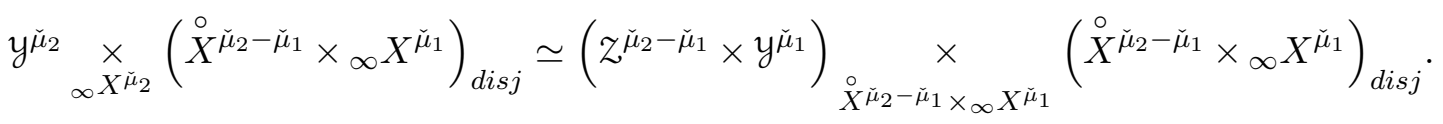

We claim that the pull-back under

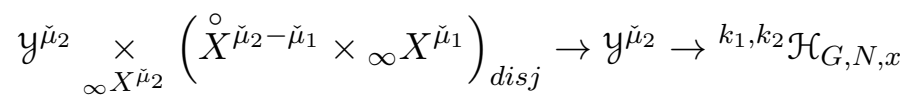

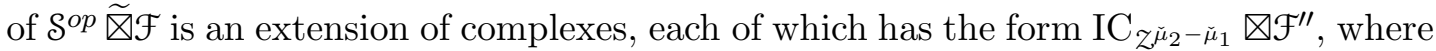
$\mathcal{F}^{\prime \prime}$ is some complex on $y^{\check{\mu}_{1}}$. This would clearly imply our assertion.

Note that $y^{\check{\mu}}$ can be represented as a union of locally closed sub-stacks $\check{\nu} y^{\check{\mu}}$ for $\check{\nu} \in \check{\Lambda}$, where a point $\left(\mathcal{P}_{G},\left\{\kappa^{\lambda}\right\},\left\{\kappa^{\lambda,-}\right\}\right)$ belongs to $\check{\nu} y \check{\mu}$ if and only if each $\kappa^{\lambda,-}$ has a pole of order $\langle\lambda, \check{\nu}\rangle$ at $x$. 
Note that we have a natural map $\check{\nu}_{\check{\nu}} \mathrm{y}_{\infty} \mathrm{k}_{2} z^{\check{\mu}+\check{\nu}}$, that covers the map

$$
\vec{h}_{G}^{\prime}:{ }^{k_{1}, k_{2}} \mathcal{H}_{G, N, x} \rightarrow{ }_{\infty}^{k_{2}} \overline{\operatorname{Bun}}_{N^{-}} .
$$

Moreover, the diagram

$$
\begin{aligned}
& \check{\nu} y^{\check{\mu}_{2}} \underset{\infty X^{\check{\mu}_{2}}}{\times}\left(\stackrel{\circ}{X^{\check{\mu}_{2}-\check{\mu}_{1}}} \times_{\infty} X^{\check{\mu}_{1}}\right)_{d i s j} \longrightarrow Z^{\check{\mu}_{2}-\check{\mu}_{1}} \times_{\check{\nu}} y^{\check{\mu}_{1}} \\
& \downarrow \\
& k_{2} z^{\check{\mu}_{2}+\check{\nu}} \underset{\infty X^{\check{\mu}_{2}+\check{\nu}}}{\times}\left(\stackrel{\circ}{X^{\check{\mu}_{2}-\check{\mu}_{1}}} \times_{\infty} X^{\check{\mu}_{1}+\check{\nu}}\right)_{d i s j} \longrightarrow z^{\check{\mu}_{2}-\check{\mu}_{1}} \times \underset{\infty}{k_{2}} z^{\check{\mu}_{1}+\check{\nu}}
\end{aligned}
$$

is commutative. Hence, our assertion follows from condition (2) imposed on $\mathcal{F}$.

It remains to show that the perverse cohomologies of $\mathcal{S}^{*} \mathcal{F}$ and $\mathcal{S} \stackrel{!}{\star} \mathcal{F}$ satisfy condition (3). Since we have to check an equivariance condition with respect to a unipotent group-scheme, it is enough to show that their pull-backs to ${ }_{\infty}^{k_{1}} z^{\check{\mu}}$ can be written as extensions of complxes satisfying this equivariance condition. This follows in the same way as above, by sub-dividing the stack $y \check{\mu}$ into the locally closed substacks $\check{\nu} y \check{\mu}$.

\subsection{Exactness and smallness.}

\subsubsection{Consider the convolution functor}

$$
\mathrm{D}\left(\mathrm{Gr}_{G}\right)^{G^{k}} \times \operatorname{Perv}\left(\mathcal{F} l^{\frac{\infty}{2}}\right)^{G[[t]]} \rightarrow \mathrm{D}\left({ }_{\infty}^{k} \overline{\operatorname{Bun}}_{N^{-}}\right) .
$$

Since $\operatorname{Perv}\left(\mathcal{F} l^{\frac{\infty}{2}}\right)^{G[[t]]}$ is semi-simple, it is enough to evaluate the above functor on the objects of the form $I C_{\check{\nu}}, \check{\nu} \in \check{\Lambda}$.

Theorem 5.2.2. The functor

$$
\mathcal{S} \mapsto \mathcal{S} \star \mathrm{IC}_{\check{\nu}}: \mathrm{D}\left(\mathrm{Gr}_{G}\right)^{G^{k}} \rightarrow \mathrm{D}\left({ }_{\infty}^{k} \overline{\operatorname{Bun}}_{N^{-}}\right) .
$$

is exact.

Proof. Since the situation is self-dual with respect to the Verdier duality, it is sufficient to show that for $\mathcal{S} \in \operatorname{Perv}\left(\operatorname{Gr}_{G}\right)^{G^{k}}$, the convolution $\mathcal{S} \star I C_{\check{\nu}}$ is supported in non-positive cohomological degrees. For that it is sufficient to show that $\bar{i}_{\breve{\mu}}^{*}\left(\mathcal{S} \star I C_{\check{\nu}}\right)$ is supported in non-positive cohomological degrees for every $\check{\mu} \in \check{\Lambda}$.

Consider the preimage $\left(\overleftarrow{h}{ }_{G}^{\prime}\right)^{-1}\left({ }_{\breve{\mu}}^{k} \overline{\operatorname{Bun}}_{N^{-}}\right) \subset^{k, 0} \mathcal{H}_{G, N, x}$. It admits a decomposition into locally closed pieces

$$
\left(\overleftarrow{h}_{G}^{\prime}\right)^{-1}\left({ }_{\check{\mu}}^{k} \overline{\operatorname{Bun}}_{N^{-}}\right) \cap\left(\vec{h}_{G}^{\prime}\right)^{-1}\left(\check{\check{\mu}}^{\prime} \overline{\operatorname{Bun}}_{N^{-}}\right) \cap{ }^{k, 0} \mathcal{H}_{G, N, x}^{\check{\lambda}}
$$

for $\check{\mu}^{\prime} \in \check{\Lambda}$ and $\check{\lambda} \in \check{\Lambda}^{+}$, where ${ }^{k, 0} \mathcal{H}_{G, N, x}^{\check{\lambda}}$ is the preimage of the corresponding locally closed sub-stack in $\mathcal{H}_{G, x}$.

The statement of the theorem would follow once we prove the following:

(1) The dimension of fibers of the map

$$
\overleftarrow{h}_{G}^{\prime}:\left(\overleftarrow{h}_{G}^{\prime}\right)^{-1}\left({ }_{\breve{\mu}}^{k} \overline{\operatorname{Bun}}_{N^{-}}\right) \cap\left(\vec{h}_{G}^{\prime}\right)^{-1}\left(\check{\mu}^{\prime} \overline{\operatorname{Bun}}_{N^{-}}\right) \cap{ }^{k, 0} \mathcal{H}_{G, N, x}^{\check{\lambda}} \rightarrow{ }_{\breve{\mu}}^{k} \overline{\operatorname{Bun}}_{N^{-}}
$$



is $\leq\left\langle\check{\mu}^{\prime}-\check{\mu}+\check{\lambda}, \rho\right\rangle$.

(2) The *-restriction of $\mathcal{S}^{o p} \widetilde{\nabla} \mathbf{I C}_{\check{\nu}}$ to $\left(\overleftarrow{h}_{G}^{\prime}\right)^{-1}\left({ }_{\breve{\mu}}^{k} \overline{\operatorname{Bun}}_{N^{-}}\right) \cap\left(\vec{h}_{G}^{\prime}\right)^{-1}\left(\check{\check{\mu}}^{\prime} \overline{\operatorname{Bun}}_{N^{-}}\right) \cap \cap^{k, 0} \mathcal{H}_{G, N, x}^{\check{\lambda}}$ lives in the cohomological degrees $\leq-\left\langle\check{\mu}^{\prime}-\check{\mu}+\check{\lambda}, \rho\right\rangle$.

The first assertion follows from the identification of the locally closed substack from (41), projecting to $\underset{\check{\mu}}{k} \overline{\mathrm{Bun}}_{N^{-}}$by means of $\overleftarrow{h}_{G}^{\prime}$, with

$$
\left(\operatorname{Gr}_{G}^{\check{\lambda}} \cap N^{-}((t)) \cdot\left(\check{\mu}-\check{\mu}^{\prime}\right)\right)^{N^{-}[[t]]}{ }^{k} \underset{\mu}{\mathcal{N}},
$$

where ${ }_{\breve{\mu}}^{k} \mathcal{N}$ is the $N^{-}[[t]]$-torsor over ${ }_{\breve{\mu}}^{k} \overline{\mathrm{Bun}}_{N^{-}}$introduced before.

To prove the second assertion let us view the locally closed sub-stack of (41) projecting to $\check{\mu}^{\prime} \overline{\mathrm{Bun}}_{N^{-}}$by means of $\vec{h}_{G}^{\prime}$; it identifies with

$$
p_{k}^{-1}\left(\operatorname{Gr}_{G}^{-w_{0}(\check{\lambda})} \cap N^{-}((t)) \cdot\left(\check{\mu}^{\prime}-\check{\mu}\right)\right)^{N^{-}[[t]]}{ }_{\check{\mu}^{\prime}} \mathcal{N},
$$

where $p_{k}$ is the projection $G((t)) / G^{k} \rightarrow \mathrm{Gr}_{G}$.

The ${ }^{*}$-restriction of $\mathcal{S}^{o p} \widetilde{\nabla} \mid C_{\check{\nu}}$ to it identifies with

$$
\left.\mathcal{S}^{o p}\right|_{p_{k}^{-1}\left(\operatorname{Gr}_{G}^{-w_{0}(\check{\lambda})} \cap N^{-}((t)) \cdot\left(\check{\mu}^{\prime}-\check{\mu}\right)\right)} \widetilde{\otimes}\left|C_{\check{\nu}}\right|_{\check{\mu}^{\prime}} \overline{\operatorname{Bun}}_{N^{-}} .
$$

Hence, it is enough to show that the ${ }^{*}$-restriction of $\mathcal{S}^{o p}$ to

$$
p_{k}^{-1}\left(\operatorname{Gr}_{G}^{-w_{0}(\check{\lambda})} \cap N^{-}((t)) \cdot\left(\check{\mu}^{\prime}-\check{\mu}\right)\right)
$$

lives in the cohomological degrees $\leq-\left\langle\check{\mu}^{\prime}-\check{\mu}+\check{\lambda}, \rho\right\rangle$.

First, the restriction to $p_{k}^{-1}\left(\mathrm{Gr}_{G}^{-w_{0}(\check{\lambda})}\right)$ lives in non-positive degrees, since $\mathcal{S}$ was assumed perverse. By assumption, this complex is $G[[t]]$-equivariant, and hence, universally locally acyclic over $\operatorname{Gr}_{G}^{-w_{0}(\check{\lambda})}$, since the latter is a $G[[t]]$-homogeneous space. Since

$$
\operatorname{codim}\left(\operatorname{Gr}_{G}^{-w_{0}(\check{\lambda})} \cap N^{-}((t)) \cdot\left(\check{\mu}^{\prime}-\check{\mu}\right), \mathrm{Gr}_{G}^{-w_{0}(\check{\lambda})}\right) \geq\left\langle\check{\mu}^{\prime}-\check{\mu}+\check{\lambda}, \rho\right\rangle,
$$

our assertion follows.

5.2.3. Convolution in the spherical case. We will now study a particular case of the above situation, when the functor we consider is:

$$
\operatorname{Sph}_{G} \times \operatorname{Perv}\left(\mathcal{F} l^{\frac{\infty}{2}}\right)^{G[[t]]} \rightarrow \operatorname{Perv}\left(\mathcal{F} l^{\frac{\infty}{2}}\right)^{G[[t]]} .
$$

Proposition 5.2.4. For $V \in \operatorname{Rep}(\check{G})$ and $\check{\nu} \in \check{\Lambda}$, there exists a canonical isomorphism

$$
\mathcal{V} \star \mathrm{I} \mathrm{C}_{\check{\nu}} \simeq \underset{\check{\mu}}{\oplus \mathrm{IC}} \mathrm{C}_{\check{\nu}+\check{\mu}} \otimes \underline{V}(\check{\mu}) .
$$


Moreover, for $V, U \in \operatorname{Rep}(\check{G})$, the diagram

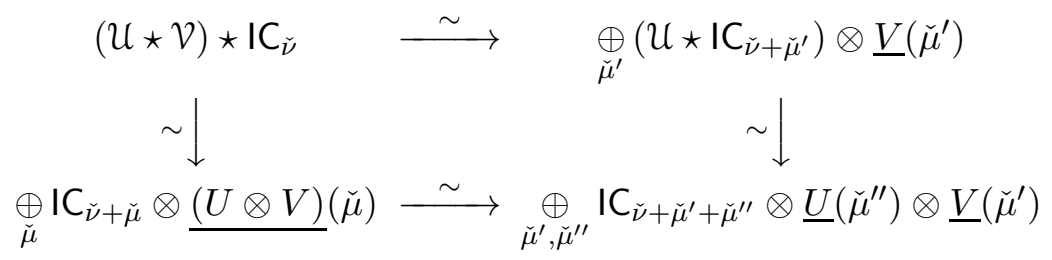

commutes.

Before giving the proof let us recall that the for $V \in \operatorname{Rep}(\check{G})$ and the corresponding object $\mathcal{V} \in \mathrm{Sph}_{G}$, we have a canonical isomorphism

$$
V(\check{\mu}) \simeq H_{c}^{-\langle 2 \rho, \check{\mu}\rangle}\left(N^{-}((t)) \cdot \check{\mu},\left.\mathcal{V}\right|_{N^{-}((t)) \cdot \check{\mu}}\right) .
$$

Proof. Note first that the result of the convolution $\mathcal{V}_{\star} \mid C_{\check{\nu}}$ is an object of $\operatorname{Perv}\left(\mathcal{F} l^{\frac{\infty}{2}}\right)^{G[[t]]}$, and hence, is semi-simple. (Alternatively, semi-simplicity follows from the decomposition theorem, since every $\mathcal{V} \in \mathrm{Sph}_{G}$ is a direct sum of intersection cohomology sheaves.)

By the proof of Theorem 5.2.2,

$$
\operatorname{Hom}\left(\mathrm{IC}_{\check{\nu}+\check{\mu}}, \mathcal{V} \star \mathrm{IC} \check{\check{\nu}}\right) \simeq H_{c}^{-\langle 2 \rho, \check{\mu}\rangle}\left(N^{-}((t)) \cdot \check{\mu},\left.\mathcal{V}\right|_{N^{-}}((t)) \cdot \check{\mu}\right),
$$

which is exactly the expression that appears in (43).

The second assertion of the proposition follows from the definition of the structure of the tensor functor on $V \mapsto \mathcal{V}: \operatorname{Rep}(\check{G}) \rightarrow \mathrm{Sph}_{G}$, cf. [MV] or [BG1].

The commutativity of the following two diagrams also follows from (43):

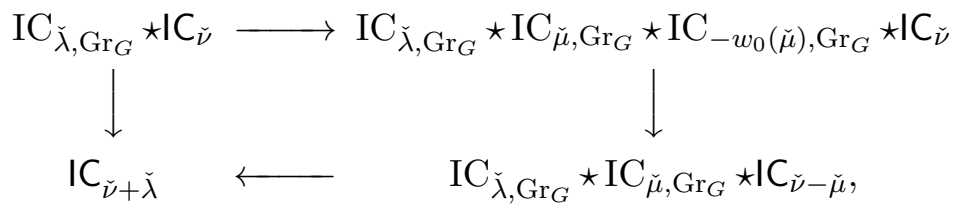

where the left vertical arrow comes from taking the direct summand corresponding to $\underline{V}^{\check{\lambda}}(\check{\lambda})$, and the right vertical arrow comes from taking the summand corresponding to $\underline{V}^{-w_{0}(\check{\mu})}(-\check{\mu})$.

For the following diagram $V$ is an object of $\operatorname{Rep}(\check{G})$ and $\check{\lambda}$ is a coweight large compared to $V$ :

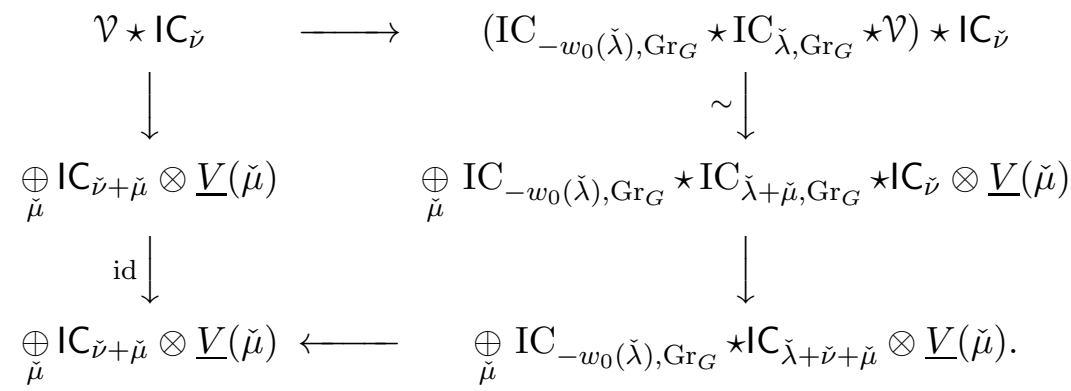

\subsection{Convolution with $\operatorname{Perv}\left(\mathrm{Gr}_{G}\right)^{I}$.}


MODULES OVER THE SMALL QUANTUM GROUP AND SEMI-INFINITE FLAG MANIFOLD 71

5.3.1. We will now consider the convolution functor

$$
\operatorname{Perv}_{G[[t]]}\left(\mathrm{Fl}_{G}\right) \times \operatorname{Perv}\left(\mathcal{F} l^{\frac{\infty}{2}}\right)^{G[[t]]} \rightarrow \operatorname{Perv}\left(\mathcal{F} l^{\frac{\infty}{2}}\right)^{I} .
$$

Recall the objects $\mathcal{L}^{w} \in \operatorname{Perv}\left(\operatorname{Gr}_{G}\right)^{I}$ defined for $w \in W$ We will prove:

Theorem 5.3.2. If $\mathcal{L}^{w}=\mathrm{IC}_{w \cdot \check{\lambda}, \mathrm{Gr}_{G}}$, then

$$
\mathcal{L}^{w} \star I C_{\check{\nu}} \simeq I C_{w \cdot(\check{\lambda}+\check{\nu})}
$$

The rest of this sub-section is devoted to the proof of this theorem. We will retrace the argument proving Theorem 5.2.2 and show that the map defining $\mathcal{L}^{w} \star I C_{\breve{\nu}}$ is small (vs. semi-small).

First, to calculate the top $\left(=0\right.$-th) cohomology of $\mathcal{L}^{w} \star \mathrm{IC}_{\check{\nu}}$ we only need to consider the locally closed sub-stack of ${ }^{I, 0} \mathcal{H}_{G, N^{-}, x}$ isomorphic to

$$
\mathbb{O}_{w}{ }^{N^{-}[[t]]}{ }_{\check{\nu}}^{I} \mathcal{N}
$$

and the constant perverse sheaf on it, where $\mathbb{O}_{\tilde{w}}$ is the open $G[[t]]$-orbit in the support of $\left(\mathcal{L}^{w}\right)^{o p}$ on $\mathrm{Fl}_{G}$. Its intersection with the preimage of ${ }_{w^{\prime} \cdot \check{\mu}}^{I} \overline{\mathrm{Bun}}_{N^{-}}$under $\overleftarrow{h}_{G}^{\prime}$ can be described as follows.

Note that the pull-back ${ }_{w^{\prime} \cdot \breve{\mu}}^{I} \overline{\operatorname{Bun}}_{N^{-}} \underset{\check{\mu}}{\frac{I}{\operatorname{Bun}_{N^{-}}}}{ }_{\breve{\mu}} \mathcal{N}$ of the $N^{-}[[t]]$-torsor $\check{\mu}_{\breve{N}} \mathcal{V}$ to ${ }_{w^{\prime} \cdot \breve{\mu}}^{I} \overline{\operatorname{Bun}}_{N^{-}}$ admits a reduction to the subgroup $N^{-}[[t]] \cap \operatorname{Ad}_{\left(w^{\prime}\right)^{-1}}(I)$. Then the above intersection identifies with the total space of the bundle associated with the $N^{-}[[t]] \cap \operatorname{Ad}_{\left(w^{\prime}\right)^{-1}}(I)$ space

$$
N^{-}((t)) \cdot(\check{\mu}-\check{\nu}) \cap\left(\operatorname{Ad}_{\left(w^{\prime}\right)^{-1}}(I) \cdot\left(\left(w^{\prime}\right)^{-1} \cdot w(\check{\lambda})\right)\right) \subset N^{-}((t)) \cdot(\check{\mu}-\check{\nu}) \cap \operatorname{Gr}_{G}^{\check{\lambda}} \cdot
$$

Evidently, when $\check{\mu}=\check{\lambda}+\check{\nu}$ and $w^{\prime}=w$ the above intersection is the point-scheme. This means that $\mathrm{IC}_{w \cdot(\check{\lambda}+\check{\nu})}$ indeed appears as a direct summand in the convolution $\mathcal{L}^{w} \star I \mathrm{C}_{\check{\nu}}$. It remains to show that if $\check{\mu} \neq \check{\lambda}+\check{\nu}$ or $w^{\prime} \neq w$, then the scheme in (46) is of dimension strictly less than $\langle\check{\nu}-\check{\mu}+\check{\lambda}, \rho\rangle$.

We will deduce this from Theorem 1.3.5. Let us take $\check{\mu}_{1}$ to be a large dominant coweight and set $\check{\nu}_{1}=\check{\mu}_{1}+\check{\mu}-\check{\nu}$. We will show that if the dimension of (46) violated the above inequality, the perverse sheaf $\mathrm{IC}_{w^{\prime} \cdot \check{\nu}_{1}, \mathrm{Gr}_{G}}$ would appear as a direct summand of $\mathrm{IC}_{w \cdot \check{\lambda}, \mathrm{Gr}_{G}} \star \mathrm{IC}_{\check{\mu}_{1}, \mathrm{Gr}_{G}}$. For that end, it is sufficient to show that the fiber of

$$
(I \cdot(w \cdot \check{\lambda})) \star \operatorname{Gr}_{G}^{\check{\mu}_{1}}
$$

over the point $w^{\prime} \cdot \check{\nu}_{1}$ is of dimension $\geq\langle\check{\nu}-\check{\mu}+\check{\lambda}, \rho\rangle$. We claim that the above fiber contains a subscheme is isomorphic to the scheme (46).

Consider the orbit of the group $\operatorname{Ad}_{w^{\prime}} N^{-}((t))$ passing through $w^{\prime} \cdot \check{\nu}_{1} \in \operatorname{Gr}_{G}$. Its preimage in $(I \cdot(w \cdot \check{\lambda})) \star \operatorname{Gr}_{G}^{\check{\mu}_{1}}$ is the union over parameters $\check{\nu}_{1}^{\prime}$ of the schemes

$$
\left(\left(\operatorname{Ad}_{w^{\prime}} N^{-}((t)) \cdot\left(w^{\prime} \cdot \check{\nu}_{1}^{\prime}\right)\right) \cap(I \cdot(w \cdot \check{\lambda}))\right) \star\left(\left(\operatorname{Ad}_{w^{\prime}} N^{-}((t)) \cdot\left(w^{\prime} \cdot\left(\check{\nu}_{1}-\check{\nu}_{1}^{\prime}\right)\right)\right) \cap \operatorname{Gr}_{G}^{\check{\mu}_{1}}\right),
$$


each of which is fibered over

$$
\left(\operatorname{Ad}_{w^{\prime}} N^{-}((t)) \cdot\left(w^{\prime} \cdot \check{\nu}_{1}^{\prime}\right)\right) \cap(I \cdot(w \cdot \check{\lambda}))
$$

with a typical fiber

$$
\left(\operatorname{Ad}_{w^{\prime}} N^{-}((t)) \cdot\left(w^{\prime} \cdot\left(\check{\nu}_{1}-\check{\nu}_{1}^{\prime}\right)\right)\right) \cap \operatorname{Gr}_{G}^{\check{\mu}_{1}}
$$

Let us take $\check{\nu}_{1}^{\prime}=\check{\nu}_{1}-\check{\mu}_{1}$. We claim that that the intersection of (47) with the preimage of the point $w^{\prime} \cdot \check{\nu}_{1}$ in $(I \cdot(w \cdot \check{\lambda})) \star \operatorname{Gr}_{G}^{\check{\mu}_{1}}$ surjects onto the scheme in (48). This would imply our assertion, since the schemes (46) and (48) are isomorphic for the above choice of $\check{\nu}_{1}^{\prime}$.

This amounts to showing that the subscheme

$$
\left(-\check{\nu}_{1} \cdot\left(w^{\prime}\right)^{-1}\right) \cdot\left(\left(\operatorname{Ad}_{w^{\prime}} N^{-}((t)) \cdot\left(w^{\prime} \cdot(\check{\mu}-\check{\nu})\right)\right) \cap(I \cdot(w \cdot \check{\lambda}))\right)
$$

is contained in $\mathrm{Gr}_{G}^{-w_{0}\left(\check{\mu}_{1}\right)}$.

Let $N^{?}$ be the group-subscheme of $N^{-}((t))$, such that

$$
\left(\operatorname{Ad}_{w^{\prime}} N^{-}((t)) \cdot\left(w^{\prime} \cdot(\check{\mu}-\check{\nu})\right)\right) \cap(I \cdot(w \cdot \check{\lambda}))
$$

is contained in

$$
\left(\operatorname{Ad}_{w^{\prime}}\left(N^{?}\right) \cdot\left(w^{\prime} \cdot(\check{\mu}-\check{\nu})\right)\right) \cap(I \cdot(w \cdot \check{\lambda})) .
$$

We have to show that

$$
\left(-\check{\nu}_{1}\right) \cdot N^{?} \cdot(\check{\mu}-\check{\nu}) \subset \mathrm{Gr}_{G}^{-w_{0}\left(\check{\mu}_{1}\right)}
$$

which is equivalent to

$$
\operatorname{Ad}_{-\check{\nu}_{1}} N^{?} \cdot\left(-\check{\mu}_{1}\right) \subset \operatorname{Gr}_{G}^{-w_{0}\left(\check{\mu}_{1}\right)} .
$$

However, the latter containment is valid, whenever $\check{\nu}_{1}$ is dominant enough so that $\operatorname{Ad}_{-\check{\nu}_{1}}\left(N^{?}\right) \subset N^{-}[[t]]$.

Remark. Let us note that the fiber of $(I \cdot(w \cdot \check{\lambda})) \star \operatorname{Gr}_{G}^{\check{\mu}_{1}}$ over $w^{\prime} \cdot \check{\nu}_{1}$ is in fact entirely contained in the subscheme (47) with $\check{\nu}_{1}^{\prime}=\check{\nu}_{1}-\check{\mu}_{1}$, and it maps to the scheme (48) isomorphically.

To prove the first assertion note that there are only finitely many $\check{\nu}_{1}^{\prime}$ 's, for which the base (48) is non-empty. For any $\check{\nu}_{1}^{\prime}$ other than $\check{\nu}_{1}-\check{\mu}_{1}$ the subscheme

$$
\left(-\check{\nu}_{1} \cdot\left(w^{\prime}\right)^{-1}\right) \cdot\left(\left(\operatorname{Ad}_{w^{\prime}} N^{-}((t)) \cdot\left(w^{\prime} \cdot \check{\nu}_{1}^{\prime}\right)\right) \cap(I \cdot(w \cdot \check{\lambda}))\right)
$$

will have an empty intersection with $\mathrm{Gr}_{G}^{-w_{0}\left(\check{\mu}_{1}\right)}$, because eventually

$$
\left(\operatorname{Ad}_{-\check{\nu}_{1}}\left(N^{?}\right) \cdot\left(\check{\nu}_{1}^{\prime}-\check{\nu}_{1}\right)\right) \cap \operatorname{Gr}_{G}^{-w_{0}\left(\check{\mu}_{1}^{\prime}\right)}=\emptyset .
$$

The second assertion is evident, since every fiber of $\pi: \mathrm{Gr}_{G} \star \mathrm{Gr}_{G} \rightarrow \operatorname{Gr}_{G}$ embeds into the base $\operatorname{Gr}_{G}$. 
MODULES OVER THE SMALL QUANTUM GROUP AND SEMI-INFINITE FLAG MANIFOLD 73

5.3.3. The baby Whittaker case. Our present goal is to prove Theorem 4.4.9. By Verdier duality, it is sufficient to show that the map

$$
\nabla_{\check{\nu}}^{\psi} \rightarrow \mathrm{IC}_{\check{\nu}}^{\psi}
$$

is an isomorphism. Suppose it is not, and let us look at the quotient perverse sheaf; let $\check{\nu}^{\prime}$ be the maximal element of $\check{\Lambda}$, such that this quotient is non-zero when restricted to ${\underset{\nu}{\prime}}^{\prime} \overline{\mathrm{Bun}}_{N^{-}}$. Then this restriction (either $*_{-}$or !-) is a perverse sheaf, and its further restriction onto the locally closed sub-stack of $\frac{1}{\check{\nu}^{\prime}} \operatorname{Bun}_{N^{-}}$equal to $\left(\operatorname{ev}_{\check{\nu}^{\prime}}\right)^{-1}\left(N^{-} \cdot w_{0}\right)$, is a local system.

Hence, we deduce that the Euler characteristic of the ${ }^{*}$-restriction of $\mathrm{IC}_{\check{\nu}}^{\psi}$ to some $\left(\operatorname{ev}_{\check{\nu}^{\prime}}\right)^{-1}\left(N^{-} \cdot w_{0}\right)$ with $\check{\nu}^{\prime} \neq \check{\nu}$ is non-zero. We are going to show that this is impossible by comparing the present situation with the one for $\operatorname{Perv}\left(\mathrm{Gr}_{G}\right)^{I^{-}, \psi}$.

Let us recall that for any $\check{\mu} \in \check{\Lambda}^{+}$, the perverse sheaf $\mathrm{IC}_{\mathrm{Gr}_{G}}^{\psi} \star \mathrm{IC}_{\breve{\mu}, \mathrm{Gr}_{G}}$ is irreducible and is isomorphic to the clean extension of the character sheaf on the $I^{-}$-orbit of the point $w_{0} \cdot\left(\check{\mu}+\check{\rho}^{\prime}\right) \in \mathrm{Gr}_{G}$, by Theorem 2.2.2.

We have the convolution functor

$$
\operatorname{Perv}\left(\operatorname{Gr}_{G}\right)^{I^{-}, \psi} \times \operatorname{Perv}\left(\mathcal{F} l^{\frac{\infty}{2}}\right)^{G[[t]]} \rightarrow \operatorname{Perv}\left(\mathcal{F} l^{\frac{\infty}{2}}\right)^{I^{-}, \psi} .
$$

Theorem 5.3.4. $\mathrm{IC}_{\mathrm{Gr}_{G}}^{\psi} \star \mathrm{I} \mathrm{C}_{\check{\nu}}=\mathrm{IC}_{\check{\nu}+\check{\rho}^{\prime}}^{\psi}$.

We omit the proof, since it essentially repeats the proof of Theorem 5.3.2, where instead of the fact that $\mathcal{L}^{w} \star \mathrm{IC}_{\check{\mu}, \mathrm{Gr}_{G}}$ is irreducible for $\check{\mu} \in \check{\Lambda}^{+}$, we use the above mentioned fact about $\mathrm{IC}_{\mathrm{Gr}_{G}}^{\psi} \star \mathrm{IC}_{\breve{\mu}, \mathrm{Gr}_{G}} \in \operatorname{Perv}\left(\mathrm{Gr}_{G}\right)^{I^{-}, \psi}$.

We claim that the fiber of $\mathrm{IC}_{\mathrm{Gr}_{G}}^{\psi} \star \mathrm{IC}_{\check{\nu}}$ at a point of $\left(\mathrm{ev}_{\check{\nu}^{\prime}}\right)^{-1}\left(N^{-} \cdot w_{0}\right)$ can be written as an extension of certain complexes $\mathcal{K}_{\check{\nu}^{\prime \prime}}$, and the fiber of $\operatorname{IC}_{\mathrm{Gr}_{G}}^{\psi} \star \mathrm{IC}_{\check{\mu}, \mathrm{Gr}_{G}}$ at a point of $I^{-} \cdot\left(w_{0} \cdot\left(\check{\mu}^{\prime}+\check{\rho}^{\prime}\right)\right)$ for $\check{\nu}-\check{\nu}^{\prime}=\check{\mu}-\check{\mu}^{\prime}$ can be written as an exetension of the same complexes.

This would imply our assertion about Euler characteristics, since the fibers of the convolution $\mathrm{IC}_{\mathrm{Gr}_{G}}^{\psi} \star \mathrm{IC}_{\breve{\mu}, \mathrm{Gr}_{G}}$ over $I^{-} \cdot\left(w_{0} \cdot\left(\check{\mu}^{\prime}+\check{\rho}^{\prime}\right)\right)$ are zero unless $\check{\mu}^{\prime}=\check{\mu}$ by cleanness.

For $\check{\nu}^{\prime \prime}$ the complex $K_{\check{\nu}^{\prime \prime}}$ is defined as the fiber of the direct image under

$$
\overleftarrow{h}_{G}^{\prime}:\left(\overleftarrow{h}_{G}^{\prime}\right)^{-1}\left(\stackrel{\check{\nu}}{\prime}_{\check{\operatorname{Bun}}_{N^{-}}}\right) \cap\left(\vec{h}_{G}^{\prime}\right)^{-1}\left(\check{\check{\nu}}^{\prime \prime} \overline{\operatorname{Bun}}_{N^{-}}\right) \cap^{1,0} \mathcal{H}_{G, N^{-}, x}^{\check{\rho}^{\prime}} \rightarrow \underset{\check{\nu}^{\prime}}{k} \overline{\operatorname{Bun}}_{N^{-}}
$$

of the ${ }^{*}$-restriction of $\mathrm{IC}_{\mathrm{Gr}_{G}}^{\psi} \widetilde{\otimes} \mathrm{IC}_{\check{\nu}}$ to the above substack.

Hence, $K_{\check{\nu}^{\prime \prime}}$ is the cohomology with compact supports along the scheme

$$
N((t)) \cdot w_{0} \cdot\left(\check{\nu}^{\prime}-\check{\nu}^{\prime \prime}\right) \cap I^{-} \cdot\left(w_{0} \cdot \check{\rho}^{\prime}\right) \subset \operatorname{Gr}_{G}^{\check{\rho}^{\prime}}
$$

of the complex equal to the tensor product of the character sheaf along $I^{-} \cdot\left(w_{0} \cdot \check{\rho}^{\prime}\right)$ and the constant complex equal to the stalk of $\mathrm{IC}_{\check{\nu}}$ on $\check{\nu}^{\prime \prime} \mathrm{Bun}_{N^{-}}$.

Let us now calculate the fiber of $\operatorname{IC}_{\mathrm{Gr}_{G}}^{\psi} \star \mathrm{IC}_{\check{\mu}, \mathrm{Gr}_{G}}$ at a point of $I^{-} \cdot\left(w_{0} \cdot \check{\mu}^{\prime}\right)$ for $\check{\mu}$ large and $\check{\nu}-\check{\nu}^{\prime}=\check{\mu}-\check{\mu}^{\prime}$. For that we will intersect the fiber of the convolution diagram 
over $w_{0} \cdot \check{\mu}^{\prime}$ with the subschemes of the form

$$
\left(I^{-} \cdot\left(w_{0} \cdot \check{\rho}\right)\right) \star \operatorname{Gr}_{G}^{\check{\mu}^{\prime \prime}} .
$$

As we saw above, each of these intersections is isomorphic to

$$
N((t)) \cdot w_{0} \cdot\left(\check{\mu}^{\prime}-\check{\mu}^{\prime \prime}\right) \cap I^{-} \cdot\left(w_{0} \cdot \check{\rho}\right) \subset \operatorname{Gr}_{G}^{\check{\rho}^{\prime}} .
$$

For each such $\check{\mu}^{\prime \prime}$ the complex that we have to integrate is the tensor product of the character sheaf along $I^{-} \cdot\left(w_{0} \cdot \check{\rho}^{\prime}\right)$ and the stalk of $\mathrm{IC}_{\check{\mu}, \mathrm{Gr}_{G}}$ at $\operatorname{Gr}_{G}^{\check{\mu}^{\prime \prime}}$.

We set up the bijection between $\check{\nu}^{\prime \prime}$ and $\check{\mu}^{\prime \prime}$ so that $\check{\nu}^{\prime \prime}-\check{\nu}^{\prime}=\check{\mu}^{\prime \prime}-\check{\mu}^{\prime}$. Our assertion follows from the fact that for $\check{\lambda}$ small comared with $\check{\mu}$ and $\check{\nu}$ the stalk of $\mathrm{IC}_{\check{\nu}}$ on $\check{\nu}-\check{\lambda} \mathrm{Bun}_{N^{-}}$is isomorphic to the stalk of $\mathrm{IC}_{\check{\mu}, \mathrm{Gr}_{G}}$ on $\mathrm{IC}_{\check{\mu}-\check{\lambda}, \mathrm{Gr}_{G}}$. This follows by combining [FFKM, BFGM] and [Lu, Soe].

\subsection{Action of convolution on standard objects.}

5.4.1. We will now prove the following assertion, parallel to Corollary 3.2.2:

Proposition 5.4.2. If $\check{\lambda}$ is dominant there is a canonical isomorphism

$$
j_{!, \check{\lambda}} \star \nabla_{\check{\nu}} \simeq \nabla_{\check{\nu}+\check{\lambda}}
$$

Proof. Using Proposition 5.1.5, it is sufficient to show that the stalk of $j_{!, \check{\lambda}} \star \nabla_{\check{\nu}}$ is 0 on any ${ }_{\tilde{w}^{\prime}}^{I} \operatorname{Bun}_{N}$ for $\tilde{w}^{\prime} \neq \check{\lambda}+\check{\nu}$, and that it is canonically $\mathbb{C}$ the latter case. This follows in a rather straightforward way from the definition of convolution.

Consider the stack

$$
\left(\overleftarrow{h}_{G}^{\prime}\right)^{-1}\left(\begin{array}{l}
I \\
w^{\prime} \cdot \check{\nu}^{\prime}
\end{array} \overline{\operatorname{Bun}}_{N^{-}}\right) \cap\left(\vec{h}_{G}^{\prime}\right)^{-1}\left({ }_{w \cdot \check{\nu}}^{I} \overline{\operatorname{Bun}}_{N^{-}}\right) \cap{ }^{I, I} \mathcal{H}_{G, N^{-}, x}^{\check{\lambda}}
$$

projecting to ${ }_{w^{\prime} \cdot \check{\nu}^{\prime}}^{I} \overline{\mathrm{Bun}}_{N^{-}}$by means of $\overleftarrow{h}_{G}^{\prime}$. In the above formula $\mathcal{H}_{G, N, x}^{\check{\lambda}}$ is the locally closed substack of $I, I \mathcal{H}_{G, N^{-}, x}^{\check{\lambda}}$, corresponding to the $I$-orbit $I \cdot \check{\lambda} \subset \mathrm{Fl}_{G}$.

The fiber of the above stack over a point of ${ }_{w^{\prime} \cdot \check{\nu}^{\prime}}^{I} \overline{\mathrm{Bun}}_{N^{-}}$is isomorphic to

$$
\left(N^{-}((t)) \cdot\left(\check{\nu}^{\prime}-\check{\nu}\right) \cdot w^{-1}\right) \cap\left(\left(w^{\prime}\right)^{-1} \cdot I \cdot \check{\lambda}\right) \subset \mathrm{Fl}_{G} .
$$

Set $w=1$, and we claim that the above intersection is empty unless $\check{\nu}^{\prime}=\check{\nu}+\check{\lambda}$ and $w^{\prime}=1$, and that in the latter case, this is a point-scheme.

The latter assertion is evident. To prove the first one, we will use the following:

Lemma 5.4.3. For $\check{\lambda}$ dominant,

$$
N^{-}((t)) \cdot B[[t]] \supset \operatorname{Ad}_{\check{\lambda}}(I) \subset B[[t]] \cdot N^{-}((t)) .
$$

Using the lemma, it is enough to show that

$$
\left(w^{\prime} \cdot\left(\check{\nu}^{\prime}-\check{\nu}\right) \cdot N^{-}((t))\right) \cap\left(N^{+}((t)) \cdot \check{\lambda}\right) \subset G((t))
$$

is non-empty only if $w^{\prime}=1$ and $\check{\nu}^{\prime}-\check{\nu}=\check{\lambda}$, which is evident from the Bruhat decomposition. 
MODULES OVER THE SMALL QUANTUM GROUP AND SEMI-INFINITE FLAG MANIFOLD 75

5.4.4. Let us now exhibit a compatibility relation between the isomorphisms of Proposition 5.4.2 and Proposition 5.2.4. Namely, we claim that for $\check{\lambda} \in \check{\Lambda}^{+}$the diagrams

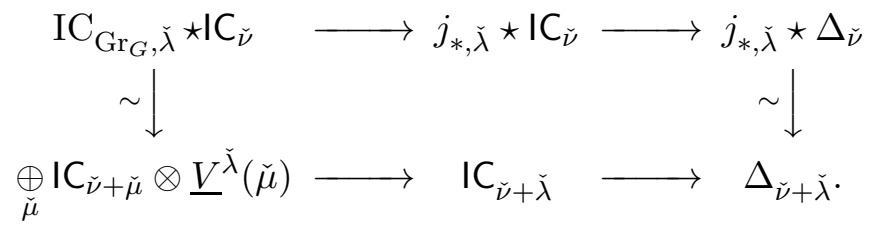

and

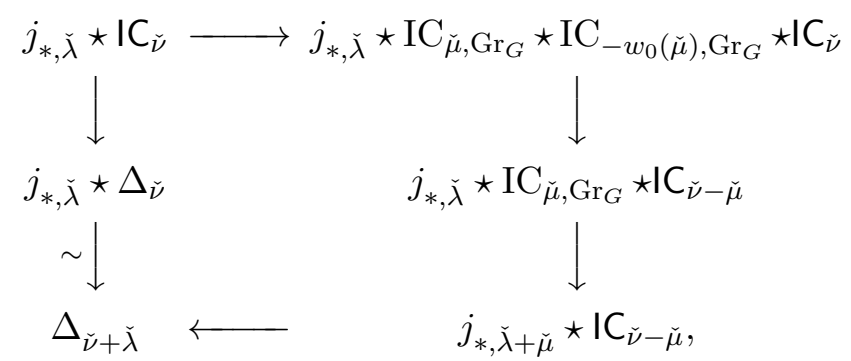

are commutative. This follows from the definition of the isomorphisms in both cases.

Note that Proposition 5.4.2 implies that for $\check{\nu}$ dominant

$$
j_{!,-\check{\nu}} \star \nabla_{w_{0} \cdot \check{\nu}^{\prime}} \simeq \nabla_{w_{0} \cdot\left(\check{\nu}^{\prime}-w_{0}(\check{\nu})\right)},
$$

and hence

$$
j_{*, \check{\nu}} \star \nabla_{w_{0} \cdot \check{\nu}^{\prime}} \simeq \nabla_{w_{0} \cdot\left(\check{\nu}^{\prime}+w_{0}(\check{\nu})\right)} .
$$

Consider now the morphism

$$
\mathrm{IC}_{\check{\nu}} \rightarrow \nabla_{w_{0} \cdot(\check{\nu}+2 \check{\rho})},
$$

obtained by Verdier duality from Sect. 4.4.15. By construction, the space of such morphisms for every $\check{\nu}$ is a 1-dimensional vector space, canonically independent of $\check{\nu}$. From the construction one infers the following:

Lemma 5.4.5. For $\check{\nu} \in \check{\Lambda}, \check{\lambda}, \check{\mu} \in \check{\Lambda}^{+}$the diagrams

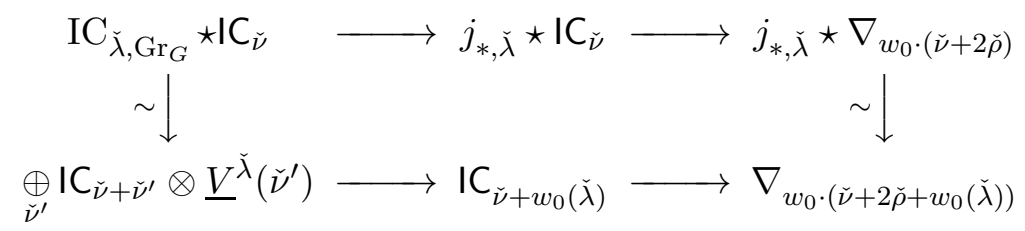

and

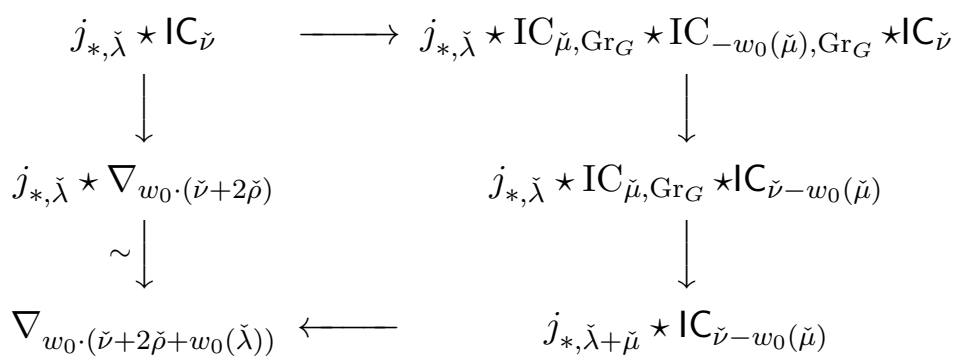

are commutative. 


\section{The Equivalence}

\subsection{The functor.}

6.1.1. Let $\dot{\mathcal{S}}$ be an object of $\dot{\mathrm{H} e c k e}\left(\mathrm{Gr}_{G}, \check{G}\right)^{G^{k}}$. We attach to it a covariant functor on $\overline{\operatorname{Perv}}\left(\mathcal{F} l^{\frac{\infty}{2}}\right)^{G^{k}}$ as follows. To an object $\mathcal{F} \in \overline{\operatorname{Perv}}\left(\mathcal{F} l^{\frac{\infty}{2}}\right)^{G^{k}}$ we assign the set of collections of morphisms $\dot{\mathcal{S}}_{\check{\lambda}} \star \mathrm{I} \mathrm{C}_{\check{\lambda}} \rightarrow \mathcal{F}$, such that for any $V \in \operatorname{Rep}(\check{G})$ and $\check{\mu} \in \check{\Lambda}$, the diagram

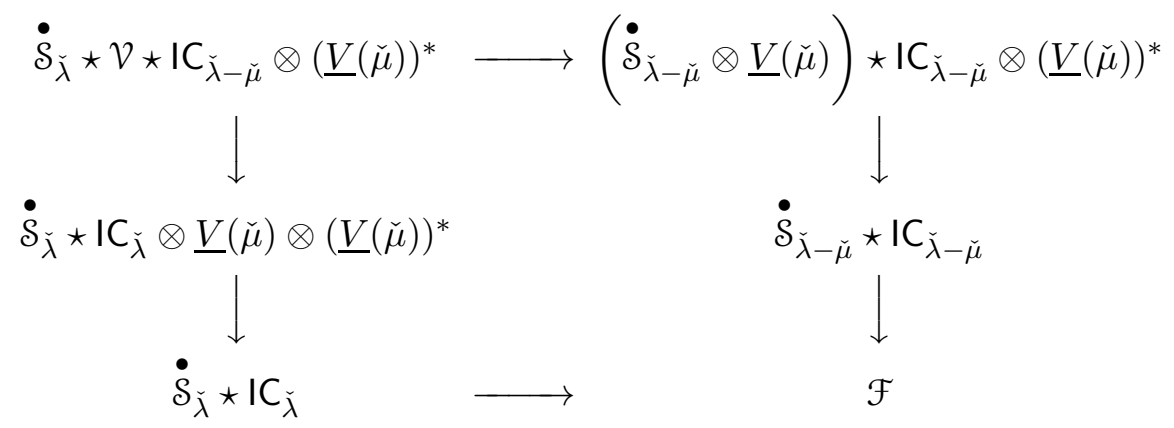

commutes, where the upper horizontal arrow is given by the Hecke morphism for $\dot{\mathcal{S}}$, and the left vertical arrow by Proposition 5.2.4.

It is easy to see that the above functor is representable by

$$
\operatorname{co-eq}\left(\underset{\check{\lambda}, \check{\mu}, V}{\oplus} \dot{\mathcal{S}}_{\check{\lambda}} \star \mathcal{V} \star \mathrm{I} C_{\check{\lambda}-\check{\mu}} \otimes(\underline{V}(\check{\mu}))^{*} \rightrightarrows \underset{\check{\nu}}{\oplus} \dot{\mathcal{S}}_{\check{\nu}} \star \mathrm{IC} C_{\check{\nu}}\right)
$$

where the two arrows correspond to the two circuits of the above commutative diagram.

We denote the resulting functor $\dot{\operatorname{Hecke}}\left(\mathrm{Gr}_{G}, \check{G}\right)^{G^{k}} \rightarrow \overline{\operatorname{Perv}}\left(\mathcal{F} l^{\frac{\infty}{2}}\right)^{G^{k}}$ by Conv ${ }^{\text {Hecke. By }}$ construction, Conv ${ }^{\text {Hecke }}$ is right-exact.

Proposition 6.1.2. For $\stackrel{\mathcal{S}}{=}=\mathcal{S} \star \dot{\mathcal{R}}_{\breve{G}}\{\check{\mu}\} \in \dot{H}_{\text {ecke }}\left(\mathrm{Gr}_{G}, \check{G}\right)^{G^{k}}$ the object Conv ${ }^{\operatorname{Hecke}}(\dot{\mathcal{S}})$ is canonically isomorphic to $\mathcal{S} \star \mathrm{IC}-\check{\mu}$.

Proof. For a morphism Conv ${ }^{\text {Hecke }}(\dot{\mathcal{S}}) \rightarrow \mathcal{F}$, by taking its component $\dot{\mathcal{S}}_{-\check{\mu}} \star \mathrm{IC}_{-\check{\mu}} \rightarrow \mathcal{F}$ we obtain a map $\mathcal{S} \star \mathrm{IC}_{-\check{\mu}} \rightarrow \mathcal{F}$, since

$$
\dot{\mathcal{S}}_{-\check{\mu}} \simeq \mathcal{S} \star \dot{\mathcal{R}}_{\breve{G}}\{\check{\mu}\}_{-\check{\mu}} \simeq \mathcal{S} \star\left(\dot{\mathcal{R}}_{\breve{G}}\right)_{0}
$$

and it contains $\mathcal{S}$ as a direct summand.

Vice versa, having a map $\mathcal{S} \star \mathbb{I C}_{-\check{\mu}} \rightarrow \mathcal{F}$, for every $V \in \operatorname{Rep}(\check{G})$ and $\check{\lambda}$ we define a map

by

$$
\left(\mathcal{S} \star \mathcal{V} \otimes \underline{V}^{*}(\check{\lambda}+\check{\mu})\right) \star \mathrm{IC} \check{\lambda}_{\check{\lambda}} \rightarrow \mathcal{F}
$$

$$
\left(\mathcal{S} \star \mathcal{V} \otimes \underline{V}^{*}(\check{\lambda}+\check{\mu})\right) \star \mathrm{I} C_{\check{\lambda}} \rightarrow \mathcal{S} \star \mathrm{IC}-\check{\mu} \otimes \underline{V}(-\check{\lambda}-\check{\mu}) \otimes \underline{V}^{*}(\check{\lambda}+\check{\mu}) \rightarrow \mathcal{S} \star \mathrm{IC}-\check{\mu} \rightarrow \mathcal{F} .
$$

The fact that the resulting system of maps satisfies the defining condition follows from the second assertion in Proposition 5.2.4. 
We also have the following assertion that follows from Proposition 5.2.4(2):

Lemma 6.1.3. For $\dot{\mathcal{S}} \in \dot{\mathrm{H} e c k e}\left(\mathrm{Gr}_{G}, \check{G}\right)^{G^{k}}$ and $\check{\mu} \in \check{\Lambda}$,

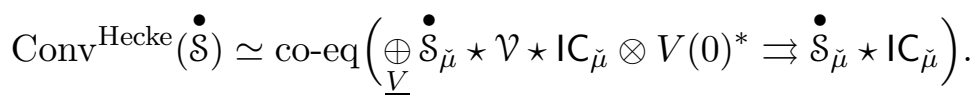

6.1.4. We propose the following:

Conjecture 6.1.5. The functor

$$
\text { Conv }{ }^{\text {Hecke }}: \dot{\operatorname{Hecke}}\left(\mathrm{Gr}_{G}, \check{G}\right)^{G^{k}} \rightarrow \overline{\operatorname{Perv}}\left(\mathcal{F} l^{\frac{\infty}{2}}\right)^{G^{k}}
$$

is exact and fully-faithful.

In fact, we think that Conv ${ }^{\text {Hecke }}$ is very close to be an equivalence of categories. Unfortunately, we cannot formulate a precise conjecture, due to our lack of understanding of Noetherian properties of both categories. In any case, we think that one can express $\operatorname{Perv}\left(\mathcal{F} l^{\frac{\infty}{2}}\right)^{G^{k}}$ completely in terms of $\dot{\operatorname{Hecke}}\left(\mathrm{Gr}_{G}, \check{G}\right)^{G^{k}}$, which would then supply a local (in particular, independent of the global curve $X$ ) description of $\operatorname{Perv}\left(\mathcal{F l} l^{\frac{\infty}{2}}\right)^{G^{k}}$.

In what follows we are going to discuss a version of the above conjecture, where instead of the level $G^{k}$ we take $I^{0}$. In this case it would be possible to formulate and prove a more precise result.

Theorem 6.1.6. The functor

$$
\text { Conv }{ }^{\text {Hecke }}: \dot{\text { Hecke }}\left(\mathrm{Gr}_{G}, \check{G}\right)^{I^{0}} \rightarrow \overline{\operatorname{Perv}}\left(\mathcal{F} l^{\frac{\infty}{2}}\right)^{I^{0}}
$$

is exact, and it defines an equivalence between the sub-categories of Artinian objects on both sides.

Since the subcategory $\stackrel{\dot{H}}{\mathrm{e} e c k e}\left(\mathrm{Gr}_{G}, \check{G}\right)_{A r t}^{I^{0}}$ of Artinian objects in $\dot{H}_{\text {ecke }}\left(\mathrm{Gr}_{G}, \check{G}\right)^{I^{0}}$ is equivalent to $\dot{\mathfrak{u}}_{\ell}-\bmod _{0}$, as a corollary we obtain:

Theorem 6.1.7. The category $\dot{\mathfrak{u}}_{\ell}-\bmod _{0}$ is equivalent to the category of Artinian objects in $\operatorname{Perv}\left(\mathcal{F} l^{\frac{\infty}{2}}\right)^{I^{0}}$.

6.1.8. Here we would like to add the following observation.

As we saw above, the category $\operatorname{Hecke}\left(\mathrm{Gr}_{G}, \check{G}\right)^{I^{0}}$ is acted on by the group $W_{\text {aff }}$ by self-equivalences: the elements of $\check{\Lambda}$ act by shifting the grading, and $w \in W$ by the twisting functors $\dot{\mathcal{S}} \mapsto w \dot{\mathcal{S}}$ (which on the level of $\dot{\mathfrak{u}}_{\ell}$-mod correspond to the functors $\left.\mathrm{F}_{w}\right)$. Evidently, these functors preserve the subcategory $\dot{H}$ ecke $\left(\operatorname{Gr}_{G}, \check{G}\right)_{\text {Art }}^{I^{0}}$, and, hence, the carry over to the category of Artinian objects in $\operatorname{Perv}\left(\mathcal{F} l^{\frac{\infty}{2}}\right)^{I^{0}}$.

Let us describe how these functors act on the irreducibles of $\operatorname{Perv}\left(\mathcal{F} l^{\frac{\infty}{2}}\right)^{I^{0}}$. For $w \in W$ let $\mathcal{L}^{w}=\mathrm{IC}_{w \cdot \check{\lambda}, \operatorname{Gr}_{G}}$ be the corresponding "restricted" irreducible in $\operatorname{Perv}\left(\operatorname{Gr}_{G}\right)^{I}$. By Theorem 5.3.2 and Proposition 6.1.2,

$$
\operatorname{Conv}^{\text {Hecke }}\left(\mathcal{L}^{w} \star \dot{\mathcal{R}}_{\breve{G}}\{\check{\mu}\}\right) \simeq \mathrm{IC}_{w \cdot(\check{\lambda}-\check{\mu})} .
$$


Hence,

$$
\left(\mathrm{IC} \mathrm{C}_{w \cdot \check{\nu}}\right)\{\check{\mu}\} \simeq \mathrm{IC} \mathrm{w}_{w \cdot(\check{\nu}-\check{\mu})}
$$

and

$$
\mathrm{F}_{w^{\prime}}\left(\mathrm{IC}_{w \cdot(\check{\lambda}-\check{\mu})}\right) \simeq \mathrm{IC}_{w \cdot\left(\check{\lambda}-w^{\prime}(\check{\mu})\right)}
$$

Recall that the $\mathbb{C}$-linearized Grothendieck group of the category of Artinian objects in $\operatorname{Perv}\left(\mathcal{F} l^{\frac{\infty}{2}}\right)^{0}$ identifies with Lusztig's periodic module over the affine Hecke algebra (cf. $[\mathrm{FFKM}])^{9}$, and hence, also with the space of Iwahori-invariant functions in the Schwarz space of [BK]. Equation (55) implies that the maps on the Grothendieck group, induced by the functors $\mathrm{F}_{w}$, are equal to the Fourier transform operators, introduced in [BK].

The rest of the paper is devoted to the proof of Theorem 6.1.6.

\subsection{Proof of the equivalence.}

6.2.1. As a first step we prove the following:

Proposition 6.2.2. The functor

$$
\text { Conv }{ }^{\text {Hecke }}: \dot{\text { Hecke }}\left(\mathrm{Gr}_{G}, \check{G}\right)^{I^{0}} \rightarrow \overline{\operatorname{Perv}}\left(\mathcal{F} l^{\frac{\infty}{2}}\right)^{I^{0}}
$$

is exact.

The present subsection is devoted to the proof of this proposition.

Since $\dot{H}$ ecke $\left(\mathrm{Gr}_{G}, \check{G}\right)^{I^{0}}$ is the ind-completion of the subcategory of its Artinian objects, it is sufficient to prove that Conv ${ }^{\text {Hecke }}$ restricted to $\dot{H}_{\text {ecke }}\left(\operatorname{Gr}_{G}, \check{G}\right)_{A r t}^{I^{0}}$ is exact.

Let

$$
0 \rightarrow \dot{\mathcal{S}}_{1} \rightarrow \dot{\mathcal{S}}_{2} \rightarrow \dot{\mathcal{S}} \rightarrow 0
$$

be a short exact sequence of objects of $\dot{H}$ ecke $\left(\mathrm{Gr}_{G}, \check{G}\right)_{A r t}^{I^{0}}$. We have to show that Conv $^{\text {Hecke }}\left(\dot{\mathcal{S}}_{1}\right) \rightarrow$ Conv $^{\text {Hecke }}\left(\dot{\mathcal{S}}_{2}\right)$ is injective. For that we may assume that $\dot{\mathcal{S}}$ is simple. By Sect. 1.3.8, $\dot{\mathcal{S}}$ is then isomorphic to $\mathcal{S} \star \dot{\mathcal{R}}_{\breve{G}}\{\check{\mu}\}$ for $\mathcal{S} \in \operatorname{Perv}\left(\operatorname{Gr}_{G}\right)^{I^{0}}$.

We can find an object $\mathcal{S}^{\prime} \in \operatorname{Perv}\left(\mathrm{Gr}_{G}\right)^{I^{0}}$ with a surjection $\mathcal{S}^{\prime} \rightarrow \mathcal{S}$, and a map $\mathcal{S}^{\prime} \rightarrow\left(\dot{\mathcal{S}}_{2}\right)_{-\check{\mu}}$ in $\overline{\operatorname{Perv}}\left(\mathrm{Gr}_{G}\right)^{I^{0}}$, such that the diagram

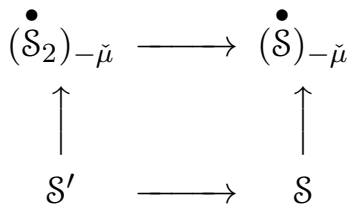

\footnotetext{
${ }^{9}$ For this to be formally true we have to pass to the category of mixed D-modules of Hodge-Tate type in $\operatorname{Perv}\left(\mathcal{F} l^{\frac{\infty}{2}}\right)^{I^{0}}$
} 

is commutative. Hence, we obtain a map $\mathcal{S}^{\prime} \star \dot{\mathcal{R}}_{\breve{G}}\{\check{\mu}\} \rightarrow \dot{\mathcal{S}}_{2}$. Let $\dot{\mathcal{S}}_{2}^{\prime}$ be the Cartesian product of $\dot{\mathcal{S}}_{2}$ and $\mathcal{S}^{\prime} \star \dot{\mathcal{R}}_{\check{G}}\{\check{\mu}\}$ over $\mathcal{S} \star \dot{\mathcal{R}}_{\check{G}}\{\check{\mu}\}$. We have a commutative diagram:

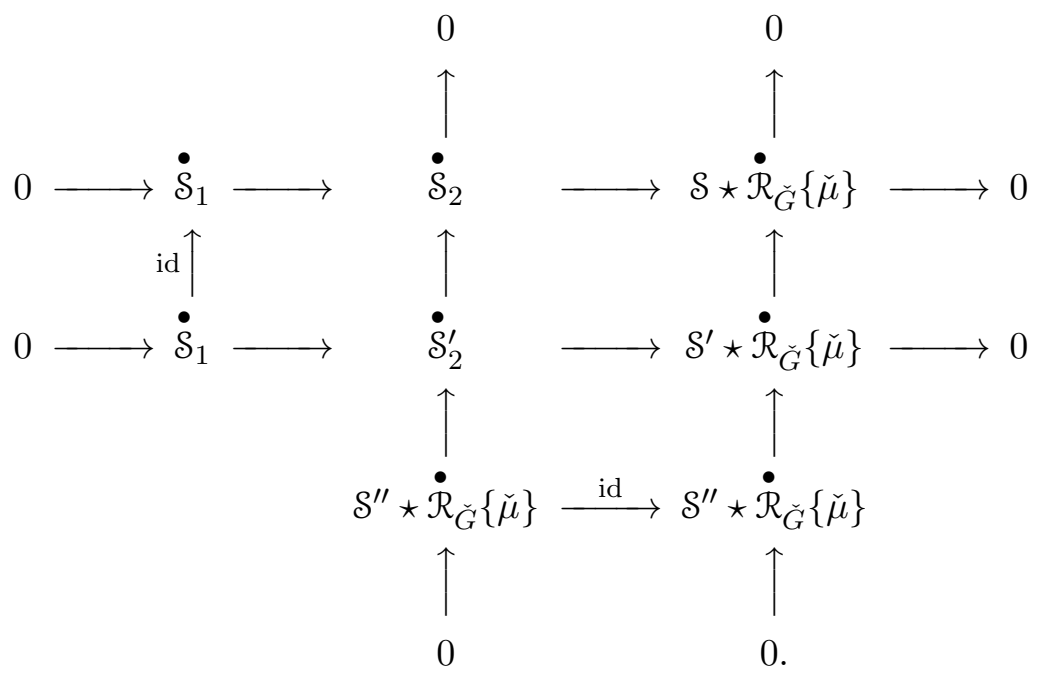

It is enough to show that the map

$$
\mathrm{Conv}^{\text {Hecke }}\left(\dot{\mathcal{S}}_{1} \oplus \mathcal{S}^{\prime \prime} \star \dot{\mathcal{R}}_{\breve{G}}\{\check{\mu}\}\right) \rightarrow \text { Conv }^{\text {Hecke }}\left(\dot{\mathcal{S}}_{2}^{\prime}\right)
$$

is injective. However, by construction, $\dot{\mathcal{S}}_{2}^{\prime}$ splits as a direct sum $\dot{\mathcal{S}}_{1} \oplus \mathcal{S}^{\prime} \star \dot{\mathcal{R}}_{\breve{G}}\{\check{\mu}\}$. Hence, it is enough to show that the map

$$
\operatorname{Conv}^{\text {Hecke }}\left(\mathcal{S}^{\prime \prime} \star \dot{\mathcal{R}}_{\check{G}}\{\check{\mu}\}\right) \rightarrow \operatorname{Conv}^{\text {Hecke }}\left(\mathcal{S}^{\prime} \star \dot{\mathcal{R}}_{\check{G}}\{\check{\mu}\}\right)
$$

is injective. But the latter results from Proposition 6.1.2 combined with Theorem 5.2.2, since the map in question comes from a map $\mathcal{S}^{\prime \prime} \rightarrow \mathcal{S}^{\prime}$ in $\operatorname{Perv}\left(\operatorname{Gr}_{G}\right)^{I^{0}}$.

6.2.3. Recall that the Verdier duality functor $\mathbb{D}$ is defined on $\dot{H}$ ecke $\left(\operatorname{Gr}_{G}, \check{G}\right)_{A r t}^{I^{0}}$. In this subsection we will prove the following:

Proposition 6.2.4. The functor $\mathrm{Conv}^{\mathrm{Hecke}}$ commutes with the Verdier duality.

Recall that if $\dot{\mathcal{S}} \in \dot{\operatorname{Hecke}}\left(\mathrm{Gr}_{G}, \check{G}\right)_{A r t}^{I^{0}}$ is an object represented as

$$
\operatorname{coker}\left(\mathcal{S}_{1} \star \dot{\mathcal{R}}_{\check{G}}\left\{\check{\mu}_{1}\right\} \rightarrow \mathcal{S}_{2} \star \dot{\mathcal{R}}_{\breve{G}}\left\{\check{\mu}_{2}\right\}\right)
$$

then $\mathbb{D}(\dot{\mathcal{S}})$ is described as follows:

The map $\mathcal{S}_{1} \star \dot{\mathcal{R}}_{\breve{G}}\left\{\check{\mu}_{1}\right\} \rightarrow \mathcal{S}_{2} \star \dot{\mathcal{R}}_{\breve{G}}\left\{\check{\mu}_{2}\right\}$ comes from a map $\alpha: \mathcal{S}_{1} \rightarrow \mathcal{S}_{2} \star \mathcal{V} \otimes \underline{V}^{*}\left(\check{\mu}_{2}-\check{\mu}_{1}\right)$ defined for some $V \in \operatorname{Rep}(\breve{G})$. By adjunction, we have a map

$$
\mathcal{S}_{1} \star \mathbb{D}\left(\mathcal{V}^{o p}\right) \otimes \underline{V}\left(\check{\mu}_{1}-\check{\mu}_{2}\right) \rightarrow \mathcal{S}_{2},
$$

and applying the Verdier duality we obtain a map

$$
\mathbb{D}(\alpha): \mathbb{D}\left(\mathcal{S}_{2}\right) \rightarrow \mathbb{D}\left(\mathcal{S}_{1}\right) \star \mathcal{V}^{o p} \otimes \underline{V}^{*}\left(\check{\mu}_{2}-\check{\mu}_{1}\right) .
$$


Recall that the functor $\mathcal{V} \mapsto \mathbb{D}\left(\mathcal{V}^{o p}\right)$ corresponds on the level of $\operatorname{Rep}(\check{G})$ to the dualization functor $V \mapsto V^{*}$, whereas $\mathcal{V} \mapsto \mathbb{D}(\mathcal{V})$ corresponds to the contragredient duality $V \mapsto V^{\vee}$. In particular, $\underline{V}(\check{\mu}) \simeq \underline{V}^{o p}(-\check{\mu})$.

We then obtain a morphism

$$
\mathbb{D}\left(\mathcal{S}_{2}\right) \star \dot{\mathcal{R}}_{\breve{G}}\left\{\check{\mu}_{2}\right\} \rightarrow \mathbb{D}\left(\mathcal{S}_{1}\right) \star \dot{\mathcal{R}}_{\breve{G}}\left\{\check{\mu}_{1}\right\}
$$

whose kernel ia $\mathbb{D}(\dot{\mathcal{S}})$.

For $\dot{\mathcal{S}}$ as above, by Proposition $6.1 .2, \operatorname{Conv}^{\operatorname{Hecke}}(\dot{\mathcal{S}}) \simeq \operatorname{coker}(\beta)$, where $\beta$ is the map

$$
\begin{aligned}
& \mathcal{S}_{1} \star I C_{-\check{\mu}_{1}} \rightarrow\left(\mathcal{S}_{2} \star \mathcal{V} \star I C_{-\check{\mu}_{1}}\right) \otimes \underline{V}^{*}\left(\check{\mu}_{2}-\check{\mu}_{1}\right) \rightarrow \\
& \mathcal{S}_{2} \star I C_{-\check{\mu}_{2}} \otimes \underline{V}\left(\check{\mu}_{1}-\check{\mu}_{2}\right) \otimes \underline{V}^{*}\left(\check{\mu}_{2}-\check{\mu}_{1}\right) \rightarrow \mathcal{S}_{2} \star \mathrm{IC}-\check{\mu}_{2} .
\end{aligned}
$$

By Proposition 6.2.2, Conv $^{\text {Hecke }}(\mathbb{D}(\dot{\mathcal{S}})) \simeq \operatorname{ker}(\gamma)$, where $\gamma$ is the map

$$
\begin{aligned}
& \mathbb{D}\left(\mathcal{S}_{2}\right) \star \mathrm{IC}-\check{\mu}_{2} \rightarrow\left(\mathbb{D}\left(\mathcal{S}_{1}\right) \star \mathcal{V}^{o p} \star \mathrm{IC}-\check{\mu}_{2}\right) \otimes \underline{V}^{*}\left(\check{\mu}_{2}-\check{\mu}_{1}\right) \rightarrow \\
& \mathbb{D}\left(\mathcal{S}_{1}\right) \star \mathrm{IC} \check{\check{\mu}}_{1} \otimes \underline{V}^{o p}\left(\check{\mu}_{2}-\check{\mu}_{1}\right) \otimes \underline{V}^{*}\left(\check{\mu}_{2}-\check{\mu}_{1}\right) \rightarrow \mathbb{D}\left(\mathcal{S}_{1}\right) \star \mathrm{IC}-\check{\mu}_{1} .
\end{aligned}
$$

To prove the proposition it remains to see that the morphisms $\beta$ and $\gamma$ are transformed into one-another by Verdier duaility. This is evident when $V$ is the trivial representation. By transitivity, this reduces the assertion to the case when $\mathcal{S}_{1} \simeq$ $\mathcal{S}_{2} \star \mathcal{V} \otimes \underline{V}^{*}\left(\check{\mu}_{2}-\check{\mu}_{1}\right)$.

In the latter case, both arrows $\mathbb{D}(\beta)$ and $\gamma$ are obtained from the corresponding arrows for $\mathcal{S}_{2}$ replaced by $\delta_{1, \mathrm{Gr}_{G}}$ by convolution with $\mathcal{S}_{2}$. The case $\mathcal{S}_{2}=\delta_{1, \mathrm{Gr}_{G}}$ is a straightforward verification.

6.2.5. We will now state a crucial result, from which we will deduce Theorem 6.1.6.

Theorem 6.2.6. For $w \in W$ and $\check{\mu} \in \check{\Lambda}$,

$$
\mathrm{Conv}^{\text {Hecke }}\left(\dot{\mathcal{M}}^{w \cdot \check{\mu}}\right) \simeq \Delta_{w \cdot \check{\mu}} \text {. }
$$

We will now deduce Theorem 6.1.6 from Theorem 6.2.6. Consider now the following general set-up:

Let $\mathcal{C}$ be an abelian Artinian category; let $A$ be the set parametrizing its irreducibles; for $a \in A$ we will denote by $\mathcal{L}^{a}$ the corresponding object. Assume also that for each $a \in A$ there exist objects $\nabla^{a}$ and $\Delta^{a}$, such that $\mathcal{L}^{a}$ is the cosocle of $\nabla^{a}$ and the socle of $\Delta^{a}$. Assume, moreover, that $\operatorname{Ext} t^{i}\left(\nabla^{a^{\prime}}, \Delta^{a^{\prime \prime}}\right)=0$ for $i=1,2$, and $\operatorname{Hom}\left(\nabla^{a^{\prime}}, \Delta^{a^{\prime \prime}}\right)=0$ unless $a^{\prime}=a^{\prime \prime}$, and in the latter case it is 1-dimensional (which implies that any element in $\operatorname{Hom}\left(\nabla^{1}, \Delta^{a}\right)$ factors through $\left.\mathcal{L}^{a}\right)$.

Let now $\mathcal{C}_{1}$ and $\mathcal{C}_{2}$ be two such categories with the same set of irreducibles $A$. Let $\mathrm{G}: \mathcal{C}_{1} \rightarrow \mathcal{C}_{2}$ be an exact functor, such that $\mathrm{G}\left(\mathcal{L}_{1}^{a}\right) \simeq \mathcal{L}_{2}^{a}, \mathrm{G}\left(\nabla_{1}^{a}\right) \simeq \nabla_{2}^{a}, \mathrm{G}\left(\Delta_{1}^{a}\right) \simeq \Delta_{2}^{a}$.

Lemma 6.2.7. Under the above circumstances, $\mathrm{G}$ is an equivalence of categories.

Theorem 6.1.6 follows from this lemma, using Corollary 4.4.7, Lemma 3.2.19, Proposition 6.2.2, (54), Proposition 6.2.6 and Proposition 6.2.4. 
MODULES OVER THE SMALL QUANTUM GROUP AND SEMI-INFINITE FLAG MANIFOLD 81

6.2.8. Proof of Lemma 6.2.7. Note first of all that the assumption implies that G is faithful.

Step 1. For $a, a^{\prime} \in A$ consider the long exact sequences

$$
\begin{aligned}
& 0 \rightarrow \operatorname{Hom}\left(\mathcal{L}_{i}^{a}, \Delta_{i}^{a^{\prime}}\right) \rightarrow \operatorname{Hom}\left(\nabla_{i}^{a}, \Delta_{i}^{a^{\prime}}\right) \rightarrow \operatorname{Hom}\left(\operatorname{ker}\left(\nabla_{i}^{a} \rightarrow \mathcal{L}_{i}^{a}\right), \Delta_{i}^{a^{\prime}}\right) \rightarrow \\
& \operatorname{Ext}^{1}\left(\mathcal{L}_{i}^{a}, \Delta_{i}^{a^{\prime}}\right) \rightarrow \operatorname{Ext}\left(\nabla_{i}^{a}, \Delta_{i}^{a^{\prime}}\right)=0
\end{aligned}
$$

for $i=1,2$. Since $\operatorname{Hom}\left(\nabla_{1}^{a}, \Delta_{1}^{a^{\prime}}\right) \rightarrow \operatorname{Hom}\left(\nabla_{2}^{a}, \Delta_{2}^{a^{\prime}}\right)$ is an isomorphism, comparing the two, we infer that $\operatorname{Ext}^{1}\left(\mathcal{L}_{1}^{a}, \Delta_{1}^{a^{\prime}}\right) \rightarrow \operatorname{Ext}^{1}\left(\mathcal{L}_{2}^{a}, \Delta_{2}^{a^{\prime}}\right)$ is injective.

Step 2. Consider now the long exact sequence

$$
\begin{aligned}
& 0 \rightarrow \operatorname{Hom}\left(\mathcal{L}_{1}^{a}, \mathcal{L}_{1}^{a^{\prime}}\right) \rightarrow \operatorname{Hom}\left(\mathcal{L}_{1}^{a}, \Delta_{1}^{a^{\prime}}\right) \rightarrow \operatorname{Hom}\left(\mathcal{L}_{1}^{a}, \Delta_{1}^{a^{\prime}} / \mathcal{L}_{1}^{a^{\prime}}\right) \rightarrow \\
& \operatorname{Ext}^{1}\left(\mathcal{L}_{1}^{a}, \mathcal{L}_{1}^{a^{\prime}}\right) \rightarrow \operatorname{Ext}{ }^{1}\left(\mathcal{L}_{1}^{a}, \Delta_{1}^{a^{\prime}}\right) \rightarrow \operatorname{Ext} t^{1}\left(\mathcal{L}_{1}^{a}, \Delta_{1}^{a^{\prime}} / \mathcal{L}_{1}^{a^{\prime}}\right)
\end{aligned}
$$

for $i=1,2$. $\operatorname{Hom}\left(\mathcal{L}_{1}^{a}, \Delta_{1}^{a^{\prime}}\right) \rightarrow \operatorname{Hom}\left(\mathcal{L}_{2}^{a}, \Delta_{2}^{a^{\prime}}\right)$ is an isomorphism and using Step 1, we find that $\operatorname{Ext}^{1}\left(\mathcal{L}_{1}^{a}, \mathcal{L}_{1}^{a^{\prime}}\right) \rightarrow \operatorname{Ext}^{1}\left(\mathcal{L}_{2}^{a}, \mathcal{L}_{2}^{a^{\prime}}\right)$ is injective.

Step 3. Let $\mathcal{F}^{\prime}$ be any object of $\mathcal{C}_{1}$. Using Step 3 , by induction on the length of $\mathcal{F}^{\prime}$, we find that the map $\operatorname{Hom}\left(\mathcal{L}_{1}^{a}, \mathcal{F}^{\prime}\right) \rightarrow \operatorname{Hom}\left(\mathcal{L}_{2}^{a}, \mathrm{G}\left(\mathcal{F}^{\prime}\right)\right)$ is an isomorphism.

Step 4. Returning to the long exact sequence of Step 1, we find that the map $\operatorname{Ext}^{1}\left(\mathcal{L}_{1}^{a}, \Delta_{1}^{a^{\prime}}\right) \rightarrow \operatorname{Ext}^{1}\left(\mathcal{L}_{2}^{a}, \Delta_{2}^{a^{\prime}}\right)$ is an isomorphism.

Step 5. Again, by induction on the length, using Step 3, we show that the map $\operatorname{Ext}^{1}\left(\mathcal{L}_{1}^{a}, \mathcal{F}^{\prime}\right) \rightarrow \operatorname{Ext}^{1}\left(\mathcal{L}_{2}^{a}, \mathrm{G}\left(\mathcal{F}^{\prime}\right)\right)$ is injective.

Step 6. By the exact sequence of Step 2, from Step 4 we find that $\operatorname{Ext}^{1}\left(\mathcal{L}_{1}^{a}, \mathcal{L}_{1}^{a^{\prime}}\right) \rightarrow$ $\operatorname{Ext}^{1}\left(\mathcal{L}_{2}^{a}, \mathcal{L}_{2}^{a^{\prime}}\right)$ is an isomorphism.

Step 7. Let $\mathcal{F}$ be an object of $\mathcal{C}_{1}$, and $\mathcal{F}^{\prime}$ some other object. By induction on the length of $\mathcal{F}$, from Step 5 we obtain that $\operatorname{Hom}\left(\mathcal{F}, \mathcal{F}^{\prime}\right) \rightarrow \operatorname{Hom}\left(\mathrm{G}(\mathcal{F}), \mathrm{G}\left(\mathcal{F}^{\prime}\right)\right)$ is an isomorphism.

Hence, $G$ is fully-faithful. To finish the proof of the lemma, we have to show that $G$ induces isomorphsims on the level of $\operatorname{Ext}^{1}(\cdot, \cdot)$.

Step 8. By induction on the length of $\mathcal{F}$, from Step 5 and Step 7 we obtain that $\operatorname{Ext}^{1}\left(\mathcal{F}, \mathcal{F}^{\prime}\right) \rightarrow \operatorname{Ext}^{1}\left(\mathrm{G}(\mathcal{F}), \mathrm{G}\left(\mathcal{F}^{\prime}\right)\right)$ is injective.

Step 9. For $a, a^{\prime} \in A$ consider the long exact sequences

$$
\begin{aligned}
& \ldots 0=\operatorname{Ext}^{1}\left(\nabla_{i}^{a}, \Delta_{i}^{a^{\prime}}\right) \rightarrow \operatorname{Ext}^{1}\left(\operatorname{ker}\left(\nabla_{i}^{a} \rightarrow \mathcal{L}_{i}^{a}\right), \Delta_{i}^{a^{\prime}}\right) \rightarrow \\
& \operatorname{Ext}^{2}\left(\mathcal{L}_{i}^{a}, \Delta_{i}^{a^{\prime}}\right) \rightarrow \operatorname{Ext}^{2}\left(\nabla_{i}^{a}, \Delta_{i}^{a^{\prime}}\right)=0
\end{aligned}
$$

for $i=1,2$. From Step 8 we infer that $\operatorname{Ext}^{2}\left(\mathcal{L}_{1}^{a}, \Delta_{1}^{a^{\prime}}\right) \rightarrow \operatorname{Ext}\left(\mathcal{L}_{2}^{a}, \Delta_{2}^{a^{\prime}}\right)$ is injective.

Step 10. Consider the long exact sequence

$$
\begin{aligned}
& \operatorname{Ext}^{1}\left(\mathcal{L}_{1}^{a}, \mathcal{L}_{1}^{a^{\prime}}\right) \rightarrow \operatorname{Ext}^{1}\left(\mathcal{L}_{1}^{a}, \Delta_{1}^{a^{\prime}}\right) \rightarrow \operatorname{Ext}^{1}\left(\mathcal{L}_{1}^{a}, \Delta_{1}^{a^{\prime}} / \mathcal{L}_{1}^{a^{\prime}}\right) \rightarrow \\
& \operatorname{Ext}^{2}\left(\mathcal{L}_{1}^{a}, \mathcal{L}_{1}^{a^{\prime}}\right) \rightarrow \operatorname{Ext} t^{2}\left(\mathcal{L}_{1}^{a}, \Delta_{1}^{a^{\prime}}\right) \rightarrow \operatorname{Ext}^{2}\left(\mathcal{L}_{1}^{a}, \Delta_{1}^{a^{\prime}} / \mathcal{L}_{1}^{a^{\prime}}\right)
\end{aligned}
$$

By Step 4, Step 8 and Step 9, the map $\operatorname{Ext}\left(\mathcal{L}_{1}^{a}, \mathcal{L}_{1}^{a^{\prime}}\right) \rightarrow \operatorname{Ext}\left(\mathcal{L}_{2}^{a}, \mathcal{L}_{2}^{a^{\prime}}\right)$ is injective.

Step 11. By induction on length, from Step 6, we obtain that $\operatorname{Ext}^{1}\left(\mathcal{L}_{1}^{a}, \mathcal{F}^{\prime}\right) \rightarrow$ $\operatorname{Ext}^{1}\left(\mathcal{L}_{2}^{a}, \mathrm{G}\left(\mathcal{F}^{\prime}\right)\right)$ is an isomorphism. 
Step 12. Again, by induction on the length, from Step 10 and Step 6, we obtain that the map $\operatorname{Ext}^{2}\left(\mathcal{L}_{1}^{a}, \mathcal{F}^{\prime}\right) \rightarrow \operatorname{Ext}^{2}\left(\mathcal{L}_{2}^{a}, \mathrm{G}\left(\mathcal{F}^{\prime}\right)\right)$ is injective.

Step 13. Finally, by induction on the length of $\mathcal{F}$, from Steps 11 and 12 we infer that the map $\operatorname{Ext}^{1}\left(\mathcal{F}, \mathcal{F}^{\prime}\right) \rightarrow \operatorname{Ext}^{1}\left(\mathrm{G}(\mathcal{F}), \mathrm{G}\left(\mathcal{F}^{\prime}\right)\right)$ is an isomorphism.

\subsection{Identification of the image of baby co-Verma modules.}

6.3.1. In this subsection we will prove Theorem 6.2.6. Note that it suffices to show that

$$
\operatorname{Conv}^{\text {Hecke }}\left(\dot{\mathcal{M}}^{1}\right) \simeq \Delta_{0},
$$

since all other isomorphisms will then hold by (16), (3.2.2) and (5.4.2).

We construct a map

$$
\operatorname{Conv}{ }^{\text {Hecke }}\left(\dot{\mathcal{M}}^{1}\right) \rightarrow \Delta_{0}
$$

as follows. We need to construct the maps

$$
\underset{\check{\lambda}}{\lim } j_{*, \check{\lambda}+\check{\mu}} \star \mathrm{IC}_{-w_{0}(\check{\lambda}), \operatorname{Gr}_{G}} \star \mathrm{IC}-\check{\mu} \rightarrow \Delta_{0}
$$

for every $\check{\mu}$.

For $\check{\lambda} \in \check{\Lambda}^{+}$as above we have a map

$$
\begin{aligned}
& j_{*, \check{\lambda}+\check{\mu}} \star \mathrm{IC}_{-w_{0}(\check{\lambda}), \operatorname{Gr}_{G}} \star \mathrm{IC}-\check{\mu} \simeq \underset{\check{\nu}}{\oplus} j_{*, \check{\lambda}+\check{\mu}} \star \mathrm{IC} C_{-\check{\mu}+\check{\nu}} \otimes \underline{V}^{*}(\check{\nu}) \rightarrow j_{*, \check{\lambda}+\check{\mu}} \star \mathrm{IC} C_{-\check{\mu}-\check{\lambda}} \rightarrow \\
& j_{*, \check{\lambda}+\check{\mu}} \star \Delta_{-\check{\mu}-\check{\lambda}} \simeq \Delta_{0},
\end{aligned}
$$

The fact that these maps are compatible with the maps in the inductive system that defines $\dot{\mathcal{M}}^{1}$, follows from the commutativity of the diagrams (50) and (51). The fact that the resulting system of maps

$$
\dot{\mathcal{M}}_{\check{\mu}}^{1} \star \mathrm{IC}_{\check{\mu}} \rightarrow \Delta_{0}
$$

factors through Conv ${ }^{\text {Hecke }}\left(\dot{\mathcal{M}}^{1}\right)$ follows from (45).

6.3.2. Now, we claim that the map Conv ${ }^{\text {Hecke }}\left(\dot{\mathcal{M}}^{1}\right) \rightarrow \Delta_{0}$ constructed above is nonzero in the quotient category ${ }^{f} \operatorname{Perv}\left(\mathcal{F} l^{\frac{\infty}{2}}\right)^{I^{0}}$. Using Proposition 4.4.12, it is enough to show that the map

$$
\operatorname{Av}_{N^{-}, \psi}\left(\operatorname{Conv}^{\text {Hecke }}\left(\dot{\mathcal{M}}^{1}\right)\right) \rightarrow \operatorname{Av}_{N^{-}, \psi}\left(\Delta_{0}\right)
$$

is non-zero. The latter reduces to showing that for $\check{\lambda}$ dominant and regular, the map

$$
j_{*, \check{\lambda}} \star \mathrm{IC}_{\check{\nu}} \rightarrow \Delta_{\check{\lambda}+\check{\nu}}
$$

gives rise to a non-zero map

$$
\operatorname{Av}_{I^{-}, \psi}\left(\mathcal{W}^{*, \check{\lambda}}\right) \star \mathrm{IC}_{\check{\nu}} \rightarrow \operatorname{Av}_{N^{-}, \psi}\left(\Delta_{\check{\lambda}+\check{\nu}}\right)
$$

However, the latter is straightforward from the definition of convolution. 
MODULES OVER THE SMALL QUANTUM GROUP AND SEMI-INFINITE FLAG MANIFOLD 83

In particular, by Corollary 4.4.13(2), we obtain that the map of (56) is surjective. Moreover, it is an isomorphism in the quotient category ${ }^{f} \operatorname{Perv}\left(\mathcal{F} l^{\frac{\infty}{2}}\right)^{I^{0}}$ by $\operatorname{Proposi-}$ tion 3.2.6(1).

We claim that in order to finish the proof of the theorem, it suffices to show that there exists a non-zero map

$$
\Delta_{0} \rightarrow \operatorname{Conv}^{\text {Hecke }}\left(\dot{\mathcal{M}}^{1}\right) \text {. }
$$

Indeed, if such a map exists, its image in ${ }^{f} \operatorname{Perv}\left(\mathcal{F} l^{\frac{\infty}{2}}\right)^{I^{0}}$ cannot be 0 by Corollary 4.4.13, and hence the composition

$$
\Delta_{0} \rightarrow \operatorname{Conv}^{\text {Hecke }}\left(\dot{\mathcal{M}}^{1}\right) \rightarrow \Delta_{0}
$$

is non-zero. Then the above composition is the identity map on $\Delta_{0}$, up to a scalar.

Hence, it would remain to show that Conv ${ }^{\text {Hecke }}\left(\dot{\mathcal{M}}^{1}\right)$ is indecomposable. We claim that it in fact does not admit irreducible quotients besides the canonical map

$$
\mathrm{Conv}^{\text {Hecke }}\left(\dot{\mathcal{M}}^{1}\right) \rightarrow \mathrm{Conv}^{\text {Hecke }}\left(\mathcal{L}^{w_{0}} \star \dot{\mathcal{R}}_{\check{G}}\left\{\check{\rho}^{\prime}\right\}\right) \text {. }
$$

This is so because Conv ${ }^{\text {Hecke }}\left(\dot{\mathcal{M}}^{1}\right)$ cannot map to any partially integrable irreducible object of $\operatorname{Perv}\left(\mathcal{F} l^{\frac{\infty}{2}}\right)^{I^{0}}$ by the same argument as in the proof of Proposition 2.3.2, and by Corollary 3.2.6(1), Conv ${ }^{\text {Hecke }}\left(\mathcal{L}^{w_{0}} \star \dot{\mathcal{R}}_{\breve{G}}\left\{\check{\rho}^{\prime}\right\}\right)$ is the only non-partially integrable constituent of Conv ${ }^{\text {Hecke }}\left(\dot{\mathcal{M}}^{1}\right)$.

6.3.3. Thus, our goal is to construct a map as in (57). By Proposition 3.2.10 and Proposition 6.2.4, it suffices to construct a map

$$
\mathrm{Conv}^{\text {Hecke }}\left(\left({ }^{w_{0}} \dot{\mathcal{M}}^{w_{0}}\right)\{2 \check{\rho}\}\right) \rightarrow \nabla_{0},
$$

or, equivalently, a map

$$
\mathrm{Conv}^{\text {Hecke }}\left(\left({ }^{w_{0}} \dot{\mathcal{M}}^{1}\right)\{2 \check{\rho}\}\right) \rightarrow \nabla_{w_{0}}
$$

Consider the inductive system that defines $\left(\left({ }^{w_{0}} \dot{\mathcal{M}}^{1}\right)\{2 \check{\rho}\}\right) \check{\mu}$, viewed as an object of $\overline{\operatorname{Perv}}\left(\operatorname{Gr}_{G}\right)^{I^{0}}$ :

$$
\underset{\check{\lambda}}{\lim _{\longrightarrow}} j_{*, \check{\lambda}+\check{\rho}^{\prime}-w_{0}(\check{\mu})+2 \check{\rho}} \star \mathrm{IC}_{-w_{0}(\check{\lambda}), \mathrm{Gr}_{G}} .
$$

For every such $\check{\mu}$ and $\check{\lambda}$, we define the map

$$
j_{*, \check{\lambda}-w_{0}(\check{\mu})+2 \check{\rho}} \star \mathrm{IC}_{-w_{0}(\check{\lambda}), \mathrm{Gr}_{G}} \star \mathrm{IC}_{\check{\mu}} \rightarrow \nabla_{w_{0}}
$$

as the composition:

$$
\begin{aligned}
& j_{*, \check{\lambda}-w_{0}(\check{\mu})+2 \check{\rho}} \star \mathrm{IC}_{-w_{0}(\check{\lambda}), \mathrm{Gr}_{G}} \star \mathrm{I} \mathrm{C}_{\check{\mu}} \simeq \bigoplus_{\check{\nu}} j_{*, \check{\lambda}+2 \check{\rho}-w_{0}(\check{\mu})} \star \mathrm{I} \mathrm{C}_{\breve{\mu}+\check{\nu}} \otimes\left(\underline{V}^{\check{\lambda}}\right)^{*}(\check{\nu}) \rightarrow \\
& \rightarrow j_{*, \check{\lambda}+2 \check{\rho}-w_{0}(\check{\mu})} \star \mathrm{IC}_{\check{\mu}-w_{0}(\check{\lambda})} \rightarrow j_{*, \check{\lambda}+2 \check{\rho}-w_{0}(\check{\mu})} \star \nabla_{w_{0} \cdot\left(\check{\mu}-w_{0}(\check{\lambda})+2 \check{\rho}\right)} \simeq \nabla_{w_{0}},
\end{aligned}
$$

where the third arrow comes from (53), and the last arrow comes from (52). 
The fact that these maps for various $\check{\lambda}$ are compatible with the maps in the inductive system follows from Lemma 5.4.5. The fact that the resulting map

$$
\operatorname{Conv}\left(\left({ }^{w_{0}} \dot{\mathcal{M}}^{1}\right)\{2 \check{\rho}\}\right) \rightarrow \nabla_{w_{0}}
$$

factors through Conv ${ }^{\text {Hecke }}\left(\left(w_{0} \dot{\mathcal{M}}^{1}\right)\{2 \check{\rho}\}\right) \rightarrow \nabla_{w_{0}}$ follows from (45).

\section{REFERENCES}

[ABG] S. Arkhipov, R. Bezrukavnikov, V. Ginzburg, Quantum groups, the loop Grassmannian, and the Springer resolution, J. Amer. Math. Soc. 17 (2004), 595-678.

[AB] S. Arkhipov, R. Bezrukavnikov, Perverse sheaves on affine flags and Langlands dual group, math.RT/0201073.

[AG] S.Arkhipov, D. Gaitsgory, Another realization of the category of modules over the small quantum group, Adv. in Math 173 (2003), 114-143.

[BBM] R. Bezrukavnikov, A. Braverman, I. Mirković, Some results about geometric Whittaker model, Adv. Math. 186 (2004) 143-152.

[BG] A. Braverman, D. Gaitsgory, Geometric Eisenstein series, Inv. Math. 150 (2002), 287-384.

[BG1] A. Braverman, D. Gaitsgory, Crystals via the affine Grassmannian, Duke Math. J. 107 (2001), $561-575$.

[BFGM] A. Braverman, M. Finkelberg, D. Gaitsgory, I. Mirkovic, Intersection cohomology of Drinfeld's compactifications, Selecta Math. (N.S.) 8 (2002), 381-418.

[BK] A. Braverman, D. Kazhdan, On the Schwartz space of the basic affine space, Selecta Math. (N.S.) 5 (1999), 1-28.

[FFKM] B Feigin, M. Finkelberg, A. Kuznetsov, I. Mirkovic, Semi-infinite flags. II. Local and global intersection cohomology of quasimaps' spaces. Differential topology, infinite-dimensional Lie algebras, and applications, 113-148, Amer. Math. Soc. Transl. Ser. 2, 194, Amer. Math. Soc., Providence, RI, 1999.

[FF] B. Feigin, E. Frenkel, Affine Kac-Moody algebras and semi-infinite flag manifolds, Comm. Math. Phys. 128 (1990) 161-189.

[FM] M. Finkelberg, I. Mirkovic, Semi-infinite flags. I. Case of global curve $\mathbf{P}^{1}$. Differential topology, infinite-dimensional Lie algebras, and applications, 81-112, Amer. Math. Soc. Transl. Ser. 2, 194, Amer. Math. Soc., Providence, RI, 1999.

[FG] E. Frenkel, D. Gaitsgory, Tamely ramified local Langlands correspondence for affine Kac-Moody algebras, forthcoming.

[FGV] E. Frenkel, D. Gaitsgory, K. Vilonen, Whittaker patterns in the geometry of moduli spaces of bundles on curves, Ann. Math. 153 (2001), 699-748.

[KL] D. Kazhdan, G. Lusztig, Tensor structures arising from affine Lie algebras. III, IV, J. Amer. Math. Soc. 7 (1994), 383-453, 335-381.

[KT] M. Kashiwara, T. Tanisaki, The Kazhdan-Lusztig conjecture for affine algebras with negative level, Duke Math. J. 77 (1995), 383-453.

[Lu] G. Lusztig, Singularities, character formulas, and a q-analog of weight multiplicities, Analysis and topology on singular spaces, II, III (Luminy, 1981), 208-229, Astrisque 101-102.

[Lu1] G. Lusztig, Introduction to quantum groups, Progress in Mathematics, 110. Birkhuser Boston, Inc., Boston, MA, 1993.

[MV] I. Mirkovic, K. Vilonen, Geometric Langlands duality and representations of algebraic groups over commutative rings, math.RT/0401222.

[Soe] W. Soergel, Character formulas for tilting modules over Kac-Moody algebras, Rep. Theory 2 (1998), 432-448 
E-mail address: serguei.arkhipov@yale.edu, bezrukav@math.northwestern.edu, braval@math.brown.edu, gaitsgde@math.harvard.edu, mirkovic@math.umass.edu 